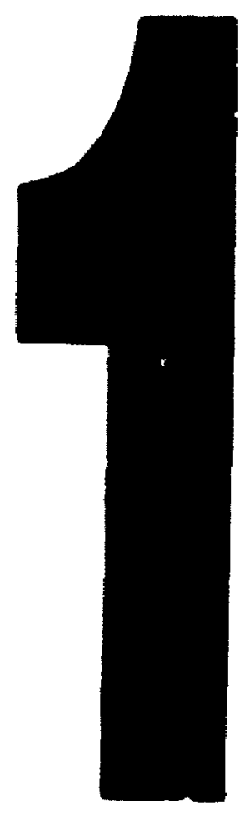

PM-1 31/2"x4" PHOTOCRAPHIC MICROCOPY TARCET wes 10 tas ANSI/180 "2 EOUIVALENT

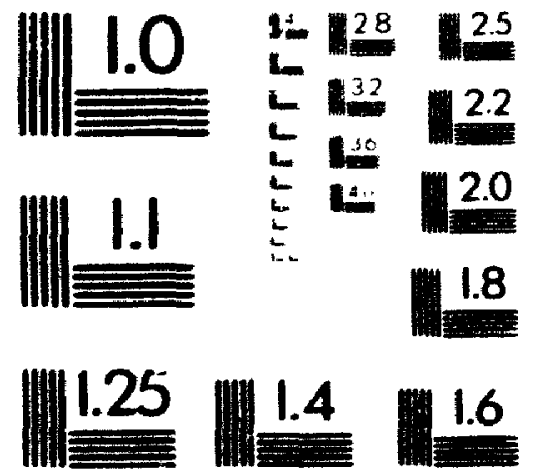

PAECISHON"W RESOUUTION TARGETS 


\section{Mixed Analog-Digital Design Considerations in Deep Submicron CMOS Technologies}

By

Seyfi Bazarjani

A thesis submitted to the Faculty of Graduate Studies and Research in partial fulfillment of the requirements for the degree of Doctor of Philosophy.

Ottawa-Carleton Institute for Electrical Engineering Department of Electronics

Faculty of Engineering

Carleton University

Ottawa, Canada

24 July 1996

() Seyfi S. Bazarjani 1996 
Acquisitions and

Bibliographic Services Branch

395 Wetlington Street

Onawa Onfark

KIA ONA du Canada

Oirection des acqunsitions et

des services bibliographiques

395 tue Welington

Ottawa (Ontain)

KIA ONA row itse votre ieverence

Our the Notereterence
The author has granted an irrevocable non-exclusive licence allowing the National Library of Canada to reproduce, loan, distribute or sell copies of his/her thesis by any means and in any form or format, making this thesis available to interested persons.
L'auteur a accordé une licence irrévocable et non axclusive permettant à la Bibliotheqque nationale du Canada de reproduire, prêter, distribuer ou vendre des copies de sa thèse de quelque manière et sous quelque forme que ce soit pour mettre des exemplaires de cette thèse à la disposition des personnes intéressées.

L'auteur conserve la propriété du droit d'auteur qui protège sa thèse. Ni la thèse ni des extraits substantiels de celle-ci ne doivent être imprimés ou autrement reproduits sans son autorisation.

ISBN 0-612-19354-3 
Nom steyfollah Bazarjani

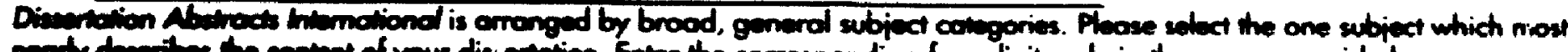
neaty decertbes the comient of your distentotion. Enver the corresponding four digit code in the spoces provided.

$$
\text { Elestronics and Elez-Ticul Enjinceing }
$$

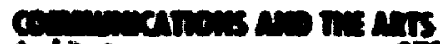

Artiming

0728

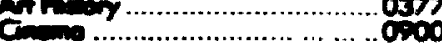

Com

Pot

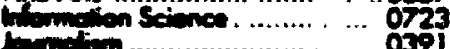

trman

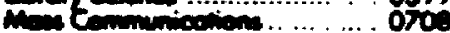

Mnic . .................. 0413

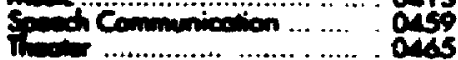

\section{Empris:}

Genet ...............................

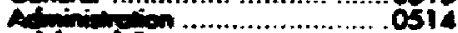

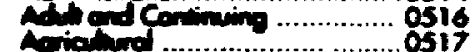

Arialind ............................ O517

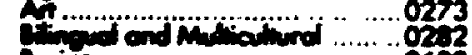

mence... 060

Commen Cot.............. o275

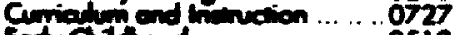

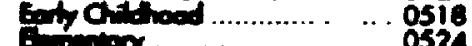

Fon . ...................... O524

Gridenowicouneding. osis

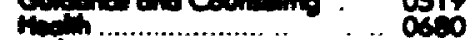

Fin

Hon

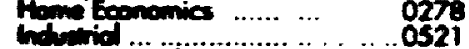

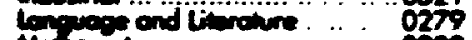

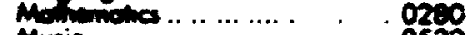

Mric .....................

Prriced... ............... 0523

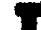

\section{minerestion}

Apriatione

Comard ................. 0473

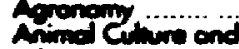

0285

Mution ................... , 0475

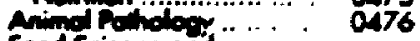

Food scimcend

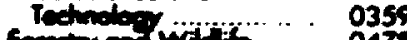

fondy on widis. . .047

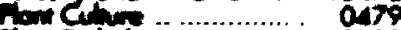

mont R.hedowy ................ O480

fromt pryiology ............... g817

Wood Tedricogy. ....... .. 074

solo.

Gyed .................. . 0306

Anow. ..............

Godutivion ....... ... .. 030.

Con ................ .

Ecoloer............................ 032

Envondory ......... . .... 0353

Contio ....................

tindeg ............ . . ..079

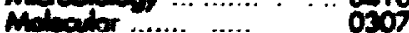

Nomecionce.................. . .317

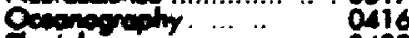

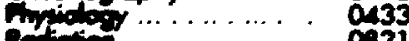

entivion. ... .082

Yominary science .. . .... 0778

sieftyic

Nutis

0786

grineang

Ciesochninimy

0425
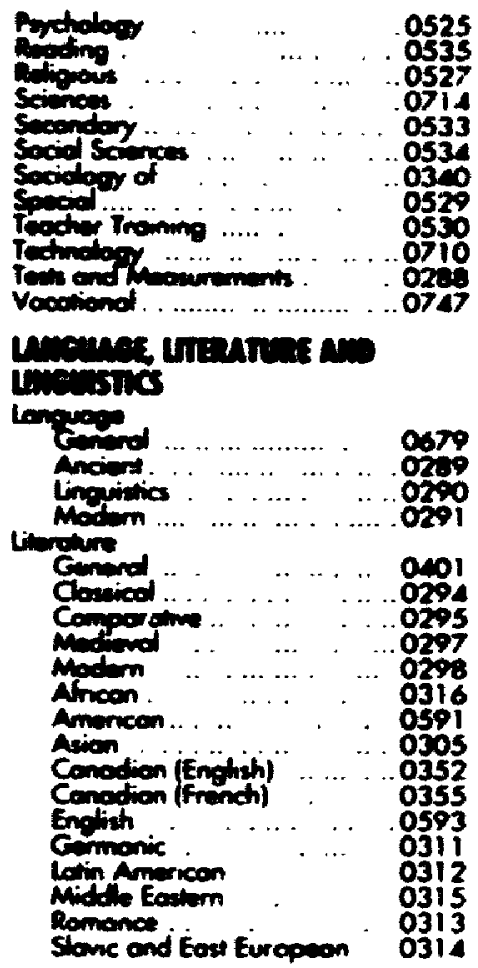

\section{Pmaserit, encess ans}

Iincolos

Philowoph

Religon

Citierd

Cores Studves

Mierey of

Pitorophy of

Thadey

segurstans

Animeon sudes

Antropologr

Anchoologr

Culined

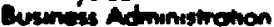

Genend

Accountim

Conting

Monicemen

Conadion Suides

Economics

Goriciturol

Commarce- busuness

Finonces

Anstory

Laber

follthery

Goorophy

Geromology

Histor

Gemeral

stum cost

0422

C318

0321

0319

0322

0469

0323

0324

C327

0310

0272

045

0330

0385

osol

0503

$0 \operatorname{sos}$

asio

OSII

0358

0578

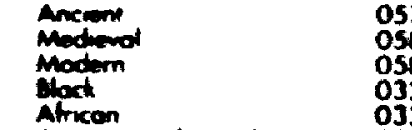

0581

0328

Ano, Aviroho and Oceoma 0332

Conodian 0334

Furopen 0335

tomn Amuricon 0330

mando Eoum $\quad 0333$

Uhind Senen

Hution of science

Palincel Serince

Genered

mernowond low and

Rulowion

Puble Adonmustinton

0308

Recrition

Socal Wor

Secrobogy

Crmmortaty and Puncloory

Demoreaph

Ether and Rocol Studes

indruduad ond fombly

Studen

Indusemol and labo

Relomions

Puble and Sociol Wellore

Social Sorvenum and

Dendopoment

Theory and Memods

Tromportivion

Utbon and Reguanol Plannung

Women is Sudres

0615

0.616

0617

osis

0626

0827

0631

0628

0620

0,330

0700

0344

0700

0953

\section{Puerunanuse}

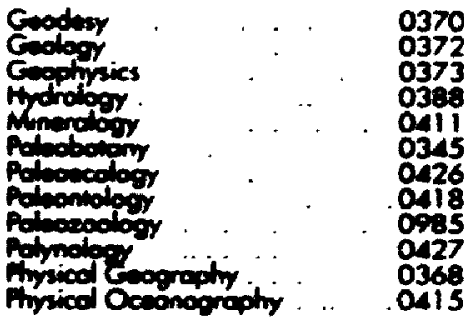

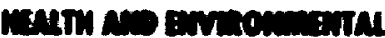

Stistis

Emingonnemel Sciences

.0768

todith scinent

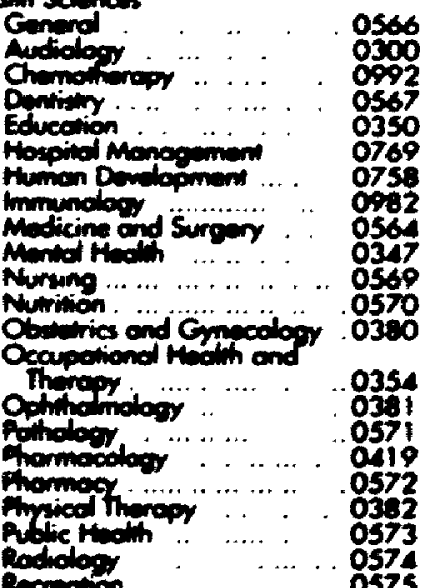

Speech Pothology

Hoxicology

mirsen sainas

Pure Sciences

Chemisiny

Generol

Arroculuro

Bochomishy

increganc

Mucloor

Organk

Aromocented

Plynucel

Poymer

Motriemencs

Promes:

Generd

Acovines:

Astronomy and

Antrophrucs:

Amospheric science

Aromix

Electroncs and Electicty

Elomertory porndes and

Hint Eropy

Fund and floma

Moleculor

Nudion

Opeses

Radigion

surivicics

Anilied Saimes:

Aplied mechonucs

Compun serence
0.60

0386

0485

0749

0486

ouse

0738

$04 \%$

ouss

0754

oess

0605

0

0,008

0408

0607

0798

0759

0610

0752

0750

OS1:

0.463

0346

0904

Engunewing

Aeroupoce

Agrecuther

Acomotim

somedice.

Chemicol

Crval

Electronics and Electrical

Hoot ond thermodymomins

Hydroule

Industrol

Marme

Movereats Serence

Mechomicol

Matholurg

Mening

Pactoging

Percioum

concinar

Sysiem scance

Goolectrnolog

Opwotnom Revorch

Trosics Technolog/

Texdle Tectmoloor

estamesy

Conerel 
The undersigned recommend to the Faculty of Graduate Studies and Research the acceptance of the thesis

"Mixed Analog-Digital Design Considerations in Deep Submicron CMOS Technologies" submitted by Seyfollah S. Bazarjani in partial fulfillment of the requirements for the degree of Doctor of Philosophy.
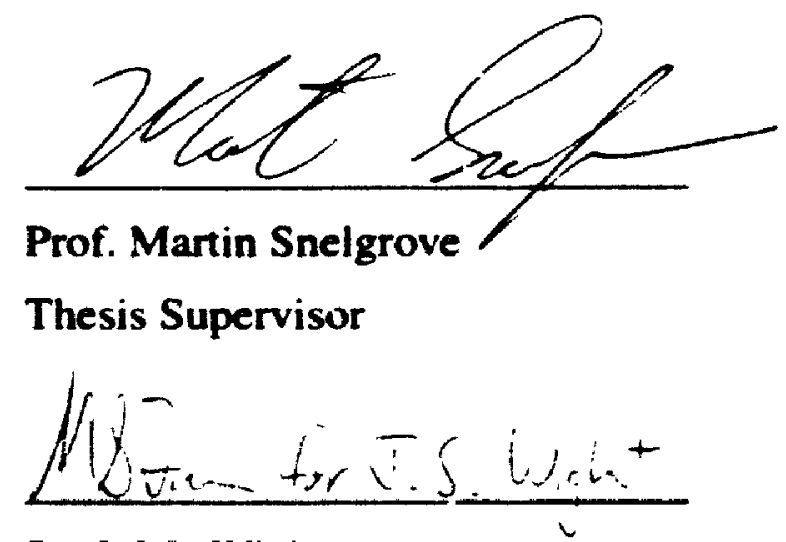

Prof. J.S. Wight

Chairman,

Department of Electronics

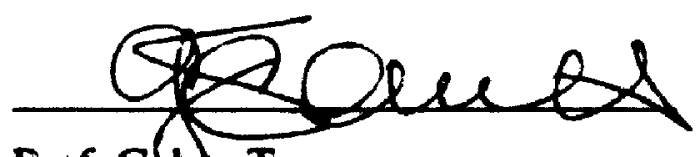

Prof. Gabor Temes

External Examiner

Ontawa-Carleton Institute for Electrical Engineering

Department of Electronics

Faculty of Engineering

Carleton University

24 July 1996 


\section{Abstract}

Higher speed and higher density are the main thrusts of CMOS technology and are achieved by device miniaturization. In deep submicron geometries, the supply voltage is scaled down to prevent reliability hazards such as oxide breakdown and hot carrier effects and also to reduce energy per operation of digital circuits. Lowering the supply voltage directly reduces the signal swing. which in turn makes the design of high-speed wide dynamic range mixed-signal SC circuits a challenge. It is also desirable to implement SC circuits in a standard digital CMOS process, where double-poly capacitors are not available. because the driving forces beinind the CMOS technologies are DRAMs and microprocessors which do not require linear capacitors.

This thesis demonstrates that analog circuit design can track projected digital technology until at least the year 2010. It addresses the design of low-voltage and high-speed SC circuits and also explores the feasibility of using MOSFET capacitors in a linear SC circuit. The thesis describes an experimental $1 \mathrm{~V}$ CMOS process implemented as a subset of a $0.5 \mu \mathrm{m}$ dual $\mathrm{n}^{+} / \mathrm{p}^{+}$poly gate process using natural MOSFETs. A $1 \mathrm{~V}$ experimental SC sigma-delta modulator is presented-the lowest supply voltage reported so far for SC $\Sigma \Delta$ modulators. Also described is a high-speed fourth-order SC bandpass $\Sigma \Delta$ inodulator and a novel fourth-order double-sampled SC bandpass $\Sigma \Delta$ modulator together with their experimental results. Finally, a fourth-order bandpass SC $\Sigma \Delta$ modulator designed in a digital CMOS process using pMOSFET capacitors is described. 


\section{Acknowledgments}

I wish to expre's my gratitude to my advisor Professor Ma tin Snelgrove for his support and guidance during the course of my Ph.D. studies at Carleton University. I would also like to thank the members of my doctoral committee. Professcrs Miles Copeland. Tad Kwasniewski, Calvin Plett. and Garry Tarr for their advice.

It was a great pleast te to interact with members of my research group at Carleton University. Special thanks are due to Omid Shoaei, for sharing his knowledge on sigmadelta modulators through many technical discussions: Theo Varelas, for assisting me with chip layout and testing; Luc Lussier and Philip Lauzon for laying out test hoards: and Alaila Wirta, for helping me with administrative matters.

Most of all, I would like to thank my wife Jaleh for her love and companionship. my daughter Anahid for her cheerfulness which is a source of inspiration for me, and my mother for her endless support.

The financial and technical support of Nortel is gratefully acknowledged. I owe thanks to my past and present management at Nortel for instituting a research agreement for me. In particular, I am grateful to, Michael Krause. David Simmons, Kathy Howlett, Leonard McCready, Don Treen, David Agnew, David Lynch, and Jack Dyment. Thanks also to Robin Collins, microsurgeon at Nortel, for chip microsurgery and microphotographs. 


\section{Table of Contents}

\section{thstract}

Acknowledgments

Tahic of Contents

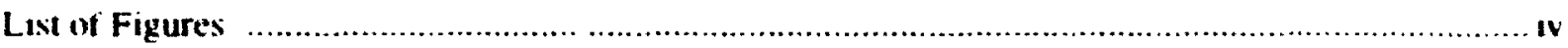

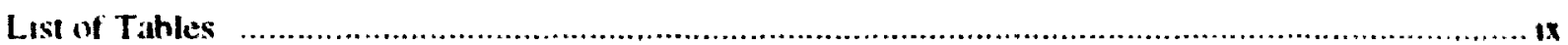

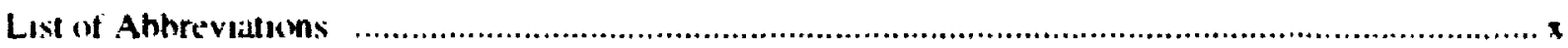

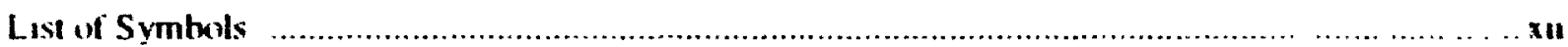

Introduction

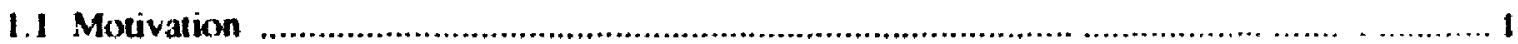

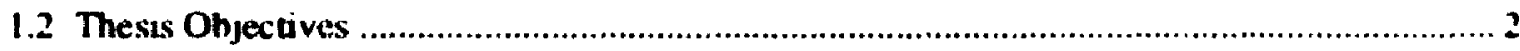

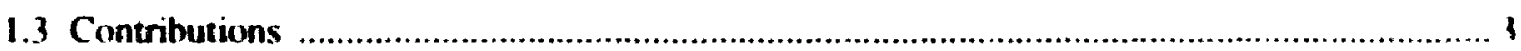

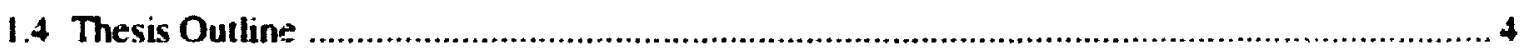

2 Impacts of CMOS Scaling on Performance of Mixed-Signal Circuits 6

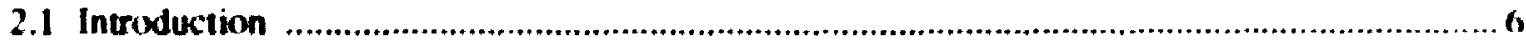

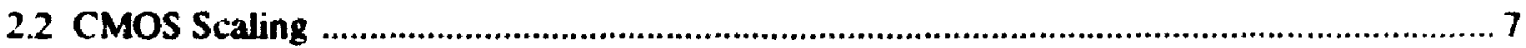

2.2.1 CMOS Scaling in the Next Decade ........................................................................

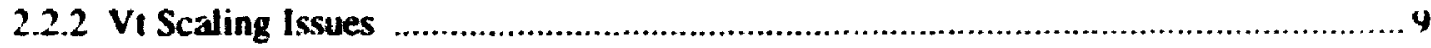

2.3 Low-Voltage Mixed-Signal Design .................................................................. 12

2.3.1 Limits on Digıtal CMOS Supply Voltage Scaling …............................................ 11

2 `.2 Limits on Analog CMOS Supply Voltage Scaling ............................................. If

2.3.3 Previous Work on Low-Voltage Digital Circuits ............................................... 14

2.3.4 Previous Work on Low-Voltage Analog Circuits ............................................ 20

2.3.4.1 Low-Voltage Active-RC Circuits ....................................................... 20

2.3.4.2 Low-Voltage Switched-Current Circuits .............................................. 21

2.3.4.3 Low-Voltage Switched-Capacitor Circuits ........................................... 22

2.3.5 Impact of Voltage Scaling on Power Dissipation of Mixed-Signal Circuits ........... 24

2.4 Oversampled Sigma-Delta Modulator Overview .............................................................. 28

2.4.1 Lowpass Sigma-Delta Modulacors ............................................................................ 30)

2.4.2 Bandpass Sigma-Delta Modulators ................................................................... 31

2.4.3 High-Speed SC Bandpass Sigma-Delo Modulator .......................................... 32 
29 Analug Circuil Design in a Standard Digital CMOS Process

2.5.1 SI Circuit Technique 35

2.5.2 High Performance Memory Cells 39

2.5.3 Linear SC Circuit Techniques in a Digital CMOS Process ...................................... 41

2.6 A Comparafive Siudy of SC and SI for Low-Voltage Applications ..................................... 46

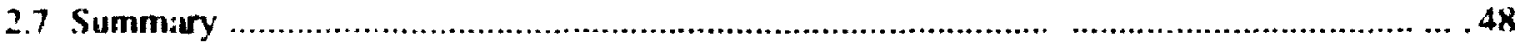

3 Low-Voltage SC Design with Low-Vt MOSFETs 50

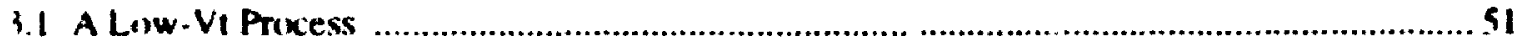

1.2 Curcuit Technique for Vi Reduction ....................................................................................4

3.1 Luw-Vi Transmission Gale Design for SC Circuits .............................................................57

3.4 Senes Transmission Gate and Composite Switch ................................................................. 63

3.4.1 Senes Transmission Gate Switch ..........................................................................65

3.4 .2 Composite Switch ...

1.5 A 2.25 V Second-Order Sigma-Delta Modulator ................................................................. 70

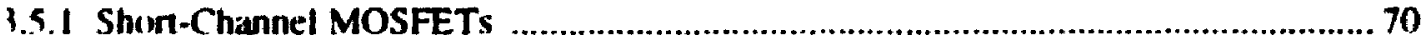

1.5.2 Shrtt-Channel MOSFETs Layout Issues ............................................................... 72

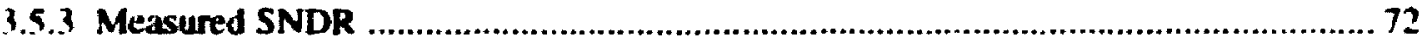

3.6 A I V First-Onder Sigma-Delta Modulator ............................................................................. 73

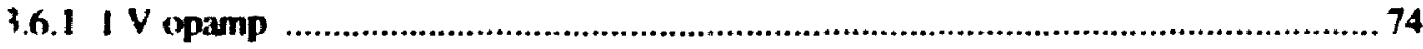

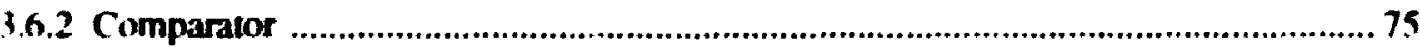

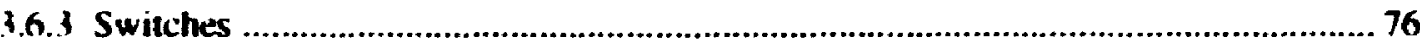

3.6.4 Measured Results for the 1 V Sigma-Delta Modulator ........................................... 77

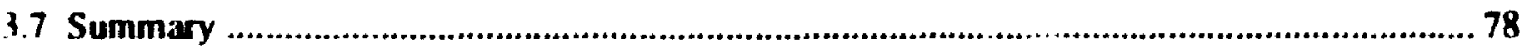

4 High-Speed SC Fourth-Order Bandpass Sigma-Delta Modulutor: 80

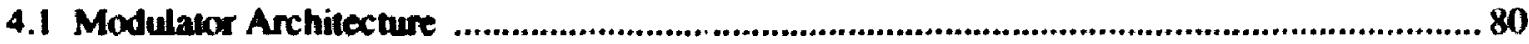

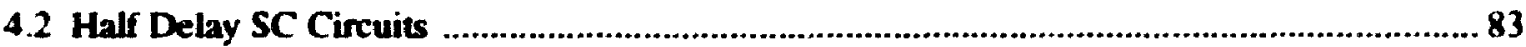

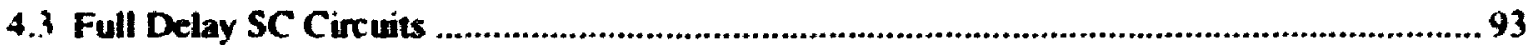

4.4 A SC Bandpass Sigma-Delea Modulator ................................................................. 101

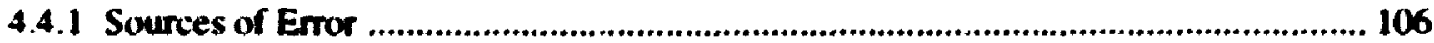

4.S A Double-Sampled SC Bandpass Sigma-Delta Modulator ................................................. 111

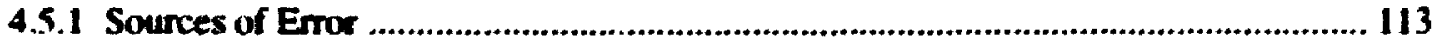

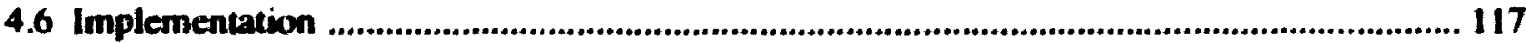

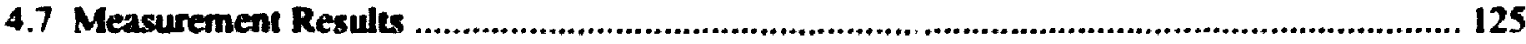


4.7.1 Fourth-Order Bandpass Sigma-Delta Modulator

4.7.2 Double-Sampled Fourth-Order SC Bandpacs Sigma-Delta Moululator

4.8 Summary

5 SC Bandpass Sigma-Delta Modulator Design In a Digital CMOS Process 1,30

S.1 MOS Capacitor 131

5.2 Distortion in SC Amplifier Caused by Non-linear Capaciturs 1 in

5.3 A SC Delay Circuit Using pMOSFET Capacitors 184

5.4 A Fourth-Order SC Bandpass Sigmi-Delta Modulahur Using pMOSFET Capaditon 141

5.4.1 Linear Charge Processor Viewpoint 145

5.5 Summary 146

6 Conclusions and Future Work

6.I Summary 147

6.2 Conclusions 144

6.3 Futire Work 149

Appendix A: MOSFET Equations

Appendix B: Natural MOSFET Characteristics

References 


\section{List of Figures}

Figure 2.1: A negative feedback $V_{1}$ adjust circuit .12

Figure 2.2: A basic CML inverter-buffer gate

Figure 2.3: A fully differenual CMOS Opamp

Fipure 2.4: Complementary transmission gate switch on-conductance for (a) $V_{D D}<2 V_{1}$. (b) $V_{D D}=2 V_{t}$. and (c) $V_{\mathrm{DO}}>2 \mathrm{~V}_{1}$. . .17

Figure 2.5: A simple current mirror cell .18

Figure 2.6: An active-RC integrator .21

Figure 2.7: A non-inverting SC integrator and its associated 2-phase non-overlapping clock .22

Figure 2.8: A switched-opamp SC integrator .24

Figure 2.9: CMOS invener .25

Figure 2.11: A block diagram of a is modulator .28

Figure 2.11: Equivalent linear system of Figure 2.10 .29

Figure 2.12: Pole/zero piacenseni of (a) a first-order lowpass $\Sigma \Delta$ and (b) a second-onder bandpass EA derived by $z^{-1} \rightarrow-z^{-2}$ transformation . .32

Figure 2.13: A weighted current mirror: . .36

Figure 2.14: A first generation memory cell .36

Figure 2.15: First generation SI integrator . .37

Figure 2.16: Second generation SI merr ory cell . .38

Figure 2.17: A second generation non-inverting lossless imegrator ........................................................38

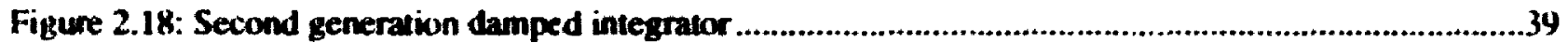

Figure 2.19: Regulated cascode memory cell.........................................................................................40

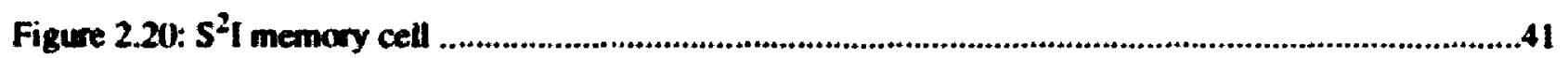

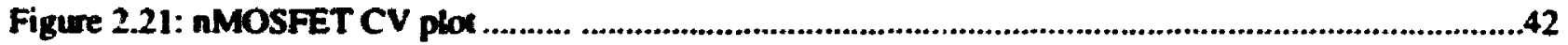

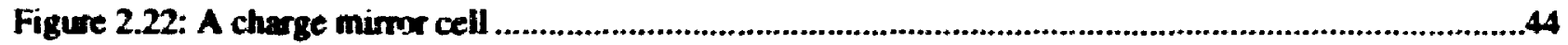

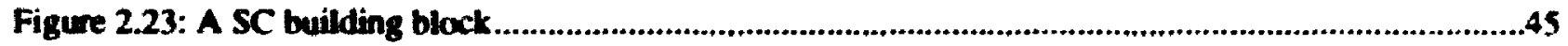

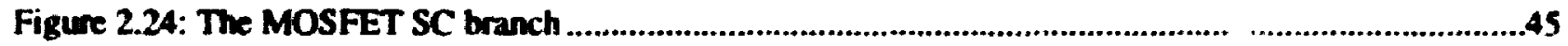

Figure 2.25: A SC circuit using the MOSFET SC branch....................................................................46

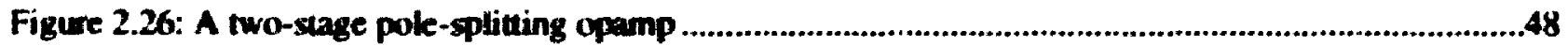

Figure 3.1: Gate-substrate woxfunction of nMOSFET and pMOSFET in an $n+/ p+$ dual poly gate CMOS

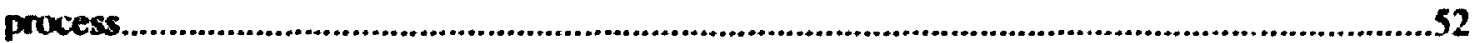

Figure 3.2: Measured $I_{D}(A)$ and $G_{m}(A V)$ versus $V_{G s}(V)$ for "naural" nMOSFET .............................53

Figure 3.3: Measured $\|_{D}(A)$ and $G_{m}(A V)$ versus $V_{G S}(V)$ for "natural" pMOSFET .............................53

Figure 3.4: Measured subthreshold characteristics of natural nMOSFET and pMOSFET ............................54 
Figure 3.5: Threshold voltage as a function of chaunel length.

Figure 3.6: Tumed-uff nMOSFET switch exhihiting (a) no punchihrught. (h) punchthrewgh due wo shan channel length. and (c) punihthrugh due to large drain wo surce puential ......................... \$n

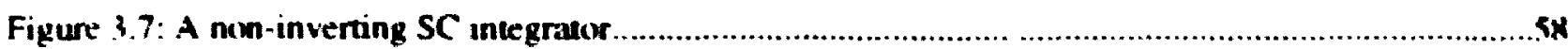

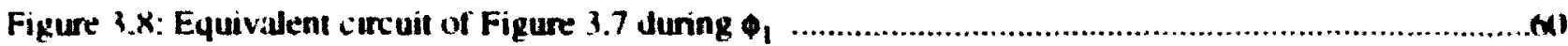

Figure 3.9: Equivalent circuit of Figure 3.7 during mon-owerlapping periud ..........................................2

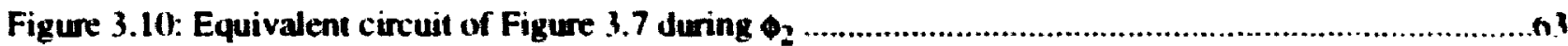

Figure 3.11: Measured ids $v s . v_{6}$ for an nMOSFET whth $v_{1}=1.30 \mathrm{mV}$ and $v_{s}=1$ ( solid line) and $v_{s}=11.2 v$ (doned line)

Figure 3.12: Tumed-off (a) nMOSFET and (b) pMOSFET switches ...................................................

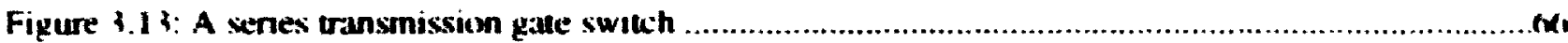

Figure 3.14: Leakage off-current through an nMOSFET switch (doned line) and a STG swilch (solid line) versus input voltage

Figure 3.15: On-ionductance of the nMOSFET (doned-line) and STG switch (solid-line) versus unpul signal

Figure 3.16: A composite switch .68

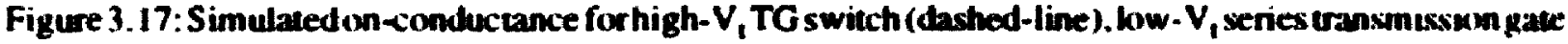
switch (doded-line). and the composite switch (solid-line) versus inpen signal

Figure 3.18: On-conductance for high-V, TG switch (dashed-line). kow-V, TG switch (dotted-line). and the composite switch (solid-line) versus inpu signal

Figure 3.19: Leakage current for high-V, TG switch (dashed-iine). low-V, TG switch (domed-line). and the composite switch (solid-line) versus input sigal

Figure 3.20: Drain current versus gate voltage for $\mathrm{a} 0.6 \mu \mathrm{m} n M O S F E T$ with $\left.v_{\mathrm{DS}}=1\right) .1 \mathrm{~V}$ (sulid line) and $v_{D S}=2 V$ (dashed line)

Figure 3.21: Dran off-current $\left(v_{G}=0\right)$ versus drain to suurce voltage for a $0.6 \mu \mathrm{mm} n M O S F E T$ with $v_{S B}=0$ (solid line) and $v_{S B}=0.5 \mathrm{~V}$ (dashed line) . .72

Figure 3.22: Measured IS modulator performance. . .73

Figure 3.23: A first-order SC $\Sigma \Delta$ modulator 74

Figure 3.24: A two-stage pole-splitting opamp .75

Figure 3.25: Schematic of the comparator. . .76

Figure 3.26: Simulated transmission gate switch resisance as a function of input signal .77

Figure 3.27: Simulated off-currem for the nMOSFET switch .74

Figure 3.28: Chip die microphotograph. .78

Figure 3.29: Measured spectrum (Vip $=420 \mathrm{mVpp}$ ) .79

Figure 4 1: A fourth-order double resonator bandpass is modulator $\$ 1$ 
Figure 4.2. Histograms of cutput levels for the first ( welid line) and the second (dashed line) resonator for

is -h dB tone input.....

Figure \& 3: Simulated output ypectrum of the founh-order handpass Is menlulator in Figure 4.1 for a sinusondial input. .82

Hapure 4 i kesmatox using delay cells .$\times 3$

Fyure \& 5 (a) A SC hall delay gain stape. (h) 2-phase non-overlapping clock, (c) input (dashed line) ind culpur (solid line) wavetorms .84

Figure 4.t: A ungle-ended equivalent circuil of Figure $4.5 \mathrm{~s}$ during (a) $\phi_{1}$ and (b) $\phi_{2}$ phases......................5

Fikure 4.7: Opamp in the closed-kon configuraton during

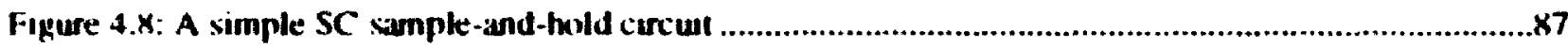

Fikure 4.4: Bottom-plate sampling technıque to elimınate signal dependent charge injextion......................88

Figure 4.10: Fully diflerential bottom-plate sampling to cancel fixed charge injection and clock texdutrough

4-1gure 4 11: Single-ended tequivalent circunts of Figure 4.5a during (a) sampling phase, and (b) holding phase .89

Figure 4.12: One capaciur sample-and-hold carcuit .92

Figure 4.1 3: A simple casciade SC delay circuit. .93

Figure 4.14: A SC delay gain stage .94

Figure 4.15: A single-ended equivalent circuit of Figure 4.14 during (a) $\phi_{1}$ and (b) phases .94

Figure 4.16: A double-sampked SC delay circuit. .97

Fipure 4.17: A sungle-tmiled cyuivalent circuit of Figure 4.16 during (a) $\phi_{1}$ and (b) $\phi_{2}$ phases .98

Figure 4. IX: Tworpath SC circuit and clock phases 100

Figure 4.19: Spectoum of the input signal (solid line) and the attenuated odd samples of the input signal (dotod line) 101

Figure 4.20: A SC resonator using two delay cells .102

Figure 4.21: A fourth-order SC bandpass sigma-delta modulator. 104

Figure 4.22: Worst case opamp loading for (a) bandpass $\Sigma \Delta$ of Figure 4.21 (b) bandpass $\Sigma \Delta$ modulator in [Long(x).3] 105

Figure 4.23: Output spectrum from Eldo simulation 105

Figure 4.24: Output spectrum of the fourth-order bandpass $\Sigma \Delta$ modulator with an opamp gain of $40 \mathrm{~dB}$ and cpamp inpus capacitance of $\mathrm{C}_{\mathrm{m}}=\mathbf{2 C _ { u }}$

Figure 4.25: SNR kss versus OSR / HR, from analytical result (solid line) and from simulation results (o pounts).

Fipure 4.26: A duhle-sampled SC resunator

Figure 4.2X: Output spectrum from Eldo simulation. 
Figure 4.27: A double-sampled SC lourth-order hindpass sigmi-delta moxdulilir

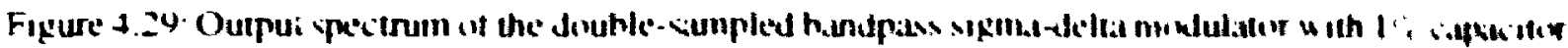

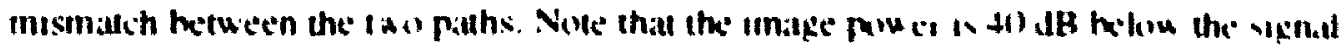
prwatr.

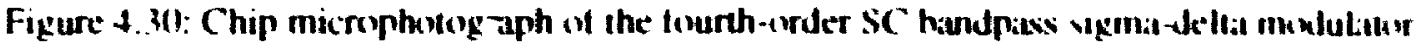

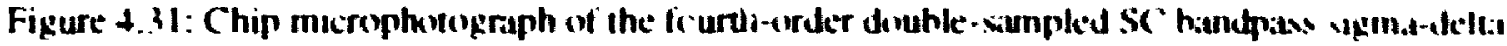
modulator.

Figure 4.32: Transmission galte switch on-resistance vs. stgnid level

Figure 4. 33: Fully differentad caside opamp

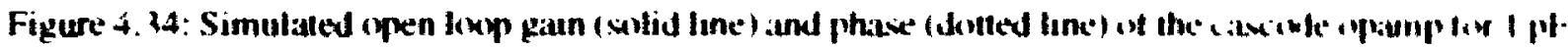
low capaciance

Figure 4.35: Test suncture for settling Ime sumulition

Figure 4.36: Worst cisse settling unie simulaum in Elute.

Figure 4.37: Fully differential comparatur

Figure 4.38: Measured output speconm of the rourth-order handpass sigma-delta moxulutu for an inpul signal of 10 dB helow full scale.

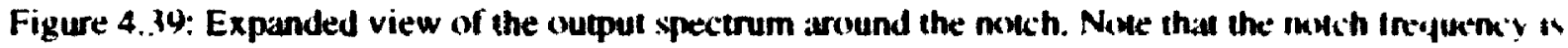
shifted by about $5(x) \mathrm{kHz}$ w the left (kwer frequency)

Figure 4.40: Measured ouput spectrum of the double-sampled forth-1rder SC hamplass stgina-delta inodulator for an input signal of $10 \mathrm{~dB}$ helow fis! scake. Nove that mage signal is itwiut 39 aB below the fundamental signal.

Figure 4.41: Expanded view of the output spactum around the axtch. Note that the nhch irequitic is shifted by ahout $1 \mathrm{MHz}$.

Figure 5. 1: Structure of (a) a MOS capacitor and (b) an nMOSFET transistur

Figure 5.2: Analytical C-V characteristics of an nMOSFET transistor.

Figure 5.3: Measured (solid line) and simulated (dashed line) C-V plox of in nMOSFET

Figure 5.4: Measured (solid line) and simulated (dashed line) C-V plox of a pMOSFET

Figure 5.5: Simulated C-V characteristics of a hw-VI nawral nMOSFET (dashed lune ) and a thresh ild adjusted nMOSFET (solid line) ................................................................ I if,

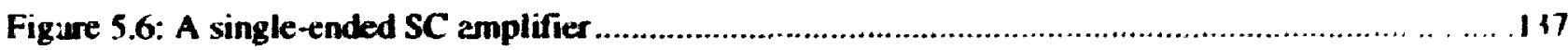

Figure 5.7: SC delay cell using pMOSFET capacıurs....................................................... I4)

Figure 5.8: Output spectrum of the fully differentual SC gan stage. Figure 5.7. during $*, \ldots \ldots \ldots \ldots \ldots \ldots .14$

Figure 5.9: Output spectrum of the fully differential SC unity gan stage. Figure 5.7. Junng $\oplus_{1} \ldots \ldots \ldots \ldots 142$

Figure 5.10: A fourth-order bandpass sıgma-delta modulator usung pMOSFET capacitors .............. 14 \$

Figure 5.11: Output spectrum of the fourth-order SC bandpass sigma-delta modulalle of Figure 5.10 
for an input signal of $6 \mathrm{~dB}$ below full scale 144

Figure 5.12: A fully differential cascode opamp 144

Figure B.1: Measured $I_{D}(A)$ and $G_{m}(A V)$ versus $V_{G S}(V)$ for "natural" $n M O S F E T$. 154

Figure B.2: Measured $I_{D}(A) l$ and $G_{m}(A / V)$ versus $V_{G S}(V)$ for "natural" pMOSFET. .154

Figure B.3: Measured nMOSFET $I_{D S}-V_{D S}$ characteristics. $I_{D S}-\left(V_{G S}-V_{v}\right)^{1.27}$ 155

Figure B.4: Measured pMOSFET $I_{D S}-V_{D S}$ characteristics. $I_{D S}-\left(V_{G S}-V_{\nu}\right)^{1.32}$ 155 


\section{List of Tables}

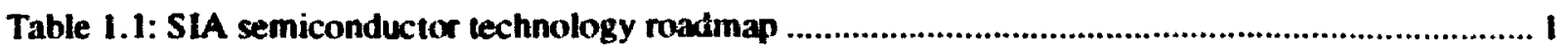

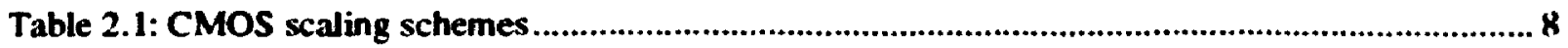

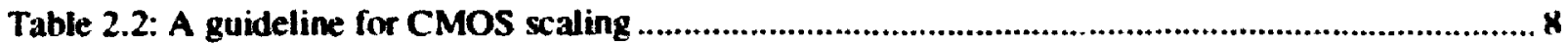

Table 3.1: On-resistance of switches with $W=10 \mu \mathrm{mn}$............................................................................ 71

Table 3.2: Dimension of MOSFETs used in the opamp of Figure 3.24 ............................................ 74

Table 3.3: Simulated opamp performance for a I pF load capacitance................................................ 75

Table 3.4: Transistor sizes for the comparator .....................................................................................

Table 4.1: Some MOSFET parameters in HCMOS5a process...................................................... $11 \mathrm{~K}$

Table 4.2: Target specification for the bandpass $\Sigma \Delta$ modulator .................................................... I19

Table 4.3: Transistors sizes used in the opamp .......................................................................121

Table 4.4: Transistor sizes used in the CMFB circuit ......................................................................... 121

Table 4.5: Simulated characteristics of opamp for I pF load capacitance ......................................... 124

Table 4.6: Comparator transistor sizes ................................................................................................ 125

Table 5.1: nMOSFET capacitance voltage coefficients ................................................................... 135

Table 5.2: pMOSFET capacitance voltage coefficients .................................................................. 135

Table 5.3: Simulated characteristics of opamp for 1pF load capacitance ........................................... 145 


\section{List of Abbreviations}

AVD

ASIC

BiCMOS

CE

CML

CMOS

CV

DC

DR

DRAM

FE

$H D_{k}$

IF

LDI

LDD

LT

MOSFET

NTF

nMOSFET

opamp

OSR

OTA
Analog-to-Digi. l

Application Specific Integrated Circuit

Bipolar and Complementary Metal Oxide Semiconductor

Constant Electric Field

Current Mode Logic

Complementary Metal Oxide Semiconductor

Constant Voltage

Direct Current

Dynamic Range

Dynamic Random Access Memory

Forward Euler

$k^{\text {th }}$ harmonic distortion

Intermediate Frequency

Lossless Discrete Integrator

Lightly Doped Drain

Low Temperature

Metal Oxide Semiconductor Field Effect Transistor

Noise Transfer Function

n-channel Metal Oxide Semiconductor Field Effect Transistor

operational amplifier

Oversampling Ratio

Operational Transconductance Amplifier 


$\begin{array}{ll}\text { pMOSFET } & \text { p-channel Metal Oxide Semiconductor Field Effect Transistor } \\ \text { QCV } & \text { Quasi-Constant Voltage } \\ \text { SC } & \text { Switched-Capacitor } \\ \text { SCE } & \text { Short-Channel Effect } \\ \text { SI } & \text { Switched-Current } \\ \text { SNR } & \text { Signal to Noise Ratio } \\ \text { SNDR } & \text { Signal to Noise+Distortion Ratio } \\ \text { STF } & \text { Signal Transfer Function } \\ \text { STG } & \text { Series Transmission Gate } \\ \text { TG } & \text { Transmission Gate } \\ \text { TTL } & \text { Transistor-Transistor Logic } \\ \text { UGBW } & \text { Unity-gain Bandwidth } \\ \text { VLSI } & \text { Very Large Scale Integration } \\ \text { IA } & \text { Sigma-Delta }\end{array}$




\section{List of Symbols}

\begin{tabular}{ll} 
II & Absolute value \\
A & opamp DC gain \\
$C_{g b}$ & Gate-bulk capacitance of MOSFET \\
$C_{g s}$ & Gate-source capacitance of MOSFET \\
$C_{o x}$ & Specific gate capacitance of MOSFET \\
$E_{c}$ & Conduction band edge \\
$E_{g}$ & Silicon bandgap energy \\
$E_{v}$ & Valence band edge \\
$e$ & Quantization noise \\
$f$ & Frequency in Hz \\
$f_{0}$ & Center frequency \\
$f_{s}$ & Sampling frequency \\
$g_{d s}$ & Drain-source conductance \\
$g_{m}$ & MOSFET transconductance \\
$H(s)$ & $s$-domain transfer function \\
$H(z)$ & $z$-domain transfer function \\
$I_{d s}$ & Drain current \\
$i_{i}$ & Input current \\
$i_{o}$ & Output current \\
$i_{o f f}$ & MOSFET subthreshold off-current \\
$\boldsymbol{k}$ & CMOS technology scaling factor \\
$\boldsymbol{H}$ & Boltzman's constant \\
\hline $\boldsymbol{H}$ &
\end{tabular}


$L \quad$ MOSFET channel length

$m(\omega) \quad$ Magnitude error frequency response

$N_{A} \quad$ Acceptor impurity density

$n$

Subthreshold slope factor

$n_{0} \quad$ Quantization noise in signal band

$\hat{Q} \quad$ Fixed charge density

$R_{\text {on }} \quad$ MOSFET drain-source on-resistance

$r_{j}$

MOSFET junction depth

$S \quad$ Subthreshold swing factor

$\left.S\right|_{y} ^{x} \quad$ Sensitivity of $x$ to variation in $y$

$T \quad$ Clock period

$T \quad$ Temperature

$t_{D} \quad$ Delay time

$t_{\text {ox }} \quad$ Gate oxide thickness

$V_{B E} \quad$ Base-Emitter voltage

$V_{D D} \quad$ Power supply voltage

$V_{F B} \quad$ Flat-band voltage

$V_{i c m} \quad$ Input common-mode voltage

$V_{\text {ocm }} \quad$ Output common-mode voltage

$V_{\text {on }}$ MOSFET gate over-drive voltage (on-voltage)

$v_{t} \quad$ MOSFET threshold voltage

$V_{i n} \quad$ nMOSFET threshold voltage

$V_{t p} \quad$ PMOSFET threshold voltage

$\bar{v}_{n} \quad$ RMS noise voltage 


\begin{tabular}{|c|c|}
\hline$v_{D}$ & Drain voltage \\
\hline$v_{\imath d}$ & Differential input voltage \\
\hline$v_{\text {in }}$ & Negative input voltage \\
\hline$v_{i p}$ & Positive input voltage \\
\hline$v_{\text {od }}$ & Differential output voltage \\
\hline$v_{\text {on }}$ & Negative output voltage \\
\hline$v_{o p}$ & Positive output voltage \\
\hline$v_{s}$ & Source voltage \\
\hline$w$ & MOSFET channel width \\
\hline$z^{-1}$ & Sampled-data representation of delay $\left(z^{-1}=e^{-j \omega T}\right)$ \\
\hline$\alpha$ & Velocity saturation index \\
\hline$\alpha_{1}$ & First-order voltage coefficient of capacitance \\
\hline$\alpha_{2}$ & Second-order voltage coefficient of capacitance \\
\hline $\boldsymbol{\beta}$ & MOSFET transconductance parameter $\left(\beta=\mu C_{o x} W / 2 L\right)$ \\
\hline $\boldsymbol{\beta}$ & Feedback factor \\
\hline$\varepsilon_{o x}$ & Oxide dielectric constant \\
\hline$\varepsilon_{s i}$ & Silicon dielectric constant \\
\hline$\phi_{B}$ & Bulk potential \\
\hline$\phi_{F}$ & Fermi potential \\
\hline$\phi_{m s}$ & Metal-silicon work function difference \\
\hline$\phi_{n}$ & Clock phase $n$ \\
\hline$\phi$, & Thermal voltage \\
\hline$\gamma$ & Body effect coefficient \\
\hline $\boldsymbol{\mu}$ & Mobility \\
\hline
\end{tabular}


$\mu_{n} \quad$ Electron mobility

$\mu_{p} \quad$ Hole mobility

$\theta(\omega)$ Phase error frequency response

$\omega_{u} \quad$ Unity-gain bandwidth

$\omega_{0} \quad$ Cut-off frequency $(-3 d B)$ 


\section{Chapter 1}

\section{Introduction}

\section{I Motivation}

Higher speed and higher density are the main benefits of CMOS VLSI technology and are achieved by shrinking the feature size of the MOSFET devices. In the past, Constant Voltage (CV) scaling has been used for CMOS (down to $0.5 \mu \mathrm{m}$ ) to achieve higher performance and to maintain supply voltage compatibility. However, as MOSFET miniaturization reaches to deep submicron sizes ( $0.5 \mu \mathrm{m}$ and below), supply voltage xaling based on Quasi-Constant Voltage (QCV) scaling must be adopted to assure reliability [Kakumu90]. Hot carrier effects and gate oxide breakdown are the two important reliability factors determining how high power supply voltage can be. The Semiconductor Industry Association (SIA) roadmap (Table 1.1) predicts a supply voltage of $0.9 \mathrm{~V}$ for semiconductors by the year 2010 [SIA94].

\begin{tabular}{|c|c|c|c|c|c|c|}
\hline Year & 1995 & 1998 & 2001 & 2004 & 2007 & 2010 \\
\hline \hline Feature size $(\mu \mathrm{m})$ & 0.35 & 0.25 & 0.18 & 0.13 & 0.1 & 0.07 \\
\hline Power supply voltage (V) & 3.3 & 2.5 & 1.8 & 1.5 & 1.2 & 0.9 \\
\hline
\end{tabular}

Table 1.1: SIA semiconductor technology roadmap

The move toward lower supply voltages is also fueled by low-power battery powered portable devices [Thomas93]. An ideal power supply for a battery powered system is a single-cell $(1.2 \mathrm{~V}-0.9 \mathrm{~V})$ off-the-shelf battery. 
Integrated circuits are moving increasingly into the mixed-signal world where more parts of a system are implemented on a single chip. Performance and density of digital circuits are expected to follow the famous Moore's law-achieving a $2 \mathrm{X}$ increase in circuit density and a slower rate of (1.4-1.2)X increase in circuit speed every three years. Energy per operation in digital circuits $\left(E-\mathrm{Cl}^{2}\right)$ is also reduced due to supply voltage sialing in deep submicron CMOS. However, lowering the supply voltag firectly reduces the signal swing in analog circuits, which in turn makes the design of wide dynamic range mixedsignal circuits a challenge. This raises the following question:

- Will mixed-signal supply voltage follow the digital supply vollage down to I V?

In deep submicron CMOS $(L<0.5 \mu \mathrm{m})$, the charge carriers" velocity will be saturated at the pinch-off point in the channel and as a result the classical yeadratic saturation current becomes $I_{d s a t}-\left(v_{i j S}-V_{1}\right)^{\alpha}$, where $\alpha=1.3$ to 1.4 instead of 2 [HuY4|. Therefore. $1.4 X$ circuit speed improvement every generation will slow down to about $1.2 X$. leading to another question:

- What are the circuit techniques to maintain a high dynamic range at high speed?

Analog circuits are usually implemented using Switched-Capacitor (SC) techniques because of their high circuit accuracy. Traditionally. SC circuits are implemented in analog CMOS processes where linear double poly capacitors are available. However, the driving forces behind the CMOS technologies are DRAMs and microprocessors which do not require a double-poly process. The next question is:

- Can linear SC circuits be implemented in a standard digital CMOS process?

These questions form the core of the research conducted in this thesis.

\subsection{Thesis Objectives}

This thesis is concerned with the three key issues mentioned above, namely: low voltage. high speed, and the implementation of linear SC circuits in a digital CMOS process. Through analysis and some preliminary experimental circuits it will be shown that SC: mixed-signal circuits will scale down to $1 \mathrm{~V}$.

Low voltage: To demonstrate the feasibility of $1 \mathrm{~V}$ mixed-signal circuits. Jow-threshold 
MOSFET transistors are required. A $1 \mathrm{~V}$ CMOS process technology is developed as a subset of a $11.5 \mu \mathrm{m} \mathrm{n} / \mathrm{p}^{+}$dual poly gate CMOS process. In this process, natural threshold voltage MOSFETs are optimized to have a $V$, of about $200 \mathrm{mV}$. A first-order voice-band $\mathrm{SC} \Sigma \Delta$ modulator is implemented in this process and operates at $1 \mathrm{~V}$ power supply.

High speed: Low-voltage operation is known to compromise speed unless the technology thrink is in proper relation to the supply voltage. Two new high-speed bandpass $\Sigma \Delta$ modulators are implemented in a $3 \mathrm{~V} 0.5 \mathrm{\mu m}$ CMOS process to verify their performance and functionality.

Linear SC design in a digital CMOS process: MOSFET capacitors biased in strong inversion or accumulation regimes can be used to replace linear capacitors. A telescopic opamp with different input and output common mode voltages is shown to be suitable for this purpose. Also described is the design of a fourth-order SC bandpass $\Sigma \Delta$ modulator using pMOSFET transistors, biased in strong inversion, as linear capacitors.

The benchmark circuits are chosen to be $\Sigma \Delta$ modulators for the following two reasons. First, a $\Sigma \Delta$ modulator is a typical mixed-signal circuit with enough complexity to test the technology and to demonstrate the achievable performances. It contains important analog cells such as opamps. comparators and switches as well as some digital gates including inverters. NORs, and a D-type fip-flop. Second, $\boldsymbol{\Sigma} \Delta$ modulators are widely used as precision Analog-to-Digital (A/D) converters and are becoming popular in A/D conversion of narrow-band signals at Intermediate Frequencies (IF) in radio receiver circuitry.

\subsection{Contributions}

Contributions of this work include:

- Proposed and verified suitability of low- $V$, natural MOSFETs for $1 \mathrm{~V}$ mixed analog/ digital applications.

- Compared Switched Capacitor (SC) and Switched Current (SI) analog techniques for low-voltage applications.

- Analyzed low-voltage SC circuits using low-, MOSFETs. 
- Proposed low-leakage series transmission gate and composite switches.

- Demonstrated a technique for exploiting the short-channel effects to obtain louvoltage switches.

- Implemented a high-speed architecture for bandpass $\Sigma \Delta$ modulator.

- Developid a novel double-sampled bandpass $\mathbf{\Sigma} \Delta$ modulator.

- Designed a fourth-order SC bandpass $\Sigma \Delta$ modulator in a standard digital ( MOS process.

\subsection{Thesis Outline}

Chapter I provides an introduction.

Chapter 2 starts with an examination of CMOS scaling in deep submicron geometries which require supply voltage sualing down to $1 \mathrm{~V}$. Theoretical limits on low-voltage digital and analog CMOS due to circuit topology are discussed and some recent work in this area is reviewed. Oversampling and noise-shaping are then brietly overviewed and a simple mapping of lowpass $\Sigma \Delta$ to bandpass $\Sigma \Delta$ [Jantzi] is described. High-speed SC bandpass $\Sigma \Delta$ modulator techniques are also presented. Next. the Switched-Current (SI) analog circuit technique [Hughes89] is introduced and methods of implementing SC circuits in a digital CMOS process are discussed. Finally. a comparative study of SC and SI for low-voltage applications is presented. It is concluded that the SC technique is preferred in low-voltage circuits from a dynamic range point of view.

In Chapter 3, low-voltage SC circuit design using low-V, MOSFETs is considered. First. two methods of achieving low- $V$, MOSFETs in current CMOS prociesses aje proposed. The impact of subthreshold leakage current on the accuracy of the SC circuit is then analyzed. Methods of reducing the subthreshold leakage through analog switches are discussed next and two new switch topologies (series transmission gate and composite switch) addressing this problem are presented. Finally, two experimental circuits, a $2.25 \mathrm{~V} \Sigma \Delta$ modulator using short-channel MOSFETs and a 1 V $\Sigma \Delta$ modulator using low$V$, natural MOSFET are discussed.

Chapter 4 begins by examining motivations for a high-speed bandpass $\Sigma \Delta$ modulator. A 
Chapter I- Introduction

5

high-speed fourth-order SC bandpass $\Sigma \Delta$ modulator is discussed, followed by presentation of a double-sampled SC bandpass IS modulator. Fully differential circuit implementation of both modulators in a $3 \mathrm{~V} 0.5 \mu \mathrm{m}$ analog CMOS process are described and measured results are presented.

Chapter 5 describes a SC circuit technique employing MOSFET capacitors biased in strong inversion as linear capacitors. Distortion caused by capacitor non-linearity in a SC amplifier is analyzed. Then, the design of a fourth-order bandpass $\Sigma \Delta$ modulator using a pMOSFET transistor as a linear capacitor is described.

Chapter 6 draws the thesis conclusions and proposes future work.

Appendices which provide some additional information on MOSFET equations used in this thesis and measured characteristics of natural MOSFETs are also included. 


\section{Chapter 2}

\section{Impacts of CMOS Scaling on Performance of Mixed-Signal Circuit.;}

\subsection{Introduction}

The sealing of CMOS technology in the deep submicron regime is expected to continue providing faster and denser devices. In section 2.2. CMOS scaling is reviewed along with the fact that, for device reliability, the supply voltage is being scaled down in deep submicron technologies. The power supply voltage of $0.1 \mu \mathrm{m}$ CMOS technology is expected to be about I V [Tavari95]. Along with the power supply. the threshold voltage of MOSFETs must be scaleu 'own to achieve high-speed circuits. Limits on supply voltage scaling for mixed-signal circuits are considered in section 2.3; some current research in the area of low-voltage digital and analog circuits is also presented. The impact of reducing the supply voltage on power dissipation of digital and analog circuits is

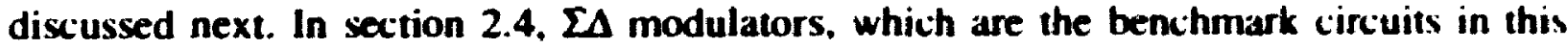
thesis, are introduced and high-speed bandpass SC $\Sigma \Delta$ modulators repuned in the literature are discussed. Most advanced CMOS processes are developed to suppon memory and digital circuits. Thus. mis.ed-signal circuits that are realizable in a standard digital CMOS process are desired. Analog SI and SC circuit techniques that allow mixedsignal circuits to be implemented in a digital CMOS process are discussed in section 2.5 . Finally, a comparative study of SC and SI sampled-dat? analog circuit techniques is presented in section 2.6. It will be seen that SC has advantages over SI for low-voltage design. 


\subsection{CMOS Scaling}

The scaling of CMOS technology is achieved by reducing the dimensions of MOSFET transistors by a factor of $k(k>1)$. The orig.nal Constant Electric-field (CE) scaling law proposed by Dennard [Dennard74] involved supply voltage scaling as well as scaling all the device dimensions to preserve the same electric-field in the scaled-down device. A constant electric-field in the substrate is obtained by increasing device-well doping concentrations in the smaller devices. The CE scaling scheme improves the device density by $k^{2}$, reduces the gate delay by $k$, and lowers power dissipation by $k^{2}$ thus maintaining a constant power density.

Drawbacks of CE scaling are the departure from standard power supply voltage, problems with MOSFET threshold voltage scaling, and degradation in analog signal swing. Therefore, in the past (down to $0.5 \mu \mathrm{m}$ technology) a Constant Voltage (CV) scaling rule has been used for CMOS to maintain a supply voltage of $5 \mathrm{~V}$ and to achieve higher circuit performance; gate delay is reduced by $k^{2}$ in a CV scaling. However, in CV scaling the higher electric field causes a reliability hazard due to hot carriers and gate oxide breakdown. A higher electric field in deep submicron devices also brings about carrier velocity saturation, which causes speed improvement to become linearly proportional to $k$ (similar to CE scaling). Another drawback of $\mathrm{CV}$ scaling is an increase in power dissipation density which is proportional to $k^{3}$.

A compromise between CV and CE scaling schemes is a general scaling theory called Quasi-Constant Voltage (QCV) scaling. In a QCV scaling, power supply voltage is reduced at a slower rate of approximately $\sqrt{k}$. In Table 2.1, various scaling schemes and their effects on MOSFET device characteristics are summarized.

Studies of CE, CV, and QCV scaling for mixed-signal circuits in high-micton [Wong83] and submicron [Sano88] CMOS technologies have shown that QCV scaling is ciose to an optimum scaling scheme.

\subsubsection{CMOS Scaling in the Next Decade}

Recently, Davari published a guideline for CMOS scaling in the next decade for digital circuits [Davari95]. A summary of this guideline is produced in Table 2.2. Two different 


\begin{tabular}{|c|c|c|c|}
\hline Parameters & CE & CV & QCV \\
\hline Lateral dimensions (L) & $1 / k$ & $1 / k$ & $1 / k$ \\
\hline Gate oxide thickness $\left(t_{0 x}\right)$ & $1 / k$ & $1 / k$ & $1 / \sqrt{k}$ \\
\hline Doping concentration & $k$ & $a^{2}$ & $k^{1.5}$ \\
\hline Supply voltage (V) & $1 / k$ & 1 & $1 /(\sqrt{k})$ \\
\hline Electric field & 1 & $k$ & 1 \\
\hline Current (I) & $1 / k$ & $k$ & $1 / k$ \\
\hline Area (A) & $1 / k^{2}$ & $1 / k^{2}$ & $1 / k^{2}$ \\
\hline Capacitance $\left(C=A \varepsilon / t_{o x}\right)$ & $1 / k$ & $1 / k$ & $1 / k^{1.3}$ \\
\hline Gate delay (VCI) & $1 / k$ & $1 / k^{2}$ & $1 / k$ \\
\hline Power dissipation (VI) & $1 / k^{2}$ & $k$ & $1 / k^{1.3}$ \\
\hline Power density (VI/A) & 1 & $t^{3}$ & $\sqrt{k}$ \\
\hline Energy Jissipation $\left(\mathrm{CV}^{2}\right)$ & $1 / k^{3}$ & $1 / k$ & $k^{2 x}$ \\
\hline
\end{tabular}

Table 2.1: CMOS scaling schemes

power supply voltages are recommended for each generation of deep submicron technology: one for high-perfornance (HP) circuits and another one for low-power (LP) circuits.

\begin{tabular}{|l|l|l|l|l|}
\hline \multicolumn{1}{|c|}{ Year } & \multicolumn{1}{|c|}{1995} & \multicolumn{1}{|c|}{1998} & \multicolumn{1}{|c|}{2001} & \multicolumn{1}{c|}{2004} \\
\hline \hline Supply voltage $(H P / L P) \mathrm{V}$ & 3.312 .5 & $2.5 / 1.5$ & $1.5 / 1.0$ & 1.211 .0 \\
\hline Channel length $(\mu \mathrm{m})$ & 0.35 & 0.25 & 0.18 & 0.13 \\
\hline Oxide thickness $(\mathrm{nm})$ & 9 & 6 & 3.5 & 2.5 \\
\hline
\end{tabular}

Table 2.2: A guideline for CMOS scaling

This guideline is in agreement with the SIA technology roadmap for semiconductors presented in Chapter 1. By the year 2004, the power supply voltage is predicted to be scaled down to $1.2 \mathrm{~V}$ for high-performance circuits and $1 \mathrm{~V}$ for low power applications. Supply voltage scaling in CMOS, undertaken to maintain reliability, causes severe speed performance degradation unless the MOSFET threshold voltage $\left(V_{t}\right)$ is scaled down. 
Circuit speed suffers because it is proportional to transconductance $\left(g_{m}\right)$ and for a fully turned- on MOSFET (i.e., $v_{G S}=V_{D D}$ ) the transconductance is $g_{m}-V_{D D}-V_{t}$.

\subsection{2 $V_{t}$ Scaling Issues}

A MOSFET transistor biased below threshold voltage $\left(v_{G S} \leq V_{t}\right)$ operates in the weak inversion mode where drain current is exponentially dependent on the value of $v_{G S}-V_{t}$ (Appendix A). The subthreshold off-current (for $v_{G S}=0$ ) is given by the following simplified equation:

$$
i_{\text {off }}=I_{D 0} \frac{W}{L} 10^{-V_{1} / S} \text {, }
$$

where $S$ is the subthreshold swing and is given by

$$
S=n \frac{k T}{q} \ln (10)
$$

and typically $n \approx 1.4$.

Non-Scalability of Subthreshold Swing

As can be seen from (2-2), subthreshold swing does not scale with technology scaling. Therefore, the main limitation of $V_{l}$ scaling is due to the non-scalability of $S$.

At room temperature, $S$ has a typical value of $80 \mathrm{mV} /$ decade for bulk CMOS, and increases to about $100 \mathrm{mV} /$ decade at $85^{\circ} \mathrm{C}$. Reducing the threshold voltage of MOSFETs by $100 \mathrm{mV}$ increases the subthreshold off-current by an order of magnitude. Higher offcurrent increases the standby power dissipation in digital circuits and limits the accuracy of the analog SC circuits.

Digital circuits: In digital CMOS circuits the total power consumption expression is

$$
P=p_{0 \rightarrow 1} f C_{L} v_{D D}^{2}+i_{o f f} V_{D D}+i_{S C} V_{D D},
$$

where $p_{0 \rightarrow 1}$ is the activity factor, $f$ is the clock frequency, $C_{L}$ is the load capacitance, $V_{D D}$ is the supply voltage, and $i_{s c}$ is the short circuit current during each input transition when both nMOSFET and pMOSFET are temporarily on.

Lowering the $V_{t}$ increases the off-current and the second term in the above equation will 
rise. However, low- $V$, MOSFETs allow operation at a lower $V_{D D}$ without sacrificing speed performance. A reduced $V_{D D}$ decreases the dynamic power consumption, the first term in (2-3).

Several studies [Liu93] [Burr91] have shown that a significant power saving is achieved by operating at very low supply voltages (as low as $200 \mathrm{mV}$ ) using low- $V$, MOSFETs. while preserving circuit speed.

Analog circuits: Analog switches in SC and SI circuits must exhibit a low on-resistance when closed and very low leakage current when opened. At a low supply voltage. low- $V$, MOSFET are suitable as switches due to their low on-resistance. However. low- $V$, switches are leaky and limit the precision of the analog circuits. In Chapter 3, the impact of leakage through analog switches is analyzed and some solutions are proposed.

A sharper subthreshold slope (i.e., a lower value of $S$ ) is obtained by reducing $\boldsymbol{n}$. or $\boldsymbol{T}$. or both in (2-2). The lowest possible value for $n$ is achieved in a CMOS on Silicon-onInsulator (SOI) process where $n=1$. The corresponding value of $S$ is $57.5 \mathrm{mV} /$ deciade at room temperature. Low-Temperature (LT) CMOS is another method of scaling the subthreshold swing. Operation at liquid nitrogen temperature $(77 \mathrm{~K})$ reduces $S$ by a factor of four to about $14.5 \mathrm{mV} /$ decade.

Both SOI and LT-CMOS furnish a better $v_{t}$ scaling and are promising technologies for very low-voltage deep sub-0.1 $\mu \mathrm{m}$ CMOS processes.

\section{Threshold Voltage Fluctuation}

Another limitation of $V_{t}$ scaling is based on the fundamental variability of the threshold voltage due to process variations. Fluctuation in $V_{t}$ is mainly due to random dopant and channel length variations. For a normally distributed threshold voltage, the standard deviation due to random dopant concentration variation is

$$
\delta V_{t}=\frac{q}{2 C_{O x}} \sqrt{\frac{\pi N_{A}}{2}}(W L)^{-3 / 8}\left(X_{D}\right)^{1 / 4}
$$

where $N_{A}$ is the doping density, $L$ and $W$ are the channel length and width of the transistor, and $X_{D}$ is the depletion width [Keyes75]. 
Recent studies based on measurements [Mizuno93] and simulations [Nishinohra92] [Wong93] show that $V$, variation due to dopant fuctuation is proportional to $1 / \sqrt{L}$, in agreement with (2-4), and increases as channel length is reduced. In [Nishinohra92], 2-D and 3-D simulations indicate that $\delta\left(\Delta V_{p}\right)$ is expected to increase from $10 \mathrm{mV}$, for $1 \mu \mathrm{m}$ technology, to about $30 \mathrm{mV}$ for a $0.1 \mu \mathrm{m}$ technology.

Temperature variation also changes the $V$, by approximately $1.25 \mathrm{mV} /{ }^{\circ} \mathrm{C}$ [Chen90]. For an industrial temperature range of $-50^{\circ} \mathrm{C}$ to $85^{\circ} \mathrm{C}$, the total threshold shift tue to temperature alone is about $170 \mathrm{mV}$.

In low- $V$, processes, threshold voltage changes due to process variations and temperature have a big impact on speed and standby power dissipation. For instance speed performance variation (based on quadratic equation formulae given in Appendix $A$ ) due to a $\pm 100 \mathrm{mV}$ change in $V_{t}$ is about $\pm 2.5 \%$ in a $5 \mathrm{~V}$ process with $V_{t}=0.8 \mathrm{~V}$, and rises to $\pm 12 \%$ in a $1 \mathrm{~V}$ process with $V_{t}=0.2 \mathrm{~V}$. Assuming $i_{D S}=1 \mu \mathrm{A}$ at $v_{G S}=V_{t}$ and $S=100 \mathrm{mV} /$ decade, the highest off-current in the above example would be $100 \mathrm{fA}$ for the $5 \mathrm{~V}$ high- $V$, and $10 \mathrm{nA}$ for the $1 \mathrm{~V}$ low- $V$, processes, respectively. Such a large impact on speed and standby power dissipation due to threshold variation in a low- $V$, scenario is intolerable and circuit techniques to address this problem are discussed briefiy here.

Multi-threshold voltage: A process with multiple threshold voltage transistors offers circuit design flexibility at the cost of additional threshold adjust masks and an implants. Low- $V$, transistors can be used for circuit speed and high- $V$, transistors can be used to reduce the leakage current [Shinichiro93].

Back-bias $V$, adjusting: Threshold voltage can be held constant by adjusting the substrate voltage. Figure 2.1 shows negative feedback circuitry which generates a substrate voltage to keep $V_{\text {tp }}$ equal to a constant $V_{\text {ref }}$ [Shoji92] [Bazarjani95d]. The amplifier in this circuit operates with supply voltages of $V_{P}$ and $V_{D D}$ where $V_{P}>V_{D D}$ is the positive supply (generated by a charge-pumped circuit) to keep source/drain and bulk junctions reverse biased at all times.

A self-substrate-biasing circuit technique is also reported in [Kobayashi94] to reduce the 


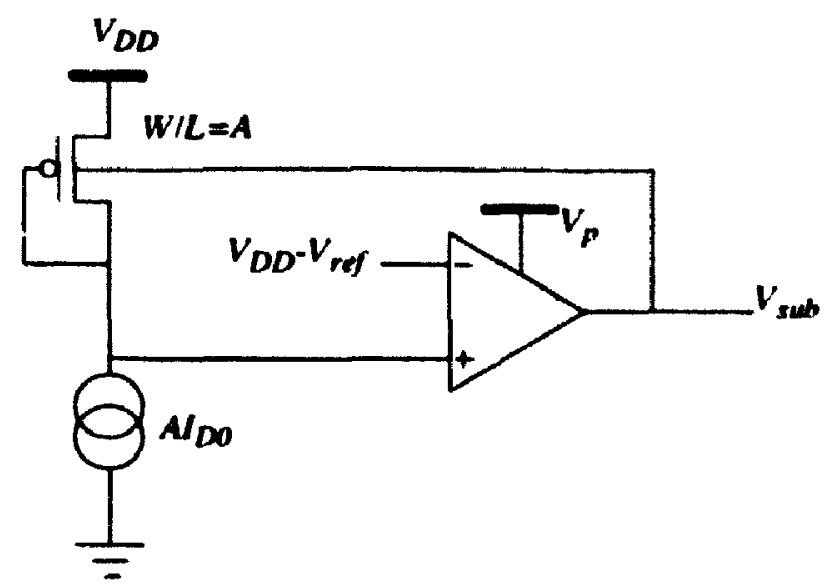

Figure 2.1: A negative feedback $V_{\text {, adjust circuit }}$

$V_{t}$ fluctuation. In [Kuroda96], a back-bias feedback circuit that adjusts $V$, to $0.1 V$ in active mode and $0.5 \mathrm{~V}$ in the standby mode is discussed. A complete study of $V$, adjusting circuits in SOI through back-bias along with the charge pump circuitry is under investigation [Soreefan96].

\section{Summary}

Supply and threshold voltages of future deep submicron CMOS technologies are being scaled down. Reducing the threshold voltage increases the off-current due to nonscalability of subthreshold swing, $S$, limiting the minimum $V_{f}$.

\subsection{Low-Voltage Mixed-Signal Design}

Low-voltage micropower circuit techniques were developed in the late 1960s and early 1970s for electronic wristwatches. These circuits had to operate from a single cell $1.35 \mathrm{~V}$ mercury or $1.5 \mathrm{~V}$ silver oxide battery. The operating frequencies of these circuits were typically a few $\mathrm{kHz}$ and their total power consumption was below $1 \mu \mathrm{W}$. Other lowpower applications include calculators, hearing aid devices, and pacemakers. In all these systems, the required speed performance of the circuit is low, typically less than $1 \mathrm{MHz}$.

The technology of choice for low-power applications has been CMOS due to its very low static power dissipation. Techniques to build low threshold voltage MOSFETs with $V,-0.5-0.8 \mathrm{~V}$ were developed in the late 1960s [Nagane69][Lewenberger69] and 
methods of adjusting the threshold voltage by doping implants were successfully demonstrated by Swanson [Swanson72].

In this section, limitations to low-voltage digital and analog designs due to circuit topology are discussed and some recent research in the area of low-voltage digital and analog circuits is presented. Finally, the impact of supply voltage scaling on power consumption of digital and analog circuits is considered.

\subsubsection{Limits on Digital CMOS Supply Voltage Scaling}

A lower limit on the supply voltage of digital circuits is set by the requirement that the voltage gain of a logic gate must be greater than unity. This condition is necessary to obtain regeneration of logic levels at the output of each gate.

In [Swanson72], MOSFET equations in weak inversion are derived and low-voltage operation of a CMOS inverter circuit is analyzed. Voltage gain of the CMOS inverter at mid-rail is found to be: $\left(V_{D D} / 2\right)(q /(n k T))$. Thus, the minimum required supply voltage for digital CMOS is

$$
V_{\left.D D\right|_{\min }}>\frac{2 n k T}{q}
$$

This is the theoretical lower bound for the supply voltage of a static CMOS inverter circuit. about 50-70 mV at room temperature. Some experimental circuits that have demonstrated the validity of the above limit include: a $100 \mathrm{mV}$ 11-stage ring oscillator [Swanson74], a 7-stage ring oscillator operating at $75 \mathrm{mV}$ at room temperature and $27 \mathrm{mV}$ at $77^{\circ} \mathrm{K}$ [Burr95].

The present supply voltage of $3.3 \mathrm{~V}$ for CMOS is a factor of 47 larger than the theoretical limit of $70 \mathrm{mV}$ found in (2-5). Therefore, a large margin for supply voltage reduction exists for CMOS.

In CMOS logic, a certain energy ( $\left.C V^{2} / 2\right)$ is stored on the gate capacitance to represent logic 1 . This stored energy must be greater than the thermal noise in that capacitor. The mean thermal noise energy in a capacitor is $k T / 2$ and in [Stein77], it is shown that for an error rate of $10^{-19}$ the minimum energy required per operation is $165 \mathrm{kT}$. Considering this energy requirement and the minimum supply voltage of (2-5), a logic operation 
involves only 118 electrons.

At this point, it would be interesting to see what are the minimum required supply voltage and logic swing for a bipolar circuit. A similar study for a Current Mode Logic (CML) gate. Figure 2.2, shows that exceeding unity gain requires the logic swing to be [Gray9.3]

$$
V_{\text {Swing }}>\frac{2 k T}{q}
$$

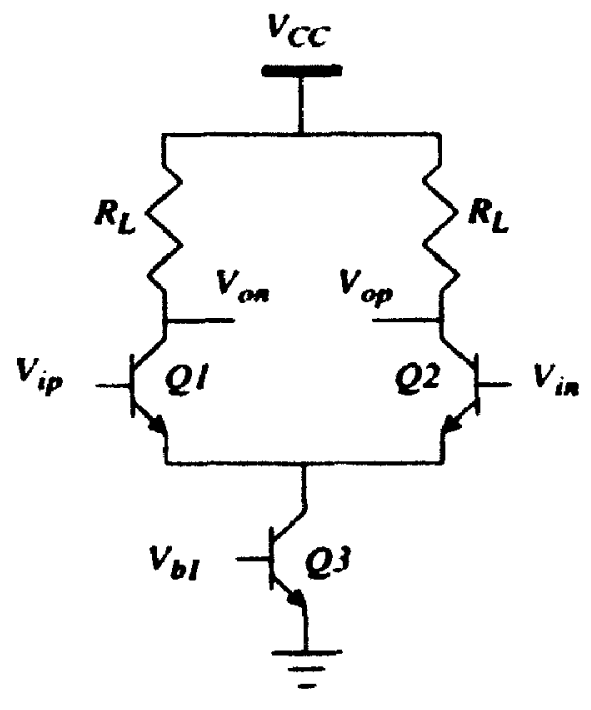

Figure 2.2: $A$ basic CML inverter-buffer gate

At rnom temperature, the lower bound of logic swing for bipolar circuits is about 5() $\mathrm{mV}$.

The lower limit of the power supply voltage for this CML gate is determined by the voltage required to turn on the input transistors $\mathbf{Q 1}$ and $\mathbf{Q} 2$ which is

$$
\left.V_{C C}\right|_{\min }=V_{B E}+V_{S w i n g}+V_{C E}
$$

Assuming a tum-on $V_{B E}$ of $0.75 \mathrm{~V}$. a $50 \mathrm{mV}$ logic swing, and a minimum $V_{C E}$ of $0.2 \mathrm{~V}$, the minimum required supply voltage for the CML gate is about $1 \mathrm{~V}$.

Existing $3 \mathrm{~V}$ CML gates, with a logic swing of $200 \mathrm{mV}$, operate close to the limits of $1 \mathrm{~V}$ power supply (higher by a factor of 3 ) and $50 \mathrm{mV}$ logic swing (higher by a factor of 4).

Thus, there is a very narrow margin for supply voltage reduction of bipolar CML gates. 


\subsubsection{Limits on Analog CMOS Supply Voltage Scaling}

This section is an investigation into the lower limit of the supply voltage for an opamp. analog switch, and current mirror-the three basic building blocks commonly used in analog SC and SI circuits.

\section{Operational Transconductance Amplifier}

Consider a simple single-stage fully differential opamp circuit, as shown in Figure 2.3. The minimum input common-mode voltage required for the proper operation of this

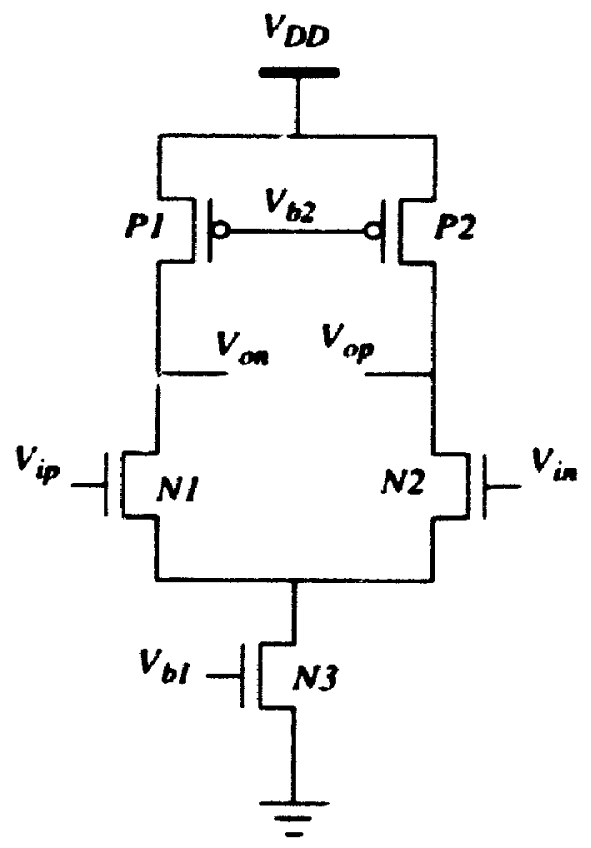

Figure 2.3: A fully differential CMOS Opamp

amplifier is determined by the voltage needed to turn on the input differential pair MOSFETs, transistors $\mathrm{N} 1$ and $\mathrm{N} 2$, as given by

$$
\left.V_{i c m}\right|_{\min }=V_{\text {In1 }}+V_{o n 1}+v_{D S 3}
$$

Here. $V_{i, m}$ is the input common-mode voltage, $V_{i n 1}$ is the threshold voltage of the transistor M1, $v_{D S 3}$ is the drain-source voltage of the transistor $\mathrm{N} 3$, and $v_{\text {on } 1}$ is the gate to source overdrive voltage of the transistor $\mathbf{N} 1$ which is defined as

$$
v_{\text {on }}=v_{G S}-V_{t}
$$


The minimum $v_{D S 3}$ required to keep the transistor $M 3$ in saturation is

$$
\left.v_{D S 3}\right|_{\min }=V_{\text {on } 3}=v_{G S 3}-V_{t 3} .
$$

If equal threshold voltage $V_{1}$ and overdrive voltage $V_{\text {on }}$ is assumed for all the transistors and also assumed that $V_{D D}>V_{i c m}$ (i.e., charge-pump techniques are not used). from equation (2-8) the lower bound on the supply voltage of the opamp is

$$
\left.V_{D D}\right|_{\text {min }}=V_{t}+2 V_{o n}
$$

The minimum required drain-source saturation voltage depends on the MOSFET moxte of operation. If the device is in weak inversion, the minimum saturation voltage required is about $(4-6) k T / q$ [Vittoz94]. which is 100 - $150 \mathrm{mV}$ at room temperature. However. the minimum drain-source saturation voltage of MOSFETs operating in strong inversion is about $200 \mathrm{mV}$.

In a typical $0.5 \mu \mathrm{m} C M O S$ process, with a threshold voltage of $0.6 \mathrm{~V}$. and assuming an overdrive voltage of $0.2 \mathrm{~V}$, the minimum supply voltage of this opamp is $1 \mathrm{~V}$. In this calculation, we ignored $V$, variations due to process fluctuation, temperature changes and back-bias voltage as well as constraints on input and output common-mode levels.

In a low- $V$, process, the minimum supply voltage of the opamp (Figure 2.3) may be determined by the drain-source saturation voltage (or $V_{o n}$ ) and the required output voltage swing $V_{\text {out (swing) }}$. Assuming equal gate to source overdrive voltage for all the transistors, the minimum supply voltage is

$$
V_{\left.D D\right|_{\min }}=3 V_{o n}+V_{o u r(\text { swing })}
$$

For $V_{\text {on }}=0.2 \mathrm{~V}$ and an output voltage swing of $0.4 \mathrm{~V}$, the minimum power supply voltage is $1 \mathrm{~V}$.

\section{Analog Switch}

An analog switch must have a full rail-to-rail signal handling capability with a low onresistance. An nMOSFET transistor used as a switch operates in thode mode with onresistance of approximately 


$$
R_{o n}=\frac{1}{\mu C_{o x} \frac{W}{L}\left(v_{G S}-V_{i n}\right)}
$$

A parallel nMOSFET and pMOSFET switch (transmission gate) has a rail-to-rail signal swing capability if the supply voltage is

$$
V_{D D}=V_{\text {in,e }}+V_{t p, e}+2 V_{o n}
$$

The effective threshold voltages. $V_{i n, e}$ and $V_{i p, e}$ are given by

$$
V_{t e}=V_{t 0}(M a x)+\Delta V_{t}(T)+\Delta V_{t}\left(v_{s B}\right)
$$

$V_{,}(\operatorname{Max})$ is the $3 \sigma$ upper value of threshold voltage due to process variations, $\Delta V_{t}(T)$ and $\Delta V_{,}\left(v_{S B}\right)$ are the threshold voltage changes due to temperature, and the back bias respectively and are given by

$$
\begin{gathered}
\Delta V_{t}(T)=a(25-T) \\
\Delta V_{t}\left(v_{S B}\right)=\gamma\left(\sqrt{2 \Phi_{B}+v_{S B}}-\sqrt{2 \Phi_{B}}\right) .
\end{gathered}
$$

Here, $T$ is temperature in degrees Celsius, and " $a$ " is the slope of threshold voltage as a function of temperature, which is about $1-2 \mathrm{mV} /{ }^{\circ} \mathrm{C}$. Other parameters are the body effect coefficient $\gamma=0.5$. the bulk potential $\Phi_{B}$, and the source to bulk voltage $v_{S B}$.

The on-conductance of a transmission gate switch as a function of signal level is shown in Figure $\mathbf{2 . 4}$ for three different values of power supply voltages [Vittoz93]. In this figure the

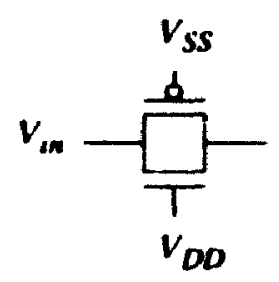

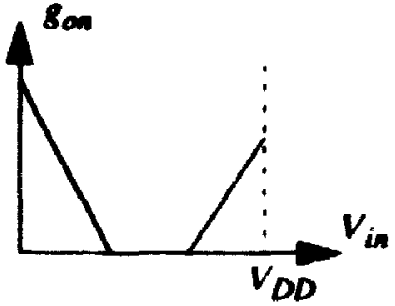

(a)

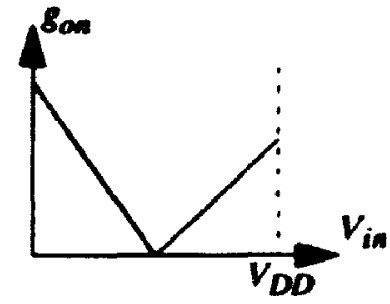

(b)

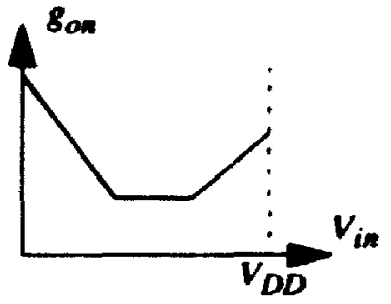

(c)

Figure 2.4: Complementary transmission gate switch on-conductance for (a) $V_{D D}<2 V_{1}$ (b) $V_{D D}=2 V_{1}$, and (c) $V_{D D}>2 V_{1}$ 
threshold voltages of nMOSFET and pMOSFET are assumed to be equal to $r$,

In a $0.5 \mu \mathrm{m}$ process with effective threshold voltages of $0.8 \mathrm{~V}$. a supply voltage of $2 \mathrm{~V}$ is required to turn the transmission gate on with a minimum overdrive of $11.2 \mathrm{~V}$ at mid-rail.

\section{Current mirror}

A current mirror circuit is a current gain cell that provides a weighted output copy of an input current. This circuit forms the foundation of SI signal processing and is also widely used to provide bias currents in such blocks as opamps and comparators. A simple current mirror circuit is shown in Figure 2.5. The voltage $V_{B}$ generates a current $I_{B}$ in the transistor $P$ which is fed to the diode-connected transistor $N 1$. Since transistors $N 2$ and NI have the same gate and source potential, their drain-source currents are related by

$$
\frac{I_{2}}{I_{1}}=\frac{(W / L)_{N 2}}{(W / L)_{N 1}}
$$

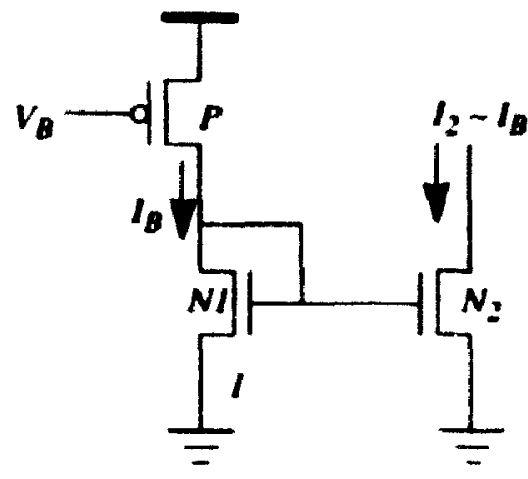

Figure 2.5: A simple current mirror cell

If $N 1$ and $N 2$ are identical transistors, $I_{2}$ will mirror $I_{B}$. For accurate operation. all the transistors in the circuit must be in the saturation regime. Transistor $N 1$ is dioxte connected and when it is turned on it will be in saturation with a gate (or drain) to source voltage of $V_{t}+V_{o n}$. Assuming the same overdrive voltage $\left(V_{o n}\right)$ for all the MOSFETs. transistor $\boldsymbol{P}$ requires a drain to source voltage of at least $V_{\text {un }}$ to be in saturation. Thus, the minimum required supply voltage for proper operation of the simple current mirror is

$$
V_{D D}>V_{l}+2 V_{o n}
$$


which is identical to the minimum supply voltage expression obtained for a simple opamp (2-11). Comparison of (2-11), (2-19) and (2-14) shows that switches are the bottleneck for low-voltage SC and SI operation.

\subsubsection{Previous Work on Low-Voltage Digital Circuits}

A low-voltage digital design using standard CMOS technology, by $\mathrm{A}$. Chandrakasan at Berkeley University, is described first. Then, a theoretical analysis by Dake Liu at Linkoping University, showing power savings achieved by supply voltage and threshold voltage down-scaling, is discussed. Finally, an experimental encoder-decoder system operating at $2(x) \mathrm{mV}$. by Jim Burr at Stanford University, is presented.

\section{Berkeley}

Power optimization at different levels of technology. circuit style, architecture, and supply voltag: xialing is considered in [Chandrakasan92]. The most efficient logic family was found to be CPL (Complementary Pass-transistor Logic) if low threshold nMOSFETs are available. Logic gates must be designed for the lowest acceptable speed to save unnecessary energy loss.

In a $2 \mu \mathrm{m}$ CMOS to:hnology, a power supply of $1.5 \mathrm{~V}$ is found to be the optimum for a large variety of eases. At such a low voltage $\left(V_{D D}=2 V_{t}\right)$. circuit speed may become unacceptably low for some applications. In such cases, techniques such as parallel architecture and pipelining can be used to obtain lower latency and higher throughput rates.

\section{Linkoping}

An extensive study of the impacts of supply voltage and threshold voltage scaling is reported in [Liuy3]. Reducing the supply voltage decreases the power consumption in CMOS logic $\left(P \propto f C V^{2}\right)$. However, speed performance $T \propto\left(V_{D D}\right) /\left(V_{D D}-V_{t}\right)^{2}$ is degraded unless the threshold voltage is scaled down.

In a $0.25 \mu \mathrm{m}$ CMOS technology it is shown that a factor of 40 reduction in power dissipation is possible. without any speed loss, by reducing the supply voltage from $3 \mathrm{~V}$ to $0.48 \mathrm{~V}$ and properly optimizing the MOSFETs threshold voltages from $0.7 \mathrm{~V}$ to $v_{\text {in }}=0.1 \mathrm{~V}$ and $v_{t p}=-0.01 \mathrm{~V}$. 


\section{Stanford}

Low-power research at Stanford University [Burryl] focuses on the use of near zero threshold voltage $(\sim 100) \mathrm{mV}$, MOSFETs and selecting a power supply voltage of $V_{D D}=3 V_{1}$. The threshoid voltage is tine-tuned by applying a back-hias prtential.

Low threshold voltage devices allow reduced power supply voltage. which in turn decreases AC power dissipation quadratically. However, DC power consumption, due (1) the subthreshold leakage, increases exponentially. The minimum total power dissipatton is obtained at a supply voltage where the AC component of the power dissipation and the DC power dissipation are equal. In a $2 \mu \mathrm{m}$ CMOS technology. $2(x) m \mathrm{~V}$ is show to to the optimum supply voltage for digital circuits with a logic depth of 10 and an activity ratio of 0.1. An experimental CMOS Encoder/Decoder circuit is demonstrated in [Burry4] to operate at $290 \mathrm{mV}$ supply voltage.

\subsubsection{Previous Work on Low-Voltage Analog Circuits}

Work on low-voltage analog circuits started in bipolar technology in the $1971 \mathrm{ls}$. Some reported IV circuits in bipolar technology include: a bandgap voltage reference [Vittoz77], operational amplifiers [Widlar78] [Huijsing85], an active lowpass tilter [Tanimoto91]. and an analog current mode multiplier cell [Chan95].

Low-voltage analog circuits in CMOS (the focus of this work) started in the late 1970, [Vittoz77] and early 1980s [Vittoz80] |Krummen\&2|. Low-voltage analog circuits are implemented using Switched-Capacitor (SC), active-RC, and Switched-Current (SI) tecnniques. This section starts by describing a I V $\Sigma \Delta A D$ converter using an active-RC technique. A $1.2 \mathrm{~V} \Sigma \Delta$ converter implemented with the SI technique is then dixcussed. Finally, three papers dealing with low-voltage SC filters are presented.

\subsubsection{Low-Voltage Active-RC Circuits}

A $1 \mathrm{~V}$ second-order $\Sigma \Delta$ modulator, implemented in a $0.5 \mu \mathrm{m}$ multi-threshold voltage CMOS technology, is reported in [Matsuya94]. Integrators in the $\Sigma \Delta$ modulator are implemented using an active-RC technique, as shown in Figure 2.6. For an oversampling ratio of 16, this modulator has a SNDR of $51 \mathrm{~dB}$ and a dynamic range of $58 \mathrm{~dB}$. The modulator operates at a clock frequency of $6.14 \mathrm{MHz}$ (signal bandwidth of $192 \mathrm{kHz}$ ) 
and consumes $1.56 \mathrm{~mW}$ at a $1 \mathrm{~V}$ power supply voltage.

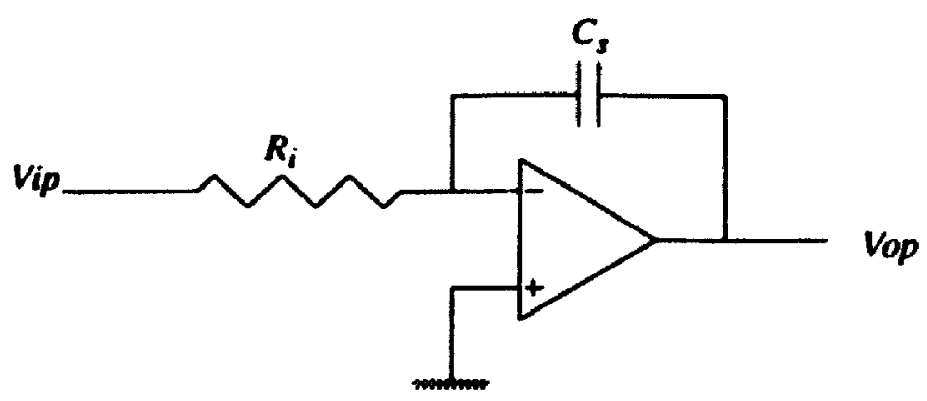

Figure 2.6: An active-RC integrator

Since subthreshold leakage current through low- $V$ analog switches was the main source of concern in this design, SC implementation was ruled out. In Chapter 3 of this thesis, the impact of leakage current through analog switches on the accuracy of SC circuits are discussed and methods of reducing the subthreshold leakage are also presented.

A drawback of integrated active-RC circuits is inaccuracy in the RC time constant, because monolithic resistors and capacitors have a tolerance of more than $\pm 15 \%$ and do not track each other. For lowpass $\Sigma \Delta$ modulators, variations in the RC time constant causes a gain variation and is not critical.

\subsubsection{Low-Voltage Switched-Current Circuits}

Recently [Tan95], a low-voltage second-order SI $\mathbf{\Sigma}$ modulator was reported which operates at $1.2 \mathrm{~V}$. The circuit, however, is not a true $1.2 \mathrm{~V}$ part because the switches operate from a higher extemal supply voltage.

The circuit is implemented in a $0.8 \mu \mathrm{m}$ digital CMOS process using a fully differential SI memory cell with a common-mode feed forward circuit. This modulator is clocked at $1 \mathrm{MHz}$ and consumes $0.78 \mathrm{~mW}$. The measured SNDR and SNR for an oversampling ratio of 64 are $55 \mathrm{~dB}$ and $51 \mathrm{~dB}$ respectively. Ideally, this modulator should achieve a SNDR of more than $80 \mathrm{~dB}$. The low performance of this SI circuit is attributed (by the authors of the original paper) to circuit noise generated by the first current copier cell.

A review of SI lechnique is provided in section 2.5. In section 2.6 it will be shown that SC has some advantages over SI for low-voltage applications. 


\subsubsection{Low-Voltage Switched-Capacitor Circuits}

In CMOS, SC is the dominant technique for implementing analog circuits due to its high circuit accuracy and low distortion even at low supply voltages [Castello91]. For lowvoltage applications, the most critical component in a SC circuit (and a SI circuit) is the transmission gate switch. which requires a gate voltage of at least $2 v$, for proper full swing signal handling. In SC circuits, few switches usually require rail-to-rail signal swing. For instance, in the non-inverting SC integrator of Figure 2.7 only the input switch (S1) needs to be implemented with a transmission gate, an nMOSFET and an pMOSFET in parallel. All the other switches are connected to analog ground $V_{a g}=V_{D D} / 2$ and thus can simply be nMOSFET transistors.
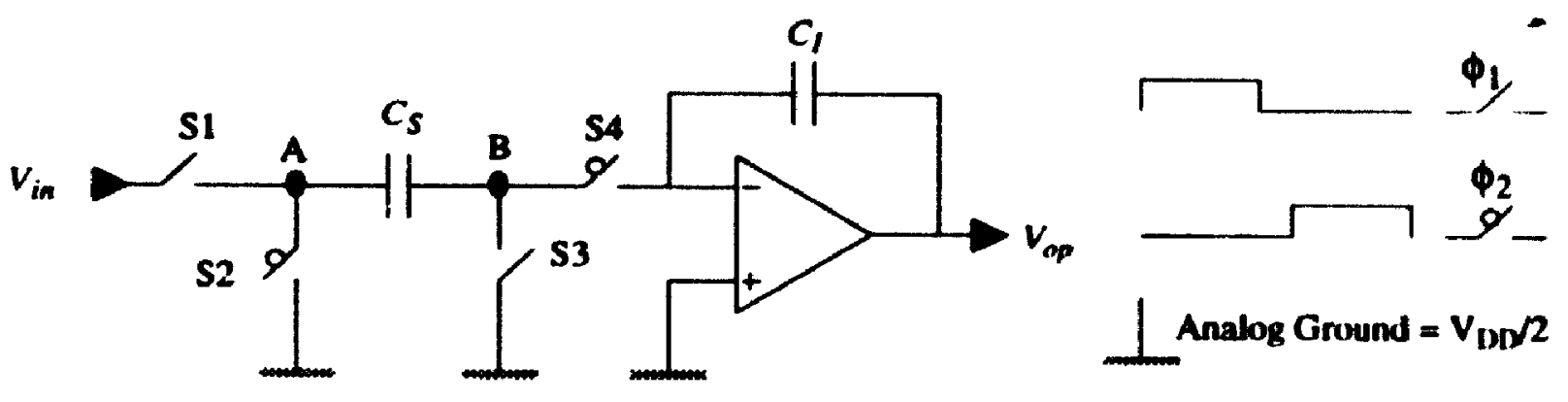

Figure 2.7: A non-inverting SC integrator and its associated 2-phase non-overlapping clock

In general, to achieve full signal transmission through a MOSFET switch with acceptable on-resistance, either the gate voltage must be increased (e.g., clock voltage multiplication) or the threshold voltage of MOSFETs must be reduced. A third solution would be to eliminate the need for switch $S 1$. In the following section, the above methods of lowvoltage SC design are described.

\section{(I) Clock Voltage Multiplication}

If the supply voltage is less than $2 V_{t}$, the MOSFET switches will not be able to conduct when biased at the mid-rail. Clock voltage boosting is an effective way of increasing the conductance of the MOSFET switches. This is the most commonly used technique in lowvoltage SC circuits [Krummen83] [Callias89] [Castello91] [Wayne92] [Grilo96] [Au96].

A biquadratic SC lowpass filter operating at $1.5 \mathrm{~V}$ is reported in [Castello91] for 
telephony applications. This filter uses a fully differential architecture and is fabricated in a $0.8 \mu \mathrm{m}$ BiCMOS technology. The clock frequency is $447 \mathrm{kHz}$ and the measured THD is $-70 \mathrm{~dB}$ for a $1.5 \mathrm{Vpp}$ signal.

This technique requires extra circuitry for voltage multiplication, may need an off-chip capacitor, and is noisy. Another drawback of voltage boosting is that in deep submicron modern processes, the higher electric field of clock voltage is a reliability hazard and is not acceptable.

\section{(II) Low-V,MOSFETs}

Reducing the threshold voltage of MOSFETs will reduce the on-resistance of the switches. In [Adachi90], a lower threshold voltage nMOSFET $\left(V_{t}=0.2 \mathrm{~V}\right)$ is fabricated by adding an extra mask for threshold adjust implant to a double-poly $2 \mu \mathrm{m}$ CMOS process. A 7thorder Chebyshev SC lowpass telephony filter, implemented in this process, operates at $1.4 \mathrm{~V}$ and consumes $0.49 \mathrm{~mW}$. This filter is clocked at $111 \mathrm{kHz}$ and has a cutoff frequency of $3 \mathrm{kHz}$. The in-band noise for an input signal with a $1 \mathrm{Vpp}$ signal is measured to be $-67 \mathrm{dBm}$. This performance is comparable to the SC filters operating at $5 \mathrm{~V}$.

This method may require process modification. However, in multi-threshold processes [Sun92] [Mutoh93], low- $V$, MOSFETs are available at no extra cost. A low-cost method

of fabricating low- $V$, devices in a dual poly gate process is also discussed in Chapter 3. An advantage of this approach (i.e., the low- $V$, MOSFET switch) is compatibility with the future of CMOS technology and low-power digital design [Liu93][Burr94] because power supply scaling is forcing $V_{\ell}$ down-scaling.

(III) Switched-Opamp Circuits

In a SC circuit most switches are connected to analog (or virtual) ground. By selecting an analog ground which is very close to $V_{S S}$, nMOSFETs that conduct well can be used as switches. The input switch that requires full signal swing, switch $S I$ in Figure 2.7, can be replaced with a switched-opamp as shown in Figure 2.8 .

A second-order lowpass filter implemented using a switched-opamp is described in [Crols94] and operates at $1.5 \mathrm{~V}$ while consuming $110 \mu \mathrm{W}$. This biquadratic filter is 


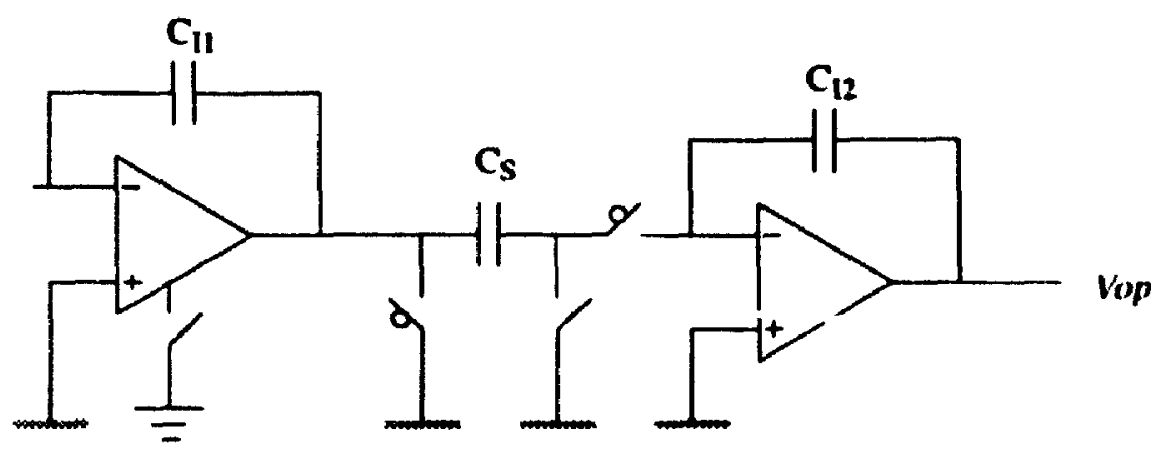

Figure 2.8: A switched-opamp SC integrator

clocked at $115 \mathrm{kHz}$ and has a cutoff frequency of $1.5 \mathrm{kHz}$. For an output swing of $0.55 \mathrm{Vpp}$, the total harmonic distortion is measured to be $-64 \mathrm{~dB}$. The dynamic range of this filter is about $69 \mathrm{~dB}$.

In a SC circuit, the very first input switch is not driven by an integrator and therefore can not be replaced with a switched-opamp. One solution is to implement the first stage of the circuit using an active-RC technique. This causes a gain error, which may be acceptable. while all the loop time constants are determined by SC topologies.

\subsubsection{Impact of Voltage Scaling on Power Dissipation of Mixed-Signal Circuits}

Power dissipation in a circuit must be studied in relation to other performance parameters of the circuit. For instance, in digital circuits there is a trade-off between power dissipation and speed of the circuit. In general. one is interested in reducing power consumption while not sacrificing speed. This means reducing the energy consumption of the circuit. In analog circuits, power minimization must be considered along with the speed and dynamic range of the circuit. In this section, the effects of supply voltage scaling on energy dissipation of digital circuits and power dissipation of analog circuits (for a tixed speed and dynamic range) are investigated.

\section{Power Dissipation in Digital CMOS}

In general, power dissipation is caused by two types of current: a dynamic current and a static current. A CMOS inverter, Figure 2.9, is used to illustrate various components of the power dissipation in static CMOS logic.

The static power dissipation is caused by MOSFET subthreshold leakage and leakage 


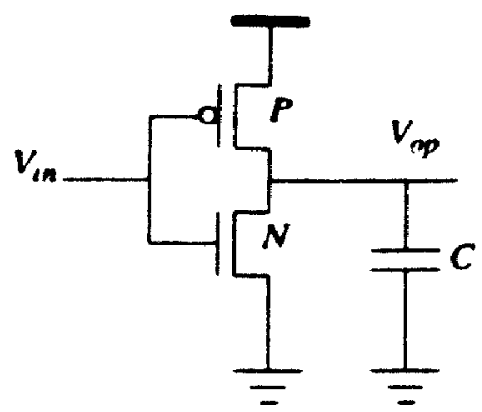

Figure 2.9: CMOS inverter

currents through reverse-biased junctions $\left(I_{\text {leak }}\right)$. When switching, charging and discharging of the load capacitor $(C)$ is the main source of power dissipation which is quadratically proportional to the supply voltage. During each input transition, there is also a short circuit (crowbar) current $\left(I_{s c}\right)$ flowing directly from the power supply to ground when both nMOSFET and pMOSFET are temporarily on $\left(V_{\text {in }}<V_{i n}<V_{D D}-V_{i p}\right)$. The total power consumption is

$$
P=I_{\text {leak }} V_{D D}+f C V_{D D}^{2}+I_{S C} V_{D D}
$$

Dynamic power dissipation $f C V_{D D}^{2}$ is usually the dominant term in (2-20) [Burr 91 ]. Energy dissipation is a measure of the power-delay product, which is

$$
E=C V_{D D}^{2}
$$

Supply voltage scaling reduces the energy dissipation in digital CMOS circuits quadratically.

\section{Power Dissipation in Analog SC Circuits}

The SC integrator shown in Figure 2.7 is considered for the study of power dissipation in analog $\mathrm{SC}$ circuits.

Just as for digital circuits, there are two types of power dissipation: dynamic and static. Leakage currents, DC bias currents in bias circuitry, and opamp bias currents for class A operation will all contribute to static power dissipation.

$$
P_{\text {static }}=I_{\text {leak }} V_{D D}+\left(I_{\text {bias }}+I_{A}\right) V_{D D}
$$


Dynamic power is dissipated by the clock generator. charging and discharging MOSFET switches, as well as by amplitiers charging load capacitors $\left(C_{S}\right.$ and $\left.C_{l}\right)$. The dynamic power dissipated in clock drivers due to the gate capacitance of MOSFET switches is

$$
P_{s w^{\prime}}=f_{\text {clock }} C_{s w} \cdot \stackrel{2}{n D} .
$$

The average power dissipated in driving the load capacitors is given by the following equation [Castello85]:

$$
P_{C}=\frac{2}{\pi} V_{i} V_{D D} C_{s} f_{\text {ilock }}
$$

Here, $V_{i}$ is the peak amplitude of a sinusoidal signal and $f_{\text {clock }}$ is the clock frequency. In analog circuits an important figure of merit is dynamic range (DR), which is a measure of circuit accuracy and is given by

$$
D R=\frac{\text { Signal }(r m s)}{\text { noise }}=\frac{v_{i, r m s}}{\sqrt{k T / C_{s}}}
$$

$V_{i, r m s}$ is the RMS value of the input sinusoidal signal, $k=1.38 \times 10^{-2.3}(\mathrm{~J} / \mathrm{K})$ is the Boltzman constant, and $T$ is the temperature in degrees Kelvin. If we assume that the input is a sinusoidal signal and can swing between supply rails, the RMS value of the input is

$$
V_{i, r m s}=\frac{V_{D D}}{2 \sqrt{2}} .
$$

Equations (2-24), (2-25), and (2-26) can be combined to describe power dissipation in the sampling capacitor as a function of dynamic range and clock frequency. The resulting equation is

$$
P_{C}=\frac{D R^{2}}{2 \pi}(k T) f_{\text {clock }}
$$

This equation shows that for a given dynamic range the power dissipated in driving the load capacitor is independent of the power supply voltage. 
Thus, in the limit, when DC power dissipation and power dissipated due to the switches are negligible compared to $\mathrm{AC}$ power dissipation in driving the load capacitors, reducing the power supply will not change the power dissipation in the analog circuitry.

In the above analysis, limitation on the unity gain bandwidth $\left(\omega_{N}\right)$ was not included. $A$ more realistic approach is to relate power dissipation to $D R$ and $\omega_{\mu}$. Power dissipation in driving the load capacitor is

$$
P=V_{D D} I
$$

The speed of a SC circuit is determined by the unity gain bandwidth of the opamp which is

$$
\omega_{u}=\frac{g_{m}}{C}
$$

Here, $g_{m}$ is the transconductance of the opamp which has the following relation with the quiescent current:

$$
I=g_{m} \frac{V_{o n}}{2}
$$

Combining equations (2-25), (2-26), (2-28), (2-29) and (2-30), the power dissipation can be expressed in terms of dynamic range, speed, and supply voltage as

$$
P \propto D R^{2} k T \omega_{u}\left(\frac{V_{o n}}{V_{D D}}\right)
$$

Thus, reducing the supply voltage, if accompanied by an increase in load capacitance to compensate for dynamic range loss, will increase the total power dissipation in analog circuits. Scaling $V_{\text {on }}$ with the supply voltage would give constant power dissipation but would require process scaling to correct for the reduced transistor $f_{t}$, which is inversely proportional to channel length squared, $f_{t} \propto V_{o n} / L^{2}$.

If supply voltage scaling does not require increasing the load capacitor (and consequently increasing the quiescent current), power dissipation will be reduced linearly with supply voltage down-scaling. This happens when the value of the capacitor is determined by other considerations such as stability or matching, and is higher than the required value 
due to $k T / C$ noise.

\section{Summary}

Limits to low-voltage digital CMOS circuits indicate that there is a large margin for supply voltage down scaling. A review of the literature in the area of low-voltage circuits suggests that digital circuits using low- $V$, MOSFETs achieve a great saving in power dissipation. In analog SC circuits, if the process is fixed lowering the supply voltage will increase the power dissipation in driving the load capacitance. On the other hand. technology scaling allows $V_{\text {on }}$ to be scaled down along with the power supply without sacrificing device $f_{l}$. In this case, power dissipation for a given dynamic range and unity gain bandwidth will remain unchanged.

\subsection{Oversampled Sigma-Delta Modulator Overview}

Oversampled $\Sigma \Delta$ modulation is a noise shaping technique that converts an analog input signal into a simple digital code (typically l-bit) at a sampling frequency which is much higher than the Nyquist rate. A block diagram of a general $\mathbf{n}$-bit $\mathbf{\Sigma} \Delta$ modulator comprising a loop filter, an n-bit $A / D$, and an n-bit D/A in a negative feedback loop is shown in Figure 2.10.

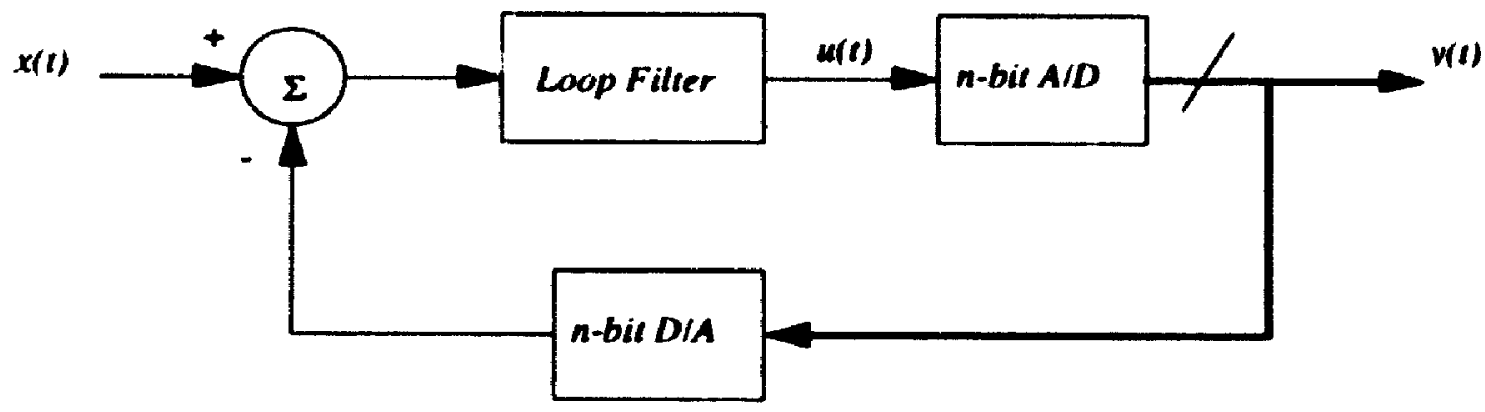

Figure 2.10: A block diagram of a $\Sigma \Delta$ modulator

The loop filter shapes the quantization noise out of the band of interest. If a lowpass filter is placed in the loop, the quantization noise will be highpass filtered and if a bandpass filter is chosen as loop filter, the quantization noise will be band-reject filtered. In a sigmadelta $A / D$ converter, the out of band quantization noise is removed by a digital filter that also performs decimation. 
$\Sigma \Delta$ is becoming the method of choice for high-precision low- or medium-speed AVD converters. This is because compared to other techniques, such as flash and pipelined A/D. the analog circuitry in a $\Sigma \Delta$ modulator is simple and relatively insensitive to circuit imperfection and component mismatch. Also, due to the oversampling, the antialiasing filter at the input of a $\Sigma \triangle A / D$ converter has relaxed requirements compared to that of a Nyquist rate $\mathbf{A} / \mathrm{D}$ converter. However, $\Sigma \Delta$ modulators require a large digital signal processing unit for the decimation filter. Both simplicity of analog circuits and extensive use of digital circuits in $\Sigma \Delta$ modulators are highly compatible with scaled-down CMOS technologies.

In Figure 2.10, if the transfer function of the loop filter is denoted as $H(z)$ and the n-bit A/D (multilevel quantizer) is modeled as an additive noise $Q(z)$, the equivalent linear feedback system of Figure 2.11 is obtained.

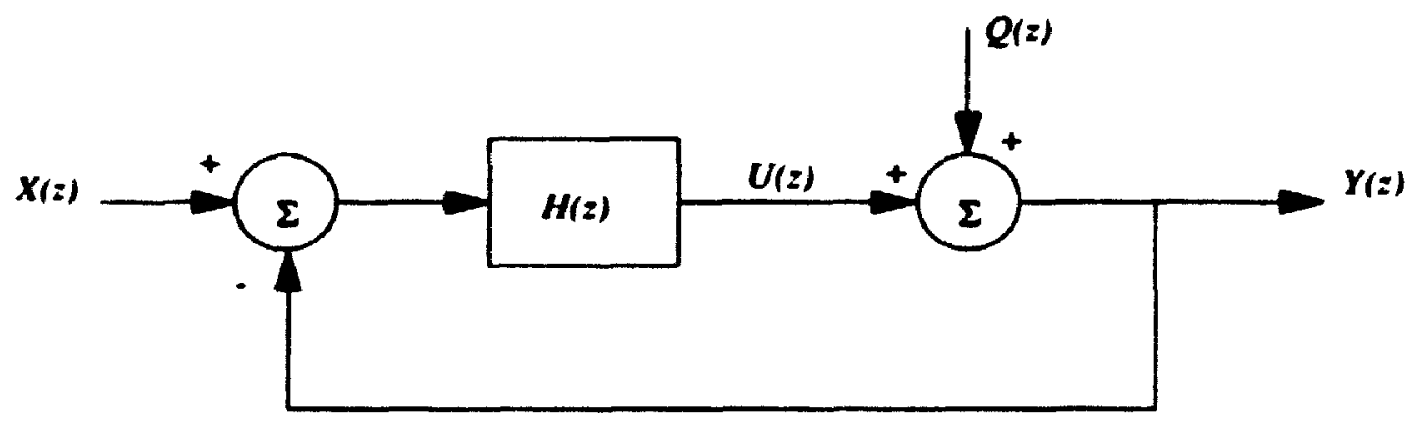

Figure 2.11: Equivalent linear system of Figure 2.in

The expression for the output $z$-transform in terms of the signal transfer function $F_{X}(z)$ and noise transfer function $F_{Q}(z)$ is

$$
Y(z)=F_{X}(z) X(z)+F_{Q}(z) Q(z) .
$$

The signal transfer function and the noise transfer function are respecively

$$
\begin{aligned}
& F_{X}(z)=\frac{H(z)}{1+H(z)} \\
& F_{Q}(z)=\frac{1}{1+H(z)}
\end{aligned}
$$




\subsubsection{Lowpass Sigma-Delta Modulators}

A simple tirst-order $\Sigma \Delta$ modulator is obtained by letting the loop filter be a discrete-time integrator and also allowing the quantizer to have only two levels of $\pm \Delta / 2$. The z-domain transfer function of a unity delay integrator is

$$
H(z)=\frac{z^{-1}}{1-z^{-1}}
$$

Substituting the above $H(z)$ into equations (2-33) and (2-34) will give the corresponding signal transfer function and noise transfer function, as follows:

$$
\begin{gathered}
F_{X}(z)=z^{-1} \\
F_{Q}(z)=1-z^{-1} .
\end{gathered}
$$

The signal transfer function is simply a unit delay and the noise transfer function is a tirstorder differentiator which behaves like a highpass filter. Since $F_{Q}$ has a zero at DC, the quantization noise is reduced at low frequencies and is pushed to high frequencies.

If the quantization error is assumed to be white noise with a mean square value of $\Delta^{2} / 12$. the quantization noise in the signal band $0<f<f_{B}$ is approximately [Candy 92 ]

$$
n_{0}^{2}=\frac{\Delta^{2}}{12} \frac{\pi^{2}}{3}\left(\frac{1}{O S R}\right)^{3}
$$

where $O S R$ is the oversampling ratio defined as the ratio of the sampling frequency $f_{s}$ to the Nyquist rate of the signal $2 f_{B}$.

Increasing the $O S R$ by a factor of 2 reduces the quantization noise, in the first-order $\Sigma \Delta$ modulator, by $9 \mathrm{~dB}$ which adds 1.5 bits to the resolution.

A second-order noise shaping is achieved by allowing a double differentiator noise transfer function as defined by

$$
F_{Q}(z)=\left(1-z^{-1}\right)^{2}
$$

This noise transfer function has a double zero at DC. The corresponding single loop filter 
transfer function is obtained by substituting (2-39) into (2-34) and doing some simple algebra. The second-order $\Sigma \Delta$ modulator loop tilter transfer function is

$$
H(z)=\frac{z^{-1}\left(2-z^{-1}\right)}{\left(1-z^{-1}\right)^{2}} .
$$

The in-band power of quantization noise at the output of a second-order $\Sigma \Delta$ modulator is [Candy92]

$$
n_{0}^{2}=\frac{\Delta^{2}}{12} \frac{\pi^{4}}{5}\left(\frac{1}{O S R}\right)^{5}
$$

Doubling the $O S R$ reduces the quantization noise by $15 \mathrm{~dB}$, adding 2.5 bits to the resolution of a second-order $\Sigma \Delta$ modulator.

\subsubsection{Bandpass Sigma-Delta Modulators}

The basic principle of noise shaping can be extended by placing the quantization noise nulls at an arbitrary center frequency $f_{0}$. Then, the quantization noise will be pushed away from the signal band $(B W)$ at the desired center frequency.

A simple way of designing bandpass $\mathbf{\Sigma} \Delta$ modulators is to perform a lowpass to bandpass transformation. One such transformation in the discrete-time domain is achieved by the following change of variable,

$$
z^{-1} \rightarrow-z^{-2}
$$

This transformation maps the zeros of the lowpass prototype from $D C$ to $\pm f_{s} / 4$. Therefore, noise in the resulting bandpass modulator is suppressed around the $f_{s} / 4$ and the $3 f_{s} / 4$ frequencies. The stability and SNR characteristics of this bandpass modulator will be identical to that of the lowpass prototype [Jantzi].

In the following, the above transformation, equation (2-42), is applied to a first-order lowpass $\Sigma \Delta$ modulator $H_{l p 1}$. The resulting bandpass modulator, $H_{b p 2}$, is a second-order modulator as expected from (2-42). 


$$
H_{l p 1}=\frac{-z^{-1}}{1-z^{-1}} \rightarrow H_{b p 2}=\frac{z^{-2}}{1+z^{-2}}
$$

The above mapping is illustrated in Figure 2.12.

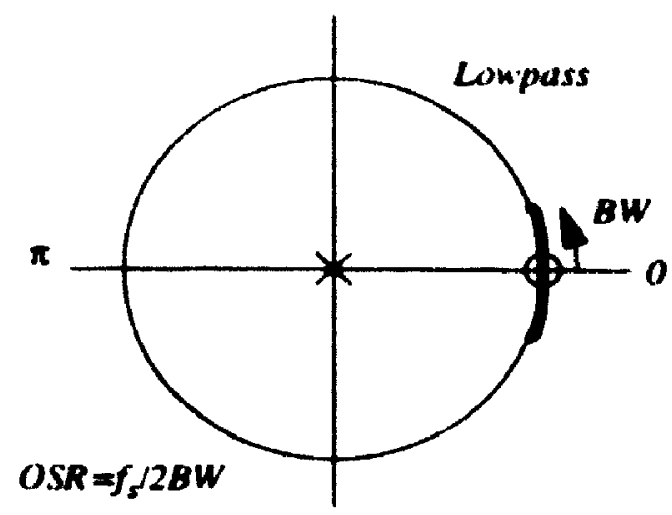

(a)

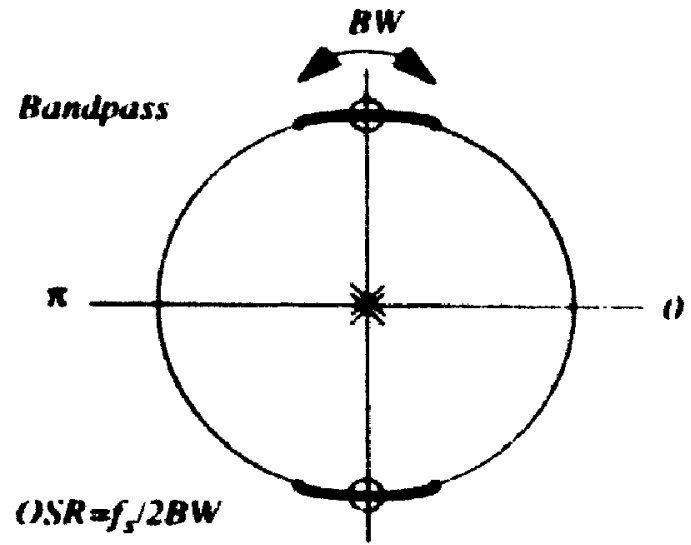

(b)

Figure 2.12: Pole/zero placement of (a) a tirst-order lowpass $\Sigma \Delta$ and (b) a secondorder bandpass $\Sigma \Delta$ derived by $z^{-1} \rightarrow-z^{-2}$ uransformation

For a bandpass $\Sigma \Delta$ modulator with center frequency $f_{0}$ located at $f_{s} / 4$, the oversampling ratio is simply $2 f_{0} / B W$.

The second-order $\Sigma \Delta$ modulator represented by $(2-40)$ can also be transfomed to a fourthorder bandpass $\Sigma \Delta$ modulator as follows:

$$
H_{l p 2}(z)=\frac{-z^{-1}\left(2-z^{-1}\right)}{\left(1-z^{-1}\right)^{2}} \rightarrow H_{b p 4}(z)=\frac{z^{-2}\left(2+z^{-2}\right)}{\left(1+z^{-2}\right)^{2}}
$$

The stability of this fourth-order bandpass $\Sigma \Delta$ modulator $H_{b p 4}$ is assured due to the stability of the second-order lowpass prototype $H_{l p 2}$.

\subsubsection{High-Speed SC Bandpass Sigma-Delta Modulator}

Bandpass $\Sigma \Delta$ modulators allow AD conversion to be performed on narrow-band signals at IF (Intermediate Frequency) frequencies. These ADs are useful in some communication systems, such as AM radio, digital radio, and in high speed modems. Digitizing the analog signal at a high IF and processing the signal in the digital domain is 
desirable due to the robustness of digital circuits.

High-speed analog signal processing will be dominated by continuous-time Gm-C [Shoaei94] or LC based circuits. These circuits typically require tuning circuitry which is non-trivial. Recently, a high-speed bipolar sampled-data bandpass $\Sigma \Delta$ modulator has been reported [Varelas 96 ]. Here, we focus on high-speed SC implementation of bandpass $\Sigma \Delta$ modulator using standand CMOS processes.

In the following, monolithic bandpass $\mathbf{\Sigma} \Delta$ modulators reported in refereed publications are discussed and the ir performances summarized.

The first reported monolithic bandpass $\mathrm{IA}$ modulator was a fourth-order SC circuit with optimally placed zeros for noise transfer function [Jantzi92]. This modulator was implemented in a $3 \mathrm{\mu m}$, $\pm 5 \mathrm{~V}$, double-poly analog CMOS process. It operated at a $1.82 \mathrm{MHz}$ clock and achieved a $63 \mathrm{~dB}$ SNDR for signals at $455 \mathrm{kHz}$ IF with a $10 \mathrm{kHz}$ bandwidth.

Another fourth-order SC bandpass $\mathrm{EA}$ modulator operating at a $7.2 \mathrm{MHz}$ clock freque ' y was reported in [Longo93]. This modulator has a transfer function similar to $H_{b p 4}$ in equation (2-44) and is implemented in a 1 $\mathrm{mm}$ double-poly CMOS technology. The resonators are built using two SC delay cells in a negative feedback loop. Over a $30 \mathrm{kHz}$ bandwidth centered at $1.8 \mathrm{MHz}$. the measured SNDR is $75 \mathrm{~dB}$. This modulator operates at $5 Y$ and consumes $40 \mathrm{~mW}$.

A high-speed second-onder SC $\Sigma \Delta$ modulator operating at a clock frequency of $42.8 \mathrm{MHz}$ was described by Singor and Snelgrove in [Singor94]. This modulator was impiemented in a $0.8 \mu \mathrm{m}$, ible-poly BiCMOS technology. The transfer function of this modulator is identical to $H_{b p^{\prime}}$ in equation (2-43) and the resonator is implemented using LDI and FE SC integrators. A performance of $55 \mathrm{~dB}$ SNDR was achieved in a $200 \mathrm{kHz}$ bandwidth centered at $10.7 \mathrm{MHz}$, while dissipating $60 \mathrm{~mW}$ from a $5 \mathrm{~V}$ power supply.

In [Song95), a hardware efficient fourth-order bandpass SC $\Sigma \Delta$ is described which requires two opamps instead of four. The two opamps consume $0.8 \mathrm{~mW}$ from a $3.3 \mathrm{~V}$ power supply. This modulator is based on a 2-path system and is designed in a $2 \mu \mathrm{m}$ CMOS technology. The circuit is clocked at $8 \mathrm{MHz}$ and the measured SNDR is $56 \mathrm{~dB}$ in 
a $30 \mathrm{kHz}$ bandwidth.

A 6th-order two stage (4-2) bandpass $\Sigma \Delta$ inodulator is reported in [Hairapet46]. This modulator uses a pseudo-two-path SC resonator with one opamp. A dynamic range of $72 \mathrm{~dB}$ is achieved for this bandpass $\mathrm{A} / \mathrm{D}$ converter over a bandwidth of $2(\mathrm{X}) \mathrm{kHz}$ and an IF frequency of $3.25 \mathrm{MHz}$. This modulator is implemented in a $0.8 \mathrm{~mm}$ CMOS technol $\mathrm{gy}$ and consumes $7 \mathrm{~mW}$ from a $3 \mathrm{~V}$ supply.

In [Norman96], a $160 \mathrm{MHz}$ fourth-order bandpass $\Sigma \Delta$ modulator in a $1.8 \mathrm{\mu m}$ BiCMOS technology is reported. A dynamic range of $84 \mathrm{~dB}$ is achieved for a signal bandwidth of $2.5 \mathrm{MHz}$ centered at $5 \mathrm{MHz}$ in a medical ultrasound application. The architecture of this modulator is based on a SC integrator.

\section{Summary}

Oversampled sigma-delta converters are compatible with VLSI technolngy due to their architecture which contains simple analog circuitry followed by a complex digital signal processing unit. A narrow-band signal at IF can be digitized using a bandpass $\Sigma \Delta$ modulator. A simple transformation from lowpass to bandpass is achieved by a $z^{-1} \rightarrow-z^{-2}$ change of variable.

\subsection{Analog Circuit Design in a Standard Digital CMOS Process}

As CMOS VLSI technology advances to finer geometries, more analog functions of a mixed-signal chip find economic solutions in the digital domain. Advantages of digital circuits over analog include noise immunity, programmability, efficient scaling with technology, availability of excellent automated design tools, and a systematic test strategy. The boundary between digital and analog circuits is moving toward minimizing the amount of analog circuitry. However, interfacing with the physical world roquires analog circuits at least for implementing $A / D$ and $D / A$ converters.

A cost effective implementation of mainly digital mixed-signal circuits requires the analog circuits to be implemented in a standard digital CMOS process.

In CMOS. analog circuits are usually implemented using SC techniques due to their high circuit accuracy. SC circuits are traditionally implemented in CMOS processes where 
linear double-poly capacitors are available. Since a standard digital CMOS process does not offer a second layer of polysilicon, therefore, the double-poly option requires a process change to the mainstream digital CMOS technologies. In a predominantly digital mixed-signal chip, the extra cost associated with the special double-poly process option may not be justifiable.

To address this problem, a new sampled-data analog signal processing technique, called Switched-Current (SI) was proposed by Hughes [Hughes89]. This technique requires only MOSFET transistors in a dynamic current circuit configuration to perform analog signal processing. Thus, it is compatible with baseline digital CMOS technologies. However, reported performance results for SI circuits indicate poor accuracy due to settling error ard charge injection. In a recent publication [Nedver195], 11 bits of resolution is measured over the voice-band for a $5 \mathrm{~V} \Sigma \Delta$ modulator operating at $1.024 \mathrm{MHz}$. For the same modulator, SC circuits have achieved 14 bits.

In section 2.5.1, the SI techrique is described and in section 2.5.2, two high performance memory cells are discussed. Different techniques for implementing linear SC circuits in a single-poly digital CMOS process are presented in section 2.5.3.

\subsubsection{SI Circuit Technique}

Signal processing in the analog sampled-data domain requires four operations on the signals, namely: summation, inversion, scaling, and delay [Fiez90]. SC technique uses MOSFET transistors and floating capacitors as basic components to realize these operations.

A simple weighted current mirror, Figure 2.13, does the inversion, summation, and scaling operations. The output current $i_{o}$ is given by

$$
i_{o}(n)=-A\left[i_{1}(n)+i_{2}(n)\right] \text {. }
$$

where $A$ is the aspect ratio $(W / L)$ of $N_{2}$ with respect $r$, the aspect ratio of $N_{1}$.

A delay operation is achieved by adding a switch to a simple current mirror to isolate the gates of the MOSFETs $N_{1}$ and $N_{2}$ as shown in Figure 2.14.

This current sample-and-hold circuit is called a first generation memory cell [Hughes89] 


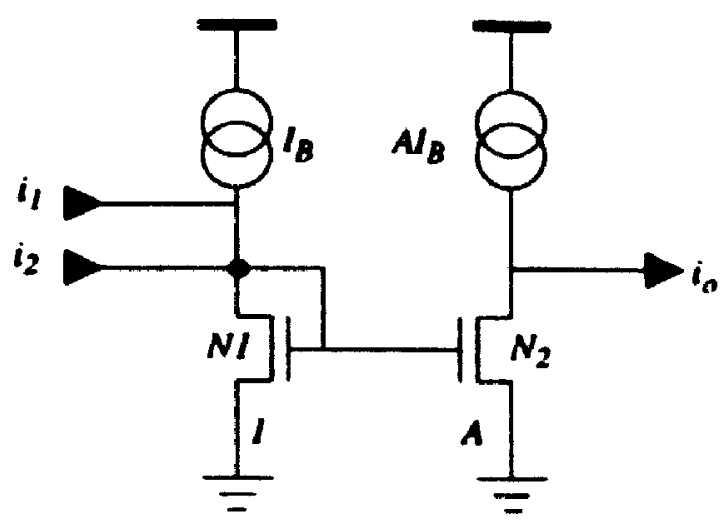

Figure 2.13: A weighted current mirror

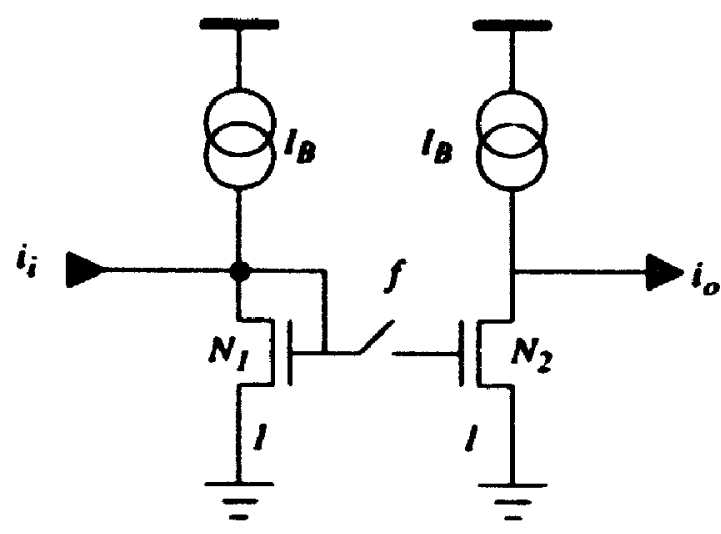

Figure 2.14: A first generation memory cell

and performs a half-delay operation. The transfer function $H(z)$ of this circuit is

$$
H(z)=\frac{i_{o}(z)}{i_{i}(z)}=-z^{-1 / 2} .
$$

A non-inverting damped integrator is realized using a full delay cell (two half-delay cells in cascade) in a feedback loop as shown in Figure 2.15 along with its corresponding 2phase non-overlapping clock.

The transfer function of this integrator is

$$
H(z)=\frac{A z^{-1}}{1-B z^{-1}} .
$$

which corresponds to the Forward Euier transformation $\left(s \rightarrow 1 / T\left[\left(1-z^{-1}\right) / z^{-1}\right]\right)$ of 


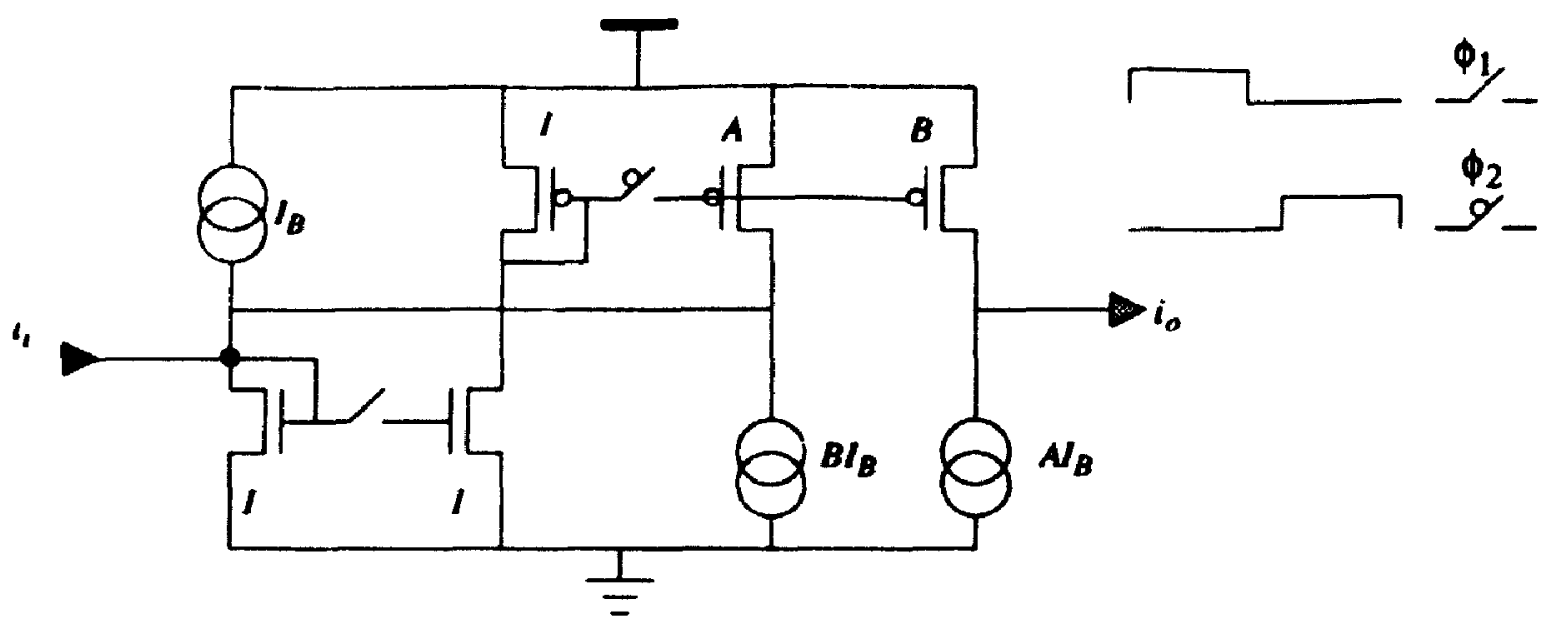

Figure 2.15: First generation SI integrator

the following continuous time integrator.

$$
H(s)=\frac{a_{0}}{1+\frac{s}{\omega_{0}}}
$$

$a_{0}$ is the DC gain and $\omega_{0}$ is the cut-off frequency.

The sampled data integrator of (2-47) corresponds to the continuous time integrator of (248) with a phase shift $\left(e^{(-j \omega T) / 2}\right)$ [Battersby93] and

$$
a_{0}=\frac{A}{1-B} \quad \omega_{0}=\frac{2}{T}\left(\frac{1-B}{1+B}\right)
$$

The sensitivity of $x$ with respect to variation in $y$ is defined as

$$
\left.S\right|_{y} ^{x}=\left(\frac{y}{x}\right) \frac{\partial x}{\partial y}
$$

The sensitivity of the cut-off frequency and DC gain with respect to variation in $B$ is found to be:

$$
\left.S\right|_{B} ^{a_{0}}=\left.\frac{B}{1-B} \quad S\right|_{B} ^{\omega_{0}}=\frac{2 B}{1-B^{2}}
$$

Thus, the performance of this integrator with a low damping factor $(B \rightarrow 1)$ is very sensitive to variation in the coefficient $B$ which is determined by transistor matching. 
The second generation memory cell [Hughes90], shown in Figure 2.16, solves the MOSFET mismatch problem by utilizing the same transistor for sampling the signal as well as holding it.

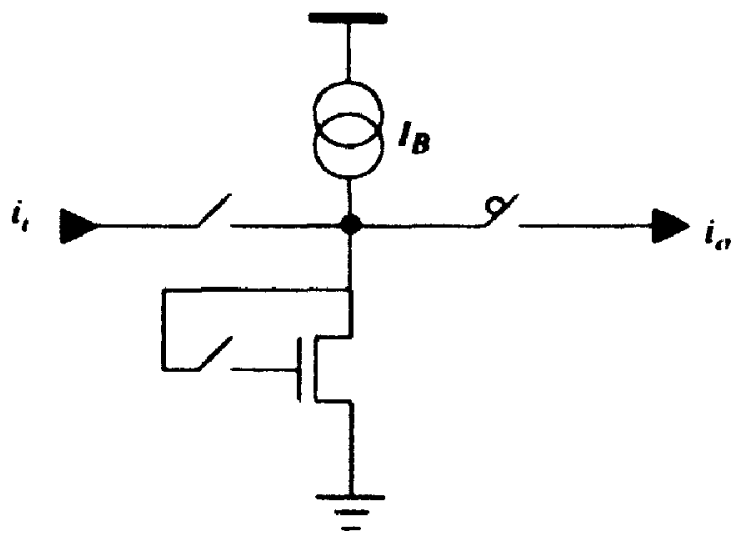

Figure 2.16: Second generation SI memory cell

A lossless non-inverting integrator circuit constructed using a second generation SI

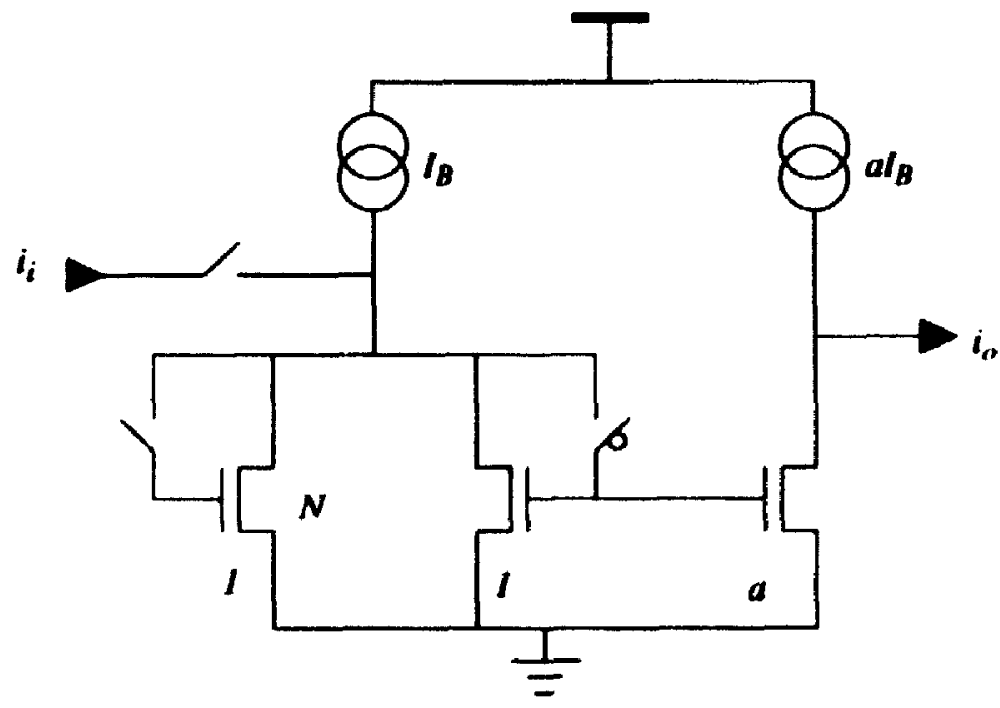

Figure 2.17: A second generation non-inverting lossless integrator

memory cell is shown in Figure 2.17. The transfer function of this circuit is

$$
H(z)=\frac{a z^{-1}}{1-z^{-1}}
$$

A damped non-inverting integrator is obtained by adding feedback to the lossless 
integrator as shown in Figure 2.18.

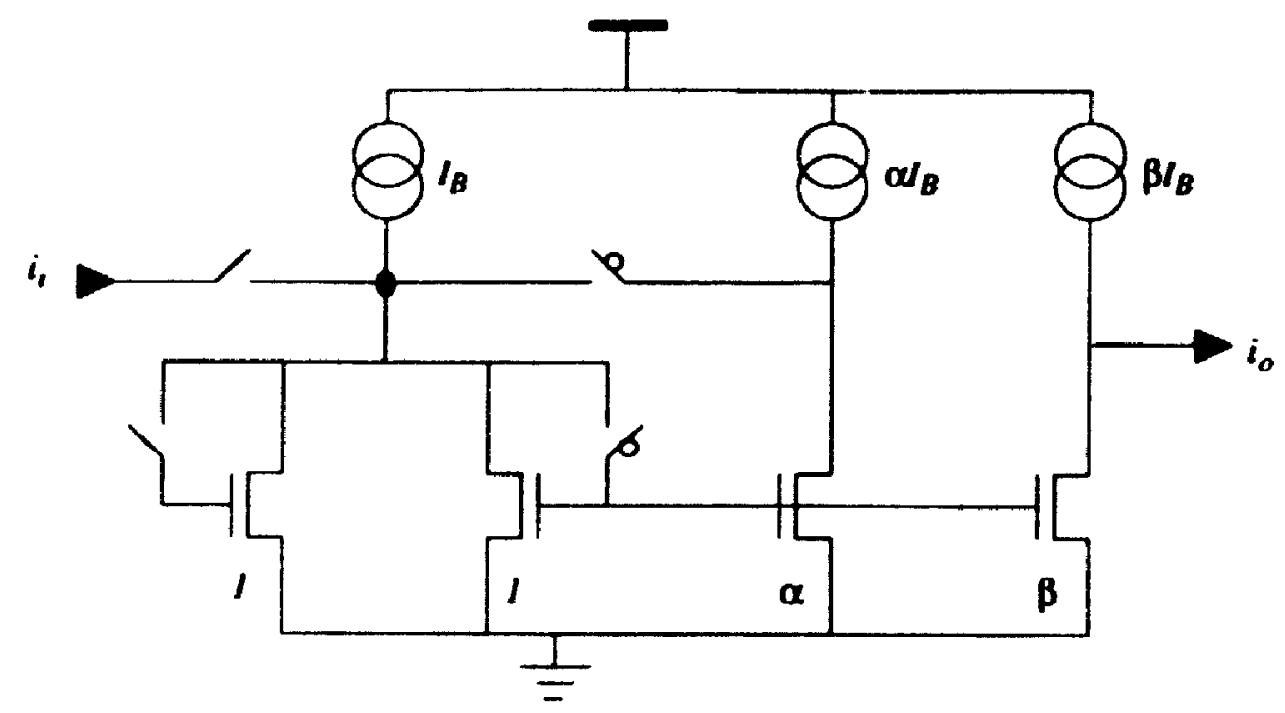

Figure 2.18: Second generation damped integrator

The transfer function of the above damped integrator is

$$
H(z)=\frac{A z^{-1}}{1-B z^{-1}}
$$

where

$$
A=\frac{\beta}{1+\alpha} \quad B=\frac{1}{1+\alpha} .
$$

Cut-off and DC gain sensitivity with respect to $\alpha$ and $\beta$ are

$$
\begin{array}{cc}
\left.S\right|_{B} ^{a_{0}}=1 & \left.S\right|_{B} ^{\omega_{0}}=0 \\
\left.S\right|_{B} ^{a_{0}}=-1 & \left.S\right|_{B} ^{\omega_{0}}=\frac{2}{2+\alpha} .
\end{array}
$$

Both $\omega_{0}$ and $\alpha_{0}$ exhibit low sensitivity to variations in $\alpha$ and $\beta$.

\subsubsection{High Performance Memory Cells}

An ideal SI memory cell must exhibit infinite input conductance $g_{i}$ during the sampling phase and infinite output resistance $r_{o}$ during the hold phase. However, the simple memory cell in Figure 2.16 has a finite input impedance $g_{m}$ and output resistance $1 / g_{d s}$. 
The accuracy of a SI circuit is proportional to $g_{i} r_{j}=g_{m} / g_{d s}$. There are many circuit techniques proposed to improve the accuracy of SI circuits. Here. two prominent methods. regulated cascode [Toumazou90] and $S^{2} 1$ [Hughes93] are discussed.

\section{Regulated Cascode Memory Cell}

The regulated cascode memory cell, illustrated in Figure 2.19, is based on a regulated cascode current mirror [Sackinger87] that improves the output conductance of the memory cell by a factor which is higher than that of simple cascoding.

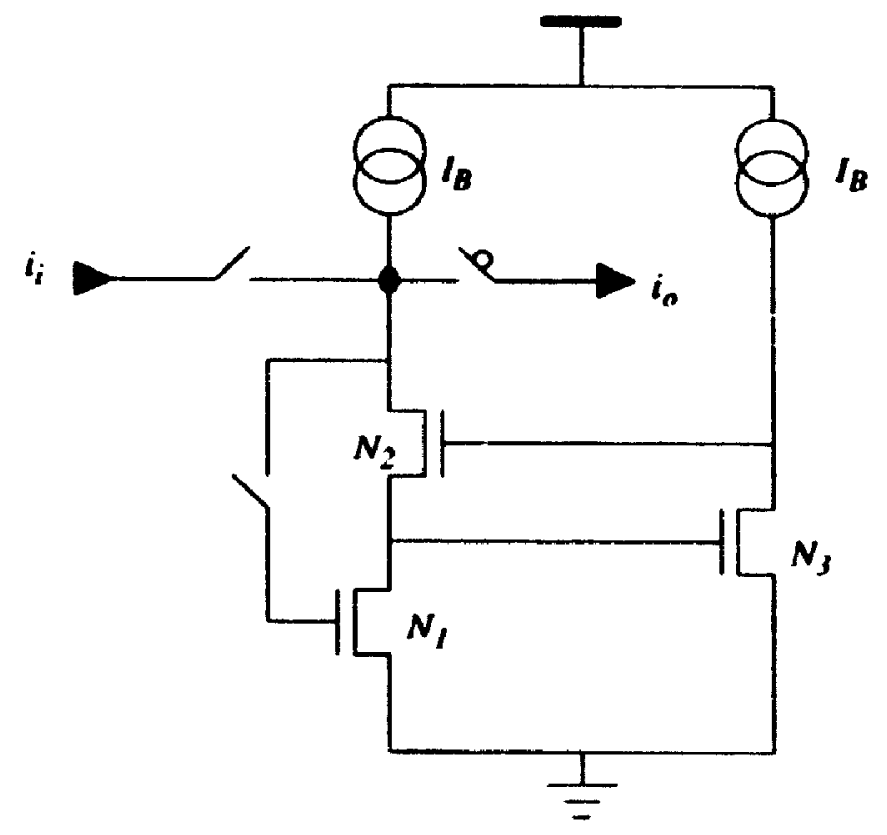

Figure 2.19: Regulated cascode memory cell

This cell operates as follows. During the sampling phase the voltage on the gate of $N 1$ reaches $v_{G S 1}$ to maintain a drain current of $t_{B}+i_{i}$. In the hold phase, the gate of $N I$ is isolated and the sampled current is fed to the output. The negative feedback loop formed by transistors $N 2$ and $N 3$ keeps the voltage $v_{D S 1}$ constant at a value of $v_{G ; S 3}$.

The input and output conductance of this circuit are

$$
g_{i}=g_{m 1} \quad r_{o}=\frac{g_{m 2} g_{m 3}}{g_{d s 1} g_{d s 2} g_{d s 3}}
$$

Compared to a simple current copier, the value of $g_{i} r_{o}$ for a regulated cascode is higher 
by a factor of $\left(g_{m 2} g_{m 3}\right) /\left(g_{d s 2} g_{d s 3}\right)$.

\section{$S^{2} I$ Memory Cell}

A two-step memory cell, called $S^{2} I$ cell, and its asscciated clock waveforms are shown in Figure 2.20.
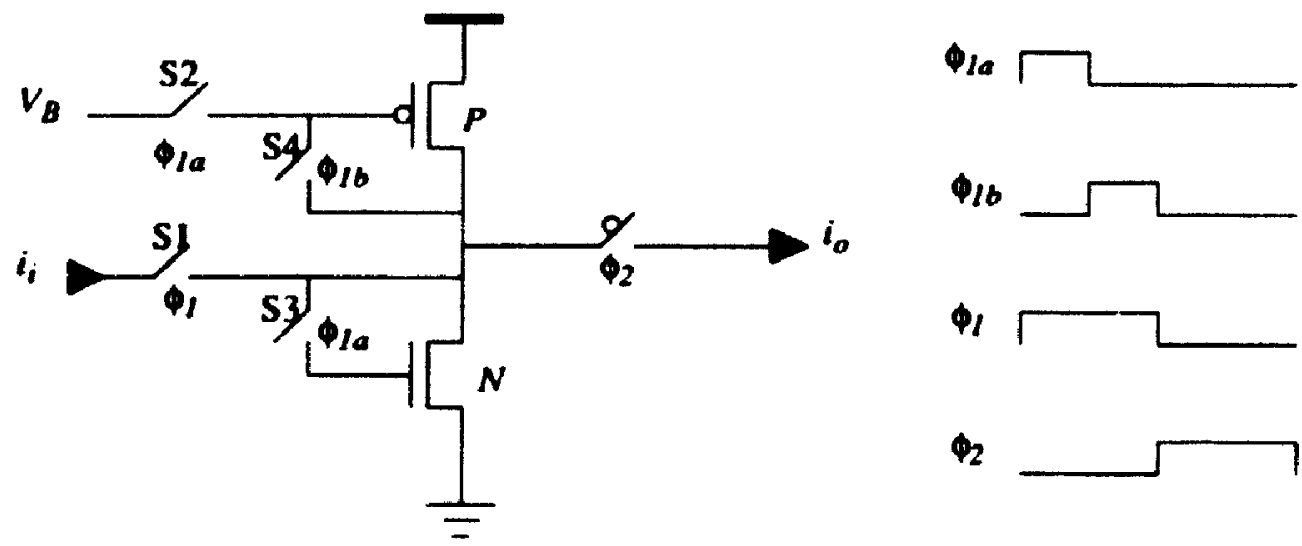

Figure 2.20: $\mathrm{S}^{2}$ I memory cell

On phase $\phi_{1 a}$, the coarse memory transistor $N$ is diode connected and the input current is sampled. The bias current is provided by transistor $P$ during this phase. During $\phi_{1 b}$, the gate of $N$ is opened and its drain-source current will be sustained (parasitic $C_{g s}$ holds the gate to source voltage). Circuit non-idealities and charge injection from sampling switch $S 1$ cause the stored current in $N$ to be different than the input current. The fine memory transistor $\boldsymbol{P}$ is now diode connected and will sample the difference between the actual input current $i_{i}$ and the stored current $i_{d s 1}$. This difference is the error current caused during the coarse sampling phase. On phase $\phi_{2}$, the stored current (in $N$ ) is subtracted from the error current (in $P$ ) and provides an error free output current $i_{o}$.

\subsubsection{Linear SC Circuit Techniques in a Digital CMOS Process}

As previously discussed, implementation of the linear SC circuits requires linear floating capacitors. In standard digital CMOS processes, where a linear double-poly capacitor is not available, parasitic poly-to-metal or metal-to-metal capacitors may be used as linear capacitors. The parasitic capacitance per unit area is typically low $\left(0.05 \mathrm{fF} / \mathrm{\mu m}^{2}\right)$ compared to that of a poly-poly capacitor, which is $1 \mathrm{fF} / \mathrm{um}^{2}$. The penalty of using parasitic capacitances is the increase in silicon real estate (by a factor of almost 20) that 
may not be acceptable if the total capacitance used in the circuit is large.

Another type of capacitor available in CMOS processes is the MOSFET gate capacitor, which is the one exploited in SI circuits. The capacitance per unit area of a MOSFET gate capacitor is typically higher (by a factor of $1.5-2.5$ ) than the capacitance per unit area of a poly-poly capacitor. However. MOSFET gate capacitance exhibits a non-linear behavior as shown in Figure 2.21 .

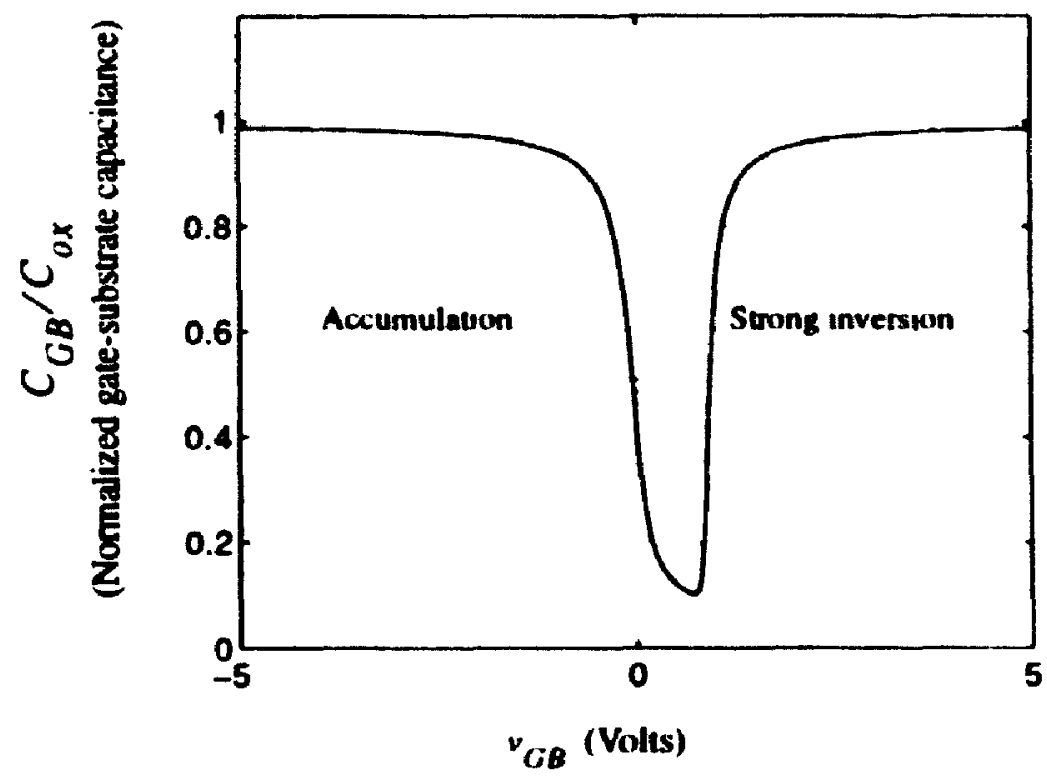

Figure 2.21: nMOSFET CV plot

Reported research in the area of employing MOSFET gate capacitance to realize a linear SC circuit is reviewed in the following section.

\section{Weakly Non-linear MOSFETs}

As can be seen from the CV characteristics of an nMOSFET shown in Figure 2.21, a relatively linear capacitor is achieved by operating the MOSFET in strong inversion $\left(v_{G S}>V_{t}\right)$ or accumulation $\left(v_{G S}<0\right)$ regimes. Techniques to bias the MOSFETs in strong inversion or accumulation to reduce distortion in the SC filter design were first reported in [Montoro88].

A theoretical analysis of the harmonic distortion caused by the weakly non-linear MOSFET capacitors biased in strong inversion or accumulation is described in (Behr'92). It is shown that distortions due to these capacitors are technology independent. The 
voltage coefficient $\alpha$ (called $v_{c c}$ in [Behr92]) for gate MOSFET capacitor is derived from

$$
\alpha=\frac{1}{C_{g}}\left|\frac{d C_{g}}{d v_{G B}}\right| .
$$

where $C_{g}$ is the gate capacitance and $v_{G B}$ is the gate to bulk voltage. For a MOSFET gate capacitor biased at $v_{G B}=V_{R}$. the voltage coefficients in strong inversion and in the accumulation region are given respectively by

$$
\begin{aligned}
\left.\alpha\right|_{s i r} & =\frac{2 \phi_{t}}{\left(V_{R}-V_{t}\right)^{2}} \\
\left.\alpha\right|_{a c c} & =\frac{2 \phi_{t}}{\left(V_{R}-V_{F B}\right)^{2}} .
\end{aligned}
$$

Here, $\phi_{t}$ is the thermal voltage and $V_{F B}$ is the flat band voltage. A voltage capacitance coefficient of $17 \mathrm{kppm} / \mathrm{V}$ is obtained for a MOSFET with a $V_{,}$of $0.8 \mathrm{~V}$, biased $2.5 \mathrm{~V}$ in strong inversion.

A simple damped integrator circuit is used to test MOSFET capacitors. Measured THD results are in good agreement with computed results showing that approximately $-40 \mathrm{~dB}$ THD is achievable using a $3 \mathrm{~V}$ signal swing.

In another study [Schneider94], an explicit formula is derived for the harmonic distortion in SC circuits with a weakly non-linear capacitor. It is shown that hamonic distortions as low as $-40 \mathrm{~dB}$ to $-60 \mathrm{~dB}$ can be obtained in SC filters using MOSFETs biased in strong inversion or accumulation as linear capacitors. A single-ended discrete SC biquad filter is implemented. using a Motorola P5N40 transistor as capacitor, to verify the theoretical analysis.

\section{SC Circuits as Linear Charge Processors}

In [Bermudez92], the basic operation of a SC circuit is considered as a charge mirror cell cr mposed of capacitors and an opamp. The charge processing is linear and is independent of the non-linearity of the capacitors involved. 
A charge mirror is shown in Figure 2.22. A charge $q_{A}$ injected at the inverting terminal of the opamp will appear on capacitor $C_{A}$ with the following relationship between charge and voltage:

$$
q_{A}=C_{A 0} f_{A}(v) v
$$

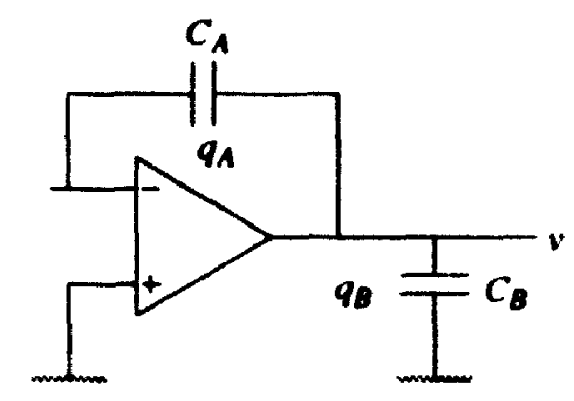

Figure 2.22: A charge mirror cell

A non-linear capacitor $C_{X}$ is described by [Lee85]

$$
C_{X}=C_{X 0} f_{X}(v)
$$

The parameter $C_{X 0}$ is the capacitance at zero voltage and is proportional to the area of the capacitor.

The charge on capacitor $C_{B}$, in Figure 2.22, is

$$
q_{B}=C_{B d} f_{B}(v)
$$

If capacitors $C_{A}$ and $C_{B}$ have the same non-linearity function, then

$$
\frac{q_{B}}{q_{A}}=\frac{C_{B 0}}{C_{A 0}}=\frac{\operatorname{area}\left(C_{B}\right)}{\operatorname{area}\left(C_{A}\right)}
$$

This is a linear charge mirror and the gain is defined by the area ratio.

A typical SC building block which performs summation, scaling and delay operation on two charge signals is shown in Figure 2.23.

In the charge domain, the operation is linear from input to output. If the external signals are in the voltage domain, a linear input $V / Q$ and output $Q / V$ converters are required. 


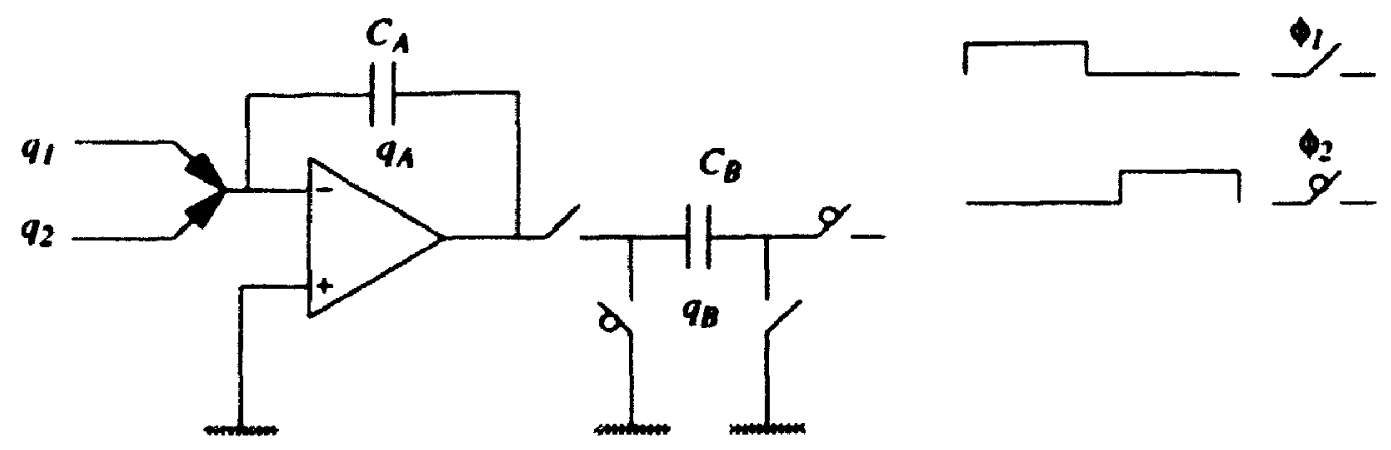

Figure 2.23: A SC building block

This means any capacitor connected to either inputs or output (voltage) must be a linear capacitor.

\section{A High-Linearity MOSFET SC Branch}

A high-linearity SC circuit branch using MOSFET capacitors, proposed in [Yoshizawa95], is shown in Figure 2.24. In this circuit, MOSFETs are biased in a weakly non-linear region (accumulation) and the non-linearity effects are canceled to a first-order. because the two capacitors are connected in series and back-to-back.

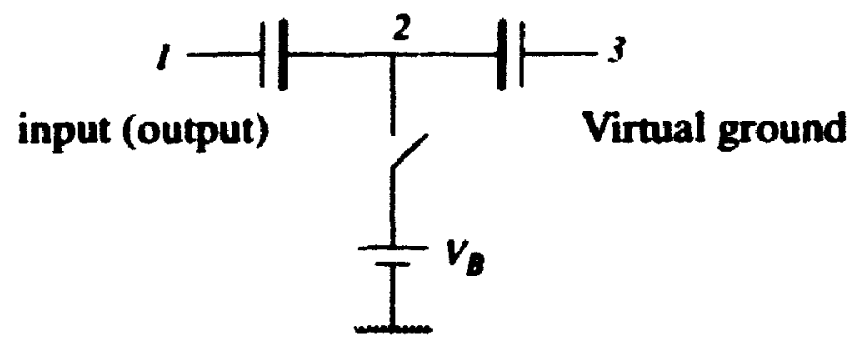

Figure 2.24: The MOSFET SC branch

This MOSFET capacitor branch can be used in a SC circuit as shown in Figure 2.25. An expression similar to (2-61) is used for the weakly non-linear capacitors $C_{A}$ and $C_{B}$ and it is assumed that $C_{A 0}=C_{B 0}=C$. If $V_{i n} / 2 * V_{B}$, it can be shown that the charge delivered by the SC branch during $\phi_{2}$ is

$$
\Delta q=\frac{C}{2}\left[f\left(V_{B}\right)+\left.\frac{d f}{d v}\right|_{V_{u}}\right] V_{i n}(n)
$$


Since $V_{B}$ is a constant voltage. the term in the bracket is a constant. Therefore, the effective capacitance term is a constant and $\Delta q$ charge is a linear function of the $V_{\text {in }}(n)$ voltage.

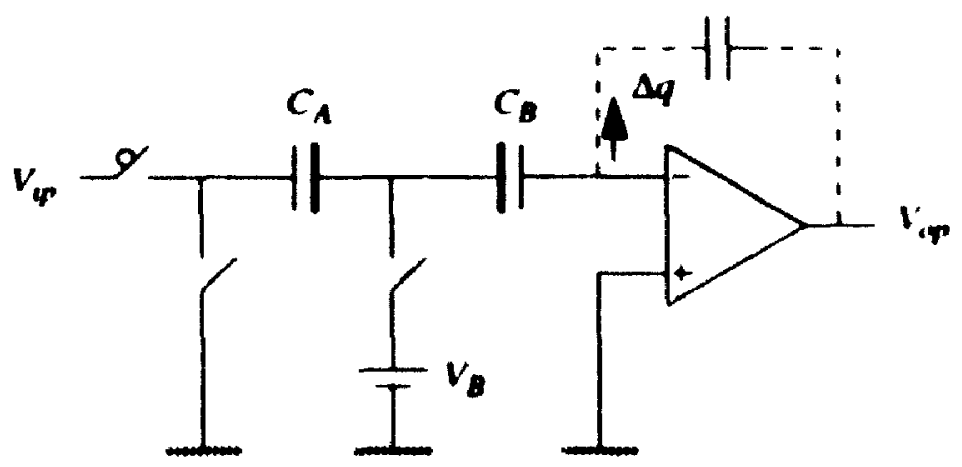

Figure 2.25: A SC circuit using the MOSFET SC branch

An experimertal SC amplifier with a gain of 10 was implemented using pMOS capaciturs in accumulation to verify the linearity of the above SC branch (Yoshizaway6). For $V_{B}=1.5 \mathrm{~V}$ and a $400 \mathrm{mVpp}$ input signal at $1 \mathrm{kHz}$, the largest harmonic was $72 \mathrm{~dB}$ below the fundamental of the input signal.

\section{Semanery}

Sampled-analog circuits can be realized in standard digital CMOS process using SI or SC circuit techniques. In SC design, linear capacitors can be achieved using parasitic poly-tometal or metal-to-metal capacitors or using MOSFET gate capacitors biased in strong inversion or accumulation regimes.

\subsection{A Comparative Study of SC and SI for Low-Voltage Applications}

Supply voltage down-scaling was shown to be essential in future deep submicron VLSI circuits to prevent problems caused by breakdown voltage, hot electron effects, and power dissipation. A reduced supply voltage decreases the voltage signal swing which in turn makes the design of high-speed wide dynamic range SC circuits a challenge. In SI circuits, the signal is in the current domain and this originally led some reseurchers to believe that the SI technique is more suitable for low-voltage analog design [Hughes(y)al [Fiez(X)] [Battersby91]. Further research [Crawley92] [Temes93] [Bazarjani93] indicated that SI 
would not benetit from supply voltage scaling. In this section. limits to low-voltage SC and SI are explored and it will be shown that SI exhibits no clear advantage over SC for low-voltage analog function. In fact, analysis of dynamic range (for a given supply voltage) indicates that SC circuits achieve a higher dynamic range than SI circuits.

A SC circuit consists of capacitors. opamps, and suitches and a SI circuit comprises current mirrors and analog switches. In section 2.3, limits to low-voltage operation of the opamp. switch, and current mirror were investigated. A transmission gate switch requires a supply voltage of greater than $2 V_{1}+2 V_{o n}$. Both opamp and current mirror need a supply voltage of greater than $V_{1}+2 V_{\text {on }}$. Therefore, the minimum supply voltages required for proper operation of either SC circuits or SI circuits are the same.

An important performance parameter for low-voltage operation is the maximum achievable circuit dynamic range as defined by (2-25). For dynamic range comparison, the SC integrator circuit shown in Figure 2.7 and the SI integrator circuit shown in Figure 2.17 are considered.

The maximum output voltage swing in the SC integrator is determined by the linear voltage swing at the output of the opamp. For a two-stage opamp. as shown in Figure 2.26. the maximum output voltage swing is

$$
V_{\text {swing (opamp) }}=V_{D D}-2 V_{o n} \text {. }
$$

where $V_{o n}$ is the minimum $v_{D S}$ voltage needed to keep the output transistors $N 4$ and $P 3$ in the opamp (Figure 2.26) in saturation. The noise in a SC circuit is limited by $k T / C$ and the circuit dynamic range is

$$
\left.\therefore \quad D R\right|_{S C}=\frac{V_{D D}-2 V_{o n}}{\sqrt{(8 k T) / C_{S}}} .
$$

In the SI circuit, during the sampling phase the minimum voltage on the gate of $N$ is $V_{t}+V_{o n}$ and the maximum voltage on the gate of $N$ can rise to $V_{D D}-V_{o n}$, to keep $P$ in saturation. The voltage signal swing on the gate of $N$ is

$$
V_{\text {swing (SI) }}=V_{D D}-V_{1}-2 V_{\text {on }} \text {. }
$$




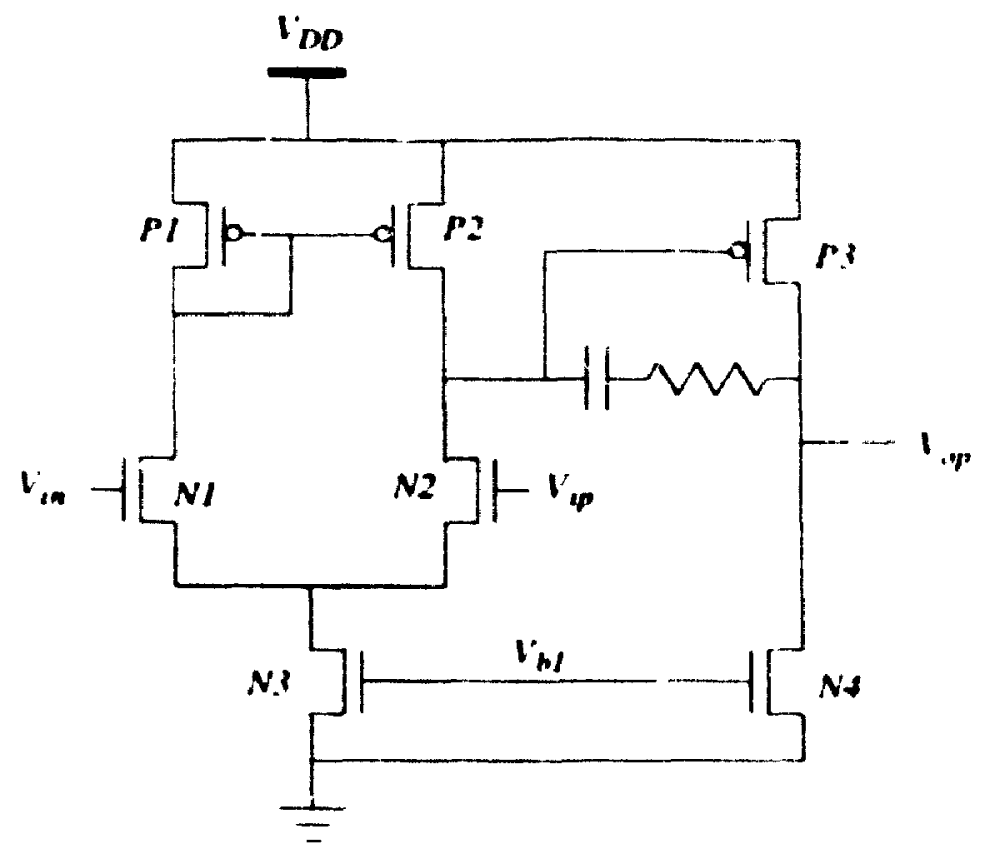

Figure 2.26: A two-stage pole-splitting opamp

The minimum stored voltage on the gate capacitance of the nMOSFET transistor $N I$ must be greater than the thermal noise $k T / C_{g}$, where $C_{g}$ is the gate capacitance of $N 1$. The dynamic range of the $S I$ integrator is

$$
\left.D R\right|_{S I}=\frac{v_{D D}-V_{1}-2 V_{o n}}{\sqrt{(8 k T) / C_{X}}}
$$

For example, assuming $C_{s}=C_{g}, v_{t}=0.6 \mathrm{~V}, v_{D D}=1.6 \mathrm{~V}$. and $v_{u n}=11.2 \mathrm{~V}$ the DR of the SC integrator is $6 \mathrm{~dB}$ larger than the DR of the SI integrator.

\subsection{Summary}

In this chapter, CMOS scaling in deep submicron tahnologies was reviewed and It was mentioned that supply voltage has started to scale down due to reliability issues. Along with the supply voltage. threshold voltage must be xualed down to achieve high speed circuits. Threshold voltage scaling issues due to non-scalability of subthreshold swil.g were discussed. Limits to digital and analog supply voltage sealing were dixussed and 11 was shown that a large margin for supply voltage reduction exists for digital (MOS. Dynamic power consumption in CMOS logic is reduced quadratically with supply voltage 
reduction factor. A first-order analysis showed that for a given dynamic range and . peed, power consumption of an analog circuit is independent of the supply voltage. Research in the area of low-voltage digital and analog circuits was presented.

Then, $\Sigma \Delta$ modulators, which are the benchmark circuits in this thesis, were introduced and reported high-speed bandpass SC $\Sigma \Delta$ modulators were reviewed.

Finally, analog circuit techniques that allow mixed-signal circuit to be implemented in a standard digital CMOS process were presented. 


\section{Chapter 3}

\section{Low-Voltage SC Design with Low- $V_{t}$ MOSFETs}

In Chapter 2, CMOS scaling was reviewed and it was discussed that in deep submicron technologies the supply voltage is scaling down to assure device reliability. In conjunction with supply voltage, MOSFET threshold voltage must be reduced to attain high-speed circuits. However. lowering the threshold voltage of MOSFET transistors will increase the subthreshold off-current which raises the standby power dissipation in digital circuitry and limits the accuracy of analog SC circuits. It was discussed that scaling the supply voltage along with the threshold voltage reduces the total power dissipation in digital CMOS. Low-voltage anc:og SC circuit techniques using low- $V$, MOSFETs are investigated in this chapter.

Low- $V$, MOSFETs are available in advanced $0.1 \mu \mathrm{m}$ CMOS technologies. This chapter tarts by presenting two different methods of achieving low- $V$, MOSFETs in the current CMOS processes; the first scheme may involves a process change, and the second method uses circuit techniques. Accuracy degradation in SC circuits due to the subthreshold leakage current of low- $V$, MOSFET transmission gate switch is analyzed next. Then, methods for reducing the subthreshold leakage in analog switches are discussed and two new switch topologies, the series ransmission gate and composite switch [Bazarjani94b]. addressing this problem are proposed. Finally, the design and measured results for two low-voltage SC $\Sigma \Delta$ modulators are presented. The first design is a $2.25 \mathrm{~V}$ second-order $\Sigma \Delta$ modulator designed in a $0.8 \mu \mathrm{m}$ BiCMOS technology using short-channel $(0.6 \mu \mathrm{m})$ MOSFET switches. This modulator operates at a $2.5 \mathrm{MHz}$ clock rate and achieves $92 \mathrm{~dB}$ 
SNDR over the C-message weighted telephony bandwidth ( $60 \mathrm{~Hz}-5 \mathrm{kHz}$ ). The second design is a $1 \mathrm{~V}$ first-order $\Sigma \Delta$ modulator implemented in a $0.5 \mu \mathrm{m}$ CMOS process using low- $V$, natural MOSFETs. This modulator is clocked at $1 \mathrm{MHz}$ and has $54 \mathrm{~dB}$ dynamic range for an oversamplir of 128 (voice band $4 \mathrm{kHz}$ ) and consumes about $100 \mu \mathrm{W}$.

\subsection{A Low-V, Process}

As channel lengths of MOSFET transistors are shrunk to $0.5 \mu \mathrm{m}$ and below, buriedchannel pMOSFET devices with strong short-channel effects (SCEs) must be replaced with surface channel devices. SCE is a combination of several 2-D phenomena in short channel MOSFETs and is expressed as $V_{t}$ roll-off due to channel shortening [Tsividis87]. Fabrication of surface-channel MOSFETs requires a dual poly gate process technology where $\mathrm{n}^{\boldsymbol{+}}$-poly is used for rMOSFETs and $\mathrm{p}^{\boldsymbol{+}}$-poly is used for pMOSFETs. In such technologies, the natural threshold voltages of the transistors are set by the well implants and are given by [Chen90],

$$
V_{1}=V_{F B}+2 \phi_{F}+\sqrt{2 q \varepsilon_{s i} N_{W}\left(2 \phi_{F}\right)} / C_{o x}
$$

where $V_{F B}$ is the flat-band voltage, $\phi_{F}$ is the bulk Fermi potential, $N_{W}$ is the device well doping concentration, and $C_{o x}$ is the gate specific capacitance. $V_{F B}$ and $\phi_{F}$ are related to process parameters and physical constants by

$$
\begin{gathered}
v_{F B}=\phi_{M S}-\frac{\hat{Q}}{C_{o x}} \\
\phi_{F}=\frac{k T}{q} \ln \left(\frac{N_{W}}{n_{i}}\right),
\end{gathered}
$$

where $\hat{Q}$ is the fixed charge density and $\phi_{M S}$ is the gate-to-substrate workfunction. In an $\mathrm{n}^{+} / \mathrm{p}^{+}$dual poly gate CMOS technology, the threshold voltages of the natural MOSFETs are symmetric. This is due to the symmetrical gate-to-substrate workfunction of the MOSFETs, as shown in Figure 3.1.

The $\phi_{M S}$ for nMOSFETs and pMOSFETs are 


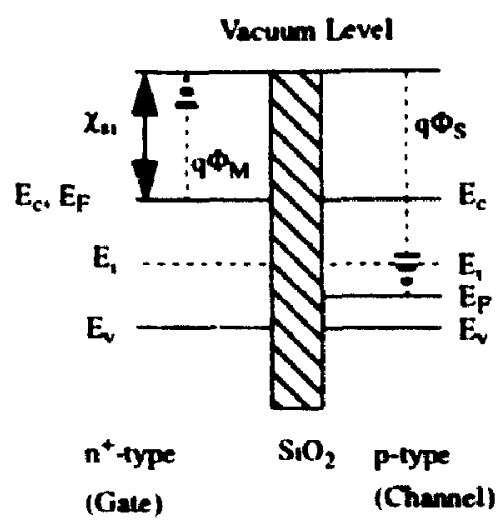

aMOSFET

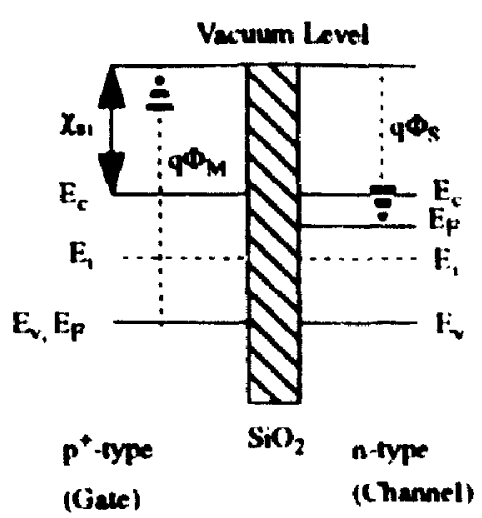

PMOSFET

Figure 3.1: Gate-substrate workfunction of nMOSFET and pMOSFET in an $\mathrm{n}+/ \mathrm{p}+$ dual poly gate CMOS process

$$
\begin{aligned}
& \phi_{\left.M S\right|_{n M O S F E T}}=-\left(\frac{E_{g}}{2 q}+\phi_{F}\right) \\
& \phi_{\left.M S\right|_{p M O S F E T}}=\left(\frac{E_{g}}{2 q}+\phi_{F}\right) .
\end{aligned}
$$

Here, $E_{g}$ is the bandgap energy which is about $1.124 \mathrm{eV}$ at room temperature and $q$ is the charge of an electron that is $1.602 \times 10^{-19} \mathrm{C}$.

Example 3.1: Assuming a fixed charge density of $2 \times 10^{10} \mathrm{q} / \mathrm{cm}^{3}$ and device well dopings of $4 \times 10^{16} \mathrm{~g} / \mathrm{cm}^{3}$. the natural threshold voltage of MOSFETs in a typical 0.5 $\mu \mathrm{m}$ CMOS technology with gate oxide thickness of $12 \mathrm{~nm}$ is found to be $V_{1}=220 \mathrm{mV}$.

In some dual $\mathrm{n}^{+} / \mathrm{p}^{+}$poly gate processes [Sun92], it is possible to mask out the thr shold adjust implant and obtain the low- $V$, natural MOSFETs. In the next section, device characteristics of an experimental $1 \vee C M O S$ process [Bazarjani95b] are described.

\section{Experimental Natural MOSFETs}

Natural threshold voltage CMOS transistors have been fabricated using a $0.5 \mu \mathrm{m}$ CMOS process. This process does not require any threshol 1 adjust implant (for natural MOSFETs). For $1 \mathrm{~V}$ operation, this process can be further simplitied by eliminating the steps required for hot-carrier reduction i.e., Lightly-Doped-Drain (LDD). 
The threshold voltages of $0.5 \mu \mathrm{m}$ channel length nMOSFET and pMOSFET devices are measured to be $202 \mathrm{mV}$ and $197 \mathrm{mV}$ respectively. Figure 3.2 and Figure 3.3 show the $I_{D}$ and $G_{m}$ versus gate voltage for a $20 \mu \mathrm{m} / 0.5 \mu \mathrm{m}$ nMOSFET and a $20 \mu \mathrm{m} / 0.5 \mu \mathrm{m}$. pMOSFET biased at $V_{D S}=0.1 \mathrm{~V}$ respectively.

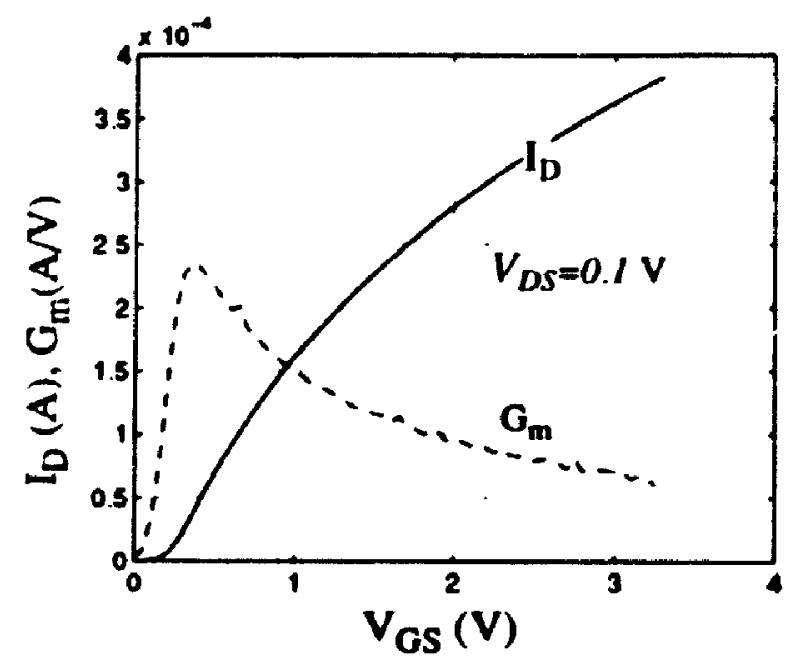

Figure 3.2: Measured $I_{D}(A)$ and $G_{m}(A / V)$ versus $V_{G S}(V)$ for "natural" nMOSFET

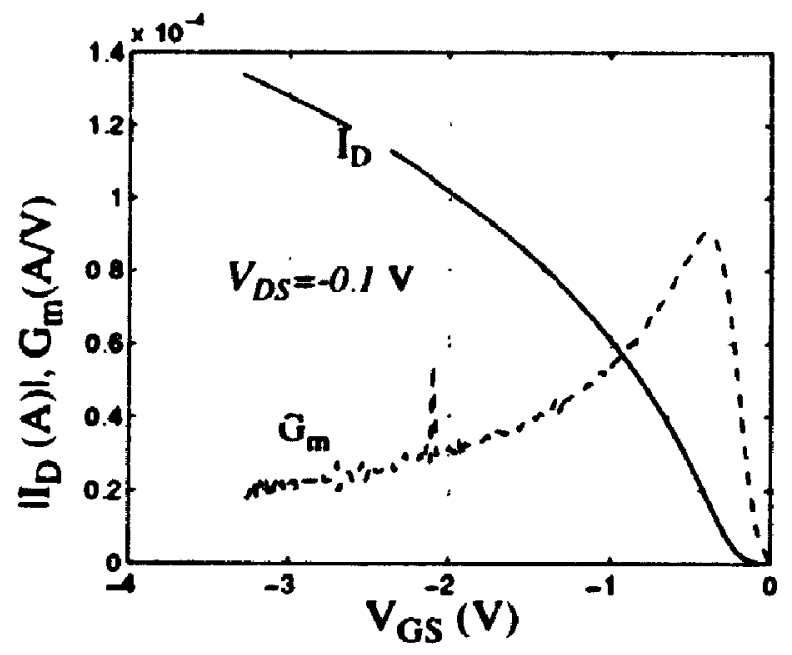

Figure 3.3: Measured $I_{D}(A) I$ and $G_{m}(A / V)$ versus $V_{G S}(V)$ for "natural" pMOSFET

Subthreshold slopes are measured to be $78 \mathrm{mV} /$ decade as illustrated in Figure 3.4.

\section{Summary}

Low- $V$, natural threshold voltage MOSFETs can be fabricated as a by-product of a dual 


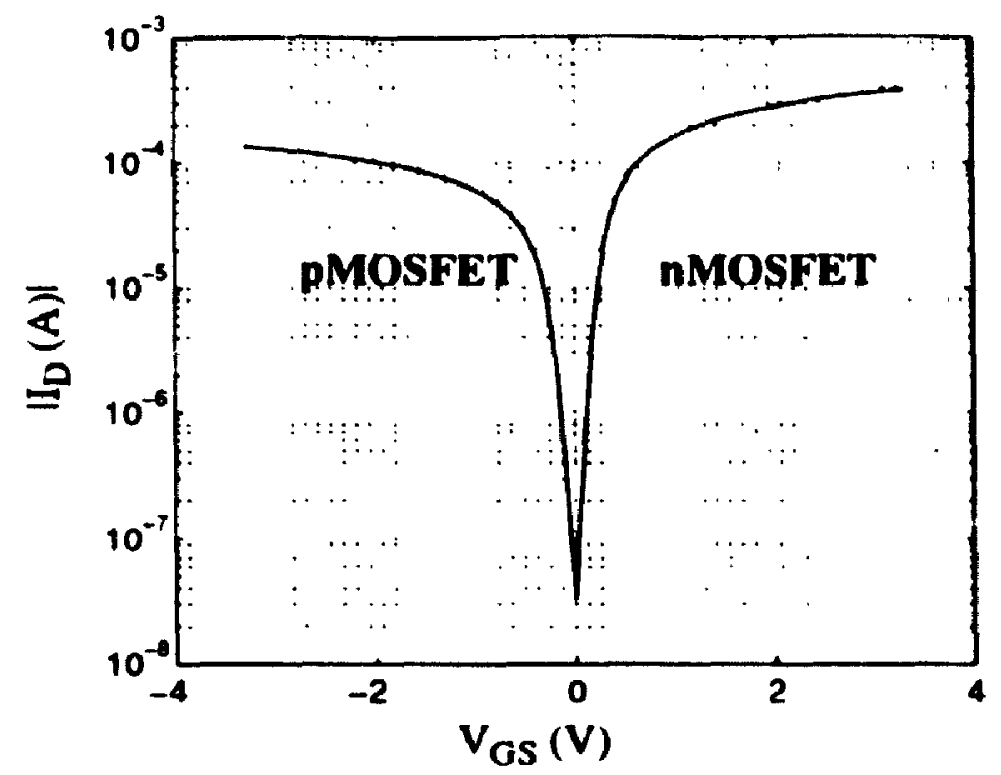

Figure 3.4: Measured subthreshold characteristics of natural nMOSFET and pMOSFET

poly gate CMOS process where the threshold voltage is set by a well implant. The natural MOSFET is obtained by selectively shielding the threshold adjust implant or removing the steps for threshold adjust for the whole wafer. Measured results, for a $0.5 \mu \mathrm{m}$ technology. indicate that typical values for $V_{1}$ and $S$ are about $200 \mathrm{mV}$ and $80 \mathrm{mV} /$ decade respectively. These low- $V$, natural MOSFETs are suitable for low-voltage $\left(V_{D, n}-1 \mathrm{~V}\right)$ mixed-signal design. In a dual- $V$, process, the low- $V$, transistors can be used to achieve higher speed in digital circuits. In Chapter 5, application of a low- $V$, MOSFET to the design of a high-swing linear MOSFET capacitor will be discussed.

\subsection{Circuit Technique for $V_{t}$ Reduction}

If a low- $V$, process is not available, circuit techniques must be used to reduce the threshold voltage. In this section, methods of reducing the threshold voltage by $1(x) \mathrm{mV}$ to $200 \mathrm{mV}$ are discussed.

\section{(I) Low-Vt Short-Channel Devices: Taking advantage of SCEs}

As the channel length of a MOSFET transistor is made smaller than a critical value $\left(L_{\min }\right)$, several 2-D phenomena known as short channel effects (SCEs) will occur. The onset of SCEs is empirically given [Brews80] by 


$$
L_{\min }=8.8\left[r_{j} t_{o \lambda}\left(W_{S}+W_{D}\right)^{2}\right]^{1 / 3}
$$

Normally, process engineers select the minimum feature size of the technology $\left(L_{0}\right)$ to be slightly greater than $L_{\text {min }}$. One of the characteristics of short channel devices is that threshold voltage rolls off quickly as channel length is reduced below $L_{\min }$. Figure 3.5 illustrates the threshold voltage as a function of channel length, for a generic $1.5 \mu \mathrm{m}$ C.MOS technology, based on the charge-sharing model approximation formula [Tsividis87] given by

$$
V_{l}(\dot{L})=V_{n}\left(1-\frac{\alpha_{1}}{L} \sqrt{\frac{2 \varepsilon_{s i} \phi_{B}}{q N_{A}}}\right)
$$

Here, $V_{(0)}$ is the long channel threshold voltage, $L$ is the channel length, $\phi_{B}$ is the surface potential at the onset of strong inversion which is approximately $2 \phi_{F}$, and $\alpha_{1}$ is a fitting parameter.

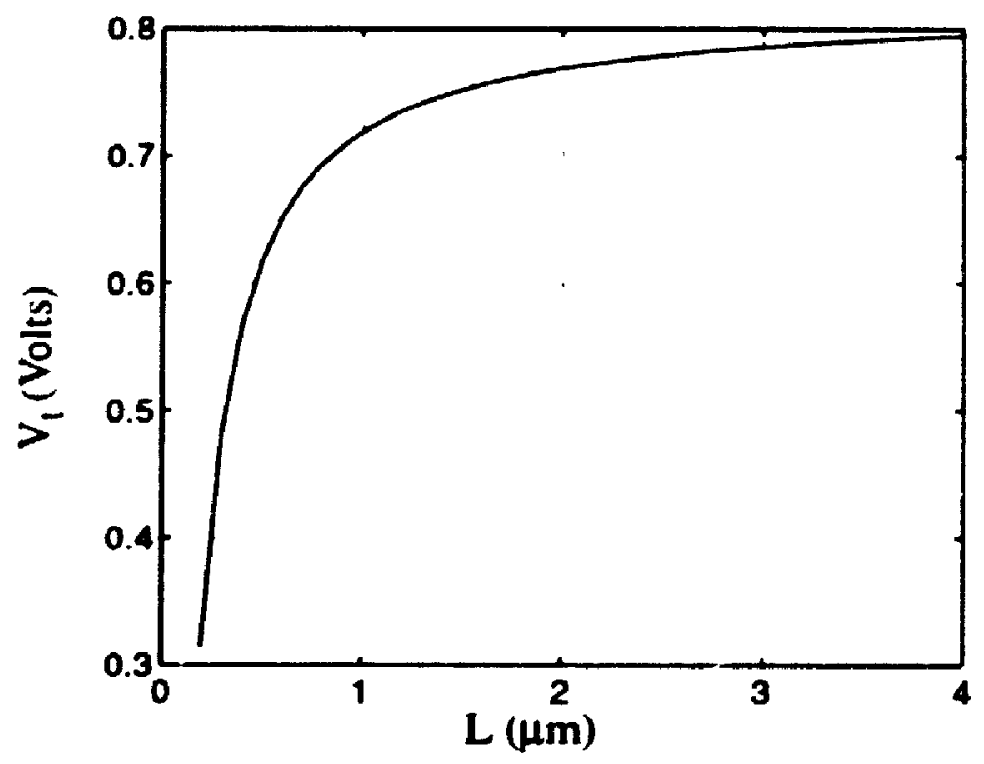

Figure 3.5: Threshold voltage as a function of channel length

MOSFET transistors with channel lengths shorter than the minimum feature size of a given technology (called "short-channel MOSFETs" hereafter), have lower threshold voltages due to SCE. These short-channel MOSFETs can be used as efficient analog 
switches [Bazarjani94a] due to higher $V_{\text {on }}$ (as a result of lower $V$, ) higher aspect ratio $(W / L)$ for the same width, and lower charge injection (based on channel length reduction). However, undesirable SCEs. such as increased subthreshold conduction and punchthrough current. set a limit to the minimum channel length acceptable for the shortchannel devices. Subthreshold conduction has been discussed before (section 2.2.2): in the following punchthrough is briefly discussed.

Short-channel MOSFETs are susceptible to barrier lowering that results in an unwanted current flow from drain to source. This phenomenon is affected by $L$ and "Ds. Figure 3.6a shows a turned-off nMOSFET switch which is not affected by punchthrough. For $v_{D S}=0$, the peak potential barrier is lowered if the channel length is not larger than the sum of the two depletion region widths (source and drain to substrate), as shown in Figure 3.6b. The barrier is lowered further for $v_{D S}>0$ (Figure 3.6c) because tield lines penetrate from drain to source [Troutman79]. The resulting punchthrough current has a iwo dimensional nature and no analytical model predicting it exist:. Two dimensional computer simulation and experiments show that punchthrough current decreases as $v_{D S}$ is lowered or negative bulk to source voltage is applied. In section 3.3, it will be shown that in a SC circuit all of the "off" switches have buth back bias and a maximum $v_{1, .}$ lower than $V_{D D^{\prime 2}}$, which helps in reducing punchthrough current.

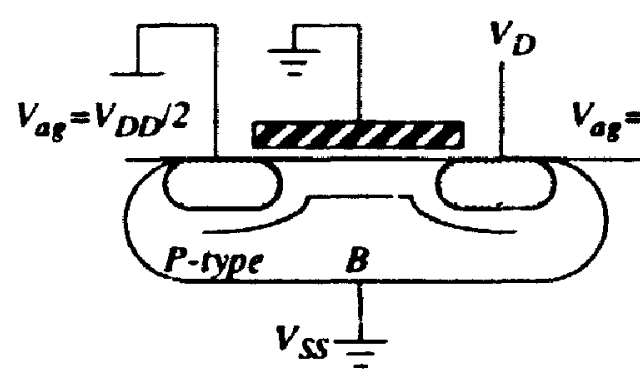

(a)

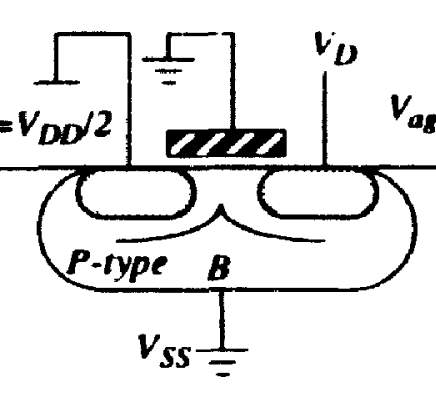

(b)

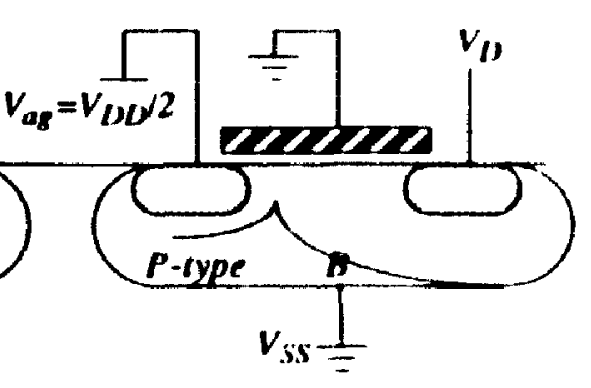

(c)

Figure 3.6: Turned-off nMOSFET switch exhibiting (a) no punchthrough, (b) punchthrough due to short channel length, and (c) punchthrough due to large drain to source potential

Channel length variation due to process fluctuation must be carefully considered for shonchannel MOSFETs. A drawn channel length $\left(L_{0}\right)$ can vary by " $\Delta L$ " due to error in 
lithography and etching. For channel lengths below the minimum feature size in a given technology, this variation might be larger. Thus, the optimum channel length must be selected such that devices with the absolute minimum channel length obtained on silicon have acceptable subthreshold and punchthrough currents.

\section{(II) Substrate Forward Biasing}

The threshold voltage of MOSFETs is a function of source to substrate voltage, owing to the body effect and is given by

$$
v_{1}=v_{10}+\eta\left(\sqrt{\phi_{B}+v_{S B}}-\sqrt{\phi_{B}}\right) .
$$

where $\gamma$ is the body effect coefficient defined as

$$
\gamma=\frac{\sqrt{2 q \varepsilon_{s i} N_{A}}}{C_{o x}} .
$$

Application of a negative $v_{S B}$ in nMOSFET (i.e. forward biasing the well potential with respect to source) will reduce the threshnld voltage. However, junction forward bias current limits the maximum forward biasing to about $0.4 \mathrm{~V}$.

Example 3.2: Assuming a $\gamma$ of $0.5 \mathrm{~V}^{1 / 2}$ and $\phi_{B}=0.8 \mathrm{~V}$, a forward bias voltage of $0.4 \mathrm{~V}$ reduces the threshold voltage by about $130 \mathrm{mV}$.

\section{Summary}

In a CMOS process with a normal $V_{1}$, lower threshold voltage MOSFETs are obtained using short-channel MOSFETs or by forward biasing the well.

\subsection{Low-V, Transmission Gate Design for SC Circuits}

In SC circuits, analog switches are implemented with MOSFET transistors having the following two distinct modes of operation.

"On" State:

In this case, MOSFETs operate in strong inversion-triode mode with a non-linear signal dependent on-conductance of approximately 


$$
g_{\text {on }}=2 \beta\left(v_{G S}-v_{t}\right) \text {. }
$$

where $\beta=(1 / 2)\left(\mu C_{o x} W / L\right)$.

“Off" State:

A turned-off MOSFET transistor $\left(v_{G}=0\right.$ ) operates in the weak inversion regime and it can be modelled as a current source with the following value:

$$
i_{\text {off }}=I_{D 0} \frac{W}{L} 10^{\frac{-\left(v_{s}+v_{t}\right)}{S}} .
$$

Here, $S$ is the subthreshold swing (the gate voltage change needed to reduce the leakage current by one decade), and $I_{D 0}$ is the value of the drain current when the gate to sour:e voltage of a unit transistor $(W / L=1)$ is set to $V$, and is given by

$$
I_{D 0}=\mu C_{o x} \phi_{t}^{2}
$$

An analog switch has several requirements, the most important ones being: low onresistance, full signal swing handling. and low off-current.

A low on-resistance switch is obtained by using low- $V$, MOSFETs. Rail-to-rall signal swing handling is achieved by using a full transmission gate (TG) switch also known as a complementary switch - an nMOSFET and a pMOSFET in parallel. Here, we focus on the behavior of the "off" switches in the context of stray-insensitive SC circuits.

Consider a stray-insensitive non-inverting $\mathrm{SC}$ integrator and its corresponding two-phase non-overlapping clock, as shown in Figure 3.7.

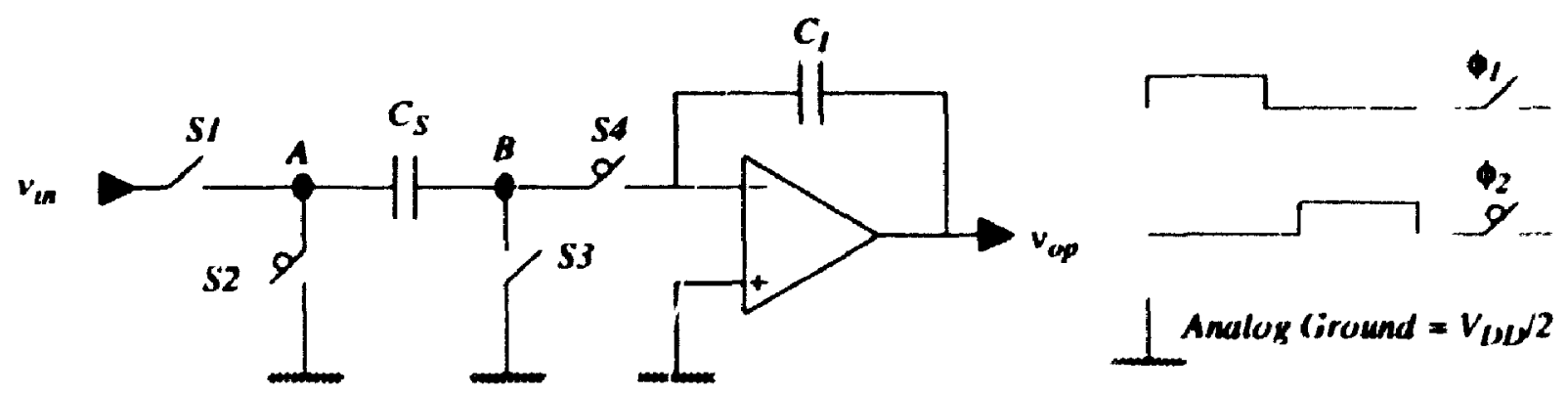

Figure 3.7: A non-inverting SC integrator 
All the "off" switches have the following properties:

- At least one side of any "off" switch is connected to analog ground, either directly or through another switch. This is a critical property used in the development of a nonleaky switch as described later.

- The maximum drain to source voltage across an "off" switch is less than $V_{D D} / 2$ and is given by

$$
\left|v_{D S}\right|_{\max }=\max \left\{\left.\left|\left(v_{D D^{\prime 2}}\right)-v_{i n}\right|_{\min }|\cdot| v_{i n}\right|_{\max }-\left(v_{D D^{\prime 2}}\right) \mid\right\} \text {. }
$$

Note that during off-state, the drain to source voltages of MOSFET switches $S 3$ and $S 4$ are almost zero. If the signal swing is limited to $v_{o n}<v_{i n}<V_{D D}-v_{o n}$, the maximum drain to source voltage of switches $S 1$ and $S 2$ will be

$$
\left|v_{D S}\right|_{\max }=\frac{v_{D D}}{2}-v_{o n}
$$

- There is a negative back bias (bulk to source voltage for nMOSFET) on all the switches. Again, assuming the signal swing is reduced by $v_{\text {on }}$ from each supply rail. the minimum back bias voltage on MOSFET switches $S 1$ and $S 2$ is

$$
v_{S B}=v_{o n} \text {. }
$$

Switches $S 3$ and $S 4$ have a back bias of $V_{D D} / 2$.

The effects of low- $V$, leaky MOSFET switches on the precision of analog SC circuits will be examined in the context of the non-inverting integrator illustrated in Figure 3.7. In this circuit, we assume that switch $S 1$ is implemented with a full transmission gate and all the other switches are simply nMOSFET transistors. In the following, error voltages on nodes $A$ and $B$ due to finite on-resistance and off-current are analyzed for different periods of a complete clock cycle.

\section{(I) During $\phi_{1}$}

The equivalent circuit of the non-inverting SC integrator (Figure 3.7) during $\phi_{1}$ is shown in Figure 3.8.

The leakage $i_{o f f 2}$ is the off-current through the nMOSFET switch $S 2$ and is calculated 


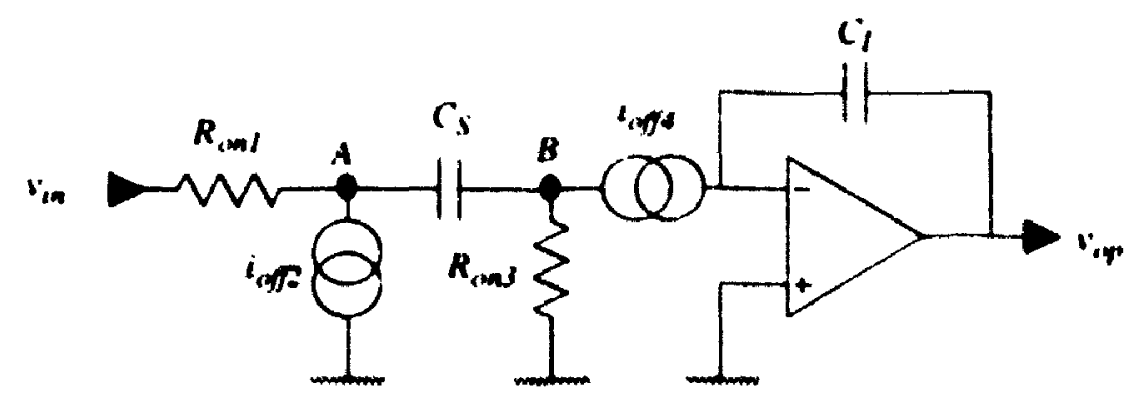

Figure 3.8: Equivalent circuit of Figure 3.7 during $\phi_{1}$

from (3-11) to be:

$$
i_{o f f 2}=I_{D 0} \frac{w}{L} 10^{-\left(\frac{v_{i n}+v_{i n}}{s}\right)}
$$

This off-current is largest for a signal level closest to $V_{S S}$ and causes an error voltage $\left(\Delta v_{1}\right)$ in the transmission of signal from $v_{\text {in }}$ to $v_{A}$ given by

$$
\Delta v_{1}=R_{\text {on } 1} i_{\text {off } 2} \text {. }
$$

where $R_{\text {on } 1}=1 / g_{\text {on } 1}$ is the effective on-resistance of toic complementary MOSFET switch $S 1$. The on-conductance $g_{\text {on } 1}$ is obtained from (3-10) as follows:

$$
\begin{aligned}
& B_{\text {ON1 }}=\left.\left.B_{\text {ONI }}\right|_{\text {MMOSFET }}{ }^{+B_{\text {ONI }}}\right|_{\text {pMOSFET }} \\
& \left.s_{O M 1}\right|_{\text {AMOSFET }}=2 \beta_{A}\left(v_{D D}-v_{\text {in }}-v_{t a}\right) \quad v_{i n}<v_{D D}-v_{I A} \\
& \left.B_{\text {on }}\right|_{\text {pMOSFET }}=2 \beta_{p}\left(v_{i n}-v_{t p}\right) \quad v_{i n}>v_{t p}
\end{aligned}
$$

The error voltage $\left(\Delta v_{1}\right)$ is a non-linear function of the input signal and causes offset and gain errors as well as distortion at the end of the cycle at the output. This error voltage is proportional to $\boldsymbol{R}_{o n 1}$ and $i_{\text {off } 2}$. $\boldsymbol{R}_{\text {on } 1}$ reaches a maximum at about mid-rail (assuming $\beta_{n}=\beta_{p}$ ) and $i_{o f f 2}$ rises as the signal gets closer to $v_{s s}$. Since $\Delta v_{1}$ is exponentially proportional to $i_{o f f 2}$, the maximum value of $\Delta v_{1}$ occurs when $i_{o f f 2}$ reaches its maximum value, i.e $v_{\text {in }}=V_{S S}$. Assuming $n M O S F E T s$ used in $S 1$ and $S 2$ have the same $W / L$, the maximum error voltage is 


$$
\left.\Delta v_{1}\right|_{\max }=\frac{\phi_{1}^{2}}{v_{D D}-v_{t}} 10^{-\frac{v_{1}}{s}}
$$

where $\phi_{\text {, }}$ is the thermal voltage $(k T / q=25 \mathrm{mV} @$ room temperature). This error voltage is independent of device size and is determined solely by the process technology parameters and the thermal voltage. For example, in a $1 \mathrm{~V}$ process with a threshold voltage of $200 \mathrm{mV}$ and a subthreshold swing of $100 \mathrm{mV} /$ decaric the maximum error voltage $\Delta v_{1}$ is about $7.8 \mu \mathrm{V}$.

A required dynamic range implies an upper limit on the error voltage, and from ( 3-21) one can find the minimum required threshold voltage. In the same way. for a given dynamic range and $\boldsymbol{K}_{\text {on }}$ the maximum tolerable $i_{\text {off }}$ can be found. For example, a $100 \mathrm{~dB}$ SNR with a power supply of $1 \mathrm{~V}$, and an on-resistance of $10 \mathrm{k} \Omega$ requires an off-current of less than $1 \mathrm{nA}$.

Switch $S 4$ is biased at mid-rail (analog ground and virtual ground); thus, ideally there is no potential across this switch and leakage off-current through it is zero. However. due to non-idealities (tinite opamp gain, etc.) there will be some voltage across switch $\mathrm{S4}$. Since this switcl. is biased at mid-rail, the subthreshold leakage is reduced by two di. "erent action:; threshold increase due to body effect, and negative gate to source voltage. The subthreshold leakage current will be

$$
i_{o f f 4}=I_{D 0} \frac{W}{L} 10^{-\left(\frac{V_{D D} / 2+V_{i}}{S}\right)}
$$

For some typical values $W / L=10, I_{D 0}=100 \mathrm{nA}$, and $V_{D D}=1 \mathrm{~V}$, a device with $V_{t}=180 \mathrm{mV}$ and $S=90 \mathrm{mV} /$ decade will have a leakage off-curtent of about $0.1 \mathrm{pA}$. which is very small and comparable to junction leakage.

\section{Non-Overlapping Period:}

The equivalent circuit of the SC integrator during the non-overlapping period is shown in Figure 3.9. As discussed in the previous section, the leakage off-currents through $S \geq$ and $\$ 4$ are negligible. 

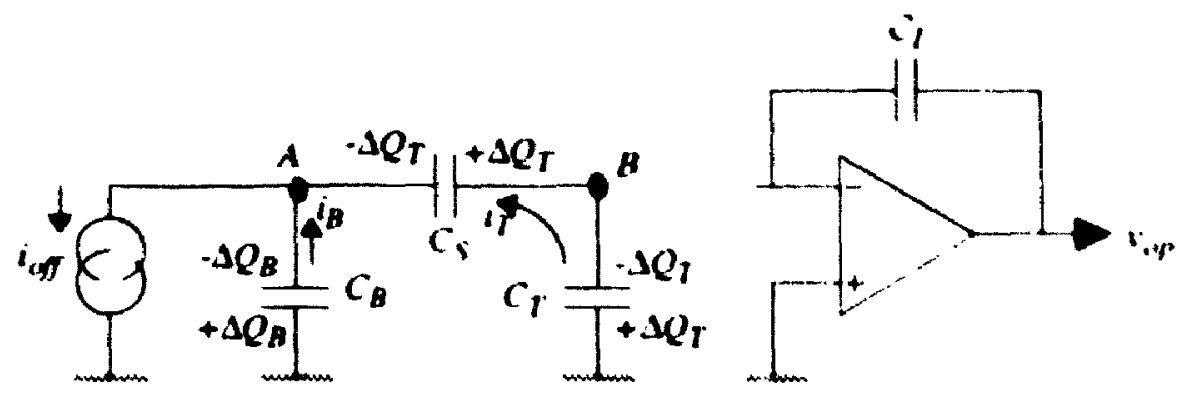

Figure 3.9: Equivalent circuit of Figure 3.7 during non-overlapping period

In this circuil., $C_{r}$ and $C_{B}$ are the parasitic sapacitances from the top and bottom plate of capacitor $C_{S}$ to ground. The sum of leakage currents through $S 1$ and 52 is called $i_{\text {"ff }}$. which is provided by $i_{T}$ and $i_{B}$.

These leakage currents cause a change in charge across each capacitor as shown in the above tigure. The error charge $\Delta Q_{B}$ across $C_{B}$ causes no net error at the output (this is a stray insensitive configuration), because Juring $\phi_{2}$ node $A$ is shoned to analog ground and $\Delta Z_{B}$ is dumped to ground. The error charges $\Delta Q_{T}$ on capacitors $C_{S}$ and $C_{j}$, have different polarities (during $\phi_{2}$ ) and as such cause no net error at the output.

\section{During $\phi_{2}$ :}

During $\phi_{2}$ the $S C$ integrator has an equivalent circuit as shown in Figure 3. III. At stemiy state. node $B$ will be at the virtua ground potential (similar to the negative input of the opamp). Thus, leakage $i_{\text {off } 3}$ would be uegligible. However, at the beginning of $\phi_{2}$. the voltage at node $B$ is changed suddenly from analog ground to $-v_{i n}$. This can cause switch $S 3$ to become leaky (for $v_{i n}$ close to $V_{D D}$ the source of $S 3$ will fall to $V_{S S}$ resulting in $\left.v_{G S}=0\right)$. Any charge lost by $S 3$ will cause an error voltage $\left(\Delta v_{2}\right)$ at the output given by

$$
\Delta v_{2}=\frac{1}{C_{S}} \int_{0}^{\delta t} i_{o f f} d !
$$

where $\delta t$ is the time taken for the opamp to force $V_{b}$ back to $\cdot$. irtual ground.

For example if $C=1 \mathrm{pF}$ and $\delta t=5 \mathrm{~ns}$, an average leakage current $i_{\text {off }}$ of 1 itA causes an error voltage of $\Delta V_{2}=5 \mu \mathrm{V}$.

Another source of error during this phase is due to leakage current $i_{\text {(ff } 1}$. Ideally, at the end 


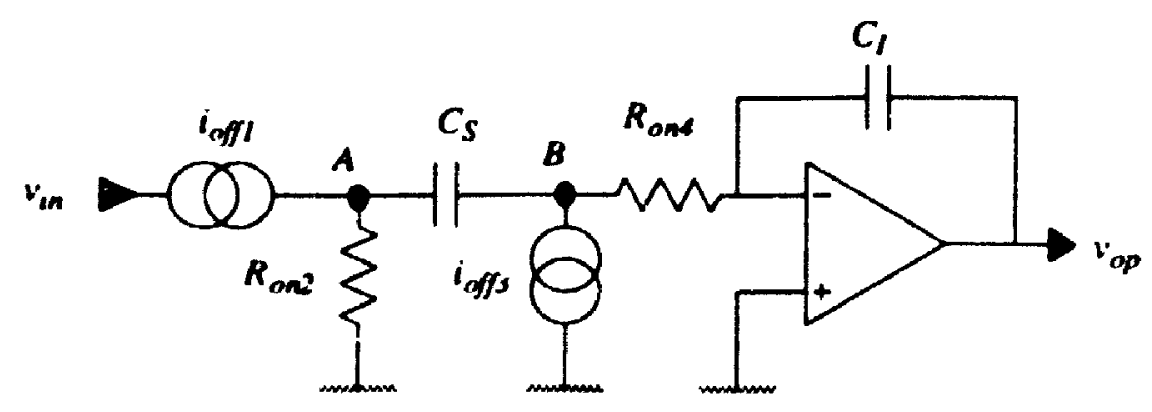

Figure 3.10: Equivalent circuit of Figure 3.7 during $\phi_{2}$

of $\delta_{2}$ node $A$ must be at the analog ground potential. Leakage current $i_{\text {off } 1}$ and onresistance $R_{\text {on } 2}$ cause an error voltage on node $A$ which is given by

$$
\Delta v_{3}=i_{\text {off } 1} R_{\text {on } 2}
$$

Assuming the input voltage is close to $V_{S S}$ and nMOSFET transistors in $S 1$ and $S 2$ have the same $W / L$, the error voltage is

$$
\Delta v_{3}=\frac{\phi_{1}^{2}}{\left(v_{D D^{\prime 2}}\right)-V_{1}} 10^{-\frac{v_{1}+v_{1 n}}{S}}
$$

Again. maximum error occurs for $v_{i n}$ close to $V_{S S}$ or $V_{D D}$. The maximum errur caused during this phase $\left(\phi_{2}\right)$ is larger than the maximum error caused during $\phi_{1},(3-21)$, because of the higher on-resistance in (3-24).

\section{Suinmary}

Low- $V$, MOSFETs are leaky and limit the accuracy of SC circuits. The error caused during saispling is process dependent. For a $1 \mathrm{~V}$ process with a threshold voltage of $100 \mathrm{mV}$ and a subthreshold swing of $100 \mathrm{mV}$, the sampling error is $156 \mu \mathrm{V}$ which limits the accuracy to about $76 \mathrm{~dB}$.

\subsection{Series Transmission Gate and Compcsite Switch}

As described in the previous section, subthreshold off-current through analog switches implemented with low threshold voltage MOSFETs introduces errors in SC circuits and reduces the dynamic range of the analog circuit. 
A method of reducing the off-current is by limiting the signal swing. If the signal swing is reduced by $\Delta V$ from each rail, the subthreshold off-current (3-11) will be reduced by $\Delta V / S$ decades. Notice that for a $\Delta V=200 \mathrm{mV}$ (which may be required for drain-source saturation voltage in the amplifier) and $S=90 \mathrm{mV} /$ decade. the off-current is reduced by more than 100 times.

Measurements of $i_{D S}$ vs. $v_{G S}$ for a $W / L=20 \mu \mathrm{m} / 0.5 \mu \mathrm{m}$ nMOSFET with a threshold voltage of $130 \mathrm{mV}$ are shown in Figure 3.11 for two different values of $v_{S A}=0$ and $0.2 \mathrm{~V}$. The drain to source voltage is set to $0.1 \mathrm{~V}$ in both measurements. Note that the

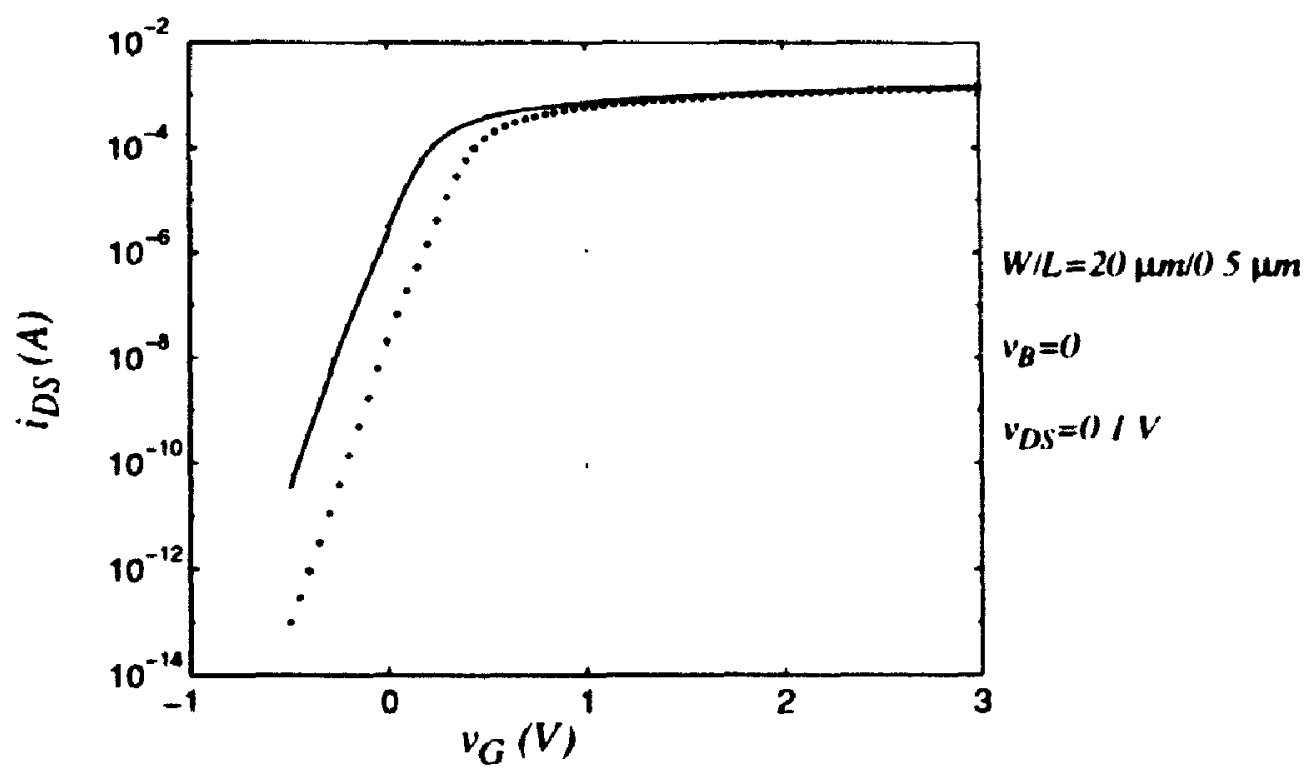

Figure 3.11: Measured $i_{D S}$ vs. $v_{G}$ for an nMOSFET with $V_{t}=130 \mathrm{mV}$ and $v_{S}=0$ (solid line) and $"_{s}=0.2 \mathrm{~V}$ (dotted line)

leakage off-current ( $i_{D S}$ at $V_{G}=0$ ) is reduced from $2.38 \mu \mathrm{A}$ to $20 \mathrm{nA}$ by applying a negative gate-to source voltage of $200 \mathrm{mV}$ (i.e., limiting the signal swing).

Another way of reducing the subthreshold off-current is by adjusting $V$, through back bias. A major problem with low- $V$, MOSFET transistors is the threshold voltage variation due to processing errors (about $\pm 100 \mathrm{mV}$ ) [Mizuno 94 I and temperature fluciuation (about $-1.25 \mathrm{mV} /{ }^{\circ} \mathrm{C}$ ). These can have a compounding effect and increase the off-current drastically. A technique of setting the $V_{l}$ to a constant value, by adjusting the substrate 
voltage. has been discussed in section 2.2 .2 .

In low-voltage design we can not afford to limit the signal swing by more than $150-200 \mathrm{mV}$, the minimum required drain-to-source saturation voltage in the amplifier. If the threshold voltage is very low, limiting the signal swing may not decrease the offcurrent enough and other techniques ought to be exploited.

\subsubsection{Series Transmission Gate Switch}

In section 3.3, it was shown that in a stray insensitive SC circuit "off" switches have one side connected to analog/virtual ground (either directly or through another switch). An nMOSFET switch during the off-phase is shown in Figure 3.12a.

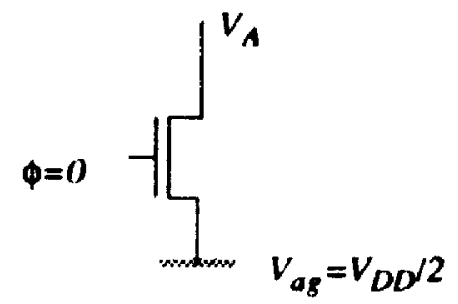

(a)

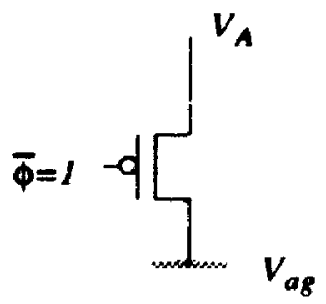

(b)

Figure 3.12: Turned-off (a) nMOSFET and (b) pMOSFET switches

For a $V_{A}>V_{D D} / 2$ the subthreshold leakage off-current through the nMOSFET switch is low because of the negative gate to source voltage and an increase in $V_{t}$ due to back bias. The $i_{\text {off }}$ current is,

$$
i_{o f f}=J_{D 0} \frac{W}{L} 10^{-P}
$$

where

$$
P=\frac{V_{D D} / 2+V_{t}}{S}
$$

However. as voltage $V_{A}$ drops below $V_{D D} / 2$, subthreshold leakage increases exponentially and reaches a maximum for $V_{A}=V_{S S}$. In this case the exponent $\left(P=V_{t} / S\right)$ is small for low- $V_{t}$ devices and results in a large off-current.

A turned-off pMOSFET switch (Figure 3.12b) has the opposite behavior; the off-current is 
low for $V_{A}$ below $V_{D D} / 2$ and the largest off-current flows for $V_{A}=V_{D D}$.

A very low leakage switch (with one side tied to analog ground), is obtained by a series transmission gate switch as shown in Figure 3.13. The maximum subthreshold leakage off-current through this switch is similar to an nMOSFET switch with $v_{A}=v_{D \mid l} / 2$, as analyzed previously.

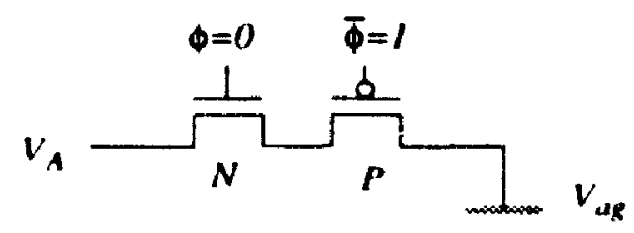

Figure 3.13: A series transmission gate switch

Figure 3.14 illustrates the simulated subthreshold leakage current through an nMOSFET and a series transmission gate (STG) switch. Threshold voltages of MOSFETs are sei $110 \mathrm{mV}$ and transistor sizes are $(W / L)_{p}=2(W / L)_{n}=10$.

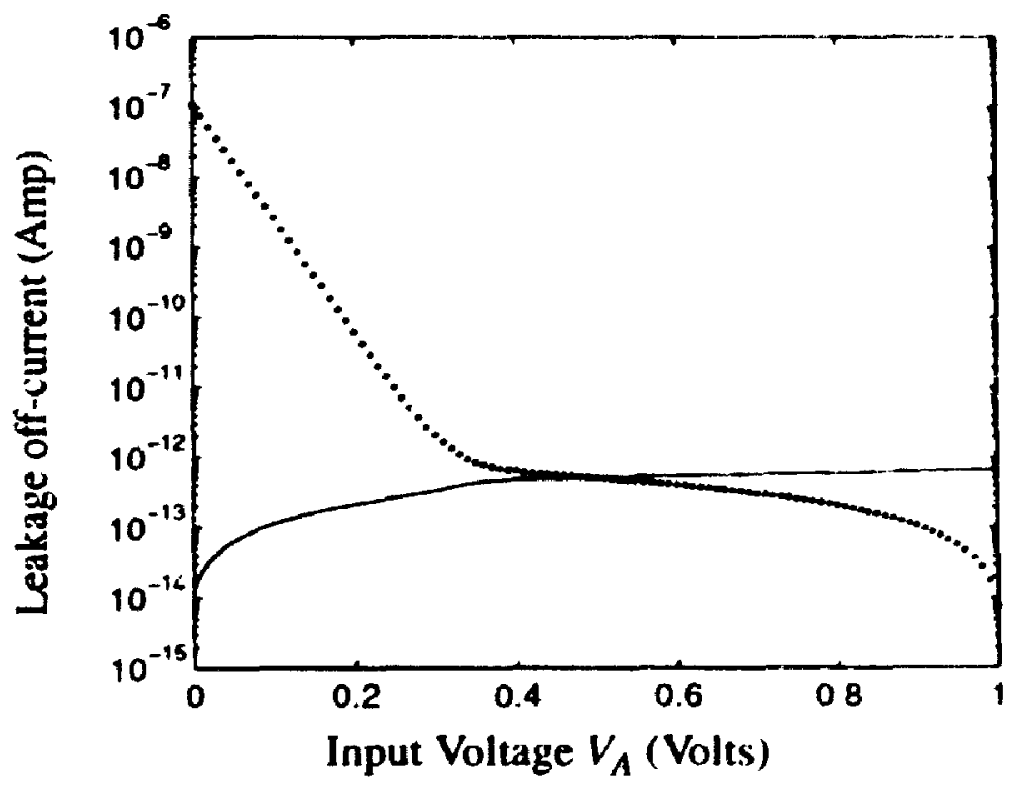

Figure 3.14: Lewage off-current through an nMOSFET switch (dotted line) and a STG switch (solid line) versus input voltage

The leakage off-current through the STG switch is very low $(\sim 1 \mathrm{pA})$ while a low- $V$, nMOSFET has a relativeiy high leakage off-current $(\sim 0.1 \mu \mathrm{A})$ for low input voitages. 
The on-conductance of the series transmission gate switch is

$$
g_{o n}=\frac{2 \beta_{n}\left(V_{D D}-V_{i n}-V_{i n}\right) \beta_{p}\left(V_{i n}-V_{t p}\right)}{\beta_{n}\left(V_{D D}-V_{i n}-V_{i n}\right)+\beta_{p}\left(V_{i n}-V_{t p}\right)}
$$

For $\beta_{n}=\beta_{p}$ and $V_{t n}=V_{t p}=V_{t}$, the maximum on-conductance for a STG occurs at $V_{L, D} / 2$. This is exactly where all the switches to analog ground are operating. The maximum on-conductance of a series transmission gate is

$$
\left.g_{\text {on }(\max )}\right|_{S T G}=\beta\left(\frac{V_{D D}}{2}-V_{t}\right)
$$

which is lower than the on-conductance of a simple nMOSFET by a factor of 2 . Simulation for on-conductance, Figure 3.15, confirms the validity of the above analysis.

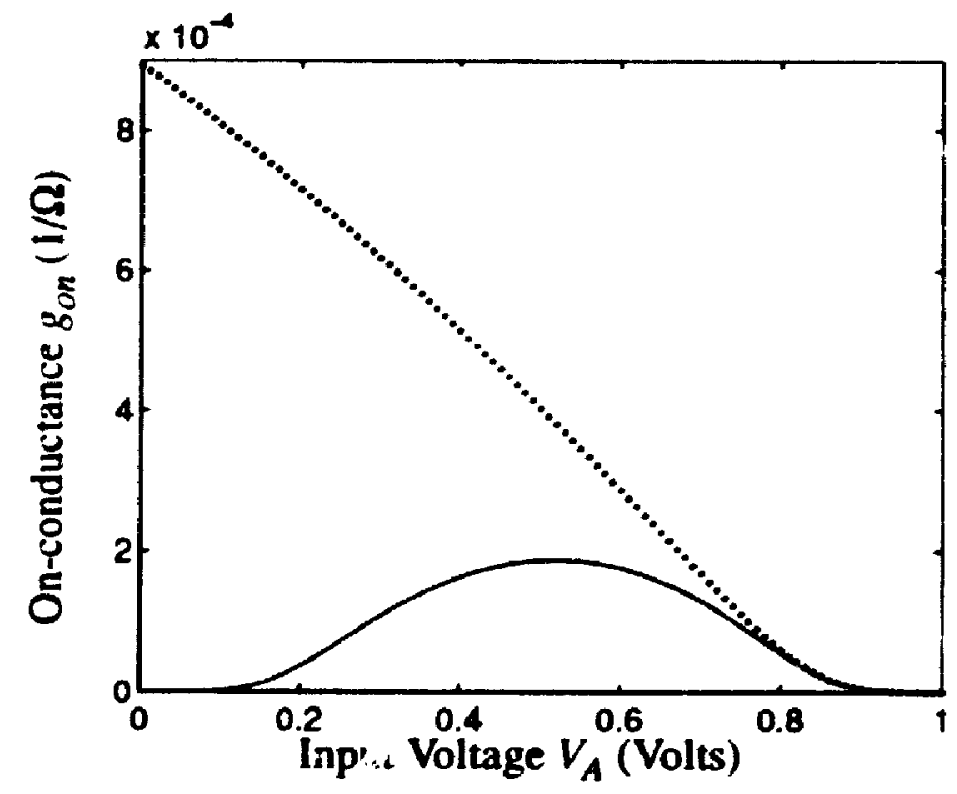

Figure 3.15: On-conductance of the nMOSFET (dotted-line) and STG switch (solid-line) versus input signal

A drawback of the series transmission gate switch is the limited input signal swing. This switch cannot conduct signals that are within a $V$, of the supply rails. In the next section. a solution to this problem is presented.

\subsubsection{Composite Switch}

In a multi-threshold process, the high- $V$, (threshold adjusted) transistors can $b$. Ised in a 
parallel transmission gate (TG) configuration along with the series transmission gate switch to handle rail-to-rail switch capability. The composite switch is shown in Figure 3.16 (the $V$, adjusted MOSFETs are shown with bold lines in the channels).

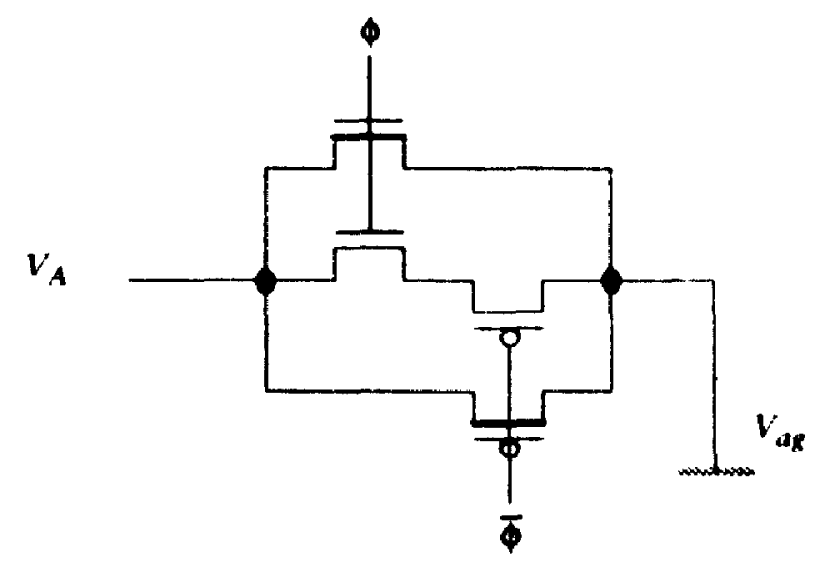

Figure 3.16: A composite switch

The simulated on-conductance of the composite switch is shown in Figure 3.17. In this

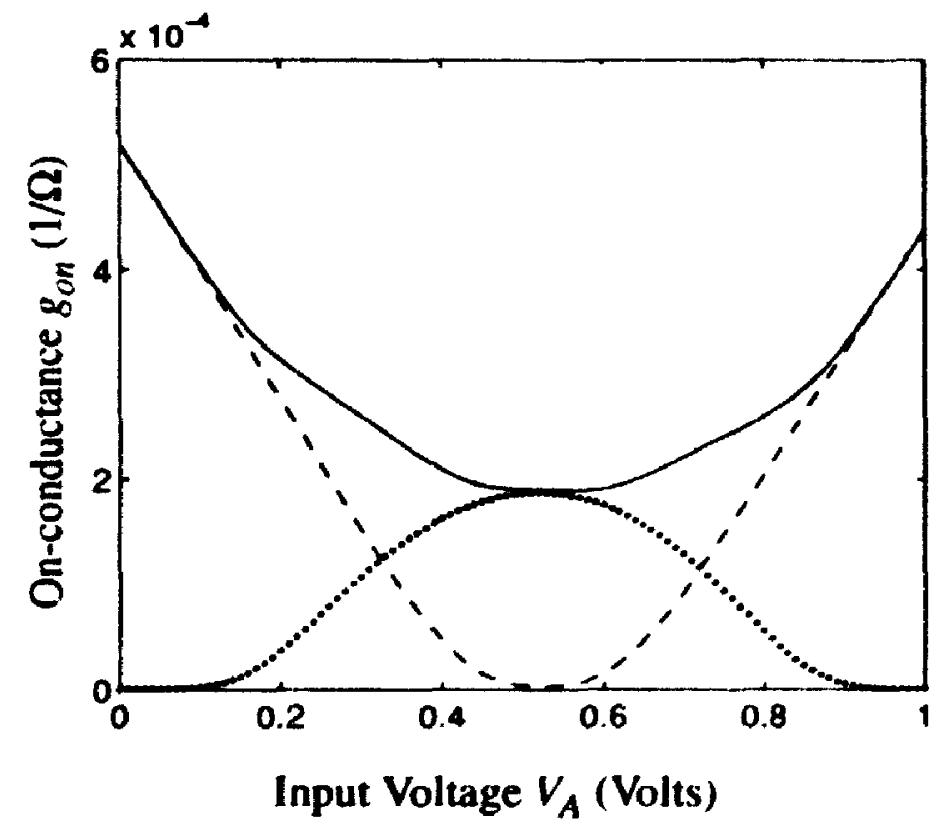

Figure 3.17: Simulated on-conductance for high- $V_{1}$ TG switch (dashed-line). low- $V_{t}$ series transmission gate switch (dotted-line), and the composite switch (solid-line) versus input signal

configuration. the high- $V$, parallel transmission gate conducts well when the signal is 
close to the rails, and the low- $V$, series transmission gate conducts well when the signal is at mid-rail. The on-conductance and leakage through this composite switch is simulated along with simple high- $V$, and low- $V$, parallel transmission gates and all three are illustrated in Figure 3.18 and Figure 3.19.

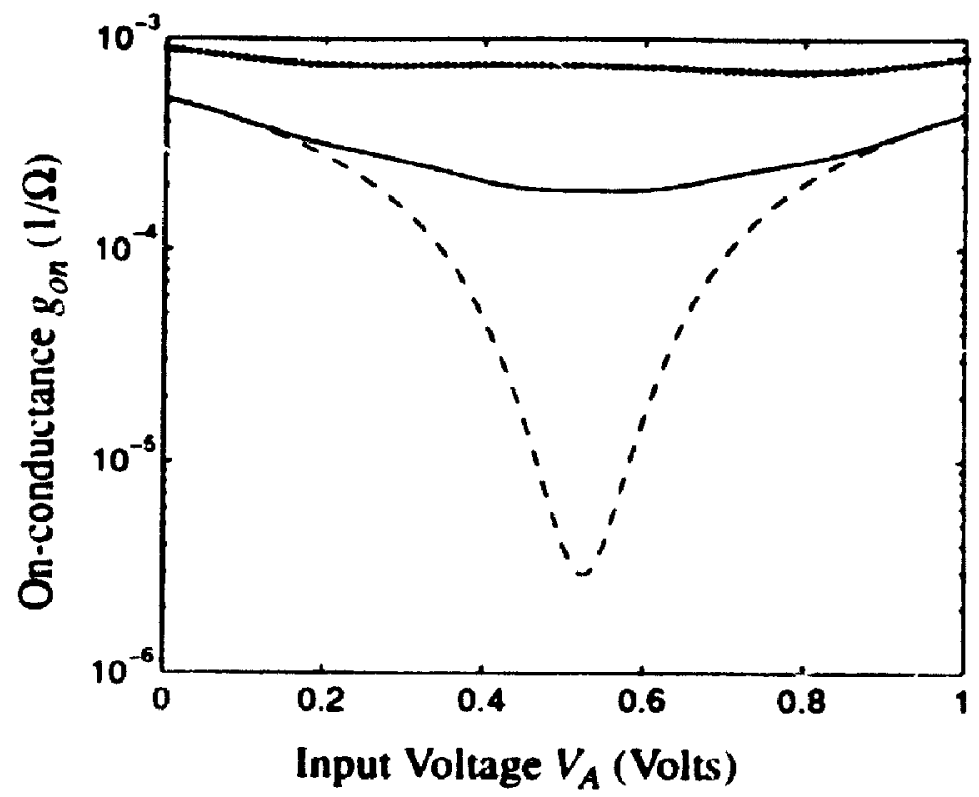

Figure 3.18: On-conductance for high- $V$, TG switch (dashed-line), low-V, TG switch (dotted-line), and the composite switch (solid-line) versus input signal

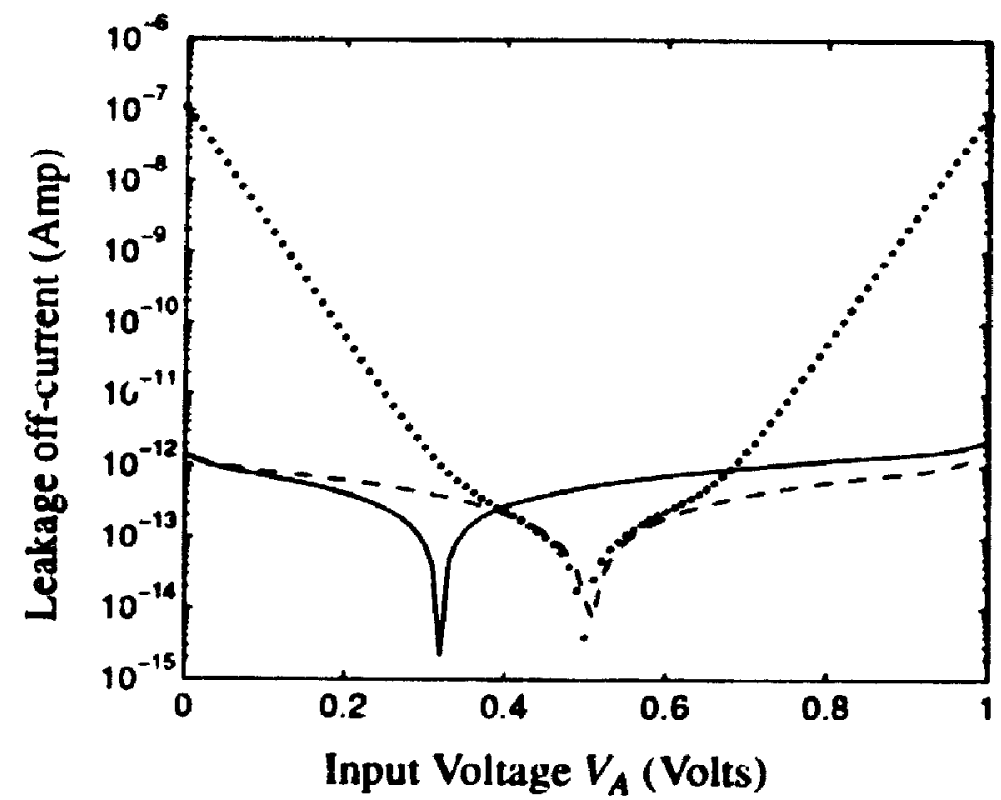

Figure 3.19: Leakage : urrent for high- $V$, TG switch (dashed-line), low- $V$, TG switch (dotted-line), and the composite switch (solid-line) versus input signal 


\section{Summary}

Subthreshold off-current through MOSFET switches is reduced by (1) limiting the signal swing. (2) adjusting the $v$, by back bias. and (3) using low-leakage series transmission gate and composite switch.

\subsection{A 2.25 V Second-Order Sigma-Delta Modulator}

This $2.25 \mathrm{~V}$ modulator is based on an existing $3.3 \mathrm{~V}$ SC oversampled double integration sigma-delta modulator [Howlett93] in BATMOS, Northern Telecom's $1.8 \mu \mathrm{m}$ BiCMOS process [Hadaway91]. The $3.3 \mathrm{~V}$ design operates at $2 \mathrm{MHz}$ and achieves $94 \mathrm{~dB}$ SNDR for an oversampling ratio of 256 . Simulations indicated that the characteristic of the amplifier and comparator blocks used in this modulator are not signiticantly degraded at $2.25 \mathrm{~V}$. The modulator performance was limited by the increase in switch resistance at $2.25 \mathrm{~V}$. This has been remedied by using short-channel MOSFET switches. At $2.25 \mathrm{~V}$ the SNDR of the original design drops to $86 \mathrm{~dB}$. However, the moditied design using $0.6 \mu \mathrm{m}$ channel length MOSFET switches achieves $92 \mathrm{~dB}$ of SNDR at $2.25 \mathrm{~V}$.

Unfortunately, a two-stage BiCMOS opamp is used in this modulator which can not operate below $2.25 \mathrm{~V}$. Thus, in-circuit low-voltage performance of these short-channel switches can not be verified below $2.25 \mathrm{~V}$.

The block diagram of this modulator is similar to the one in |Boser8x| and it is a differential SC design, implemented using two single-ended opamps.

\subsubsection{Short-Channet MOSFETs}

Medici. a 2-D device simulator, was used to find the minimum achievable channel length for short-channel MOSFETs with acceptable puachthrough current in BATMOS. Measurements were also carried out on different sizes of short-channel MOSFETs to verify the simulation results and MOSFETs with channel length of $0.6 \mu \mathrm{m}$ were found reliable as low-voltage switches.

Characteristics of different short-channel MOSFETs (all with $W / L=11 \mu \mathrm{m} / 0.6 \mu \mathrm{m}$ ) were measured for both nMOSFE $r$ and pMOSFET transistors. At supply voltage of $3 \mathrm{~V}$. the on-resistances of switches biased at mid-rail $\left(v_{S B}=1.5 \mathrm{~V}\right.$ and $\left.v_{D B}=1.6 \mathrm{~V}\right)$ were measured. Results are shown in Table 3.1. 


\begin{tabular}{|c|c|c|c|}
\hline \multirow{2}{*}{$\begin{array}{c}\text { Transistor } \\
\text { Type }\end{array}$} & \multicolumn{2}{|c|}{$R_{\text {un }}$} & \multirow{2}{*}{ Reduction } \\
\cline { 2 - 4 } & $L=0.8 \mu \mathrm{m}$ & $L=0.6 \mu \mathrm{m}$ & \\
\hline nMOSFET & $1.3 \mathrm{~K}$ & $0.709 \mathrm{~K}$ & $45 \%$ \\
\hline pMOSFET & $5.8 \mathrm{~K}$ & $3.2 \mathrm{~K}$ & $44 \%$ \\
\hline
\end{tabular}

Table 3.1: On-resistance of switches with $W=10 \mu \mathrm{m}$

Further measurements of the $0.6 \mu \mathrm{m}$ nMOSFET switch show that $R_{o n}$ of $1.2 \mathrm{k} \Omega$ is achieved with a supply voltage of $2.1 \mathrm{~V}$.

Figure 3.20 illustrates the $i_{D S}$ versus $v_{G S}$ characteristic of an rMMOSFET with $0.6 \mu \mathrm{m}$ channel length under two different $v_{D S}$ conditions.

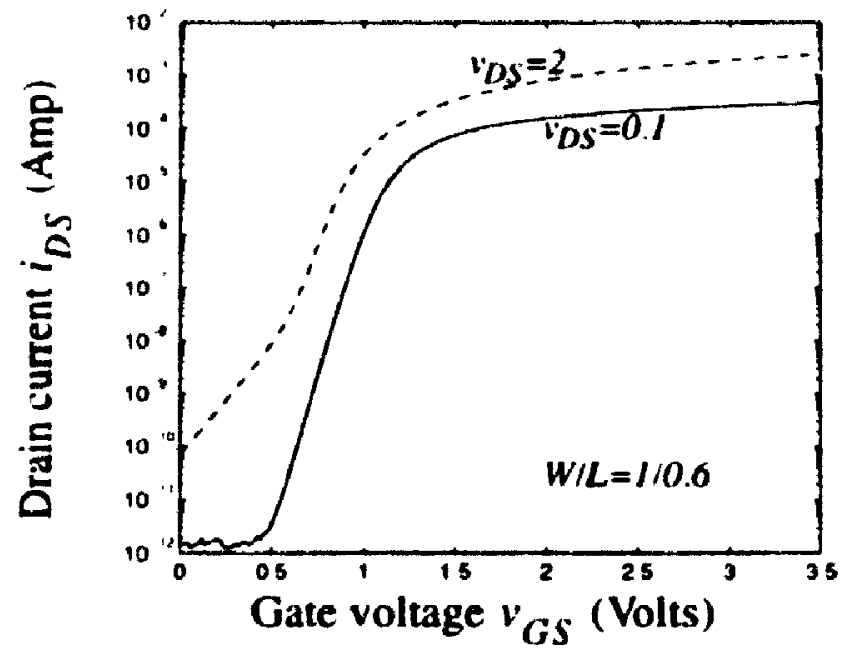

Figure 3.21): Drain current versus gate voltage for a $0.6 \mu \mathrm{m}$ nMOSFET with $v_{D S}=0 . I \mathrm{~V}$ (solid line) and $v_{D S}=2 \mathrm{~V}$ (dashed line)

Since the turn-off leakage ( $i_{D S}$ at $v_{G}=0$ ) is of prime importance to $r, i_{D S}$ versus $v_{D S}$ is also measured for the same device (a $0.6 \mu \mathrm{m}$ nMOSFET), with different back bias voltages. It is seen (Figure 3.21) that extremely low current leaka $z$ is obtained by setting the back bias to $0.5 \mathrm{~V}$. Both Figure 3.20 and Figure 3.21 show current per micron of width of the transistor.

Measurements of a $0.6 \mu \mathrm{m}$ pMOSFET device gave similar results. 


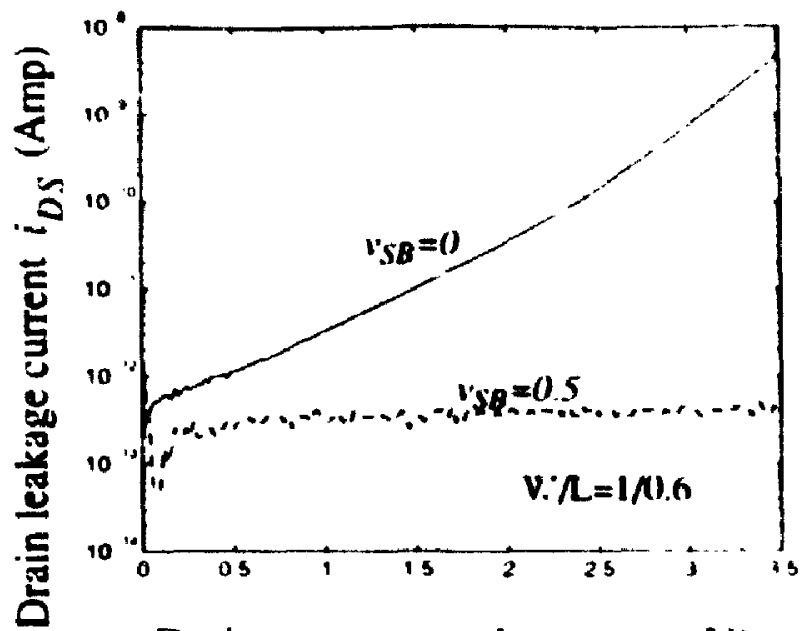

Drain to source voltage v'p.S (V)

Figure 3.21: Drain off-current $\left(1_{(j}=0\right)$ versus drain to source voltage for a $(1.6 \mu \mathrm{m}$ $n$ MOSFET with $v_{S B}=0$ (solid line) and $v_{S B}=0.5 \mathrm{~V}$ (dashed line)

\subsubsection{Short-Channel MOSFETs Layout Issues}

Channel length variation due to processing errors (lithography and etching) is very critical for short-channel MOSFETs and must be minimized. Some layout techniques that help to reduce the $3 \sigma$ (inter die and intra wafer) channel length variations are as follows.

- Place the structure of interest close to the center of the die: thereby ensuring the best possible optical and focus performance.

- Maintain symmetry around the structure of interest to ensure similar resist How/ planarization from all directions [Gehm92].

- Keep all the underlying steps (e.g., device well edges) as far away from the area of interest as is feasible.

\subsubsection{Measured SNDR}

The second-order $\Sigma \Delta$ modulator using short-channel MOSFET switches was fabricated in BATMOS and the measured SNDR is shown in Figure 3.22. Zero dB input level corresponds to a $1.5 \mathrm{~V}$ peak-to-peak signal. This modulator operates at $2.25 \mathrm{~V}$ and has at maximum SNDR of $92 \mathrm{~dB}$ for an oversampling ratio of 256 . The SNDR of the urigunal $3.3 \mathrm{~V}$ design drops to $86 \mathrm{~dB}$ at $2.25 \mathrm{~V}$ supply voltage. 


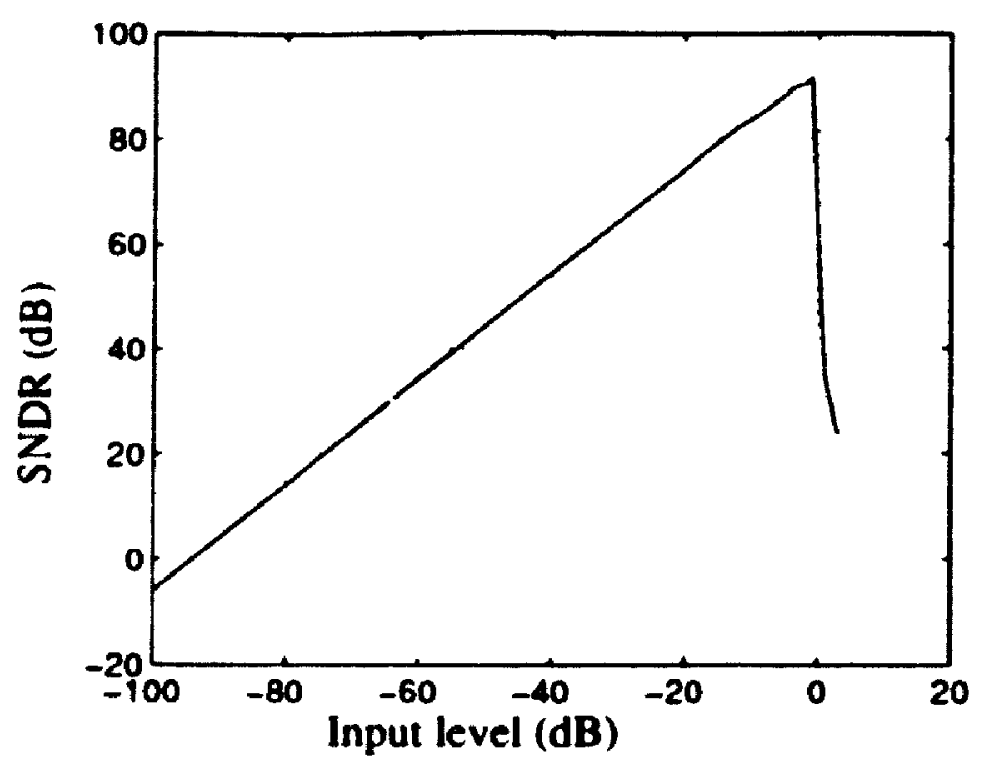

Figure 3.22: Measured $\Sigma \Delta$ modulator performance

\subsection{A 1 V First-Order Sigma-Delta Modulator}

In a low supply-voltage environment, fully differential circuits are preferred due to larger dynamic range. elimination of second harmonic distortions, and the tolerance to various common mode noises. This design was done at the early stages of process development and the CAD tools had very limited capability. Thus, a simple single-ended first-order SC $\Sigma \Delta$ modulator was chosen as a benchmark to demonstrate the feasibility of using natural MOSFETs for $1 \mathrm{~V}$ SC design. To our knowledge, this is the first reported SC $\Sigma \Delta$ modulator operating at a power supply voltage of $1 \pm 0.1 \mathrm{~V}$ [Bazarjani95a].

The architecture of a tirst-order SC $\Sigma \Delta$ modulator is shown in Figure 3.23. It consists of a discrete integrator and a two level quantizer in a negative feedback loop.

This modulator was implemented in a $0.5 \mu \mathrm{m} n+/ p+$ dual poly gate CMCS process using natural threshold voltage MOSFETs. The natural transistors in this process have theshold voltages of about $2(x) \mathrm{mV}$ which is suitable for $1 \mathrm{~V}$ circuits. In the following, design of IV opamp. comparator and switches used in this circuit is discussed along with some simulation and measured results. 


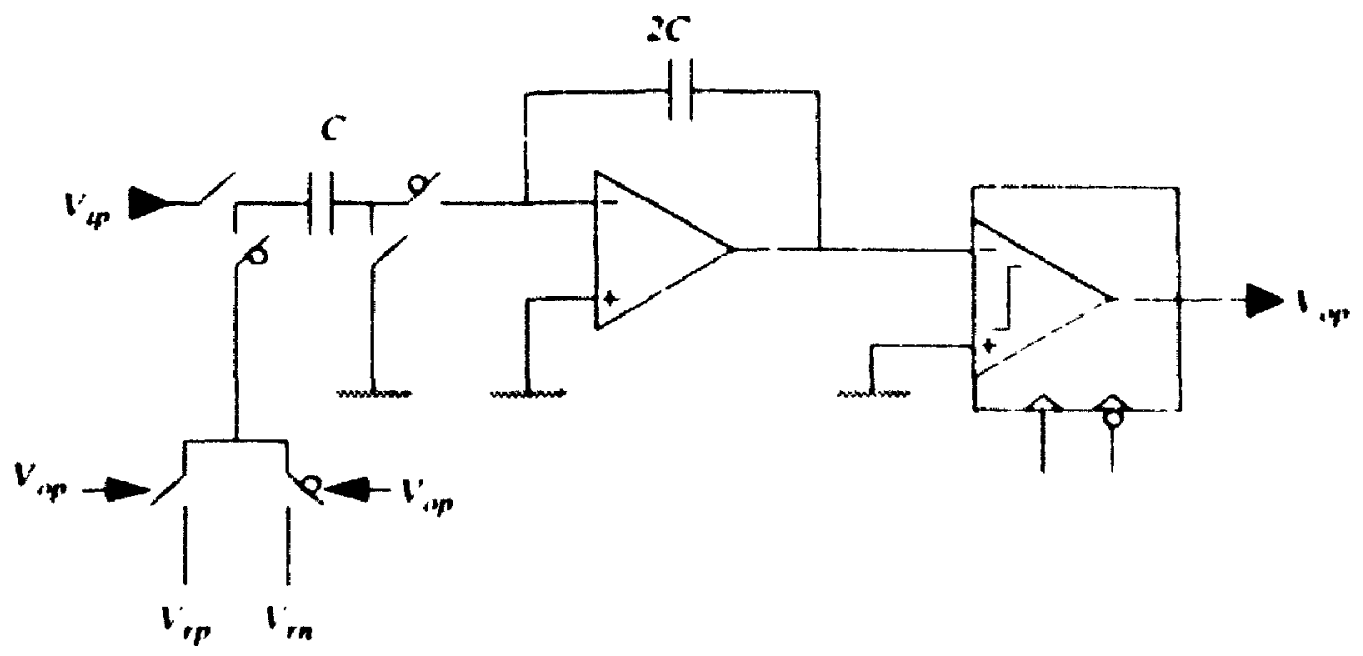

Figure 3.23: A first-order SC $\mathrm{SA}$ modulator

\subsubsection{V opamp}

Lowering the power supply voltage directly reduces the dynamic range alod consequently the accuracy of the analog circuitry. Thus rail-to-rail signal swing is desired to muximize the dynamic range. In a SC circuit, signals at the inputs of the opamp are at analog ground. thus no input signal swing is required. However, the output(s) of the opamp must provide the largest possible signal swing. Each transistor at the output stage requires a certain voltage to be kept in saturation $\left(v_{D S}>v_{(i S}-v_{f}\right)$ which limits the output signal swing. Therefore, cascode structures are avoided at the output stage of the amplitier. A Iw(1)-slage pole-splitting opamp. Figure 3.24, provides enough gain and maximum signal swing at the output of the amplifier.

In this opamp. the bias current $I_{\text {bias }}$ is se to $10 \mu A$ and the compensation capacitor $f^{\circ}$, is 2 pF. The dimensions of all the MOSFETs are listed in Table 3.2.

\begin{tabular}{|c|c|c|c|c|c|c|c|c|c|c|}
\hline MOSFET & $N I$ & $N 2$ & $N 3$ & N4 & $P I$ & $P 2$ & $P 3$ & $P 4$ & $P 5$ & $P 0$ \\
\hline \hline$W(\mu \mathrm{m})$ & 10 & 10 & 10 & 2 & 120 & 120 & 60 & 60 & 4 & 60 \\
\hline$L(\mu \mathrm{m})$ & $I$ & $I$ & $I$ & $l$ & 0.5 & 0.5 & 1 & 1 & 1 & 1 \\
\hline
\end{tabular}

Table 3.2: Dimension of MOSFETs used in the opamp of Figure 3.24

This opamp was simulated in Eldo [Eldo] using MISNAN, a physically baxed MOSFET 


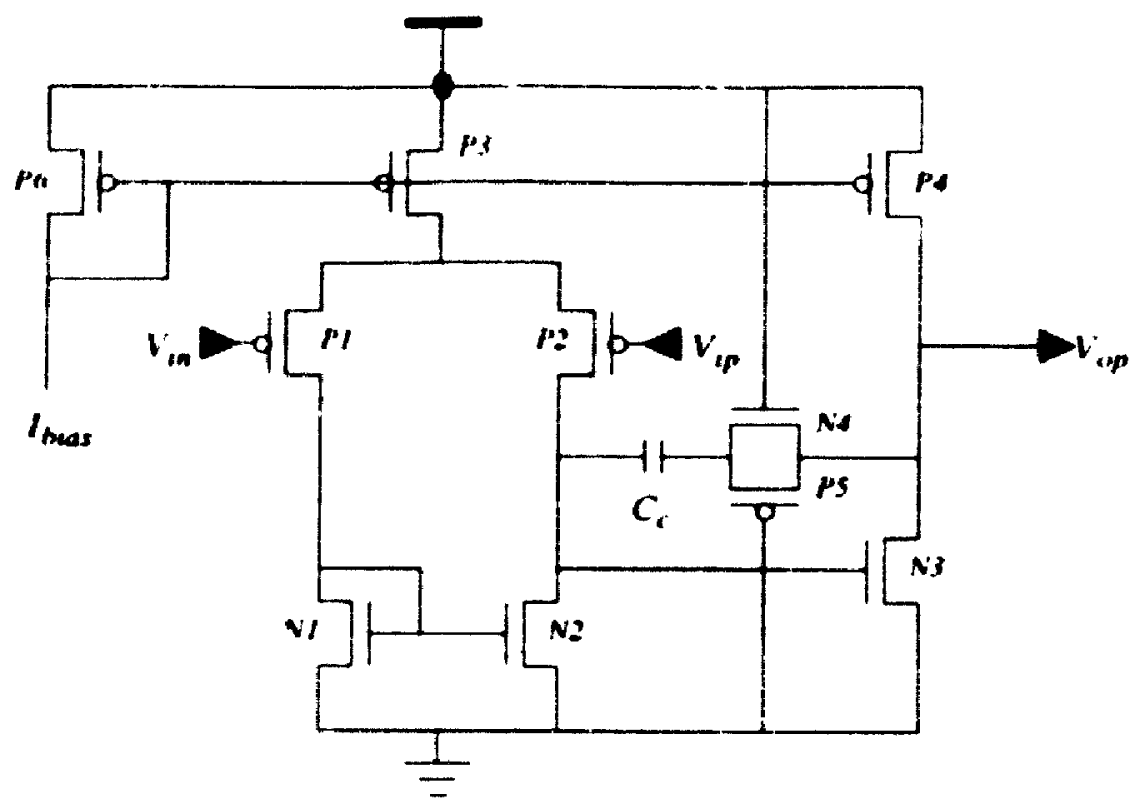

Figure 3.24: A two-stage pole-splitting opamp

model [Boothroyd92]. The device model parameters were obtained from [Tarry5]. where parameters are estimated using SUPREM3 and MINIMOS5 simulations.

The overall simulated opamp performance at $1 \mathrm{~V}$ power supply voltage and $1 \mathrm{pF}$ load is summarized in Table 3.3.

\begin{tabular}{|c|c|}
\hline Parameter & Result \\
\hline \hline DC gain & $60 \mathrm{~dB}$ \\
\hline Unity gain bandwidth & $40 \mathrm{MHz}$ \\
\hline Phase Margin & $60^{\circ}$ \\
\hline Slew rate & $10 \mathrm{~V} / \mu \mathrm{s}$ \\
\hline Output range & $6 . .5 \mathrm{~V}$ \\
\hline Power Dissipation & $30 \mu \mathrm{W}$ \\
\hline
\end{tabular}

Table 3.3: Simulated opamp performance for a 1 pF load capaciti: ace

\subsubsection{Comparator}

In $\Sigma \Delta$ modulators the performance of the comparator is not tritical. A class AB comparator similar to [Boser88] is used here. Figure 3.25 illustrates the schematic of this 
comparator. Simulations indicate a delay of $6 \mathrm{~ns}$ is achieved at a $1 \mathrm{~V}$ power supply tor a 1 pF load capacitance.

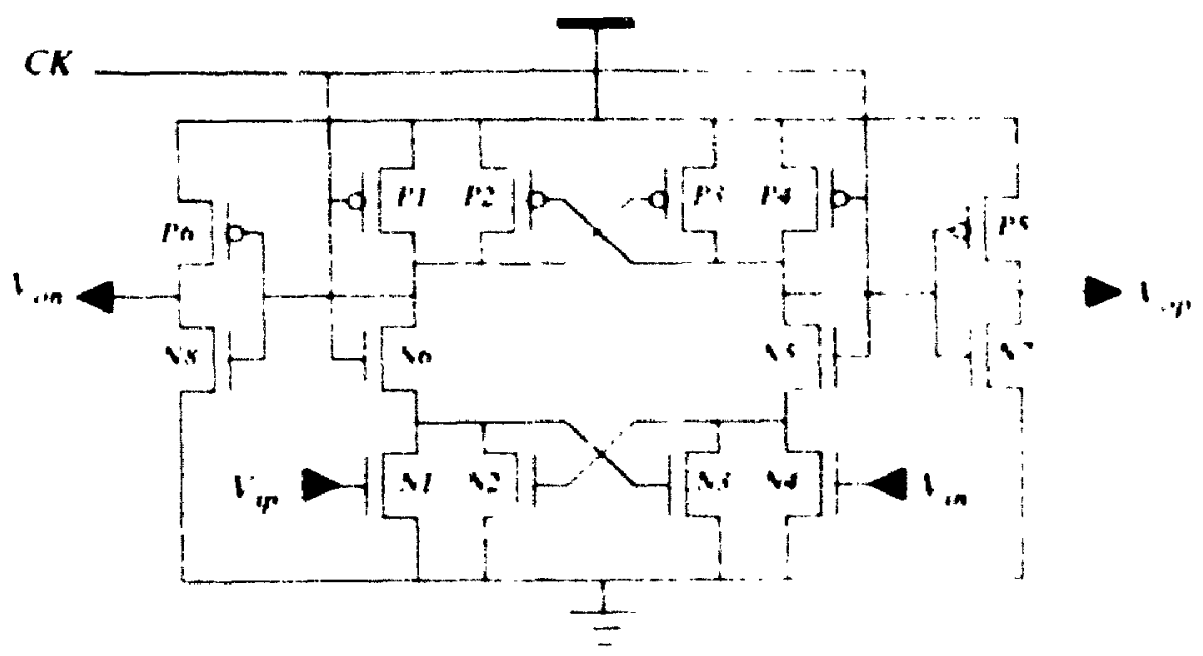

Figure 3.25: Sichematic of the cinmparatur

All the transistors sizes for this comparator are listed in Table 3.4

\begin{tabular}{|c|c|c|c|c|c|c|c|c|c|c|c|c|c|c|}
\hline MOSFET & NI & $N_{2}$ &.$v_{1}$ & $N 4$ & NS & vo & N7 & NA & $P I$ & $r 2$ & ri & P & $1 \times 4$ & 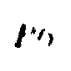 \\
\hline$W(\mu m)$ & 10 & 10 & 10 & 10 & 5 & 5 & 10 & $10 \mathrm{i}$ & 1 & (1) & (1) & i & 101 & 16 \\
\hline L (um) & 2 & 2 & I & $?$ & 0.5 & 0.5 & 0.5 & 0.5 & $0 x$ & $\therefore$ & $\therefore$ & (i) & 11 & ;ים \\
\hline
\end{tabular}

Table 3.4: Transister sizes for the comparator

\subsubsection{Switches}

As previously discussed. a good analog switch will exhibit a low on-resistame: nten turned on and a very low off-cursent when turned off. In this SC design. iwlaches ase implemented with complementary low $-V_{t}$ natural MOSFETs. A transmuslsu gate suith with $W / L=1.5 \mu \mathrm{m} /(1.5 \mu \mathrm{m}$ for both $\mathrm{nMOSFET}$ and pMOSFET was smuiated in Filo at $1 \mathrm{~V}$ supply voltage. Figure 3.26 shows the on-resistance of the .. alnsmissull gatie switc . a funciion of the input signal level.

A worst cave on-resistance of $12 \mathrm{k} \Omega$ is obtanned for a signal levei of $7(4) \mathrm{mV}$ in the is circuit of Figure 3.23 (w') of these switches and the "amping capacitur con tutute an $k($ carcuit. For the sampling capacitor of $1 \mathrm{pF}$ the time coastu!" $\tau$ will the 24 11, set:ling to 

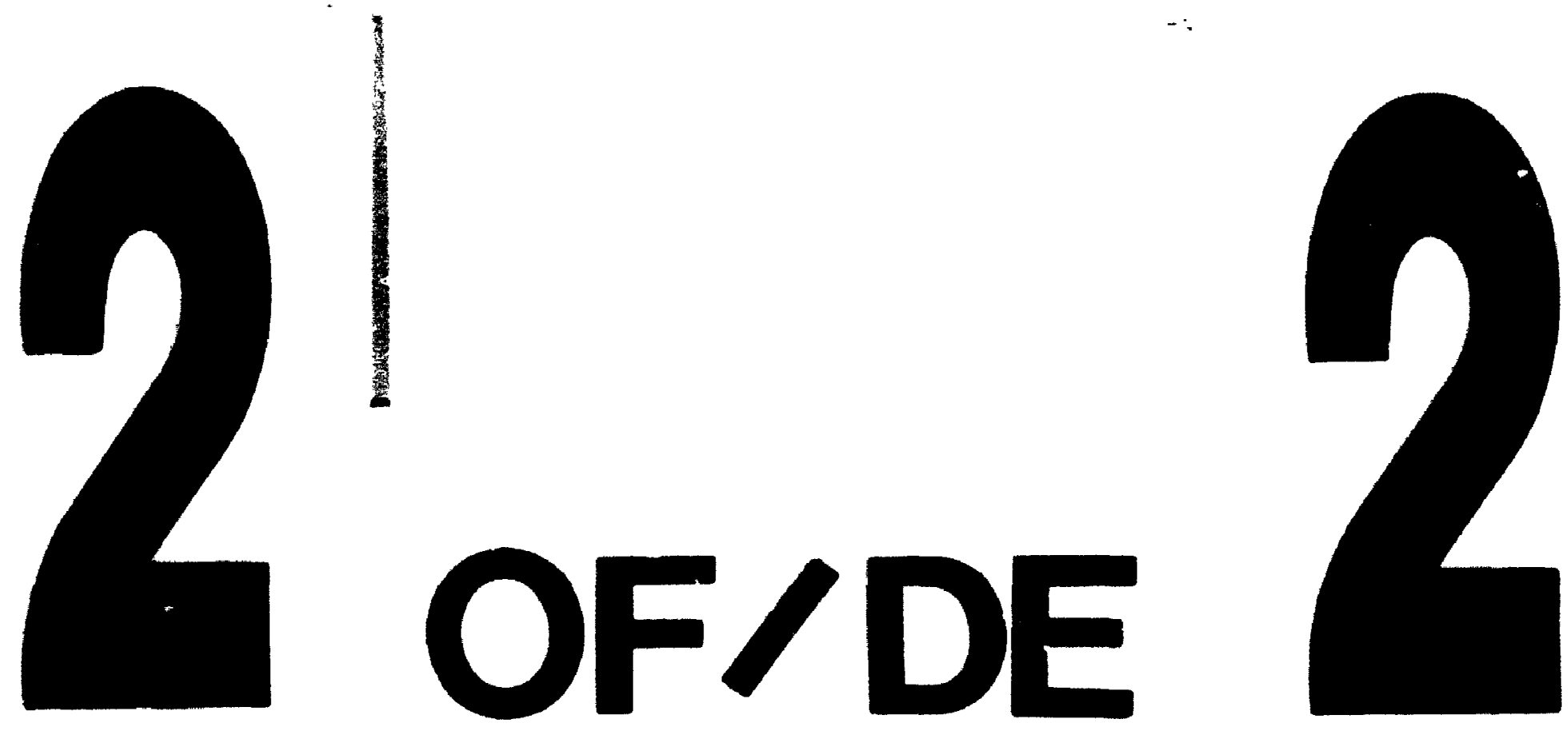

PM-1 31/" "X4" PHOTOCRAPHIC MICROCOPY TARCET Mas 1010a ANSH/SO w2 EOUTMLEMT

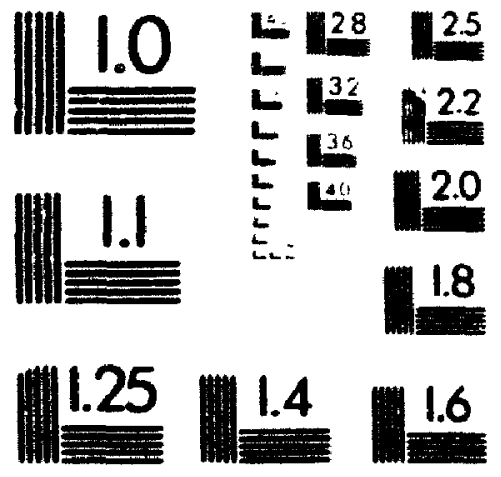

PRECHELONam RESOLUTION TARGETS 


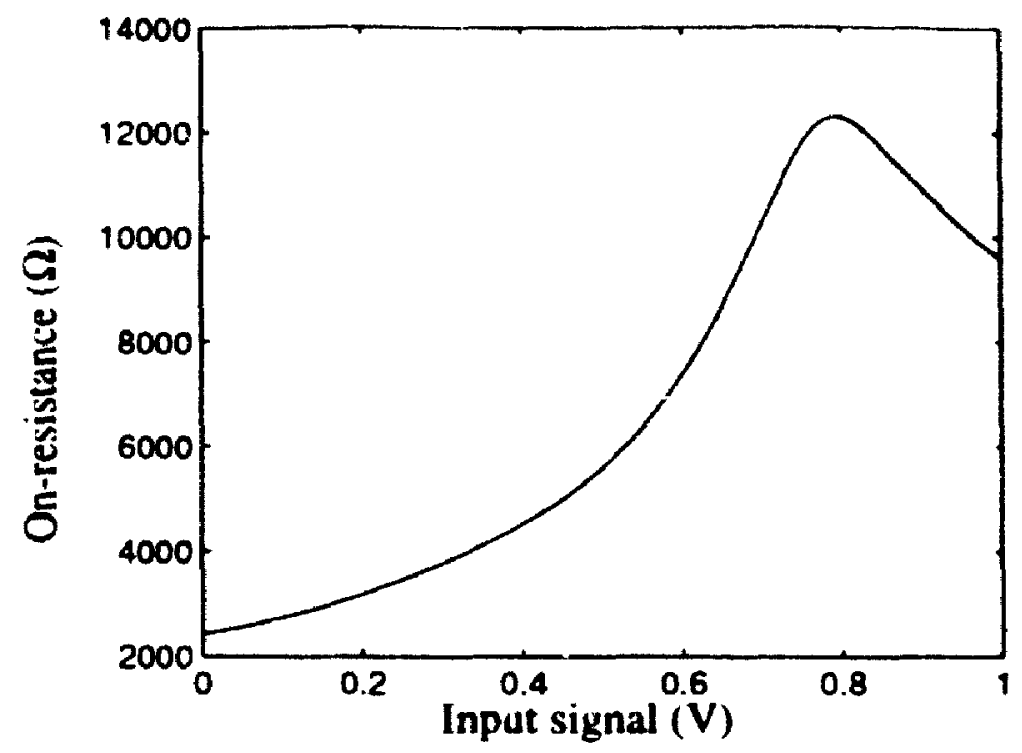

Figure 3.26: Simulated transmission gate switch resistance as a function of input signal

$0.1 \%$ of the full scale requires $168 \mathrm{~ns}(7 \tau)$ allowing an upper limit of $3 \mathrm{MHz}$ operation for this switch.

The subthreshold off-current of these switches can be calculated from the simplitied equation of (3-11). In this process, typical values of $I_{D 0}$ and $S$ for an nMOSFET are $100 \mathrm{nA}$ and $86 \mathrm{mV} /$ decade respectively. The $W / L=1.5 \mu \mathrm{m} / 0.5 \mu \mathrm{m} n \mathrm{nMOSFET}$ switch will have less than $1 \mathrm{nA}$ subthreshold leakage current for a signal at $V_{s s}$. However, the minimum signal in this $\Sigma \Delta$ modulator is about $0.25 \mathrm{~V}$. Therefore. the maximum leakage current through the above nMOSFET switch (when the signal is $0.25 \mathrm{~V}$ ) is less than $10 \mathrm{pA}$. Simulation of the leakage current through a natural nMOSFET transistor is shown in Figure 3.27 and the results correlate well with the above analysis.

\subsubsection{Measured Results for the 1 V Sigma-Delta Modulator}

The modulator was fabricated in a $0.5 \mu \mathrm{m} \mathrm{n}+\mathrm{p}^{+}$dual poly gate CMOS process with a linear poly-poly capacitor and triple level metal CMOS process. The chip die photograph is shown in Figure 3.28.

The modulator was tested at a clock frequency of $1 \mathrm{MHz}$ using a $1 \mathrm{~V}$ supply voltage and reference voltages of $\pm 0.25 \mathrm{~V}$ (with respect to analog ground, $0.5 \mathrm{~V}$ ). The output 


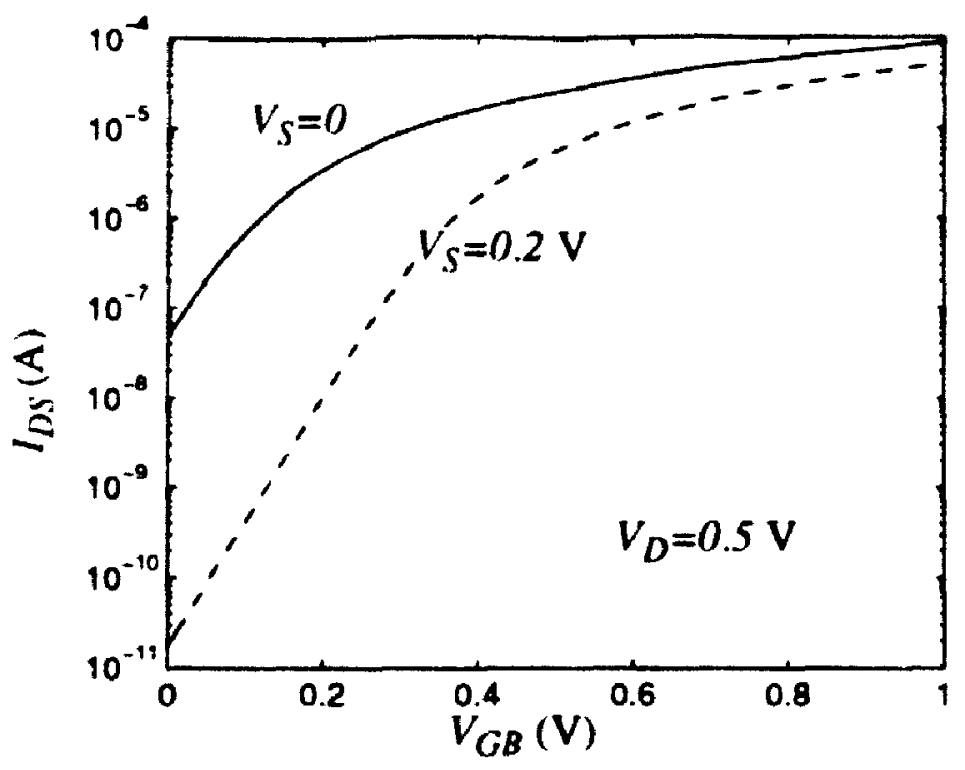

Figure 3.27: Simulated off-current for the nMOSFET switch

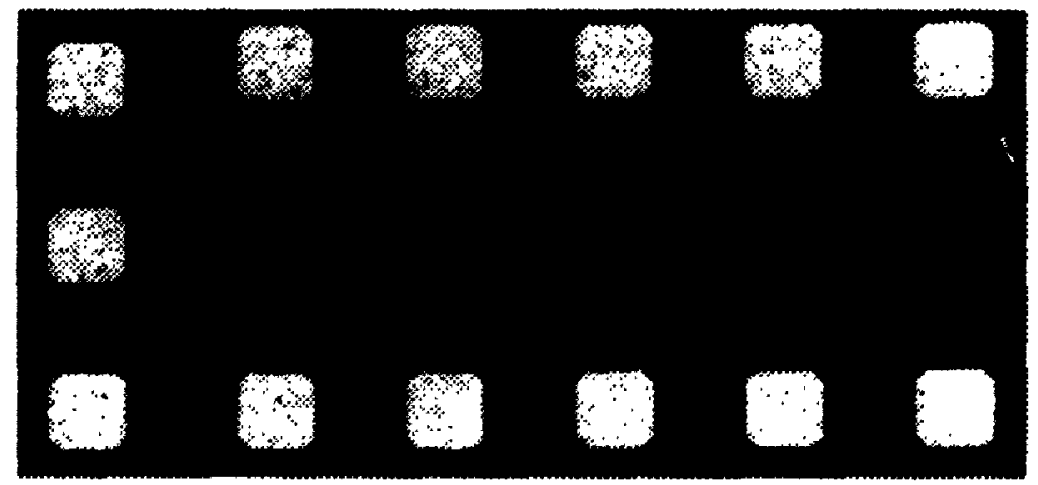

Figure 3.28: Chip die microphotograph

spectrum for an input signal of $\mathbf{4} \mathbf{k H z}$ and $420 \mathrm{mVpp}$ is shown in Figure 3.29 .

The output bit-stream was captured by a logic analyzer and 131072-point FFT was performed in Matlab [Matlab]. For an oversampling of 128, a SNDR of $54 \mathrm{~dB}$ is obtained.

\subsection{Summary}

In this chapter, a process technology and two circuit techniques for achieving low- $V_{t}$ MOSFETs in current CMOS processes were presented. Effects of low- $V_{t}$ subthreshold 


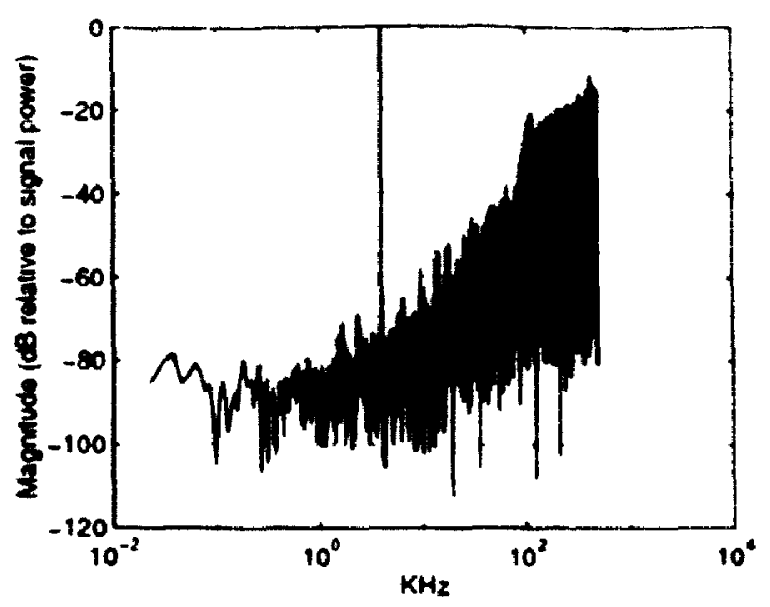

Figure 3.29: Measured spectrum ( Vip $=420 \mathrm{mVpp}$ )

leakage currents to the precision of analog SC circuits were analyzed. It was shown that off-current leakage through MOSFET switches reduces the accuracy of SC circuits.

Methods of reducing the leakage current through analog switches were discussed and two very low leakage analog switches suitable for SC circuits were proposed.

Finally, two experimental low-voltage voice-band $\Sigma \Delta$ modulators using low- $V$, MOSFETs were described. In the first design. an existing $3.3 \mathrm{~V} \Sigma \Delta$ modulator designed in $0.8 \mu \mathrm{m}$ BiCMOS process was modified using "short-channel MOSFET" as switches and the new design operates at $2.25 \mathrm{~V}$. The second design is a $1 \mathrm{VSC} \Sigma \Delta$ modulator using low- $V$, natural MOSFETs in a $0.5 \mu \mathrm{m}$ CMOS technology. 


\section{Chapter 4}

\section{High-Speed SC Fourth-Order Bandpass Sigma-Delta Modulators}

In Chapter 2, a method of transfer function design for a bandpass $\Sigma \Delta$ modulator from a lowpass $\Sigma \Delta$ modulator using a $z^{-1} \rightarrow-z^{-2}$ change of variable was discussed. It was also mentioned that the stability and SNR characteristics of the resulting bandpass $\Sigma \Delta$ modulator are identical to those of the lowpass prototype.

This chapter starts by introducing a $z$-domain architecture for a fourth-order bandpass $\Sigma \Delta$ modulator. This modulator is obtained by performing the above mapping to a secondorder (double integration) lowpass $\mathbf{\Sigma} \Delta$ modulator. The resulting bandpass modulator is a double-resonator $\Sigma \Delta$ modulator. In the sampled-data domain. an efficient method of implementing resonators uses two delay cells in a negative feedback loop. Switchedcapacitor delay circuits are considered next and a double-sampled SC delay cell is presented. The impacts of non-ideal circuit behaviors on the performance of a simple SC delay cell and the double-sampled SC delay circuit are analyzed. Then, two SC implementations of the fourth-order bandpass $\Sigma \Delta$ modulator-namely a simple SC structure and a double-sampled SC architecture-are presented along with Eldo simulation results. Finally, the design of both modulators in a $0.5 \mu \mathrm{m}$ CMOS process is considered and measured results of the modulators are presented.

\subsection{Modulator Architecture}

The loop filter transfer function for a fourth-order bandpass $\Sigma \Delta$ modulator was derived in Chapter 2, and is 


$$
u_{h p^{4}}(z)=\frac{z^{-4}+2 z^{-2}}{\left(1+z^{-2}\right)^{2}}
$$

Assuming the quantization error $e$ to be white noise and the comparator gain to the unity. the output-input transfer characteristic of this modulator is

$$
Y(z)=z^{-4} X(z)+\left(1+z^{-2}\right)^{2} E(z)
$$

Lowpass $\Sigma \Delta$ modulators are usually implemented as a cascade of integrators. A natural choice for bandpass modulators seems to be a casiade of resonators. One such architecture is shown in Figure 4.1. The quantizer is typically irnplemented using a 1-bit comparator

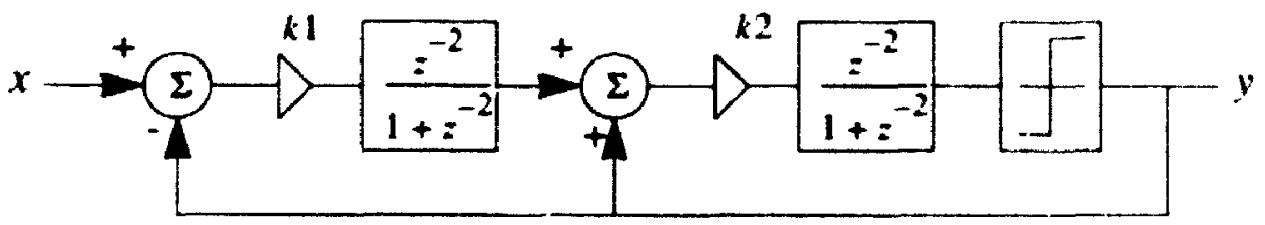

Figure 4.1: A fourth-order double resonator bandpass $\Sigma \Delta$ modulator

with two levels at $\pm V_{\text {ref }}$. The $z$-domain transfer function of this modulator is

$$
\left[1+(2-k 2) z^{-2}+(1+k 1 k z-k 2) z^{-4}\right] Y(z)=k 1 k 2 z^{-1} x(z)+\left(1+z^{-2}\right)^{2} E(z) \text {. }
$$

Comparing equations (4-2) and (4-3), the values of $k 1$ and $k 2$ are tound to be 0.5 and 2 respectively. Since the second resonator is followed by a high gain quantizer, the coefticient $k 2$ is irrelevant [Boser88] and is set to 0.5 , as is the coefficient of the first stage. This archilecture is a direct map of the lowpass $\Sigma \Delta$ modulator in [Boser8X] to bandpass by transforming integrators to resonators. Analogous to the lowpass prototype. the signal swings at the output of the resonators are almost within the full scale input range, $\pm V_{\text {ref }}$. Figure 4.2 illustrates the histograms of the signal levels at the output of the resonators for an input signal of $6 \mathrm{~dB}$ below full scale (i.e., the peak-to-peak signal amplitude is $V_{\text {ref }}$ ). We use statistical techniques to characterize signal swings, beciause sigma-delta modulators produce pseudo-random outputs.

The noise transfer function of this modulator has a pair of complex conjugate zeros 


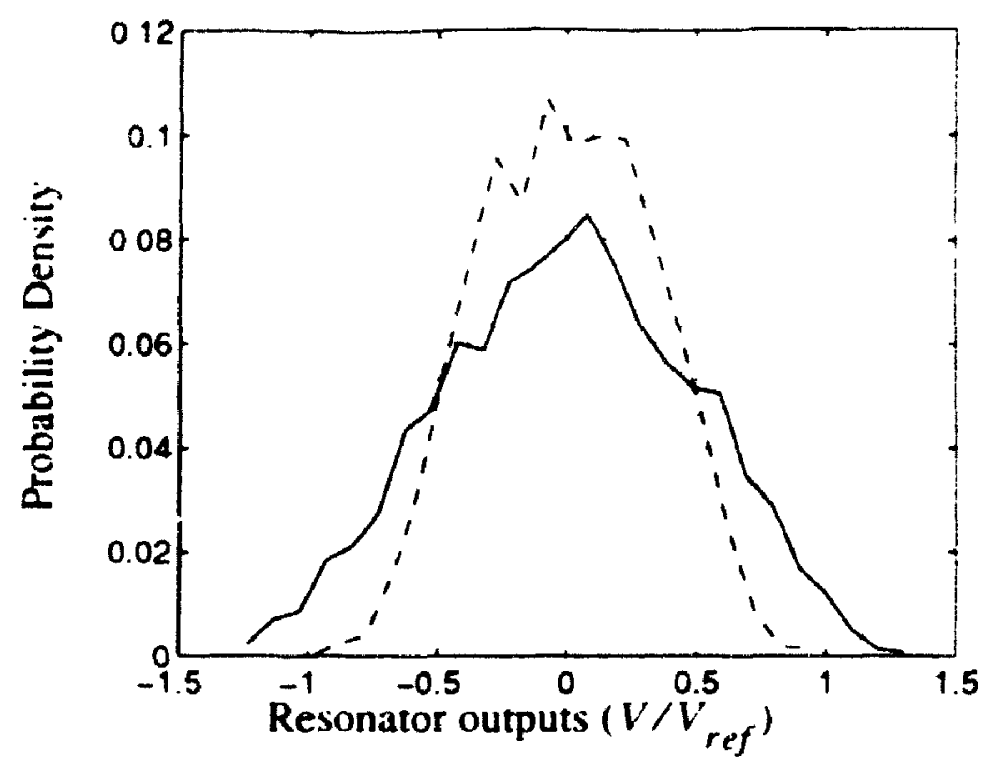

Figure 4.2: Histograms of output levels for the first (solid line) and the second (dashed line) resonator for a $-6 \mathrm{~dB}$ tone input

located at $z= \pm j$. In the frequency domain, this corresponds to notches around $(2 n+1) f_{s} / 4$, where $n=0,1,2, \ldots$, and $f_{s}$ is the sampling frequency. The $z$-domain simulated output spectrum of this modulator is shown in Figure 4.3.

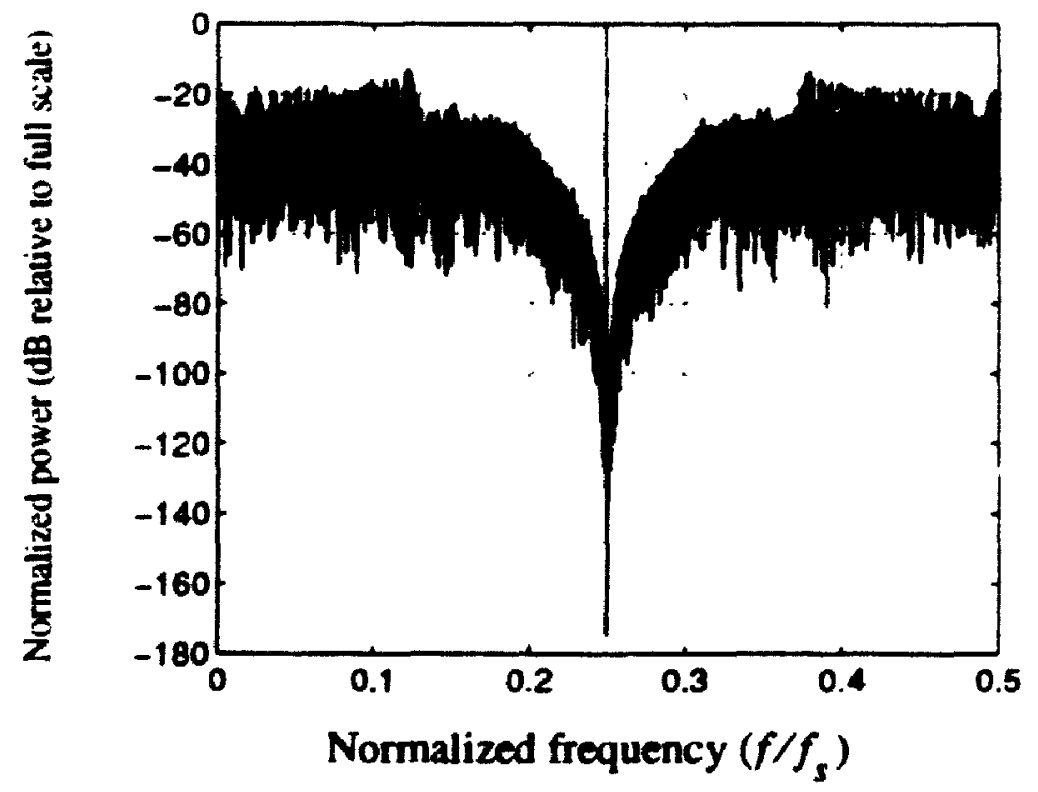

Figure 4.3: Simulated output spectrum of the fourth-order bandpass $\Sigma \Delta$ modulator in Figure 4.1 for a sinusoidal input.

The noise shaping is clearly seen at around a quarter of the sampling frequency. As we discussed in Chapter 2, this fourth-order modulator is guaranteed to be stable because of 
the itability of the second-order lowpass prototype.

The $f_{s} / 4$ i sonator can be implemented in several different ways using SC techniques. In [Singor94], resonators are implemented using Losiless Discrete Integrators (LDI) and Forward-Euler (FE) integrators. Another approach is to use two delay cells in a negatuve feedback loop [Longo93], as shown in Figure 4.4.

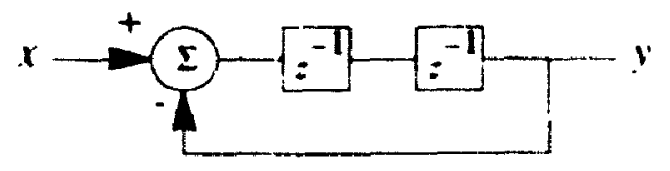

Figure 4.4: Resonator using delay cells

The latter design is chosen here for SC implementation because: (1) it operates at a higher speed [Singor94], (2) an SC delay circuit is immune to capacitor non-linearity [Bazarjani95c] which is useful when a SC circuit is implemented by weakly non-linear MOSFET capacitors and (3) a variation of this architecture can be implemented using a high-speed bipolar sample-and-hold circuit [Varelas96].

\subsection{Half Delay SC Circuits}

There are many SC amplifier designs that contain a half delay in their operation [Gregorian86]. Some involve techniques to reduce DC offset and $1 / f$ noise [Gregorian81], others utilize gain-enhancing methods to compensate for low opamp ICC gain [Haug84].

Here, the delay cell is intended to be used in a bandpass $\Sigma \Delta$ modulator, thus. offset and $1 / f$ noise do not affect the performance of the circuit. Moreover, sampled-data $\Sigma \Delta$ modulators typically require moderate opamp DC gain (opamp DC gain must be comparable to the oversampling ratio) and, as such, a gain-compensation scheme may not be needed. A simple SC amplifier is used for speed considerations.

The circuit of a fully differential SC amplifier is shown in Figure 4.5a. A two-phase nonoverlapping clock, as shown in Figure 4.5b, is required for the operation of this circuit. The output is delayed by a half-clock period and has a gain of $C_{S} / C_{H}$. Assuming infinite opamp DC gain and denoting the differential input and output by $v_{o d}$ and $v_{t d}$, where 


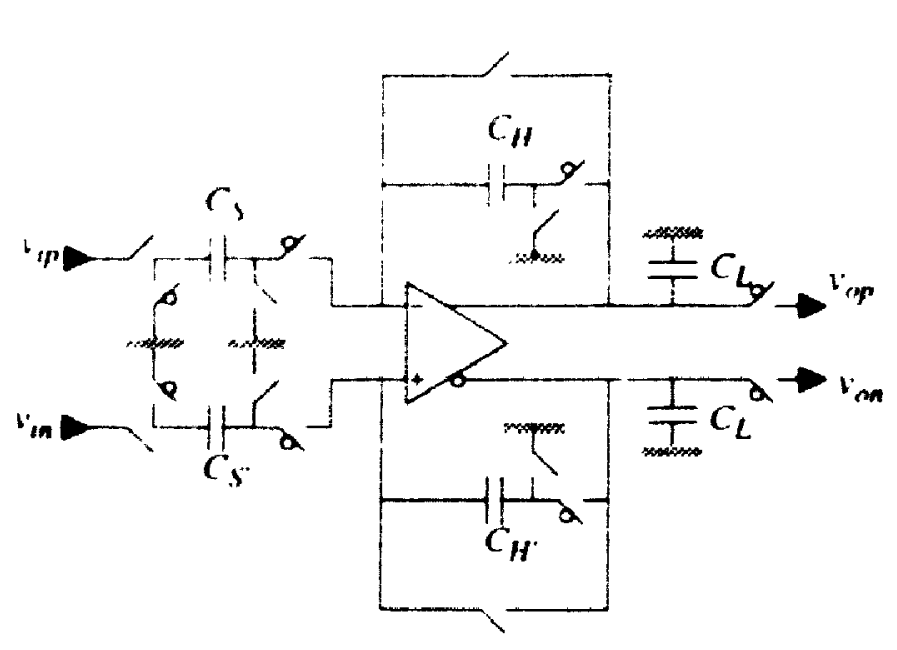

(a)

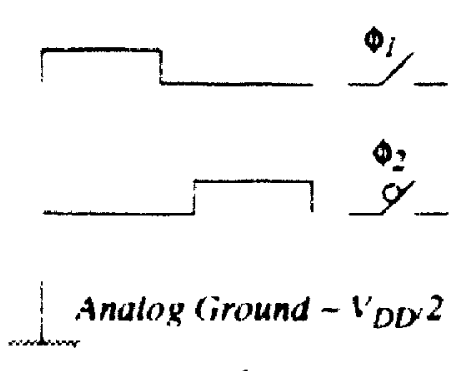

(b)

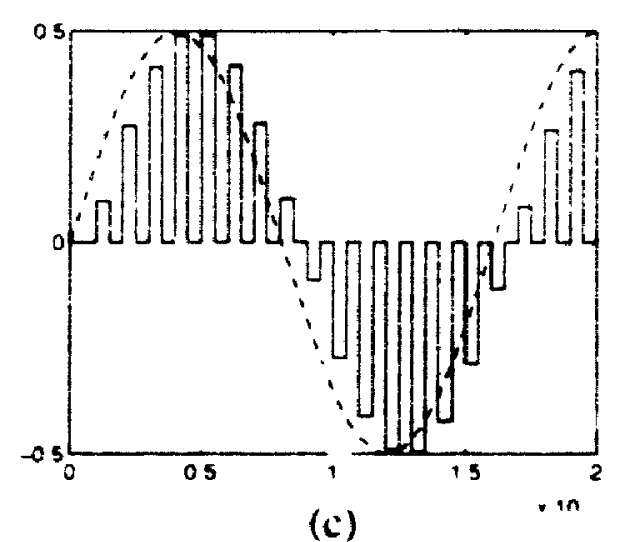

Figure 4.5: (a) A SC half delay gain stage. (b) 2-phase non-overlapping clock. (c) input (dashed line) and output (solid line) waveforms

$$
v_{o d}=v_{o p}-v_{o n} \text { and } \quad v_{i d}=v_{i p}-v_{i n} \text {. }
$$

the : -domain transfer function of this amplifier (output sampled during $\phi_{2}$ ) is

$$
\frac{V_{o d}(z)}{V_{i d}(z)}=H_{H D}(z)=\frac{C_{S}}{C_{H}} z^{-1 / 2}
$$

If the sampling capacitor, $C_{S}$, and the holding capacitor. $C_{H}$, are identical, the circuit is called a unity gain buffer or sample- and-hold circuit.

The ideal transfer function of the SC amplifier is affected by a number of non-ideal circuit behaviors. The impacts of finite opamp gain, incomplete settling, switch charge-injection, circuit noise, and capacitor mismatch on the performance of the half delay SC amplifier are analyzed in the following section.

\section{Finite Opamp Gain Errors}

Finite DC opamp gain ( $A$ ) and a non-zero opamp input capacitance $\left(C_{\text {in }}\right)$ introduce gain error in (4-5). as analyzed below. Figure 4.6 shows single-ended equivalent circuits of 
Figure $4.5 \mathrm{a}$ during $\phi_{1}$ and $\phi_{2}$ phases.

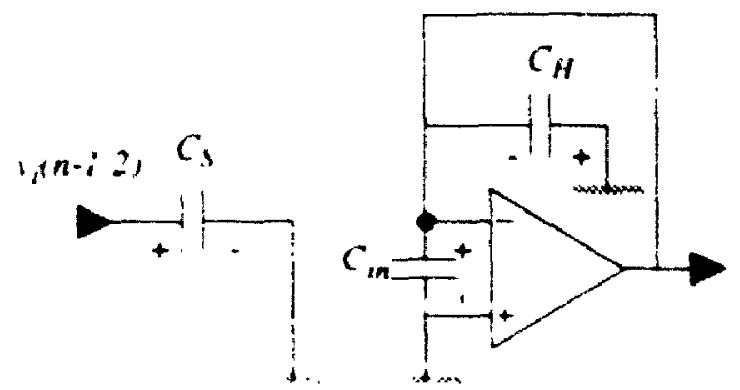

(a)

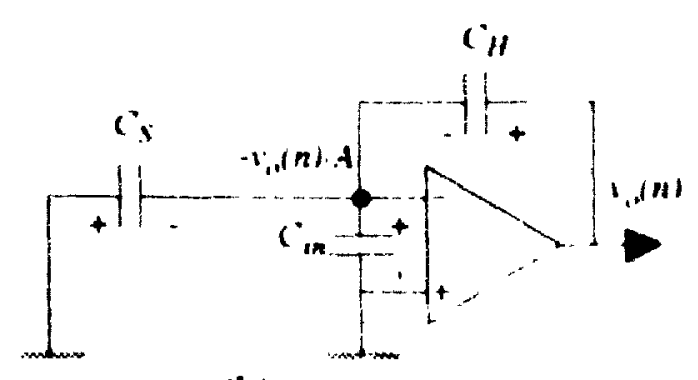

(b)

Figure 4.6: A single-ended equivalent circuit of Figure 4.5a dunng (a) $\phi_{1}$ and (b) $\phi_{2}$ phases

Charge conservation on capacitors $C_{s} \cdot C_{H}$, and $C_{\text {tn }}$ before and after $\phi_{2}$ yields the following difference equation:

$$
C_{S}\left[v_{i}(n-1 / 2)-\frac{v_{0}(n)}{A}\right]-C_{H}\left[v_{0}(n)+\frac{v_{0}(n)}{A}\right]=C_{i n} \frac{v_{0}(n)}{A}
$$

In the z-domain the actual transfer function of the fully differential SC amplitier becomes

$$
H_{H D}(z)=\frac{V_{o d}(z)}{V_{i d}(z)}=\frac{C_{S}}{C_{H}} \cdot \frac{1}{1+\frac{1}{A \beta}} \cdot z^{-1 / 2}
$$

where $\beta$ is the feedback factor and is given by

$$
\beta=\frac{C_{H}}{C_{H}+C_{S}+C_{\text {in }}}
$$

Here, $C_{\text {in }}$ represents the sum of all parasitic capacitances appearing at the input of the opamp. including opamp input capacitance. If $A \beta * 1$, the transfer function of (4-7) can be simplified to

$$
H_{H D}(z)=\frac{C_{S}}{C_{H}}\left(1-\frac{1}{A \beta}\right) \cdot z^{-1 / 2}
$$

An opamp with $50 \mathrm{~dB}$ DC gain $(A=300)$ causes $1 \%$ error in a sample-and-hold circuit 
if the input capacitance $C_{\text {in }}$ of opamp is comparable to $C_{S}$ and $C_{H}$.

\section{Settling Errors}

Another source of error in the half delay circuit is due to incomplete settling. During the hold phase $\left(\phi_{2}\right)$, the opamp is connected in a negative feedback configuration and is modelleci by Figure 4.7.

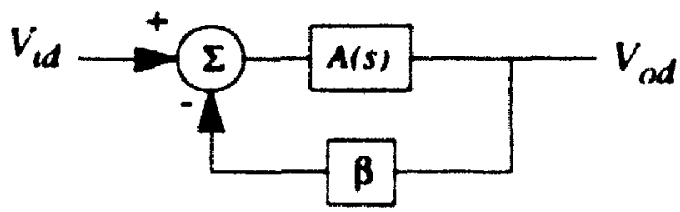

Figure 4.7: Opamp in the closed-loop configuration during $\phi_{2}$

If the opamp is a single-stage topology with a gain of $A=\omega_{u} / s$, the closed-loop transfer function wiil tre

$$
\frac{V_{o t}}{V_{i u^{\prime}}}(s)=\frac{A(s)}{1+\beta A(s)}=\frac{\omega_{u}}{s+\beta \omega_{u}} .
$$

Thus, the output of the SC half delay amplifier follows an exponential behavior as follows:

$$
v_{o d}=v_{F}\left(1-e^{-t / \tau}\right)
$$

Here. $v_{f}$ is the final output value and $\tau$ is the closed-loop time constant given by

$$
\tau=\frac{1}{\beta \omega_{u}}
$$

where $\omega_{\mu}$ is the open-loop unity gain frequency of the opamp. The hold time of the SC half delay circuit is about half a clock period. Therefore, the output cannot reach the exact tinal value and there will be a settling error. The required time constant to settle to $\alpha$ percent of the final value in a half clock period $\left(T_{s} / 2\right)$ is given by

$$
\tau=\frac{T_{s} / 2}{\ln (100 / \alpha)}
$$


For instance, settling to $0.1 \%$ of the final value requires the time constant to be $\tau \leq\left(T_{s} / 13.8\right)$

In the time constant equation (4-12), the unity gain frequency is determined by

$$
\omega_{u}=\frac{\boldsymbol{s}_{m}}{C_{T L}}
$$

where $q_{m}$ is the transconductance of the opamp and $C_{T L}$ is the total load capacitance appeanng at the opamp output during $\phi_{2}$, which in the SC amplitier of Figure 4.5 is

$$
C_{T L}=C_{L}+\frac{C_{H}\left(C_{S}+C_{i n}\right)}{C_{H}+\left(C_{S}+C_{i n}\right)} .
$$

$C_{L}$ is the opamp load capacitance plus all the parasitic capacitances at the output of the opamp.

\section{Switch Charge Injection Errors}

A third source of error is due to charge injection from MOSFET switches. Consider a simple sample-and-hold circuit comprised of an nMOSFET switch and a holding capacitor $C_{H}$ as shown in Figure 4.8.

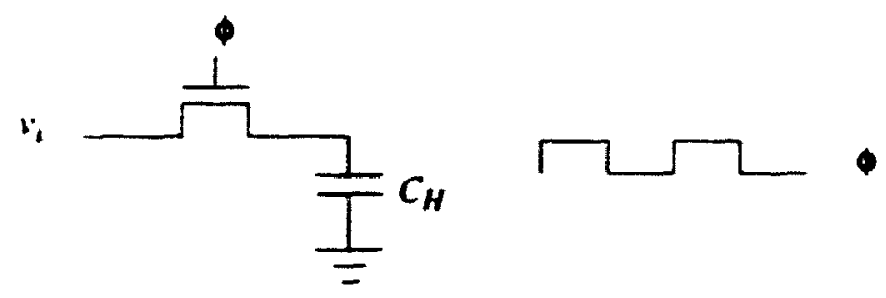

Figure 4.8: A simple SC sample-and-hold circuit

At the end of the sampling phase, $\phi=V_{D D}$, the holding capacitor reaches a voltage that is equal to the input voltage $v_{i}$ (assuming the setting error is negligible). During the hold phase, $\phi=0$, the clock voltage is dropped from $V_{D D}$ to zero and ideally the voltage on the holding capacitor is held constant and equal to the value of the input voltage at the end of the sampling phase. However, the sampled voltage is disturbed due to channel charge injection and clock feedthrough when the nMOSFET switch is turned off. The inversion channel charge in a turned-on nMOSFET is given by 


$$
Q_{1 h}=C_{o x}(W L)\left(v_{g s}-V_{t}\right)
$$

When the transistor is turned off, this charge is released to drain, source and substrate. If the fall time of the clock is longer than the channel transit time i $\left.\tau_{1}=L^{2} / \mu_{n}\left(v_{(j)}-V_{1}\right)\right)$. the charge absorbed by the substrate is negligible. The charge partition between source and drain is a funcion of clock fall time and drain/ source capacitance. For a sharp clock edge, channel charge is divided equally between source and drain sides [Wegmann87].

The error introduced in the sample-and-hold circuit is

$$
\Delta V=-\alpha \cdot \frac{C_{o x}(W L)\left(V_{D D}-v_{i}-V_{i}\right)}{C_{H}}
$$

where $\alpha$ is the percent of channel charge injected to the holding capacitor. This channel charge causes offset. gain error and distortion in the sample-and-hold circuit.

A method for eliminating the signal dependent charge injection is bottom plate sampling [Gregorian86], as illustrated in Figure 4.9. The channel charge associated with the

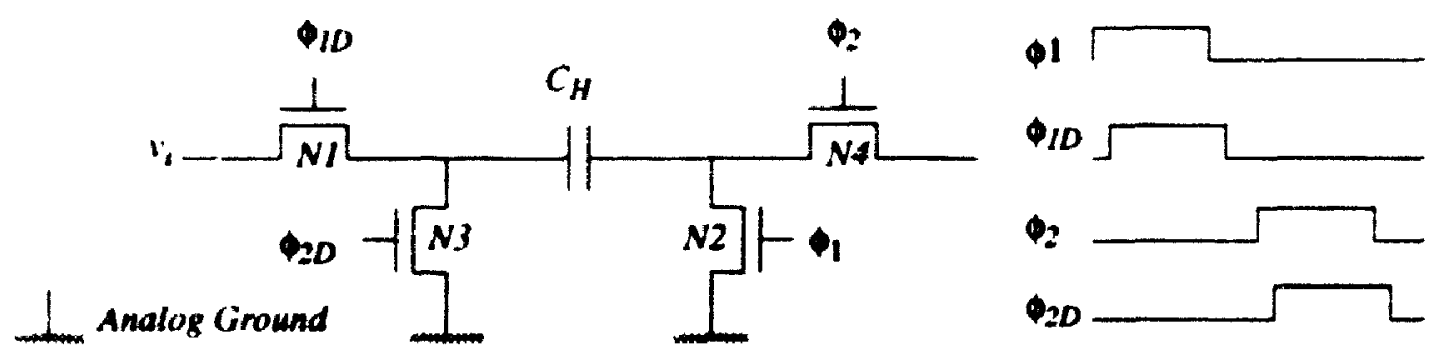

Figure 4.9: Bottom-plate sampling technique to eliminate signal dependent charge injection

nMOSFET switch $N 2$ is independent of the signal because it is connected to analog ground. Switch $N 2$ is opened first, thus the charge injected to the sampling capacitor is signal independent. Charge injected by the switch $N 1$ to the top plate causes no error because it is shorted to ground in the next phase.

Another charge injection error is due to clock coupling through the gate to drain overlap capacitance. In a fully differential architecture. Figure 4.10 , both clock feedthrough and 
tixed channel charge is cancelled.

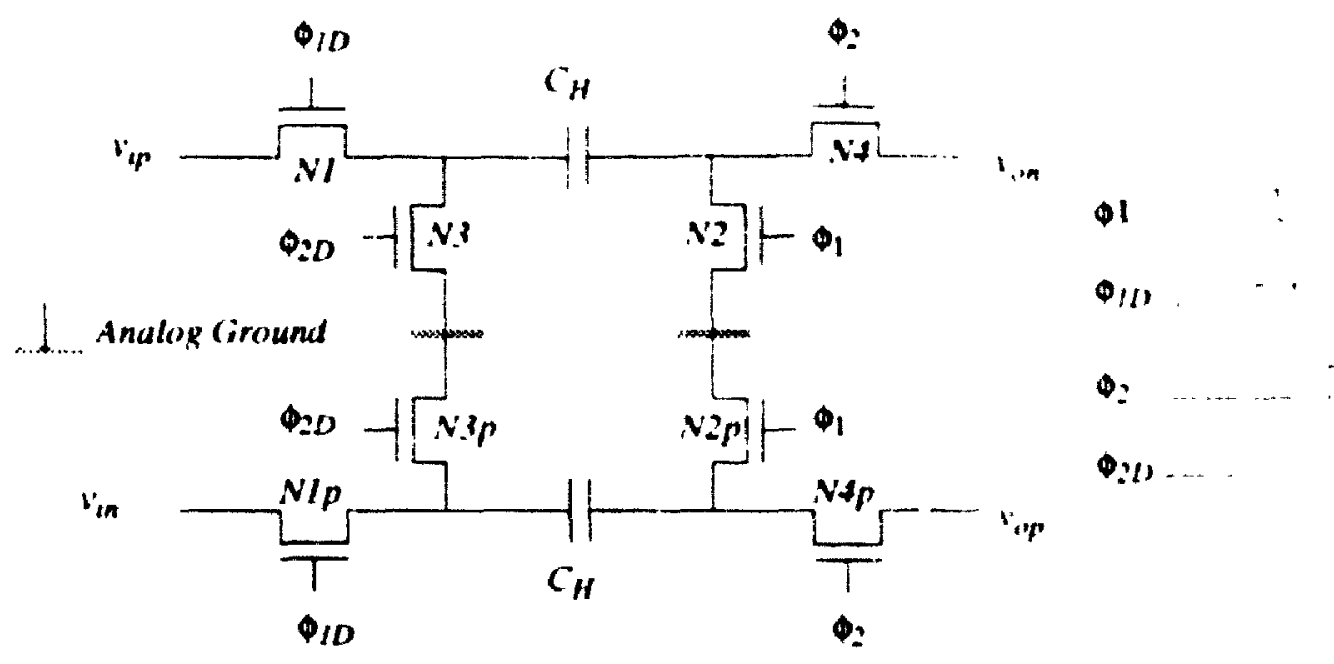

Figure 4.10: Fully differential bottom-plate sampling to cancel tixed charge injection and clock feedihrough

\section{Noise Errors}

In SC circuits, thermal and ficker $(1 / f)$ noise generated by transistors used in opamps and analog switches determine the minimum detectable signal amplitude. The power of ficker noise at high frequencies is very low and therefore its effect on the performame it high frequency SC circuits can be neglected. Thermal noise due to on-resistance of the analog switches and opamp wide-band noise is analyzed for a single-ended SC' amplitier. During the sampling phase. Figure 4.11 a, there will be a mean syuared noise charge

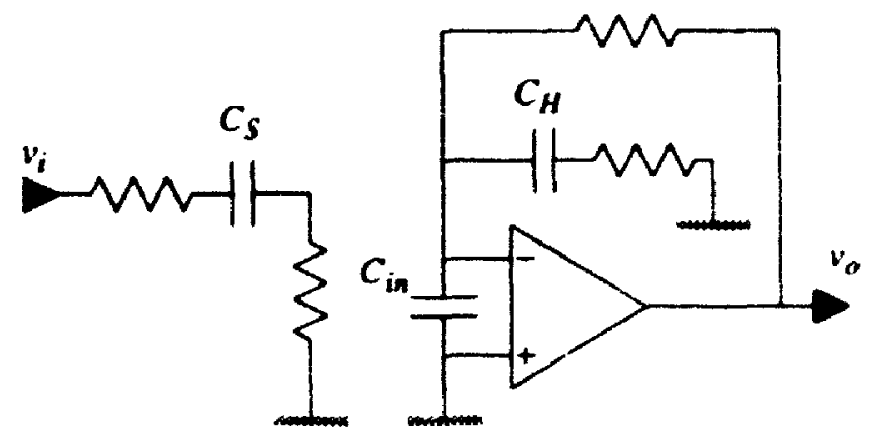

(a)

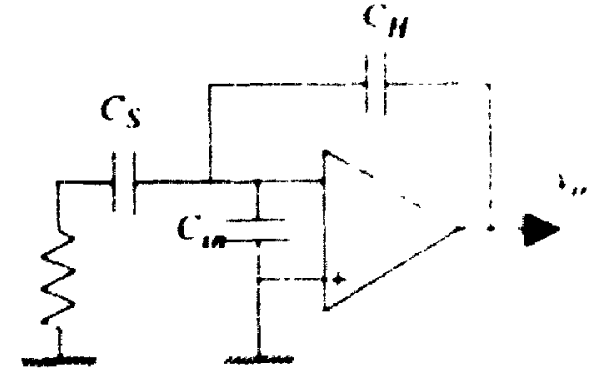

(b)

Figure 4.11: Single-ended equivalent circuits of Figure 4.5a during (a) sampling phase, and (b) holding phase

( $k T C$ ) on both sampling capacitor $C_{5}$ and holding capacitor $C_{H}$. In the hold phase $\varphi_{2}$. 
Figure $4.11 \mathrm{~b}$, there will be a new $k T C_{S}$ noise on sampling capacitor. The thermal noise on holding capacitor during $\Phi_{2}$ is at low frequencies and will not affect the circuit. The net noise on the sampling capacitor (i.e. $2 k T C_{S}$ ) is transferred to the holding capacitor and the total charge noise power at the ouput will be

$$
q_{n}^{2}=k T\left(C_{H}+2 C_{S}\right)
$$

The mean squared $k T / C$ noise voltage at the output will be

$$
\nabla_{n(\text { out })}^{2}=\frac{\bar{q}_{n}^{2}}{C_{H}^{2}}=\frac{k T}{C_{H}}(1+2 G)
$$

where $G=C_{S} / C_{H}$ is the gain of the SC amplifier. In a fully differential SC architecture, there are twice as many capacitors as in a single-ended SC circuit, thus the noise power is doubled. Moreover, for a constant power dissipation and speed, capacitor sizes in a fully differential circuit must be half the size (thus having twice the noise power) of a singleended circuit. Therefore, the thermal $k T / C$ noise of a fully differential circuit will be four times higher than that of a single-ended circuit. However, the voltage swing of a fully differential circuit is twice as high ( 4 times in power) as that of a single-ended SC circuit. Thus, for a given speed and power dissipation the SNR will be equal for both a fully differential and a single-ended SC circuit.

The thermal noise associated with an opamp has a power spectral density of at least

$$
S_{\text {oTA }}(f)=4 k T\left(\frac{2}{3} \cdot \frac{1}{g_{m}}\right)
$$

Assuming a single-pole opamp and using the equivalent noise bandwidth $\left(\omega_{3 d B} / 4\right)$ introduced in [Gray93], the total opamp thermal noise power will be

$$
\nabla_{n T(o t a)}^{2}=\frac{2}{3} \cdot \frac{k T}{C_{T L}} \cdot \beta \text {, }
$$

where $\beta$ and $C_{T L}$ are given by (4-8) and (4-15) respectively. The input referred overall voltage noise power of the SC amplifier can be expressed as 


$$
\nabla_{n}^{2}=k T\left(\frac{1+2 G}{C_{H}}+\frac{2}{3} \cdot \frac{\beta}{C_{T L}}\right)\left(\frac{C_{H}}{C_{S}}\right)^{2}
$$

In a sample-and-hold circuit where $C_{S}=C_{H}=C_{\text {in }}=C_{L}=C_{u}$. the RMS value of the input referred thermal noise voltage is

$$
r_{n . i}=\sqrt{\frac{47}{15} \cdot \frac{k T}{C_{u}}}
$$

If the above sample-and-hold is changed to a SC amplifier with a gain of 0.5 (by changing $C_{H}=2 C_{u}$ ) the input referred thermal noise will be

$$
\nabla_{n, i}=2 \sqrt{\frac{7}{6} \cdot \frac{k T}{C_{u}}}
$$

\section{Capacitor Mismatch Error}

Another source of gain error is due to capacitor mismatch. Device matching is technology and layout dependent. Dielectric thickness variation and edge errors across the die contribute to inaccuracy in capacitance matching. In general, matching is improved as the size of the devices to be matched is increased. Proximity and having similar geometry also help to achieve better matching. Typically, poly-to-poly capacitor matching in the order of $1 \%$ to $0.1 \%$ is readily achievable in many analog CMOS processes.

An efficient architecture for unity gain sample-and-hold exists which is immune to capacitor mismatch and requires one capacitor to perform both sample and hold operations, as shown in Figure 4.12.

In this circuit, during $\phi_{1}$ input voltage is stored on sampling capacitor $C_{S}$ and during $\phi_{2}$ capacitor $C_{S}$ is switched to the output and plays the role of holding capacitor. Thus, gain error due to mismatch between sampling capacitor and holding capacitor is irrelevant. Furthermore, the one-capacitor sample-and-hold structure has other advantages over the two-capacitor version. In the one-capacitor sample-and-hold circuit, finite opamp gain $A$ still causes gain error and the transfer function is 


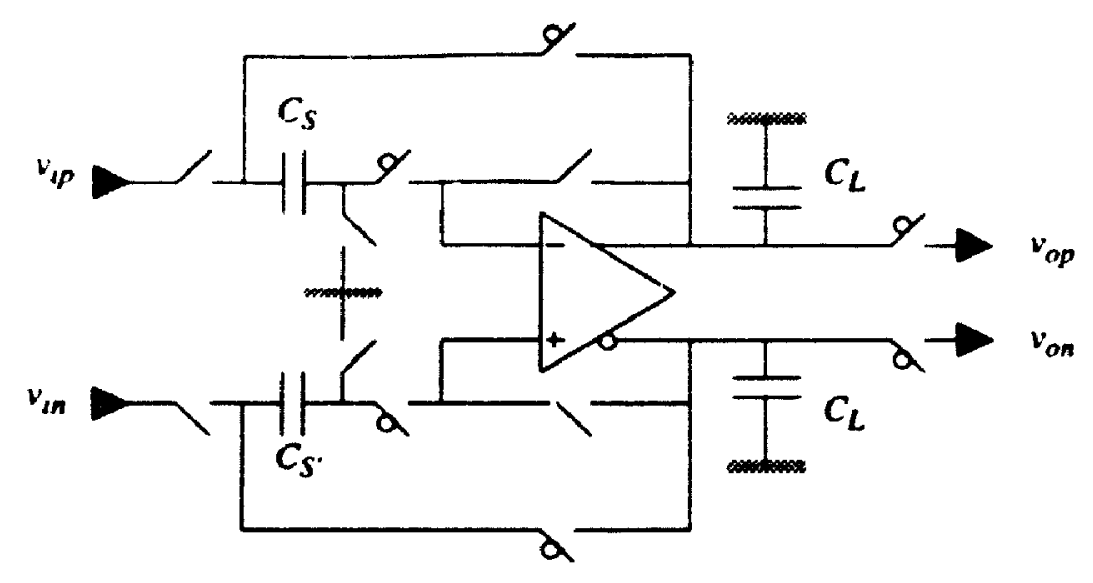

Figure 4.12: One capacitor sample-and-hold circuit

$$
H(z)=\frac{V_{\text {od }}}{V_{\text {id }}}(z)=\frac{1}{1+\frac{1}{A \beta}} \cdot z^{-1 / 2},
$$

where the feedback factor $\beta$ is given by

$$
\beta=\frac{C_{s}}{C_{S}+C_{i n}} .
$$

Compared to the two-capacitor sample-and-hold circuit, the one-capacitor sample-andhold structure has a higher $\beta$ and therefore a lower gain error.

The total load capacitance in this sample-and-hold circuit is

$$
c_{T L}=C_{L}+\frac{C_{S} C_{i n}}{C_{S}+C_{i n}} .
$$

The value of $C_{r L}$ is less in a single capacitor sample-and-hold circuit (4-27) than in a two-capacitor sample-and-hold circuit (4-15). From equation (4-14), a lower equivalent output capacitance results in a higher unity gain frequency $\omega_{u}$. Therefore, the closed loop time constant (4-12) of the one-capacitor sample-and-hold is smaller than the closed-loop time constant of a two-capacitor sample-and-hold circuit due to both higher $\omega_{u}$ and higher $\beta$.

In the one-capacitor sample-and-hold circuit, the sampling capacitor is the only source of thermal $k T / C$ noise. Therefore, the output $k T / C$ noise power (which is the same as 
input referred noise power) in the one-capacitor sample-and-hold circuit is $k T / C_{S}$ and is $5 \mathrm{~dB}$ lower than the two-capacitor sample-and-hold circuit. Again. in a fully differential structure, the noise power is doubled and is equal to $2 k T / C_{s}$.

Example 4.1: In sample-and-hold circuits of Figure 4.5 and Figure 4.12, if opamp input capacitance $C_{\text {in }}$ and output load $C_{L}$ are equal to the sampling capacitor $C_{S}$ (and holding capacitor $C_{H}$ ), the one-capacitor sample-and-hold SC circuit is about 1.6 times faster than the two-capacitor sample-and-hold SC circuit.

\subsection{Full Delay SC Circuits}

The SC amplifier of Figure 4.5 performs a half delay operation. A simple full delay SC circuit is obtained by cascading two of these half delay circuits with alternate $\phi_{1}$ and $\phi_{2}$ clock phases as shown in Figure 4.13. This two-stage SC amplifier circuit delays the inpui

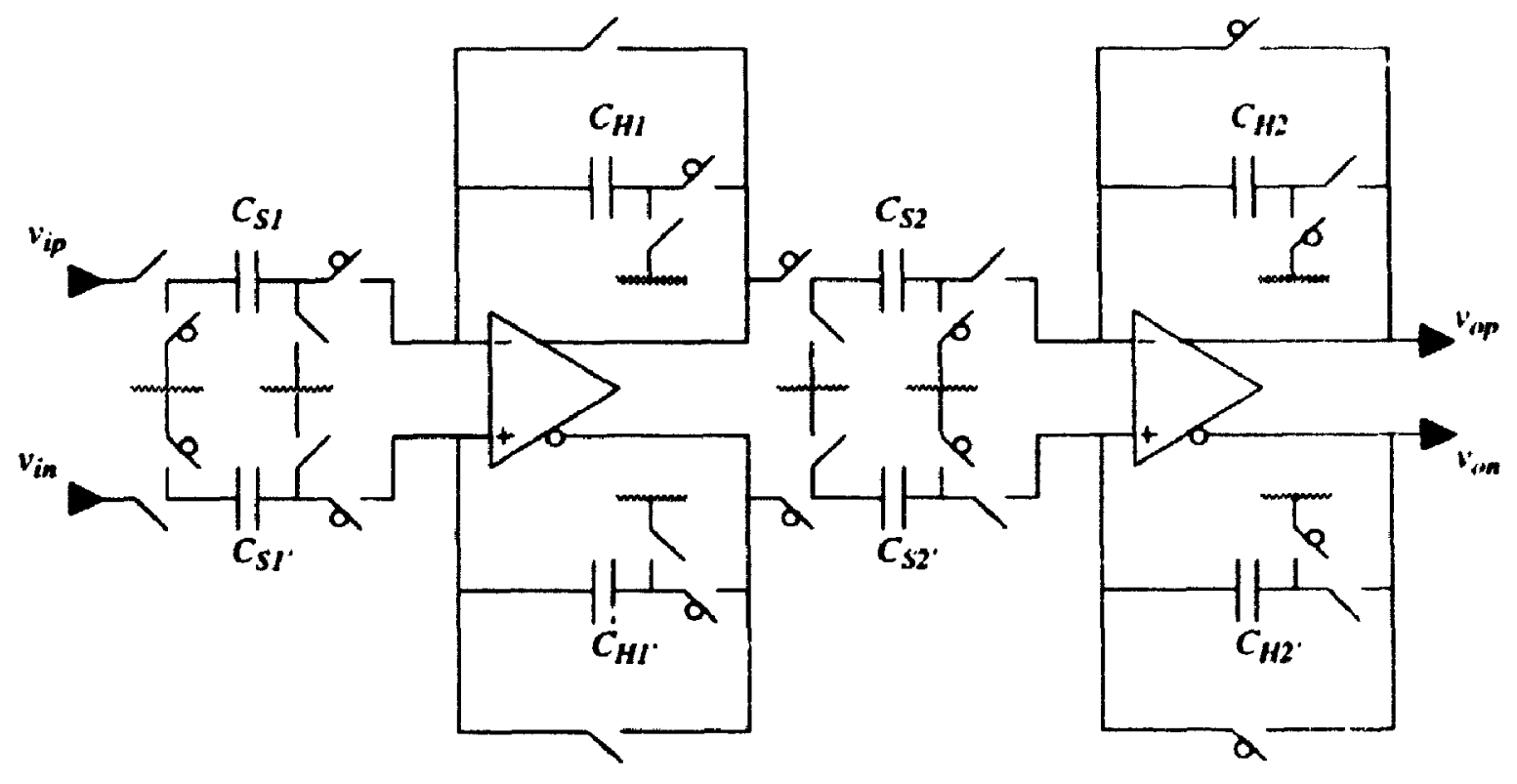

Figure 4.13: A simple cascade SC delay circuit

signal for a full clock period and scales it. The $z$-domain transfer function of this circuit is

$$
\frac{V_{\text {od }}}{V_{i d}}(z)=\frac{C_{S 1}}{C_{H 1}} \cdot \frac{C_{S 2}}{C_{H 2}} \cdot z^{-1} .
$$

Both opamps in this configuration are idle for a half clock period. A more efficient full delay gain stage circuit is achieved by merging the two half delay circuits into a cell using 
one opamp [Longo93], as shown in Figure 4.14. The $z$-domain transfer function of this

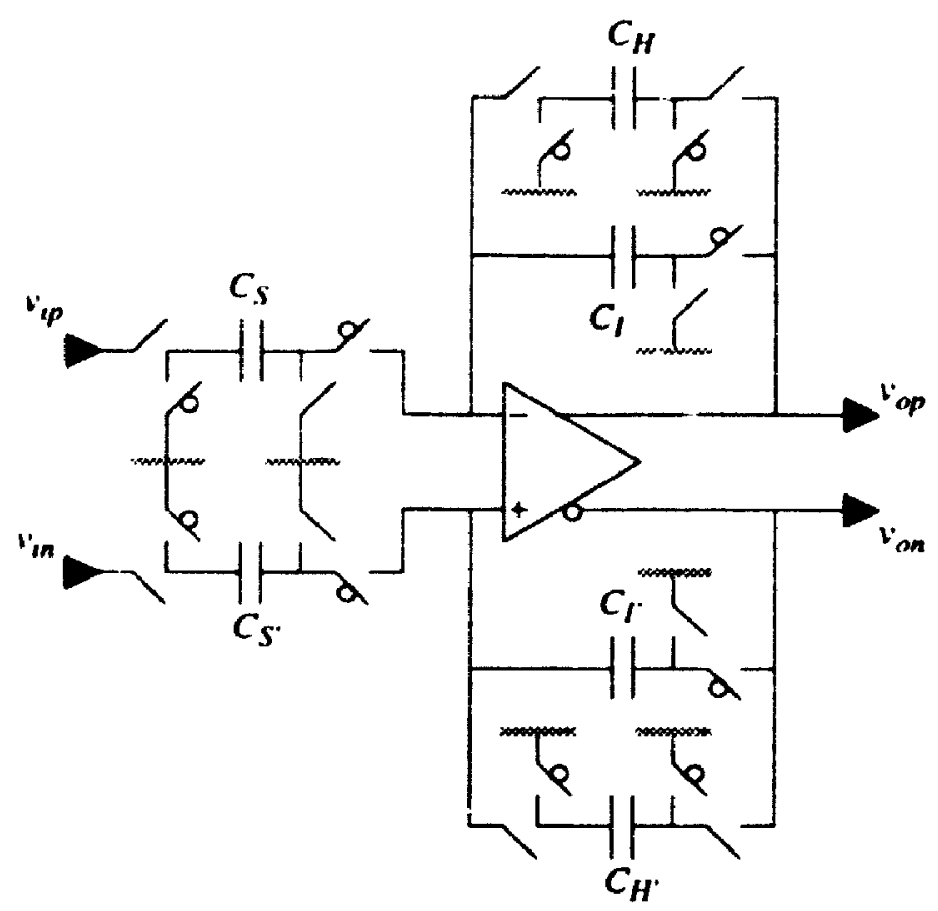

Figure 4.14: A SC delay gain stage

circuit is

$$
H_{D}(z)=\frac{V_{o d}}{V_{i d}}(z)=\frac{C_{S}}{C_{H}} \cdot z^{-1}
$$

Finite opamp gain causes gain and phase error in the full delay circuit of Figure 4-14, as analyzed below. Single-ended equivalent circuits for the SC delay circuit of Figure 4.14 during $\phi_{1}$ and $\phi_{2}$ are depicted in Figure 4.15. An opamp gain of $A$ causes the virtual

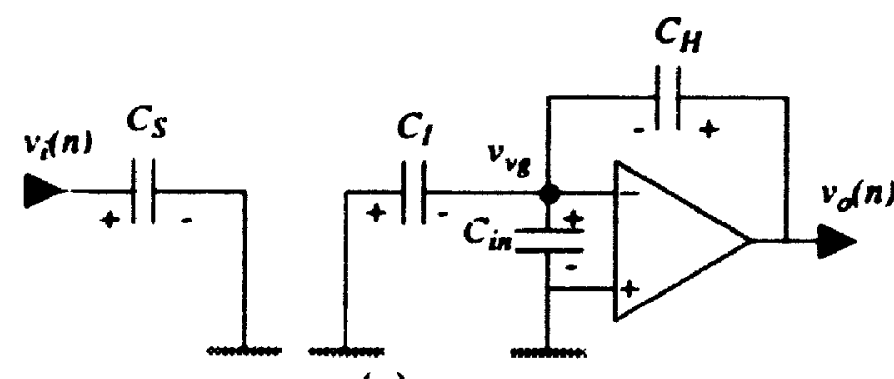

(a)

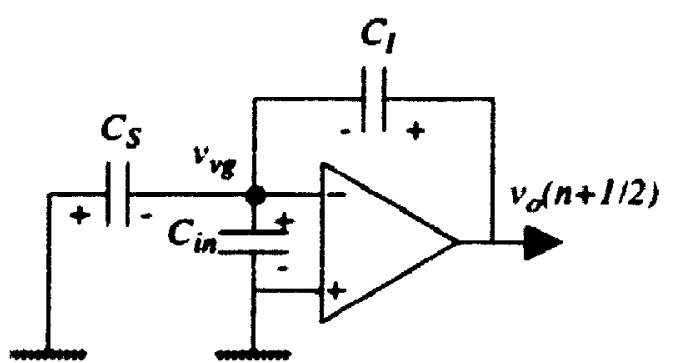

(b)

Figure 4.15: A single-ended equivalent circuit of Figure 4.14 during (a) $\phi_{1}$ and (b) $\phi_{2}$ phases 
ground node of the opamp $v_{v g}$ to be at $-v_{0} / A$ with respect to analog ground. The charge conservation equation before and after $\phi_{2}$ yields the following difference equation:

$$
\begin{aligned}
& C_{S}\left[v_{1}(n)-\frac{v_{0}(n+1-2)}{A}\right]+C_{1}\left[\frac{v_{0}(n)}{A}-\left(v_{0}(n+1,2)+\frac{v_{1}(n+1 \cdot 2)}{A}\right)\right] \\
& =C_{i n}\left[-\frac{-1(n)}{A}+\frac{i_{1}(n+12)}{A}\right]
\end{aligned}
$$

In the $z$-domain. the transfer function of the half delay circuit (during $\phi_{2}$ in Figure $4-14$ ) becomes

$$
H_{H D}(z)=\frac{C_{S}}{C_{I}}{ }^{-1 / 2}\left[\frac{g_{0}}{1-p_{0} z^{-1 / 2}}\right]
$$

where $g_{0}$ and $p_{0}$ are given by

$$
g_{0}=\frac{1}{1+1 / A \beta} \quad p_{0}=\frac{g_{0}}{A}\left(1+\frac{C_{\text {in }}}{C_{1}}\right),
$$

where $\beta=C_{I} /\left(C_{1}+C_{S}+C_{\text {in }}\right)$. If $1 / A \beta$ \& 1 , the above equations can be simplitied to

$$
g_{0}=1-1 / A \beta \quad p_{0}=\frac{1}{A}\left(1+C_{\text {in }} / C_{1}\right)
$$

The term in the square bracket of equation (4-31) is the error term $E(z)$. The frequency response of the error term is derived by substituting $z=e^{j \omega T}$ in $E(z)$, and is given by

$$
E\left(e^{j \omega T}\right)=\frac{g_{0}}{1-p_{0} \cos (\omega T / 2)+j p_{0} \sin (\omega T / 2)}
$$

The magnitude $|E(\omega)|$ and the phase $\angle E(\omega)$ of the above equation are

$$
\begin{aligned}
|E(\omega)|^{2}= & \frac{g_{0}^{2}}{\left[1-p_{0} \cos (\omega T / 2)\right]^{2}+\left[p_{0} \sin (\omega T / 2)\right]^{2}} \\
& \angle E(\omega)=-\tanh \frac{p_{0} \sin (\omega T / 2)}{1-p_{0} \cos (\omega T / 2)} .
\end{aligned}
$$

For $A \geqslant 1$, we have $p_{0} \ll 1$ and the magnitude and phase of the error term can be approximated as 


$$
\begin{gathered}
|E(\omega)|=m_{H D}=1-\frac{1}{A \beta}+\frac{\cos (\omega T / 2)}{A}\left(1+\frac{C_{\text {in }}}{C_{H}}\right) \\
\angle E(\omega)=\theta_{H D}=-\frac{\sin (\omega T / 2)}{A}\left(1+\frac{C_{\text {in }}}{C_{H}}\right) .
\end{gathered}
$$

Therefore, the transfer function of the half delay SC circuit with finite opamp gain will be

$$
H_{H D}(z)=\frac{C_{S}}{C_{I}} z^{-1 / 2}\left[m_{H D} \cdot e^{j \theta_{H D}}\right]
$$

The full delay SC circuit of Figure 4.14 will experience errors during $\phi_{1}$ and $\phi_{2}$ and the actual transfer function of the full delay circuit will be

$$
H_{F D}(z)=\frac{C_{S}}{C_{H}} z^{-1}\left[\frac{\left(g_{0}\right)_{\phi_{1}}}{1-\left(p_{0}\right)_{\phi_{1}} z^{-1 / 2}} \cdot \frac{\left(g_{0}\right)_{\phi_{2}}}{1-\left(p_{0}\right)_{\phi_{2}} z^{-1 / 2}}\right]
$$

Substituting $z=e^{j \omega T}$ in the above equation and assuming $\left(p_{0}\right)_{\phi_{1}}$ and $\left(p_{0}\right)_{\phi_{2}} * 1$, the magnitude and the phase of the error term in a full delay circuit due to finite opamp gain are obtained to be

$$
\begin{gathered}
m_{F D}=1-\frac{1}{A}\left[\frac{1}{\beta_{\phi_{1}}}+\frac{1}{\beta_{\phi_{2}}}\right]+\frac{\cos (\omega T / 2)}{A}\left[\left(1+C_{i n} / C_{l}\right)+\left(1+C_{i n} / C_{H}\right)\right] \\
\theta_{F D}=-\frac{\sin \omega T / 2}{A}\left[\left(1+C_{i n} / C_{I}\right)+\left(1+C_{i n} / C_{H}\right)\right],
\end{gathered}
$$

where $\beta_{\phi_{1}}$ and $\beta_{\phi_{2}}$ are the feedback factor during $\phi_{1}$ and $\phi_{2}$ respectively.

The actual transfer function of a full delay circuit is

$$
H_{F D}(z)=\frac{C_{S}}{C_{H}} z^{-1}\left[m_{F D} \cdot e^{j \theta_{F D}}\right]
$$

Example 4.2: In the SC full delay circuit of Figure 4.14, opamp DC gain is 100 , signal is a sinusoid with frequency of $f_{S} / 4$ and $C_{S}=C_{I}=C_{H}=C_{i n}$. The gain and phase of the error term, calculated from (4-41) and (4-42) respectively, are 


$$
m_{F D}=0.968 \quad \theta_{F D}=-10.028
$$

Therefore, an opamp gain of $40 \mathrm{~dB}$ will cause $3.2 \%$ gain error in the full delay SC sample-and-hold circuit. The phase error is $2.8 \%$ (in the sense that $\left|e^{-j(1.112 x}-e^{\prime(1)}\right|$ is $2.5 \%$ of $e^{j 0}$ ).

\section{A Double-Sampled SC Delay Cell}

The opamp in the one-capacitor sample-and-hold circuit of Figure 4.12 is idle during the sampling phase $\phi_{1}$. By duplicating the sampling circuitry and using an alternate clock phase for it, a two-path SC sample-and-hold is obtained, as shown in Figure 4.16.

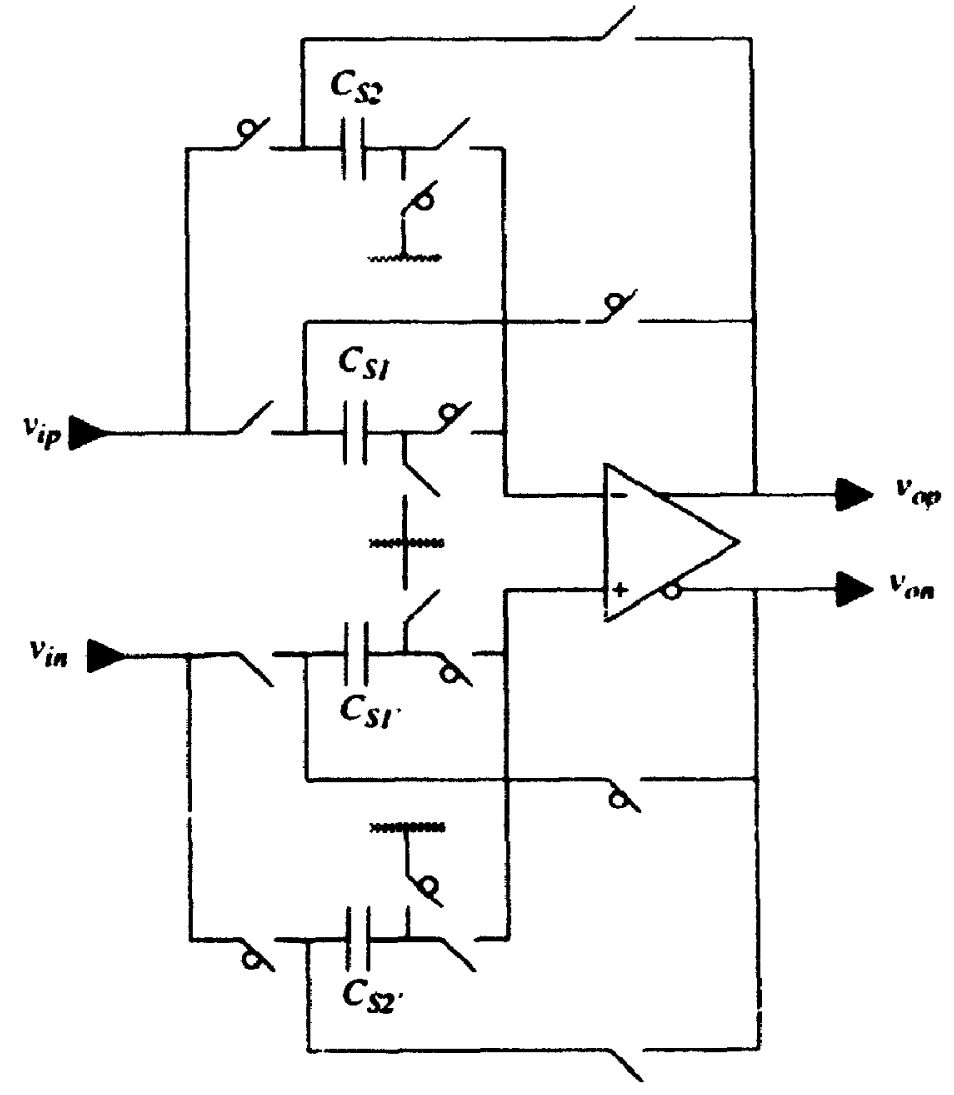

Figure 4.16: A double-sampled SC delay circuit

In this circuit, the input signal is sampled every half clock period $\left(T_{s} / 2\right)$ and appears at the output with a half-clock period delay. Thus, the transfer function of this cell is

$$
\frac{V_{\text {od }}}{V_{i d}}(z)=\hat{z}^{-1}
$$




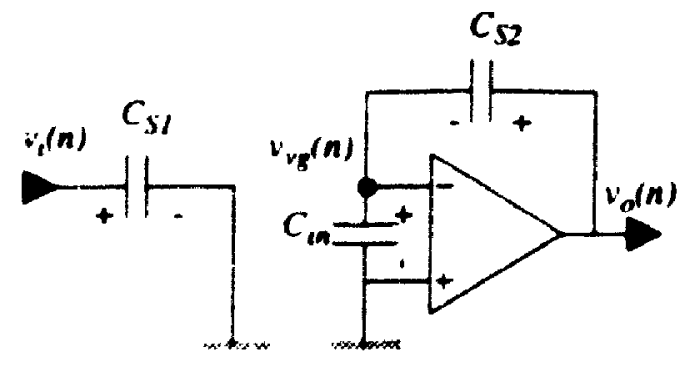

(a)

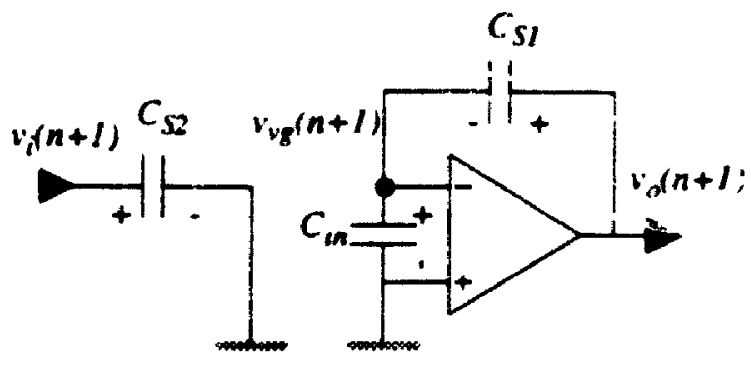

(b)

Figure 4.17: A single-ended equivalent circuit of Figure 4.16 during (a) $\phi_{1}$ and (b) $\phi_{2}$ phases

where $\hat{z}=e^{j \omega\left(T_{1} / 2\right)}$.

Therefore, the effective sampling frequency in this two-path sample-and-hold circuit is twice the clock frequency. This structure is also called a double-sampled SC circuit [Choi80] [Hurst90]. The factor-of-two improvement in the speed of the double-sampled SC delay cell is achieved without increasing the clock rate or requiring a faster opamp settling time. In return, mismatch and uneven clock phases create image errors as we will discuss later.

Finite opamp gain and opamp input capacitance cause both gain and phase error in the transfer function of the double-sampled SC delay circuit. Figure 4.17 shows a singleended equivalent circuit of the double-sampled delay circuit during $\phi_{1}$ and $\phi_{2}$. Charge conservation on capacitors $C_{S 1}$ and $C_{i n}$ before $\phi_{2}$ (the non-overlapping phase) and after $\Phi_{2}$ yields the following difference equation:

$$
C_{S 1}\left[v_{i}(n)-\left(v_{o}(n+1)+\frac{v_{o}(n+1)}{A}\right)\right]=C_{i n}\left[\frac{-v_{o}(n)}{A}+\frac{v_{0}(n+1)}{A}\right] .
$$

Here the time indices at the end of $\phi_{1}$ and $\phi_{2}$ are denoted by $n$ and $n+1$ respectively due to the double sampling property of the circuit. If capacitors $C_{S 1}=C_{S 2}=C_{S}$, a similar difference equation would be obtained for the $\phi_{1}$ phase and the overall transfer function in $z$-domain will be

$$
\frac{V_{o d}(z)}{V_{i d}(z)}=H_{d D}(z)=\left[\frac{g_{0}}{1-p_{0} z^{-1}}\right] z^{-1}
$$


where $g_{0}$ and $p_{0}$ are given by

$$
\begin{gathered}
g_{0}=\frac{1}{1+\frac{1}{A}+\frac{C_{\text {in }}}{C_{S}} \cdot \frac{1}{A}} \\
p_{0}=\frac{C_{\text {in }}}{C_{S}} \cdot \frac{g_{0}}{A} .
\end{gathered}
$$

Thus, tinite opamp gain and non-zero input capacitance modify the transfer function of the double-sampled delay cell by a damped integrator term from its ideal response. This change of transfer function causes both gain and phase error in the response of the delay circuit. The actual trarsfer function becomes

$$
H_{d D}(z)=\left[m_{d D} \cdot e^{j \theta}\right] \cdot z^{-1}
$$

where $m_{d D}$ and $\theta_{d D}$ are magnitude and phase of the error term in the double-sampled delay circuit. Assuming $A * 1$, the magnitude and phase error are given by

$$
\begin{gathered}
m_{d D}=1-\frac{1}{A \beta}+\frac{\cos \omega T}{A}\left(\frac{C_{\text {in }}}{C_{S}}\right) \\
\theta_{d D}=-\frac{\sin \omega T}{A}\left(\frac{C_{\text {in }}}{C_{S}}\right) .
\end{gathered}
$$

Example 4.3: In Figure 4.16, if the input capacitance of the opamp is equal to the sampling capacitor and the opamp has $40 \mathrm{~dB}$ DC gain, both gain error and phase error are $1 \%$ for a signal at one half the clock frequency.

A major limitation of double-sampled SC circuits is due to mismatch in the two paths [Gregorian86] that causes in-band image of the signal as described later. However. the double-sampled circuit of Figure 4.16 uses the one-capacitor sample-and-hold architecturi and its gain is not affected (to a first order) by capacitor mismatch.

Double-sampled SC circuits are a subset of a class of circuits called $\mathbf{N}$-path filters with $\mathbf{N}$ being equal to two. A diagram of a two-path circuit and its corresponding clock phases is shown in Figure 4.18. 
The non-overlapping clock has a frequency of $f_{c l o c k}$ and the effective sampling frequency $\left(f_{s}\right)$ of a double-sampled SC circuit is $f_{s}=2 f_{\text {clock }}$. The sequence of the signals during $\phi_{1}$ (odd samples) is denoted by an " $o$ " superscript and the sequence of the signals during $\phi_{2}$ (even samples) is denoted by an " $e$ " superscript. The odd and even sequences have a sampling frequency of $f_{\text {clock }}=f_{s} / 2$. The input sequence $v_{\text {in }}$ is a time-interleaved vector sum of odd $\left(v_{i n}^{\prime}\right)$ and even $\left(v_{\text {in }}^{e}\right)$ sequences and in the $z$-domain we have

$$
V_{\text {in }}(z)=v_{\text {in }}^{o}(z)+V_{\text {in }}^{e}(z)
$$

Similarly, the output sequence is expressed as

$$
V_{\text {out }}(z)=V_{\text {out }}^{o}(z)+V_{\text {out }}^{e}(z)
$$

where odd and even sequences are related by

$$
v_{\text {out }}^{o}(z)=H(z) V_{\text {in }}^{o} \quad V_{\text {out }}^{e}(z)=H(z) V_{\text {in }}^{e} \text {. }
$$

If the two paths are not symmetric and. for instance, there is a gain mismatch of $\delta$ between them. the input-output relation is

$$
V_{\text {out }}(z)=(1+\delta) H(z) v_{\text {in }}^{o}+H(z) v_{\text {in }}^{e}
$$

This equation can be expressed as

$$
V_{\text {out }}(z)=H(z)\left[V_{\text {in }}(z)+\delta V_{\text {in }}^{o}(z)\right]
$$

Therefore, a mismatch between the two channels is equivalent to having an attenuated image of the signal being applied at the input along with the real input. Figure 4.19 shows

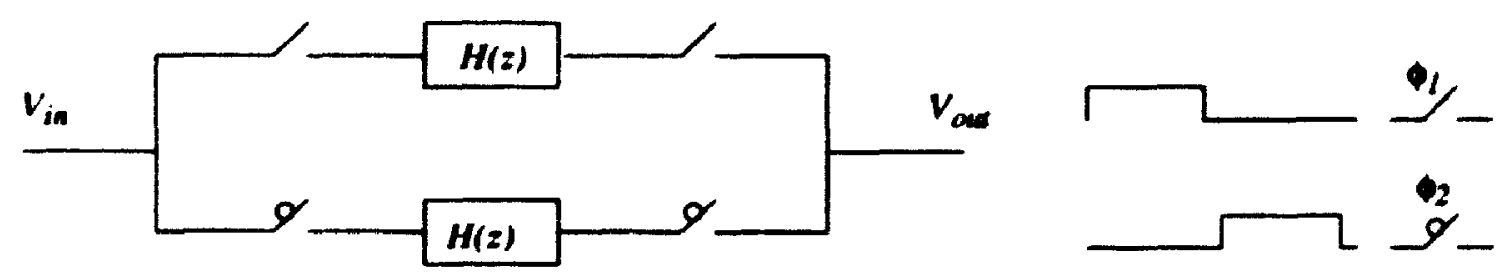

Figure 4.18: Two-path SC circuit and clock phases 
the periodic spectrum of the input signals $V_{\text {in }}$ and $\delta v_{\text {in }}^{o \prime}$.

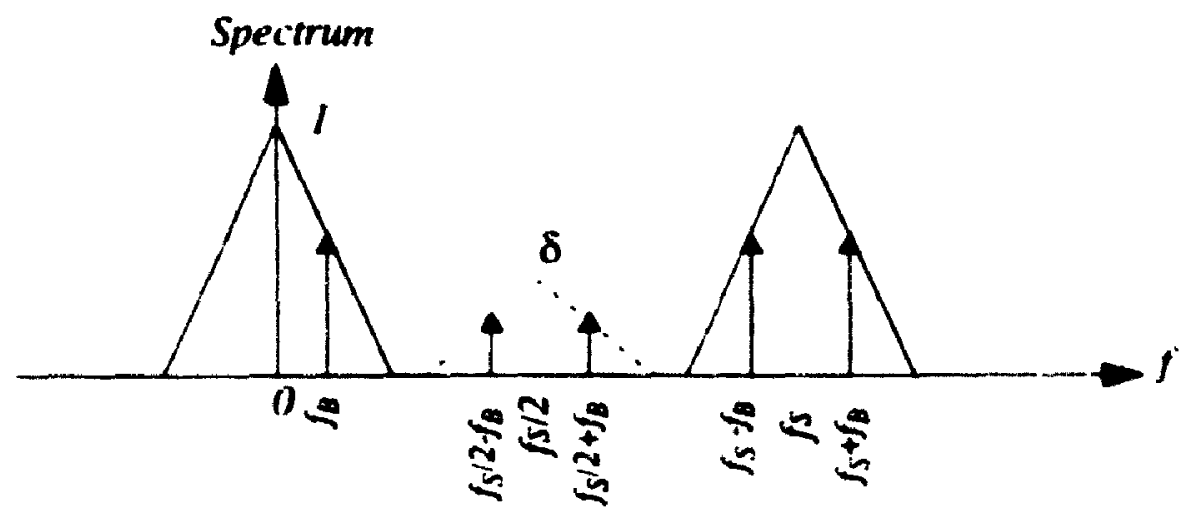

Figure 4.19: Spectrum of the input signal $V_{\text {in }}$ (solid line) and the attenuated odd samples of the input signal (dotted line)

In the frequency domain, a sampled input signal $V_{i n}$ with a frequency $f_{b}$ will have a periodic spectrum with the signal appearing at $n f_{s} \pm f_{B}$. The odd sequence of the signal has a sampling frequency of $f_{s} / 2$ and thus attenuated images appear at $n\left(f_{s} / 2\right) \pm f_{B}$.

Non-uniform sampling due to uneven $\phi_{1}$ and $\phi_{2}$ phases has a similar effect [Yang 94 |. If phase $\phi_{1}$ is longet by an amount $\tau$ compared to the $\phi_{2}$ phase, then we can write $1+\delta=e^{-s \tau}=1-s \tau$, so $\delta=-s \tau$.

\subsection{A SC Bandpass Sigma-Delta Modulator}

A fully differential SC resonator using two sample-and-hold delay cells in a negative feedback loop is illustrated in Figure 4.20.

In this circuit, if all the capacitors have the same size the transfer function of the resonator is

$$
H_{R}(z)=\frac{V_{\text {od }}}{V_{i d}}(z)=\frac{z^{-2}}{1+z^{-2}}
$$

Poles of this resonator are at $z= \pm j$. Capacitor mismatch and finite opamp gain cause errors in the transfer function.

If the gain error in the unity gain delay cell due to capacitor mismatch is $\delta$. the resulting transfer function of the resonator will be 


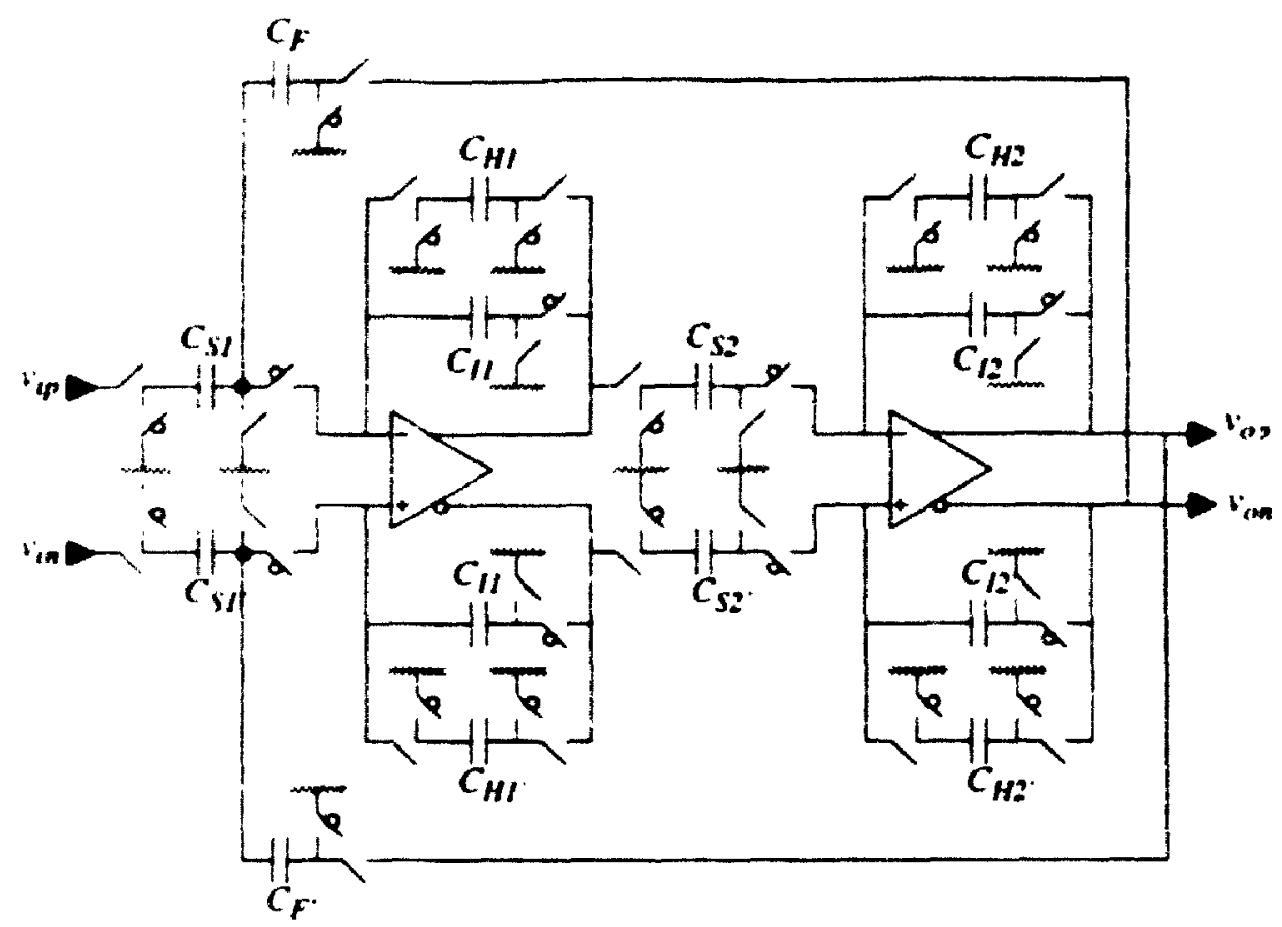

Figure 4.20: A SC resonator using two delay cells

$$
H_{R}(z)=\frac{(1-\delta)^{2} z^{-2}}{1+(1-\delta)^{2} z^{-2}}
$$

The new location of poles are at $z= \pm(1-\delta) j$. Therefore, a $\delta$ percent gain error in the unity gain delay circuit will cause $\mathbf{2 \delta}$ percent change in the gain of the resonator and $\delta$ percent change in the location of the poles cf the resonator. Note that the poles of the resonator are still on the $j \omega$-axis.

In section 4.3, it was shown that finite opamp gain causes both gain error and phase error in the ideal transfer function of a SC delay circuit, and the actual transfer function is given by (4-43). Using this transfer function for the delay cell, the resonator transfer function will be

$$
H_{R}(z)=\frac{\left[\left(m_{F D 1} \cdot e^{j \theta_{F D 1}}\right) \cdot\left(m_{F D 2} \cdot e^{j \theta_{F D 2}}\right)\right] z^{-2}}{1+\left[\left(m_{F D 1} \cdot e^{j \theta_{F D 1}}\right) \cdot\left(m_{F D 2} \cdot e^{j \theta_{F D 2}}\right)\right] z^{-2}},
$$

where $m_{F D}$ and $e^{j \theta_{F D}}$ are the gain and phase error of the $i$-th delay cell. Poles of this 
resonator are

$$
z_{p 1, p 2}= \pm j \sqrt{m_{F D 1}-m_{F D 2}} \cdot e^{\frac{\left(\theta_{F D 1}+\theta_{F(D z}\right)}{2}} .
$$

Thus, resonator poles are moved inside the unit circle (because $\left.m_{F \mid) \mid} m_{F \mid l 2}<1\right)$ and have a phase lag of $\theta_{R 0}$. Using the simplitied equation of (4-12). the phase shift of the $f_{s} / 4$ resonator is

$$
\theta_{R 0}=\frac{\sqrt{2}}{2 A}\left[\left(1+C_{i n} / C_{I}\right)+\left(1+C_{i n} / C_{H}\right)\right]
$$

Example 4.4: If an $f_{s} / 4$ resonator is built using two delay cells in a n-gative feedback loop and the capacitor sizes in terms of a unit capacitor $C_{\mu}$ are $C_{\text {in }}=C_{1}=2 C_{\mu}$ and $C_{H}=C_{\mu}$, an opamp gain of $40 \mathrm{~dB}$ will cause a phase error of 11.135 radians, almost $2.2 \%$ of the nominal frequency.

A resonator with an arbitrary gain is achieved by choosing different values for the capacitors $C_{S 2}$ and $C_{12}$ in the second delay cell of the resonator of Figure 4.2(1. During $\phi_{2}$ the transfer functio. of this circuit is

$$
H_{R}(z)=\frac{\tau_{S 2}}{C_{12}} \cdot \frac{z^{-3 / 2}}{1+z^{-2}} .
$$

If $C_{12}=2 C_{S 2}$, a resonator with gain of 0.5 is obtained which is useful for the implementation of the fourth-order bandpass $\mathbf{\Sigma}$ modulator shown in Figure 4.1 .

A fourth-order SC $\Sigma \Delta$ modulator is achieved by cascading two resonators and a quantizer in a feedback loop as shown in Figure 4.21.

In this circuit all the capacitors are identical $\left(C_{u}\right)$ except for the four marked by asterisks which have a value of $2 C_{u}$. These four capacitors scale the gain of the resonators to the required value of 0.5 .

Speed of operation is determined by the closed-loop time constant of the opamps, as discussed before. Worst case opamp loading for this architecture occurs for the vecund opamp (OA2) during $\phi_{1}$, and a single-ended representation of it is shown in Figure 4.22a. 


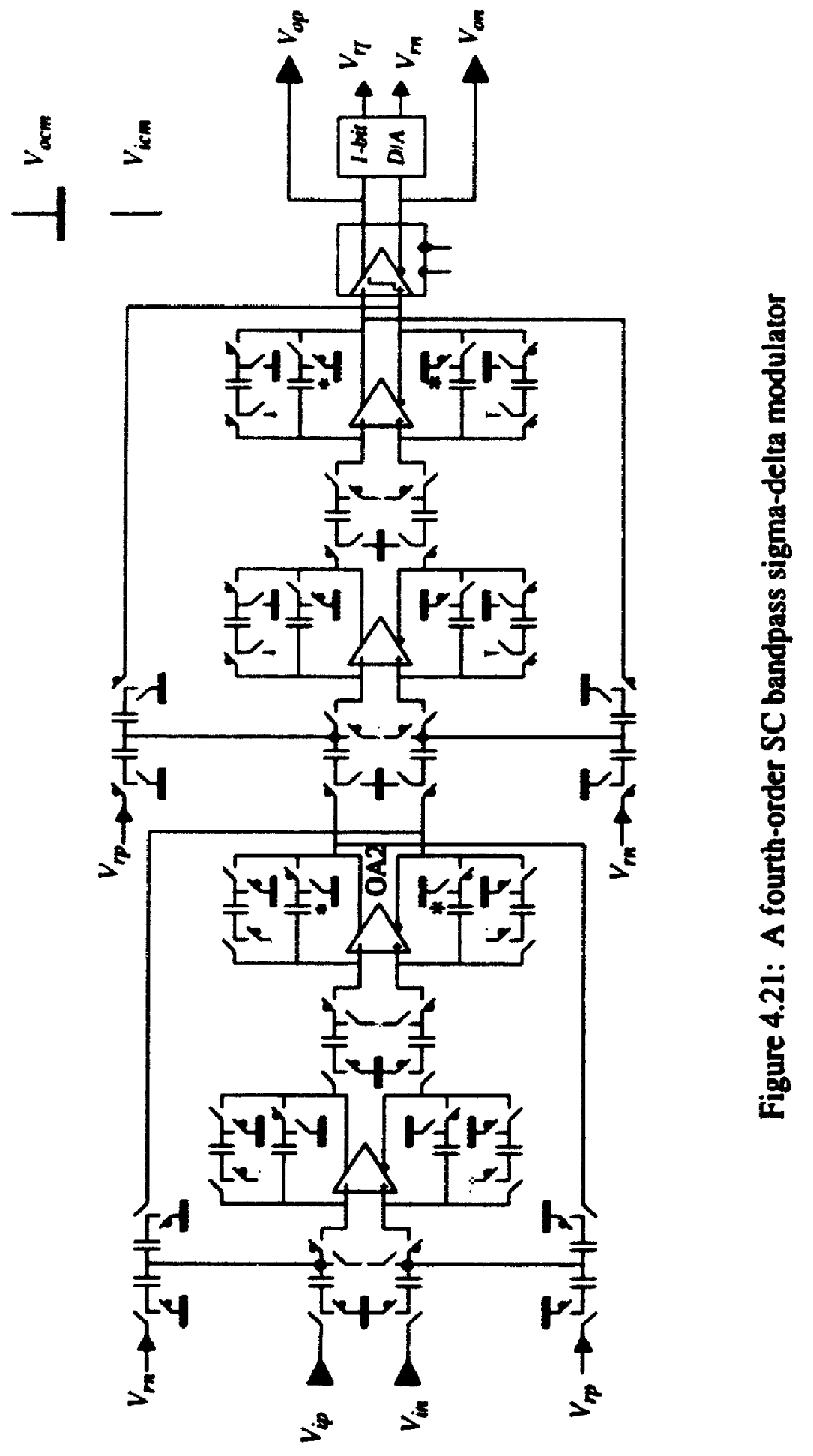


If the opamp input capacitance $C_{\text {in }}$ is assumed to be equal to the unit capacitance $C_{u}$ used in the modulator, the worst case closed-loop time constant is found to be $\tau=7 C_{u} / g_{m}$ (from equation (4-12)).

A similar analysis for the fourth-order SC bandpass $\Sigma \Delta$ reported in [Longo93] indicates that the first opamp during $\phi_{2}$ has the worst case loading, as shown in Figure $4.22 b$. The settling time constant of this structure is $9 C_{u} / g_{m}$, which is about $28 \%$ slower than the circuit presented in this work.

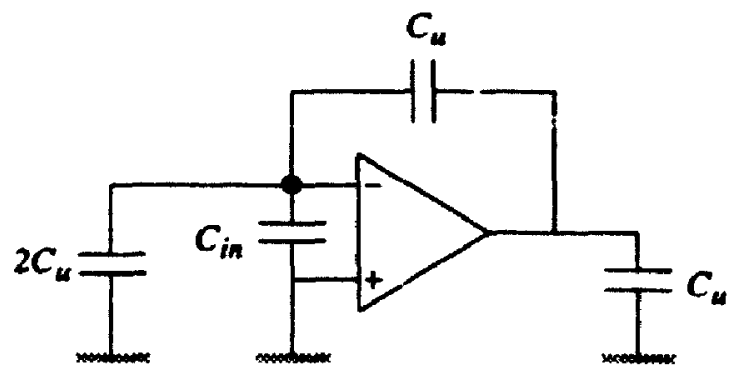

(a)

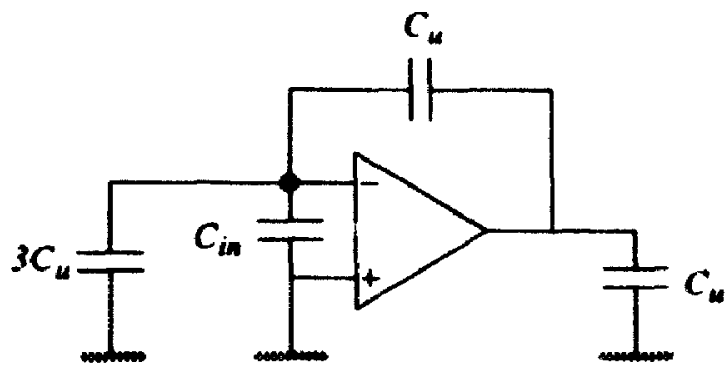

(b)

Figure 4.22: Worst case opamp loading for (a) bandpass $\Sigma \Delta$ of Figure 4.21

(b) bandpass $\Sigma \Delta$ modulator in [Longo93]

This modulator was simulated in Eldo using near ideal models for switches, capacitors, opamps, and the quantizer. The DC gain of opamps was set to $60 \mathrm{~dB}$ and switch onresistance was set to $200 \Omega$. Figure 4.23 shows the modulator output spectrum for a 24.9 $\mathrm{MHz}$ sinusoidal input signal with an amplitude of $12 \mathrm{~dB}$ below full scale. The
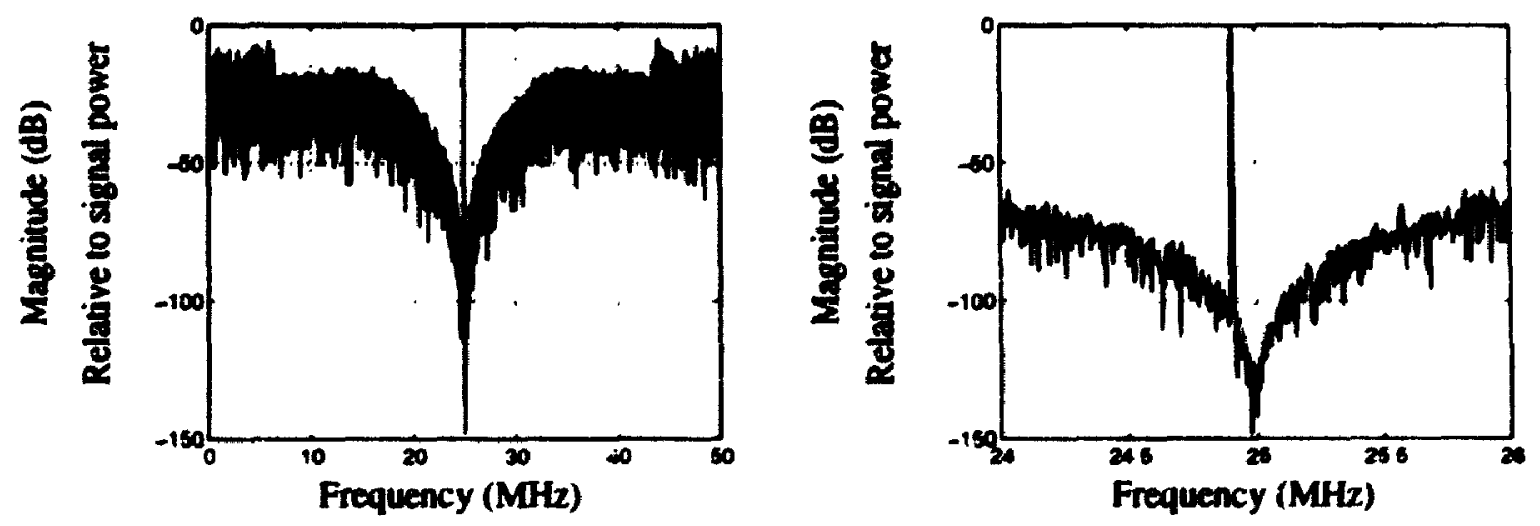

Figure 4.23: Output spectrum from Eldo simulation 
sampling rate of $100 \mathrm{MHz}$ is provided by a two-phase non-overlapping clock. Simulated SNR are $94.3 \mathrm{~dB}$ and $58.8 \mathrm{~dB}$ in $200 \mathrm{kHz}(O S R=250)$ and $1 \mathrm{MHz}(O S R=50)$ bandwidths respectively.

\subsubsection{Sources of Error}

Practical analog circuit realization of bandpass sigma-delta modulators involves errors due to device and circuit non-idealities. In the following section, some prominent sources of error, namely capacitor mismatch, finite opamp gain, and thermal noise are considered.

\section{Capacilor Mismatch}

Noise shaping is determined by the quality of the resonators used in the forward path of the modulator. Resonators used in the bandpass $\Sigma \Delta$ modulator have a gain of 0.5 and their ideal transfer function is

$$
H_{R}(z)=\frac{1}{2} \cdot \frac{z^{-2}}{1+z^{-2}} \text {. }
$$

A mismatch of $\delta$ between capacitors changes the ideal transfer function of the resonators to

$$
H_{R}(z)=\frac{1}{2} \cdot \frac{g_{0} z^{-2}}{1+p_{0} z^{-2}}
$$

where

$$
g_{0}=p_{0}=(1-\delta)^{2}
$$

As mentioned earlier, gain of the second resonator is not a cntical parameter because it is followed by a high gain quantizer. Thus, gain error in the second resonator is not going to affect the ideal behavior of the modulator. Simulations show that changing the gain of the second resonator by as much as $\pm 100 \%$ has virtually no effect on the SNR of the modulator. However, gain error in the first resonator will deteriorate the SNR of the modulator.

The ideal resonator of (4-64) has infinite gain at $f_{S} / 4$. The gain of the actual resonator of 
(4-65) at a quarter of the sampling frequency is

$$
H_{R 0}=\left.H_{R}\left(e^{j \omega T}\right)\right|_{f=f_{S} / 4}=\frac{s_{0}}{1-p_{0}}=\frac{1}{2 \delta}
$$

A lower resonator gain causes less attenuation of the quantization noise in the narrow band around $f_{S} / 4$. Performing a bandpass to lowpass transformation, using a $z^{-2} \rightarrow-z^{-1}$ change of variable on the resonator of (4-65), results in

$$
H_{I}(z)=\frac{-g_{0} z^{-1}}{1-p_{0} z^{-1}}
$$

which is a leaky integrator with DC gain of $H_{10}=g_{0} /\left(1-p_{0}\right)$. From (4-67), we can see a capacitor mismatch of $\delta$ reduces the DC gain in (4-68) to $H_{10}=1 / 2 \delta$. The effect of integrator leakage on the in-band quantization error of a second-order lowpass $\mathbf{I \Delta}$ has been analyzed in [Boser88] and is given by

$$
\frac{S_{B}}{S_{B 0}}=1+\frac{5}{\pi}\left(\frac{O S R}{H_{10}}\right)^{4}+\frac{10}{3 \pi^{2}}\left(\frac{O S R}{H_{10}}\right)^{2}
$$

Here, $S_{B}$ is the power of in-band quantization noise for a leaky integrator, and $S_{B 0}$ is the power of in-band quantization noise for a perfect integrator. If the DC gain of the integrator (or equivalently, resonator gain at $f_{s} / 4$ ) is equal to the oversampling ratio $O S R$ of the modulator, the SNR performance loss is about $1.3 \mathrm{~dB}$.

Therefore, matching between capacitors must be better than $1 /(2 \cdot$ OSR) in a fourthorder bandpass $\mathbf{\Sigma} \Delta$ modulator.

\section{Finite Opamp Gain}

Finite opamp gain causes errors in the resonant frequency and the gain of the resonator. This in rum changes the position of the notch in the noise transfer function of the bandpass $\Sigma \Delta$ modulator away from $f_{s} / 4$, and also increases the in-band quantization noise. The notch will be shifted to a lower frequency $f_{s} / 4-\Delta f$, where 


$$
\Delta f=\frac{f_{s}}{2 \pi} \cdot \theta_{R 0}
$$

In this equation, $\boldsymbol{\theta}_{R 0}$ is the phase error associated with the poles of the resonator and is given by (4-62). The frequency shift of the notch can be expressed in terms of the opamp gain and oversampling ratio of the modulator as

$$
\Delta f=\frac{\theta_{R 0}}{\pi} \cdot O S R \cdot B W
$$

For a fourth-order bandpass $\Sigma \Delta$ modulator with a quantization noise slope of about $15 \mathrm{~dB} /$ octave, a $\Delta f$ shift in the notch frequency will increase the power of in-band quantization noise by $\Delta S_{B}$ which is approximately

$$
\Delta S_{B}=\frac{15}{(B W) / 2} \cdot \Delta f=30 \cdot \frac{\theta_{R 0}}{\pi} \cdot O S R \quad \mathrm{~dB} .
$$

Substituting $\theta_{R 0}(4-62)$ in the above equation yields

$$
\Delta S_{B}=15 \frac{\sqrt{2}}{\pi} \cdot \frac{O S R}{A} \cdot\left[2+\frac{C_{i n}}{C_{l}}+\frac{C_{i n}}{C_{H}}\right]
$$

For an acceptable SNR loss, the minimum opamp gain can be found in terms of $O S R$.

Example 4.5: In the bandpass $\Sigma \Delta$ modulator of Figure 4.21, the values of different capacitors in terms of a unit capacitor $C_{u}$ are: $C_{l}=C_{i n}=2 C_{u}$ and $C_{H}=C_{S}=C_{u}$. For an oversampling ratio of $\mathbf{4 0}$ the minimum required opamp gain is $53 \mathrm{~dB}$, if a penalty of $3 \mathrm{~dB}$ SNR loss is acceptable due to notch frequency shift. Note that opamp gain ( $53 \mathrm{~dB}$ ) is $21 \mathrm{~dB}$ higher than the value of $O S R$, in decibels.

Example 4.6: In the above example, if the opamp gain is $40 \mathrm{~dB}$ and the sampling frequency is $100 \mathrm{MHz}$, the phase shift $\theta_{R O}$ is found to be 0.035 radians and from equation (4-70), the notch frequency shift is found to be $562 \mathrm{kHz}$.Thus, the resonant frequency will be at $24.44 \mathrm{MHz}$, instead of being at $25 \mathrm{MHz}$. For an $O S R=50$, the inband quantization noise power (4-72) will increase by about $16.7 \mathrm{~dB}$.

The bandpass SC $\Sigma \Delta$ modulator was simulated using opamps with DC gain of $40 \mathrm{~dB}$, and 
the parasitic capacitances at the input of the opamp was assumed to be $2 C_{u}$. The input signal is a $24.9 \mathrm{MHz}$ sinusoid with an amplitude of $12 \mathrm{~dB}$ below full scale. Figure 4.24 shows the output spectrum of the modulator. The notch frequency is shifted by about $450 \mathrm{kHz}$. Simulated SNR are $56.5 \mathrm{~dB}$ and $46.1 \mathrm{~dB}$ for bandwidth of $200 \mathrm{kHz}$ and $1 \mathrm{MHz}$ respectively.
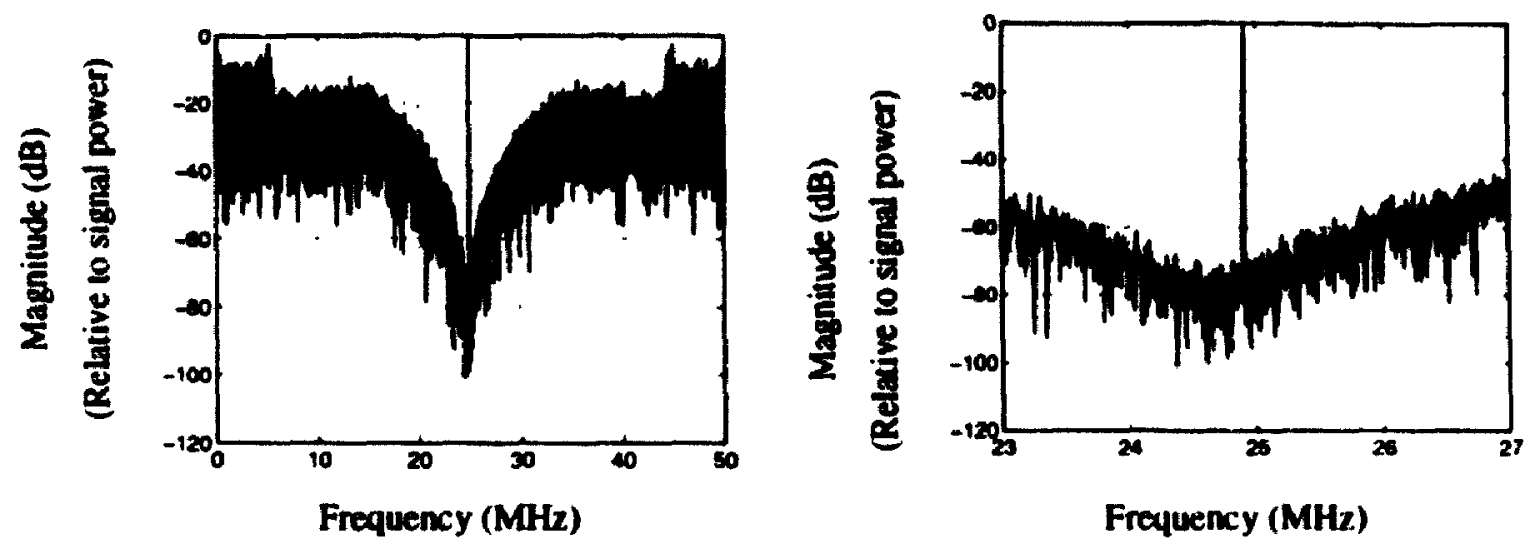

Figure 4.24: Output spectrum of the fourth-order bandpass $\Sigma \Delta$ modulator with an opamp gain of $40 \mathrm{~dB}$ and opamp input capacitance of $\mathrm{Cin}=2 \mathrm{Cu}$

If we neglect the phase error, the effect of finite opamp gain on the transfer function of a resonator is similar to capacitor mismatch and the actual transfer function of the resonator will be like equation (4-65), with $g_{0}$ and $p_{0}$ being

$$
g_{0}=p_{0}=1-\frac{1}{A}\left(\frac{1}{\beta_{Q 1,1}}+\frac{1}{\beta_{Q 2,1}}+\frac{1}{\beta_{\$ 1,2}}+\frac{1}{\beta_{\$ 2,2}}\right)
$$

where, $\beta_{\phi j, i}$ is the feedback factor of the $i$ th delay circuit during the $\phi_{j}$ th clock phase. If the feedback factors are comparable and assumed to be equal to $\beta$. the above equation is simplified to

$$
g_{0}=p_{0}=1-\frac{4}{A \beta}
$$

The gain of the resonator at its resonant frequency is

$$
\left.H_{R 0}\left(e^{j \omega T}\right)\right|_{f=f_{s} / 4}=\frac{g_{0}}{1-p_{0}} \approx \frac{A \beta}{4}
$$


The in-band quantization error as a function of resonator gain can be expressed by an equation similar to (4-69). Therefore the minimum required opamp DC gain for this double-resonator $S C$ bandpass $\Sigma \triangle$ modulator is $A_{\min (b p)}=4(O S R) / \beta$.

In a SC integrator, using an opamp with DC gain of $A$ and feedback factor of $\beta$, the integrator $D C$ gain is $H_{0}=A \beta$. Thus, the minimum required opamp DC gain in a lowpass $\Sigma \Delta$ modulator is $A_{\min (l p)}=O S R / \beta$. Note that for a similar SNR performance, the opamp used in the sample-and-hold based fourth-order bandpass $\Sigma \Delta$ modulator requires $12 \mathrm{~dB}$ higher DC gain than the opamp used in the second-order lowpass $\Sigma \Delta$ modulator.

Figure 4.25 shows the SNR loss as a function of $O S R / H_{R 0}$. From this figure, one can find the requirements on capacitor matching and/or opamp DC gain.

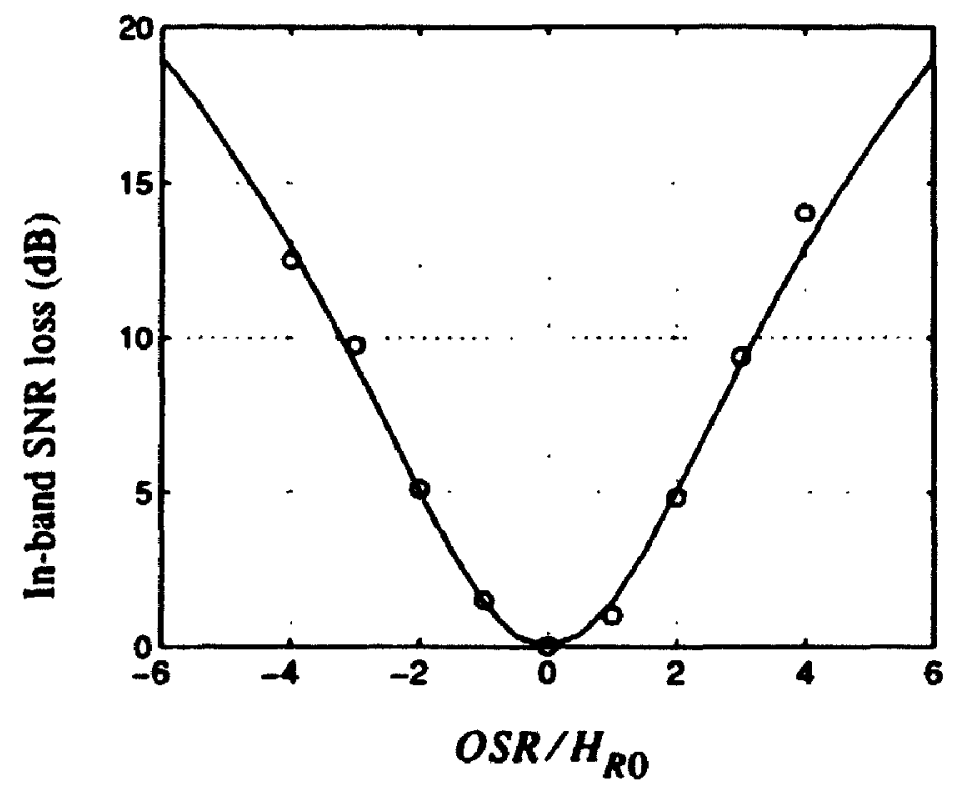

Figure 4.25: SNR loss versus $O S R$ / $H_{R O}$ from analytical result (solid line) and from simulation results (o points).

In the bandpass $\Sigma \Delta$ modulator of Example 4.5, if the oversampling ratio is 40 , a capacitor matching accuracy of at least $1.25 \%$ is needed for a SNR loss of $1.5 \mathrm{~dB}$. Calculating the $\beta$ during each phase for the two opamps and using equations (4-74) and (4-76), $H_{R 0}$ is found to be equal to $A / 16$. Thus, the minimum opamp DC gain is $56 \mathrm{~dB}$ for the same SNR loss, i.e. $1.5 \mathrm{~dB}$. 


\section{Noise}

Thermal noise generated by various switches and the two opamps in the first resonator is going to limit the accuracy of the bandpass $\Sigma \Delta$ modulator. Thermal noise generated in the second stage undergoes a second-order band-reject noise shaping. and thus its effect is not critical.

Using the results obtained earlier in this chapter on the analysis of the noise in a SC amplitier, the total input referred thermal noise power of the tirst stage of the single-ended $S C$ bandpass $\Sigma \Delta$ modulator in Figure 4.21 is

$$
\nabla_{n T}^{2}=\frac{14.75 k T}{C_{n}}
$$

In this calculation, the input capacitance of the opamp is assumed to be comparable to the unit capacitor, i.e. $C_{i n}=C_{u}$. The total noise power for a fully differential SC circuit is twice as large as (4-77). The in-band noise power is reduced due to the oversampling process and is given by:

$$
\left.\nabla_{n T}^{2}\right|_{\text {in-band }}=\frac{29.5}{O S R} \cdot \frac{k T}{C_{u}}
$$

\subsection{A Double-Sampled SC Bandpass Sigma-Delta Modulator}

A double-sampled SC resonator is obtained by cascading two double-sampled delay circuits as shown in Figure 4.26. The ideal transfer function of this circuit is

$$
H(z)=\frac{C_{l}}{C_{S 1}} \cdot \frac{z^{-2}}{1+z^{-2}}
$$

In this configuration, capacitance mismatch (between $C_{1}$ and $C_{S 1}$ ) causes a gain error on the input signal $v_{i d}=v_{i p}-v_{i n}$ that is added to the feedback signal using a two-capacitor sample-and-hold architecture. If the error due to capacitor mismatch is $\delta$, the transfer function of the double-sampled resonator will be

$$
H(z)=(1+\delta) \frac{C_{1}}{C_{s 1}} \cdot \frac{z^{-2}}{1+z^{-2}} .
$$




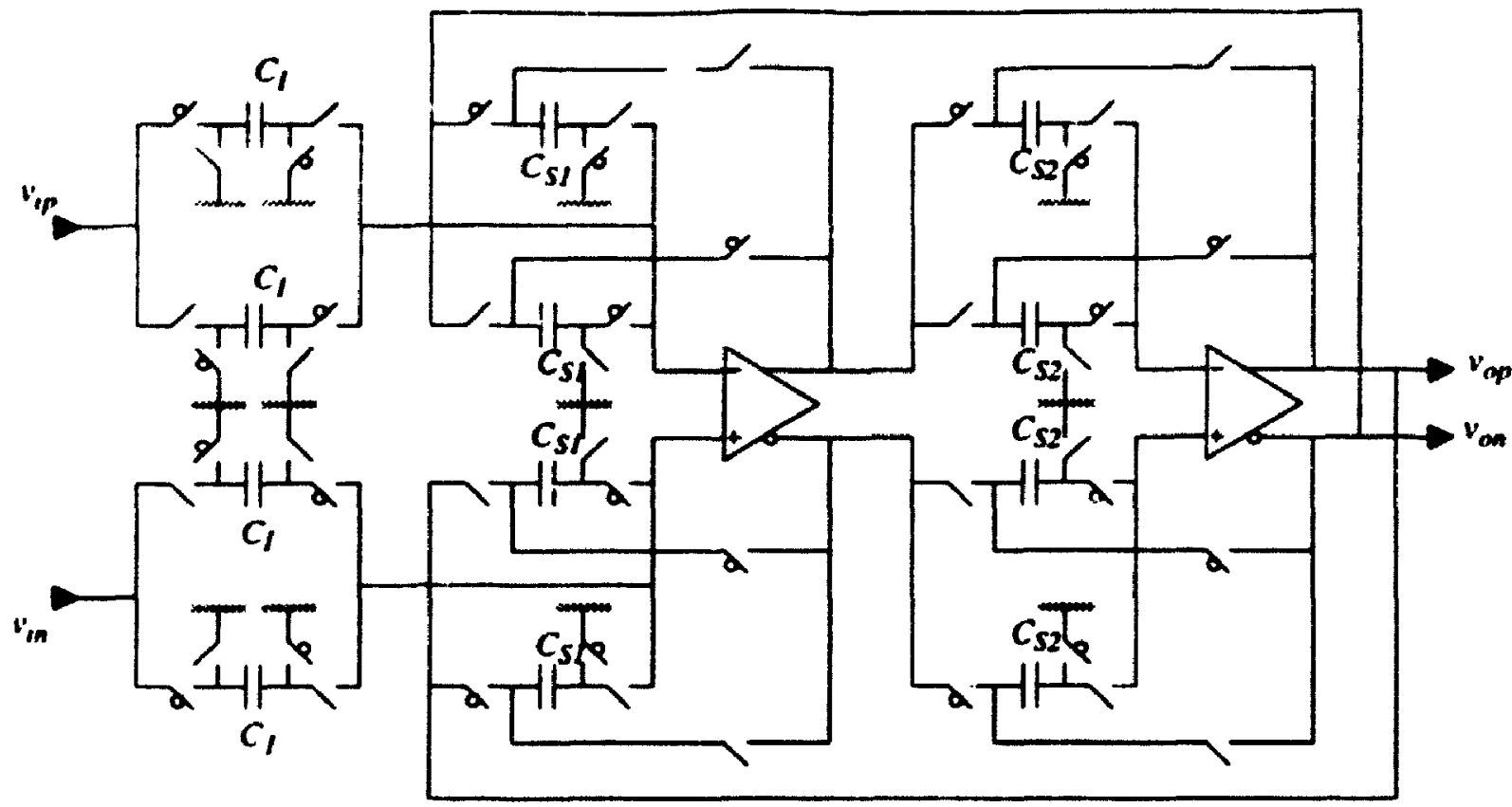

Figure 4.26: A double-sampled SC resonator

Therefore, the location of resonator poles is not affected by the capacitor mismatch. However, finite opamp gain ( $A$ ) causes errors in the ideal transfer function of a doublesampled SC delay circuit (given by (4-50)) and the transfer function of a double-sampled resonator becomes

$$
H_{d R}(z)=\frac{C_{l}}{C_{S 1}} \cdot \frac{\left[\left(m_{d D 1} \cdot e^{j \theta_{D 1}}\right) \cdot\left(m_{F D 2} \cdot e^{j \theta_{d D 2}}\right)\right] z^{-2}}{1+\left[\left(m_{d D 1} \cdot e^{j \theta_{d D 1}}\right) \cdot\left(m_{d D 2} \cdot e^{j \theta_{d D 2}}\right)\right] z^{-2}}
$$

Poles of this resonator are

$$
z_{p 1, p 2}= \pm j \sqrt{m_{d D 1} \cdot m_{d D 2}} \cdot e^{j \frac{\left(\theta_{d D 1}+\theta_{d D_{2}}\right)}{2}} .
$$

The poles are inside the unit circle, close to the intersection of $j \omega$-axis and the unit circle. The phase error of the poles of this resonator in radians is

$$
\theta_{d R}=-\frac{\sin \omega T}{2 A}\left[\frac{C_{i n 1}}{C_{S 1}}+\frac{C_{i n 2}}{C_{S 2}}\right] .
$$


Both magnitude and phase errors are inversely proportional to the DC gain of the opamp. A double-sampled double-resonator $\mathrm{SC}$ bandpass $\Sigma \Delta$ modulator is constructed using two double-sampled resonators and a quantizer in a feedback loop, as shown in Figure 4.27.

All the capacitors are unit size capacitors $\left(C_{u}\right)_{0}$ except for the eight marked by asterisks which have a value of $2 C_{u}$, and are made of two unit size capacitors in parallel. The gain of resonators is set by the eight capacitors to the required value of 0.5 .

The functionality of this double-sampled SC bandpass $\mathbf{\Sigma}$ modulator was verified in Eldo using ideal components. The on-resistance of the switches was set to $200 \Omega$ and the DC gain of opamps was assumed to be $60 \mathrm{~dB}$. Figure 4.28 shows the output spectrum of the modulator for a sinusoid input signal of $50.1 \mathrm{MHz}$. The amplitude of the signal was $12 \mathrm{~dB}$ below full scale and the clock frequency was $100 \mathrm{MHz}$.
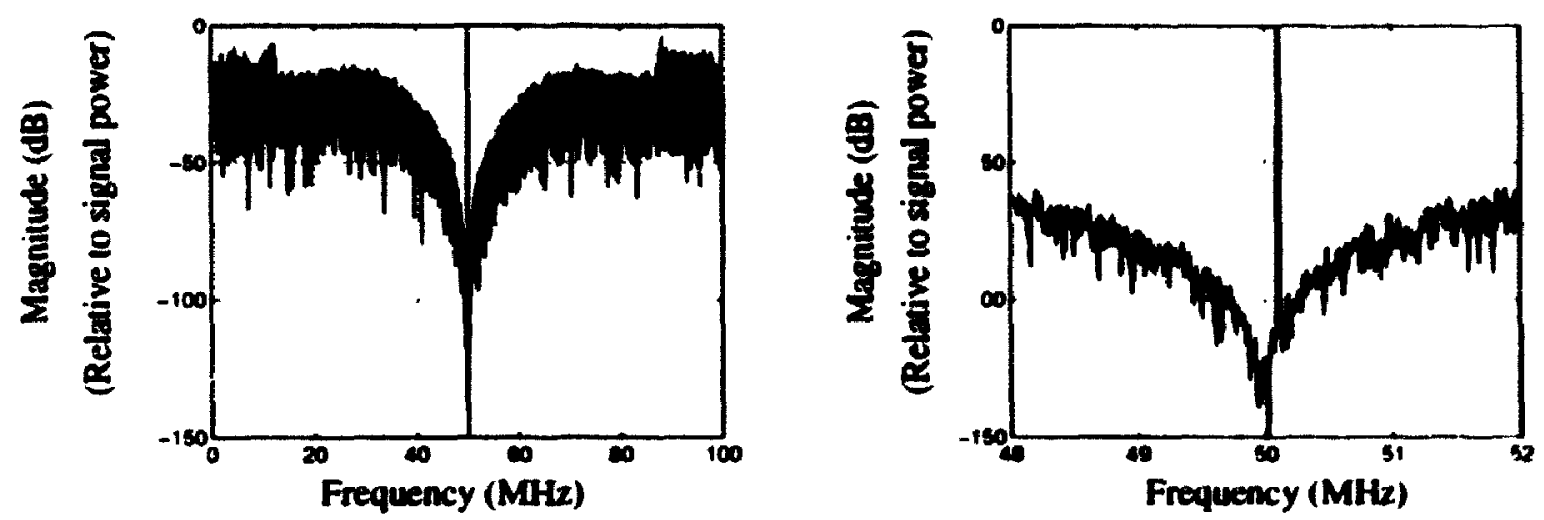

Figure 4.28: Output spectrum from Eldo simulation

Simulated SNR is about $108 \mathrm{~dB}$ and $72.8 \mathrm{~dB}$ for bandwidths of $200 \mathrm{kHz}$ and $1 \mathrm{MHz}$ respectively. For the same clock frequency and signal bandwidth, the oversampling ratio of this modulator is twice as large as the single-sampled modulator. Thus, the SNR of this modulator is $15 \mathrm{~dB}$ higher than that of a single-sampled counterpart.

\subsubsection{Sounces of Error}

Performance of the double-sampled $\Sigma \Delta$ modulator is affected by circuit non-idealities. In the following, effects of some prominent non-idealities such as capacitor mismatch. finite opamp gain, and noise on the SNR of the double-sampled $\Sigma \Delta$ modulator are described. 
$-\frac{5}{5}=$

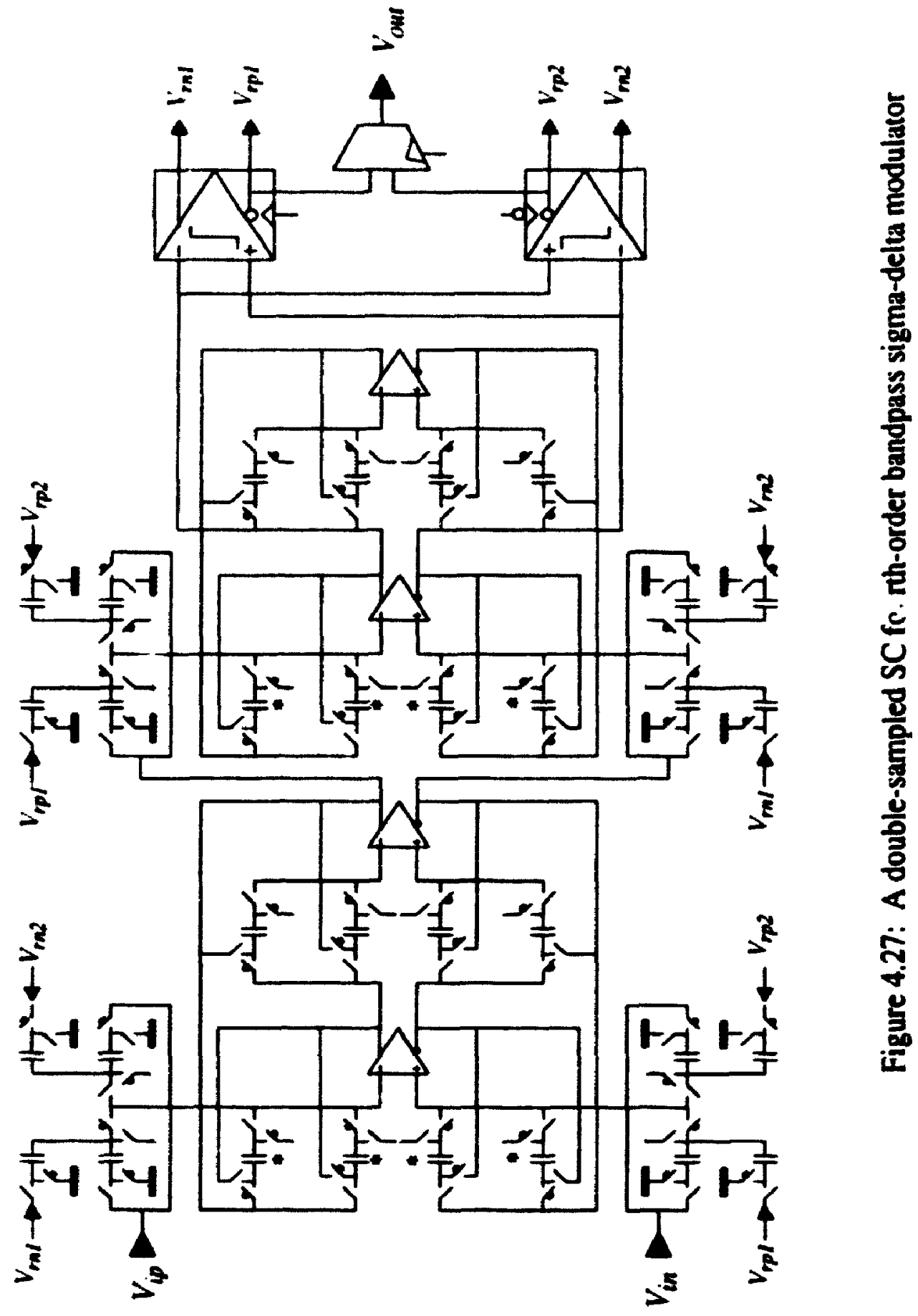




\section{Capacitor Mismatch}

As mentioned before. double-sampled SC circuits are sensitive to path mismatch and any mismatch between the two channels will produce image problems. Mismatch in the second stage of the modulator is noise shaped (second-order noise-shaping) and will not cause a noticeable image signal. Simulations were carried out using different capacitor mismatches to verify the above argument. A path mismatch of $5 \%$ in the second stage will produce an image $45 \mathrm{~dB}$ below full scale. However, mismatch in the tirst stage of the modulator is critical and must be avoided.

A path mismatch of $1 \%$ on the input in the first stage of the modulator will produce an image signal which is only $40 \mathrm{~dB}$ below the signal. Figure 4.29 shows the output spectrum of the modulator with a capacitor mismatch of $1 \%$ between the input sampling capacitors (during $\phi_{1}$ and $\phi_{2}$ ). Using layout techniques such as common-centroid. good
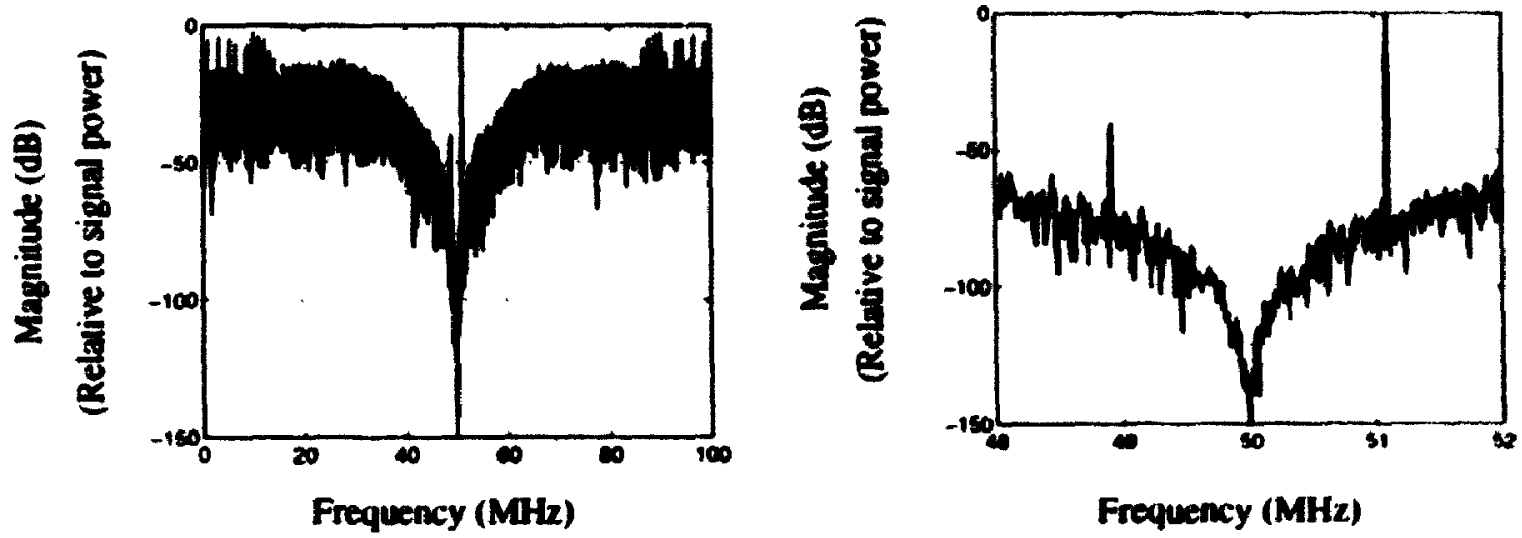

Figure 4.29: Output spectrum of the double-sampled bandpass sigma-delta modulator with $1 \%$ capacitor mismatch between the two paths. Note that the image power is $40 \mathrm{~dB}$ below the signal power.

capacitor matching -in the order of $0.1 \%$ - can be achieved. A capacitor mismatch of $0.1 \%$ will reduce the power of image signal to about $60 \mathrm{~dB}$ below the signal power. This kind of accuracy, ten bits, is acceptable for some high-speed wide-band applications, such as rable modems and PCS basestations.

Other methods of overcoming mismatch error, which have previously been applied to lowpass $\Sigma \Delta$ modulators, include a four-phase clocking scheme proposed by Ribner 
[Ribner91], a switching scheme that converts the capacitive mismatch into an additive out of band noise [Hurst92] [Burmas95], and a method of using bilinear integrator [Yang94].

\section{Finite Opanp Gain}

Finite opamp gain causes both phase and gain errors in the ideal transfer function of a double-siampled resonator. The resonant frequency of the double-sampled SC resonator due to tinite opamp gain is shifted by an angle $\theta_{d R 0}$ from the $j \omega$-axis. If the same opamp is used in both delay cells and $C_{S 1}=C_{S 2}=C_{S}$, using (4-83) the phase error in the location of the poles of the double-sampled SC resonator is

$$
\theta_{d R 0}=\frac{C_{\text {in }}}{C_{S}} \frac{1}{A} .
$$

Taus, increasing the opamp DC gain and/or reducing the opamp input capacitance reduces the phase error. The frequency of the notch filter (for noise transfer function) will be shifted by $\Delta f$ where

$$
\Delta f=\frac{2 f_{S}}{2 \pi} \cdot \theta_{d R 0}=\frac{C_{\text {in }}}{C_{S}} \cdot \frac{O S R}{A} \cdot \frac{2 B W}{\pi}
$$

In a fourth-order bandpass $\Sigma \Delta$ modulator, the quantization noise slope is $15 \mathrm{~dB} /$ octave. reaching its minimum at the resonant frequency. If the resonant frequency is shifted by $\Delta f$, the in-band noise will be increased by approximately

$$
\Delta S_{B}=\frac{15}{(B W) / 2} \cdot \Delta f=\frac{60}{\pi} \cdot \frac{C_{i n}}{C_{S}} \cdot \frac{O S R}{A}
$$

For a $1.5 \mathrm{~dB}$ penalty in SNR, the opamp gain must be larger than the oversampling ratio by a factor of 12.7 (about $22 \mathrm{~dB}$ ), if the input capacitance of the opamp is equal to the sampling capacitor.

\section{Noise}

The RC thermal noise associated with the different capacitors and switches in a doublesampled $\Sigma \Delta$ is less than the simple SC $\Sigma_{\Delta}$ modulator because fewer capacitors are involved. Using the result of noise analysis in section 4.2, the total input referred thermal 
noise associated with switches and opamp in a fully differential double-sampled SC $\mathrm{E}$ modulator is

$$
r_{n T}^{2}=\frac{17.8 k T}{C_{n}}
$$

Compared to the total noise of a simple SC bandpass $\Sigma \Delta$ modulator, this is smaller by a factor of 1.65 times. The in-band input referred thermal noise will the

$$
\nabla_{n T}^{2}=\frac{17.8}{O S R} \cdot \frac{k T}{C_{u}}
$$

\subsection{Implementation}

Fourth-order bandpass $\Sigma \Delta$ modulators in Figure 4.21 and Figure $\mathbf{4 . 2 7}$ were designed and fabricated in a $0.5 \mu \mathrm{m}$ double-poly CMOS process (HCMOS5a from SGS-Thomson). Chip microphotographs of the simple SC bandpass and the double-sampled bandpass modulator are shown in Figure 4.30 and Figure 4.31 respectively. The active chip area of both modulators is about $1.1 \mathrm{~mm}^{2}$.

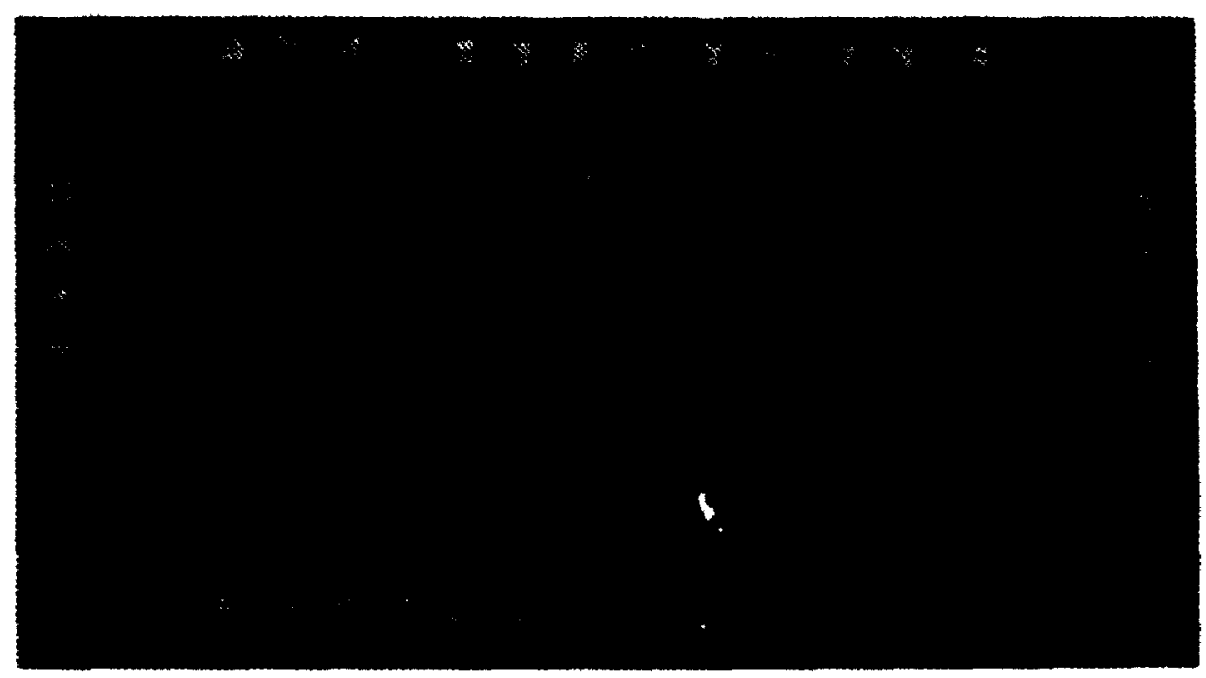

Figure 4.30: Chip microphotograph of the fourth-order SC bandpass sigma-delta modulator

HCMOS5a technology offers MOSFETs with a channel length of $0.5 \mu \mathrm{m}$ and a high quality poly-to-poly capacitor, with a capacitance per unit area of $1.1 \mathrm{fF} / \mathrm{\mu m}^{2}$. Some 


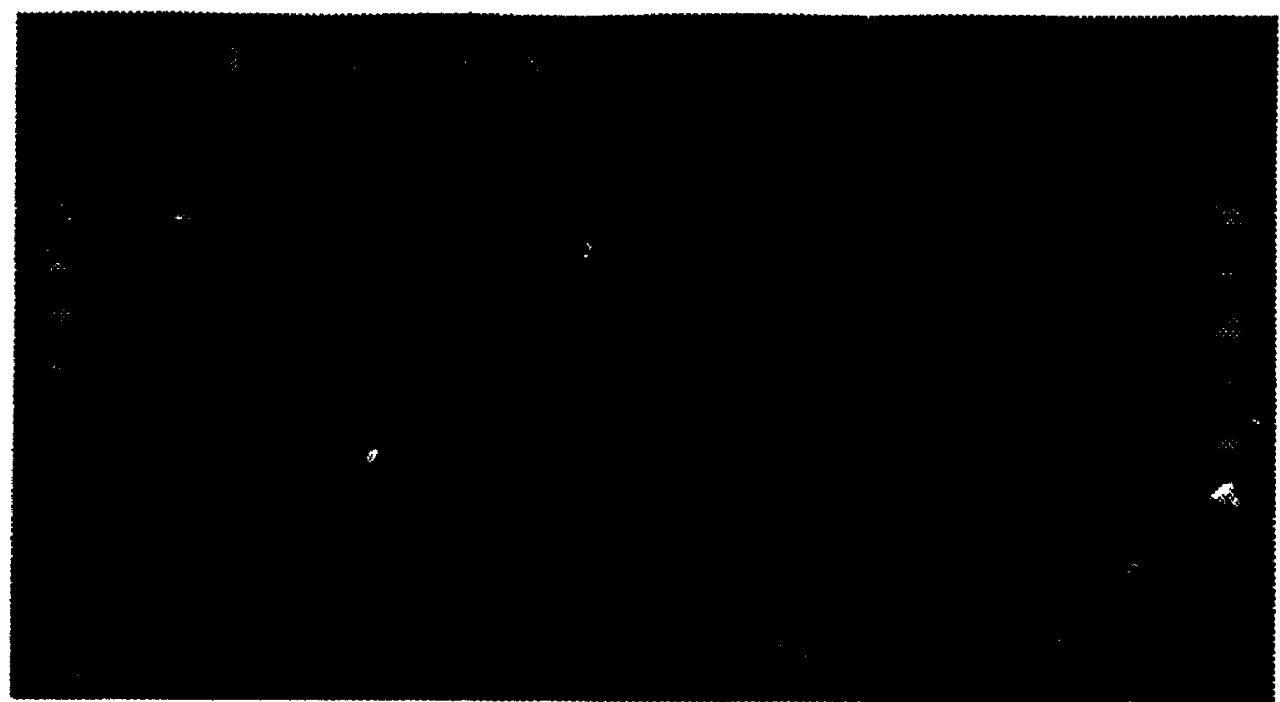

Figure 4.31: Chip microphotograph of the fourth-order double-sampled SC bandpass sigma-delta modulator

MOSFET parameters in this technology are listed in Table 4.1.

\begin{tabular}{|c|c|c|}
\hline Parameters & nMOSFET & pMOSFET \\
\hline \hline$V t(10 \mu \mathrm{m} / 0.5 \mu \mathrm{m})$ & $600 \mathrm{mV}$ & $600 \mathrm{mV}$ \\
\hline Isat $\left(@ V_{g}=V d=3.3 \mathrm{~V}\right)$ & $0.33 \mathrm{~mA} / \mu \mathrm{m}$ & $0.15 \mathrm{~mA} / \mu \mathrm{m}$ \\
\hline$\mu C_{o x}$ & $120 \mu \mathrm{A} / \mathrm{V}^{2}$ & $31 \mu \mathrm{A} / \mathrm{V}^{2}$ \\
\hline
\end{tabular}

Table 4.1: Some MOSFET parameters in HCMOS5a process

The main objective of the design was to demonstrate high-speed SC capabilities in submicron CMOS technologies. In [Singor94], it is shown that SC circuits operating at $40 \mathrm{MHz}$ are feasible in a $0.8 \mu \mathrm{m}$ BiCMOS process. Here, the target clock frequency was set to be $80 \mathrm{MHz}$. Therefore the IF frequency was at $20 \mathrm{MHz}$.

An interesting application of the bandpass $\Sigma \Delta$ modulator is in $A D D$ conversion of narrowband ( $200 \mathrm{kHz}-1 \mathrm{MHz}$ ) signals in high-speed RF modems. The required dynamic range of these A/D converter depends on the specific system architecture. Typically, a SNDR of about $60 \mathrm{~dB}$ is needed.

The target specification for the bandpass $\Sigma \Delta$ modulator is given in Table 4.2. 


\begin{tabular}{|c|c|}
\hline Parameter & Specification \\
\hline \hline IF frequency & $20 \mathrm{MHz}$ \\
\hline Bandwidth & $1 \mathrm{MHz}$ \\
\hline SNDR & $\sim 60 \mathrm{~dB}$ \\
\hline Power & $<100 \mathrm{~mW}$ \\
\hline
\end{tabular}

Table 4.2: Target specification for the bandpass $\Sigma \Delta$ modulator

The oversampling ratio of this modulator is 40 . An ideal fourth-order bandpass $\Sigma \Delta$ modulator with an OSR of 40 can achieve a maximum SNR of $62 \mathrm{~dB}$.

To achieve high speed at moderate power, the unit capacitors are chosen to be as low as $300 \mathrm{fF}$. The total in-band $k T / C$ noise of the single-sampled modulator (4-78) with $O S R=40$ is calculated to be less than $-76 \mathrm{~dB}$ relative to a $2 \mathrm{~V}$ peak-to-peak signal. In the following, the design of switches, opamp, and comparator circuits is described.

\section{Switch Design}

In stray insensitive SC circuits, typically the signal is sampled on a capacitor by means of two turned-on MOSFET switches. For example in the SC amplifier of Figure 4.5a. during $\phi_{1}$ the input is sampled on $C_{s}$ through $S 1$ and $S 2$ switches. The on-resistance of th switches along with the samplirg capacitor constitutes an RC circuit. The sampled signal on $C_{s}$ has the following exponential behovior:

$$
v_{C_{1}}=v_{i p}\left(1-e^{-i / R C_{1}}\right)
$$

Therefore, at the end of the sampling phase ( $\left.t=T_{s} / 2\right)$, the input voltage is sampled on $C_{s}$ with a settling error given by the term in the parentheses of (4-89). For a $0.1 \%$ settling error in 4.5 ns (the sampling period), the on-resistance of each MOSFET switch must be less than $560 \Omega$. For a better fixed charge cancellation, equal size pMOSFET and nMOSFET switches are utilized. Eldo simulations were carried out to find the exact value of the MOSFET aspect ratios. Figure 4.32 shi ws the simulated on-resistance of a parallel nMOSFET and pMOSFET switches with $W / L=40 \mu \mathrm{m} / 0.5 \mu \mathrm{m}$. The worst case onresistance is about $333 \Omega$. 


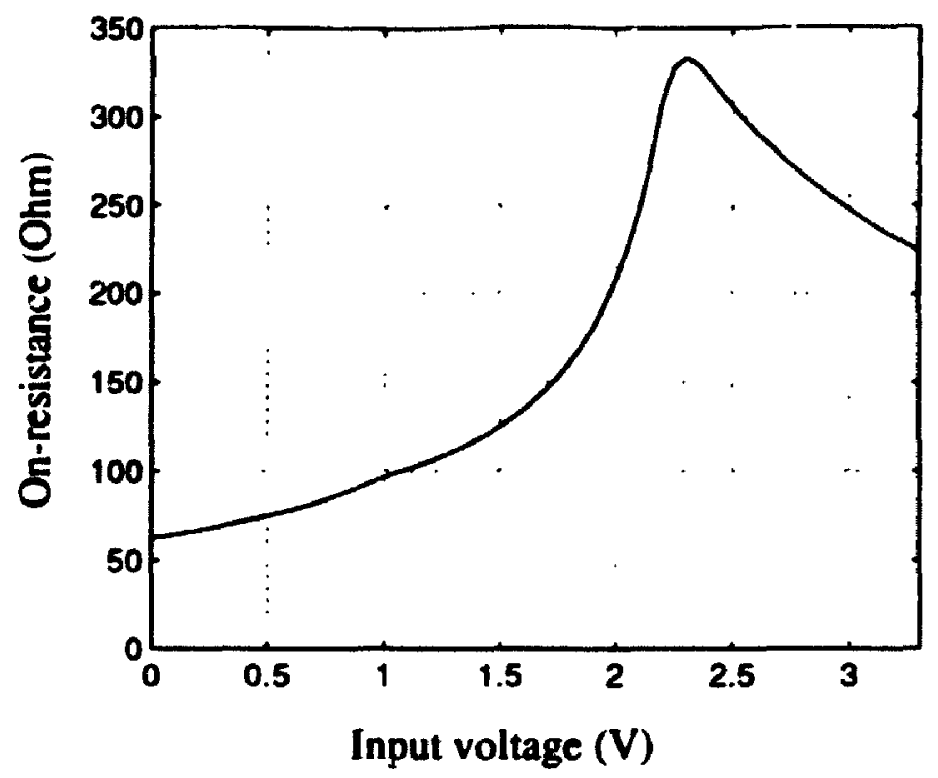

Figure 4.32: Transmission gate switch on-resistance vs. signal level

\section{Operational Transconductance Amplifier Design}

For high-speed SC operation, the unity gain frequency of the opamp must be 5 to 10 times greater than the clock frequency [Gregorian86]. For a $0.1 \%$ settling error in half a clock period, the unity gain frequency of the opamp (with the worst case capacitive load) must be 7 times greater than the clock frequency. The worst case opamp load in our design is about $1 \mathrm{pF}$. Thus, for $80 \mathrm{MHz}$ clock frequency the unity-gain frequency of the opamp driving a $1 \mathrm{pF}$ load must be greater than $560 \mathrm{MHz}$.

The required DC gain of the opamp depends on the oversampling ratio of the modulator. In section 4.4.1, it was shown that for a $3 \mathrm{~dB}$ penalty in SNR at an oversampling ratio of 40, the opamp DC gain must be at least $53 \mathrm{~dB}$.

For large input signals, slewing response of the opamp determines the maximum speed of operation. During the non-overlapping phase, the opamp is in an open-loop configuration and its output levels are typically clipped to a voltage close to the rails. A fas! slew rate of $1 \mathrm{~V} / \mathrm{ns}$ is chosen to bring the output voltages quickly to the workable levels of $1.4 \mathrm{~V}$ and 2.4 V. For the worst case opamp load of $1 \mathrm{pF}$, a bias current of $1 \mathrm{~mA}$ at each output stage is required.

A single stage cascode opamp (also called a telescopic opamp [Senderow94]) is used to 
achieve the high speed and adequate DC gain needed for the opamp. The schematic of a fully differential cascode opamp designed to fulfill these requirements is shown in Figure 4.33. A continuous time common-mode feedback circuit sets the output common mode to the desired value. Resistors in the common-mode feedback circuit have a high value of $68 \mathrm{k} \Omega$ to ensure the opamp DC gain does not drop below $54 \mathrm{~dB}$. The capacitors in the common mode feedback circuit have a value of $400 \mathrm{fF}$.
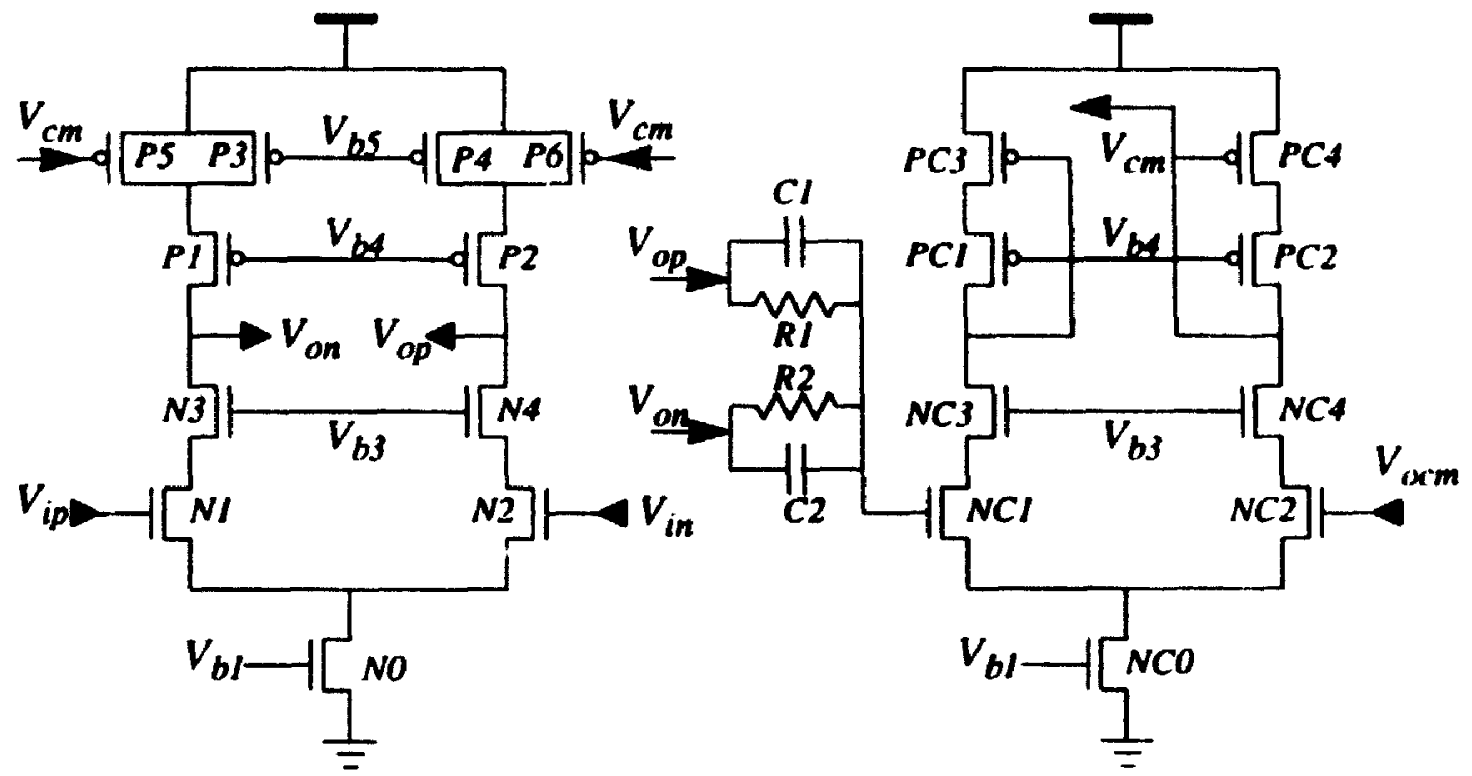

Figure 4.33: Fully differential cascode opamp

Dimensions of all the transistors used in the opamp are listed in Table 4.3, and transistor sizes for the common mode feedback are given in Table 4.4.

\begin{tabular}{|c|c|c|c|c|c|c|c|c|c|c|c|}
\hline MOSFET & NO & NI & N2 & N3 & N4 & PI & P2 & P3 & P4 & P5 & Po \\
\hline \hline$W(\mu \mathrm{m})$ & 400 & 200 & 200 & 200 & 200 & 360 & 360 & 240 & 240 & 120 & 120 \\
\hline$L(\mu \mathrm{m})$ & 0.7 & 0.5 & 0.5 & 0.7 & 0.7 & 0.7 & 0.7 & 0.7 & 0.7 & 0.7 & 0.7 \\
\hline
\end{tabular}

Table 4.3: Transistors sizes used in the opamp

\begin{tabular}{|c|c|c|c|c|c|c|c|}
\hline MOSFETS & NCO & NCI & NC2 & $P C 1$ & $P C 2$ & $P C 3$ & $P C 4$ \\
\hline \hline$W(\mu \mathrm{m})$ & 133 & 80 & 80 & 120 & 120 & 120 & 120 \\
\hline$L(\mu \mathrm{m})$ & 0.7 & 0.5 & 0.5 & 0.7 & 0.7 & 0.7 & 0.7 \\
\hline
\end{tabular}

Table 4.4: Transistor sizes used in the CMFB circuit 
This opamp was simulated in Eldo using SPICE level 3 MOSFET models. Output conductance of transistors is poorly modeled in SPICE level 3 models. Therefore, simulations typically predict an optimistically high DC gain for the opamp. A safety margin of $12 \mathrm{~dB}$ was added to the required simulated opamp DC gain (not including the loading by resistor divider in the CMFB circuit) because of this modelling error. The

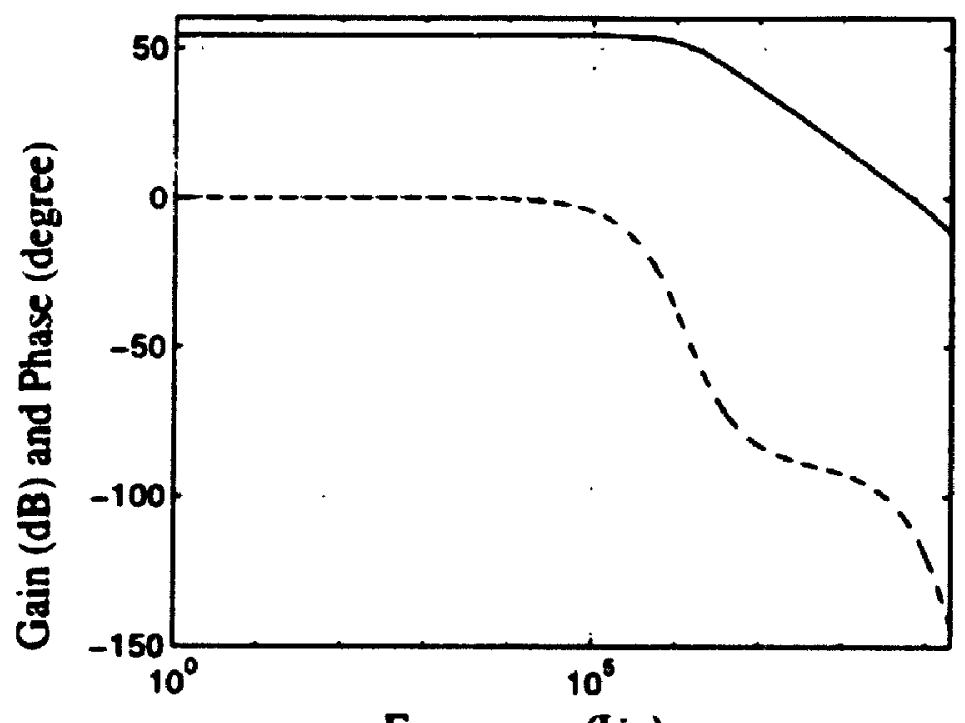

Frequency (Hz)

Figure 4.34: Simulated open loop gain (solid line) and phase (dotted line) of the cascode opamp for 1 pF load capacitance

opamp was simulated by itself and the DC gain was about $66 \mathrm{~dB}$. The resistor divider in the CMFB circuit reduces the DC gain to $54 \mathrm{~dB}$.

Simulated characteristics of the complete opamp at 3.3V power supply, $2 \mathrm{~mA}$ bias current and $1 \mathrm{pF}$ output load are summarized in Table 4.5.

The settling time of the delay circuit was simulated in Eldo using the schematic of Figure 4.35. In the modulator of Figure 4.21, the worst case settling time happens when $C_{S}=2 C_{u}, C_{H}=C_{u}$, and $C_{L}=C_{u}$. Setting time to $0.1 \%$ of the full scale in this configuration is about $4.9 \mathrm{~ns}$, as shown in Figure 4.36.

\section{Comparator}

In a $\Sigma \Delta$ modulator circuit, the required specification of the comparator is relatively easy to achieve. The comparator hysteresis can be modelled as an additive white noise at the input 


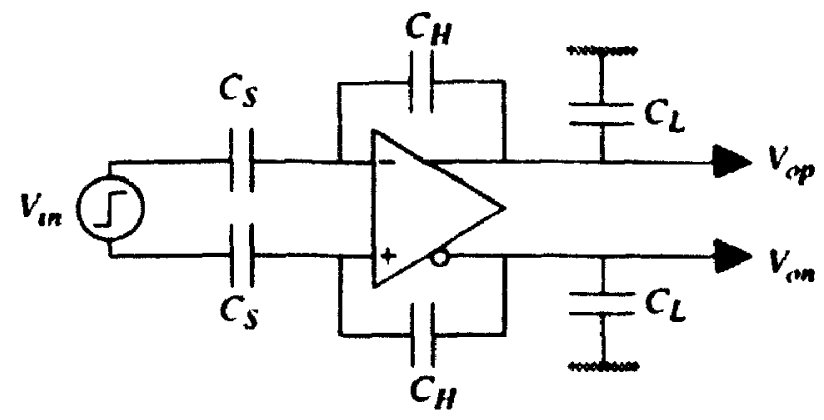

Figure 4.35: Test structure for settling time simulation

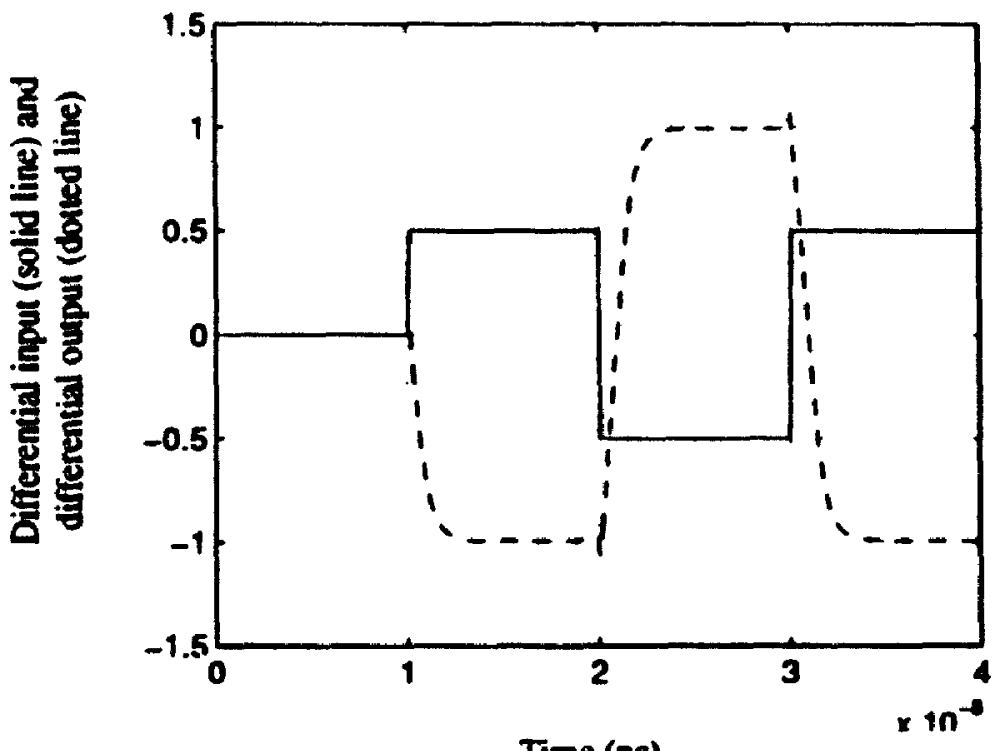

Figure 4.36: Worst case setding time simulation in Eldo.

of the comparator. Both the input referred noise and comparator hysteresis are noiseshaped (similarly to quantization noise) by the feedback loop and will be band-rejected around the center frequency $\left(f_{s} / 4\right)$. Eldo SC simulations of the fourth-order $\Sigma \Delta$ modulators show that for a hysteresis voltage of $10 \%$ of the full scale (reference levels), the in-band noise power is increased by about $1.5 \mathrm{~dB}$.

The schematic of a fully differential comparator used in the design of both modulators is shown in Figure 4.37. The first stage is a preamplifier with a gain of $22 \mathrm{~dB}$ and a unity gain bandwidth of $650 \mathrm{MHz}$. The second stage is a cross-coupled latch reset by N5. Gain and unity gain bandwidth of the second stage are $28 \mathrm{~dB}$ and $620 \mathrm{MHz}$ respectively. This 


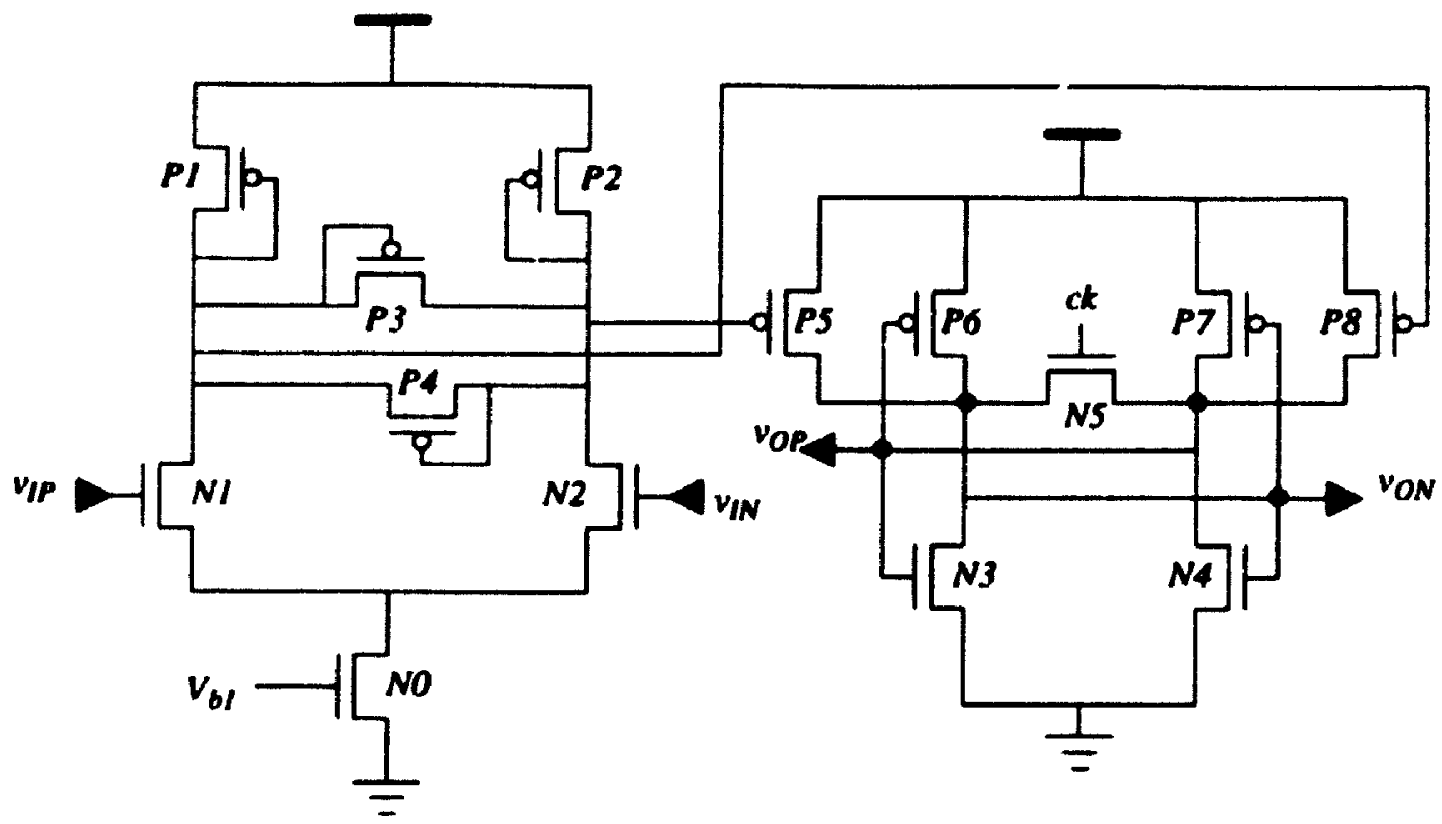

Figure 4.37: Fully differential comparator

\begin{tabular}{|c|c|}
\hline Parameter & Value \\
\hline DC gain & $54 \mathrm{~dB}$ \\
\hline Unity gain frequency & $650 \mathrm{MHz}$ \\
\hline Phase Margin & $70^{\circ}$ \\
\hline Slew rate & $1000 \mathrm{~V} / \mu \mathrm{s}$ \\
\hline Differential Output swing & $2 \mathrm{~V}$ \\
\hline $\begin{array}{c}\text { Power dissipation } \\
\text { (including CMFB circuit) }\end{array}$ & $8.8 \mathrm{~mW}$ \\
\hline
\end{tabular}

Table 4.5: Simulated characteristics of opamp for $1 \mathrm{pF}$ load capacitance comparator is followed by a cross-coupled NAND latch.

The transistor dimensions in this circuit are listed in Table 4.6.

In both fourth-order bandpass $\Sigma \Delta$ modulators, this comparator is followed by a latch. Thus, the outputs of the comparator have to drive a single logic gate with an input capacitance of $25 \mathrm{fF}$. For a $2 \mathbf{m V}$ differential input signal, the delay time (clock going 


\begin{tabular}{|c|c|c|c|c|c|c|c|c|c|c|c|c|c|c|}
\hline MOSFET & No & N1 & N2 & N3 & N4 & NS & P1 & P2 & P3 & P4 & P5 & P6 & P7 & P8 \\
\hline \hline$W(\mu \mathrm{m})$ & 200 & 200 & 200 & 4 & 4 & 8 & 50 & 50 & 50 & 50 & 10 & 1.2 & $i .2$ & 10 \\
\hline$L(\mu \mathrm{m})$ & 1 & 0.5 & 0.5 & 1 & 1 & 0.5 & 2 & 2 & 2 & 2 & 0.5 & 1 & 1 & 0.5 \\
\hline
\end{tabular}

Table 4.6: Comparator transistor sizes

low and output becoming ready) of the comparator driving $\mathbf{5 0}$ fF capacitor loads is about $2 \mathrm{~ns}$. The power consumption of the comparator at $3.3 \mathrm{~V}$ is about $3 \mathrm{~mW}$.

\subsection{Measurement Results}

This section describes measured results for the fourth-order bandpass $\Sigma \Delta$ modulator and the double-sampled fourth-order bandpass $\Sigma \Delta$ modulator. Both modulators were packaged in a 60-pin high speed metal package and were tested separately. A PCB was designed for testing the modulator. All the reference voltages, and reference currents needed for the modulators were generated on the PCB board using voltage regulators and resistors.

\subsubsection{Fourth-Order Bandpass Sizma-Delta Modulator}

The fourth-order SC bandpass $\Sigma \Delta$ modulator was tested at a clock frequency of $80 \mathrm{MHz}$ using a supply voltage of 3.3V. Measured output spectrum for this modulator for a 20.1 MHz sinusoid signal, with an amplitude of $10 \mathrm{~dB}$ below full scale, is shown in Figure 4.38.

This outpit spectrum is generated in Matlab, by performing an FFT on 65536 bits of the modulator output captured by a logic analyzer. Measured maximum SNR for this modulator is $42 \mathrm{~dB}$ in a bandwidth of $1 \mathrm{MHz}$. This value is $20 \mathrm{~dB}$ less than the expected theoretical value of $62 \mathrm{db}$.

Such a low SNR can be caused by a number of circuit imperfections, including capacitor mismatch and low opamp gain. In this process, poly-poly capacitors have good matching properties and $300 \mathrm{fF}$ unit capacitors used in this modulator are expected to match within $1 \%$. For an oversampling ratio of 40 , this capacitor mismatch reduces the SNR by only $1-2 \mathrm{~dB}$.

Unfortunately, a stand alone opamp circuit was not included in the test chip due to the 


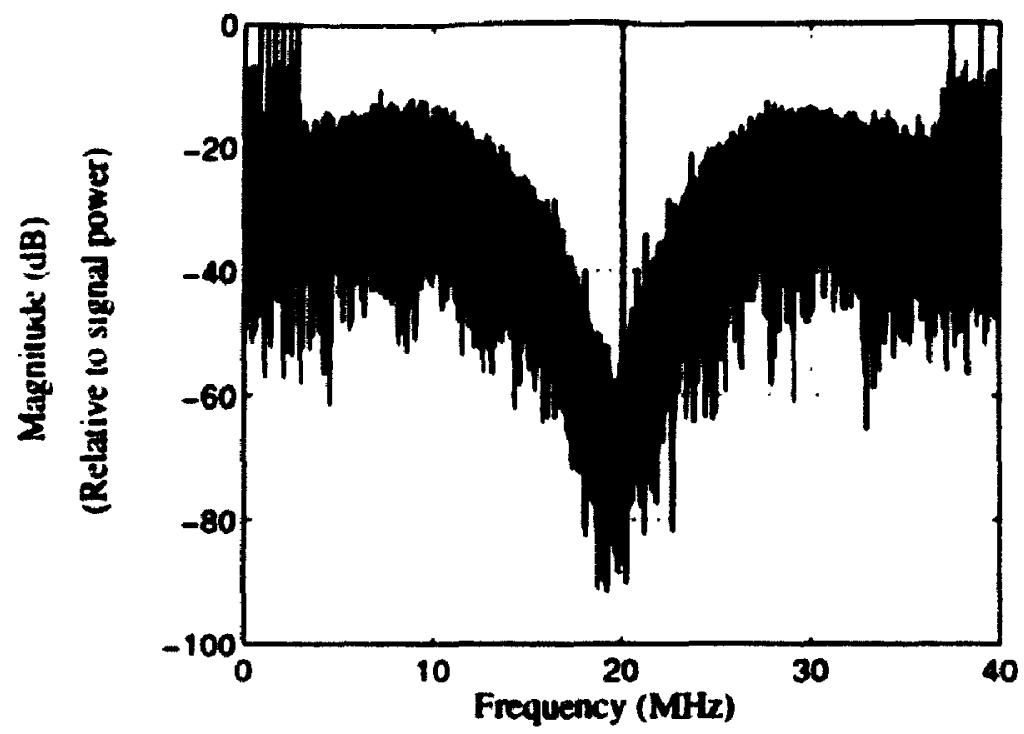

Figure 4.38: Measured output spectrum of the fourth-order bandpass sigma-delta modulator for an input signal of $10 \mathrm{~dB}$ below full scale.

limited chip area available. Therefore, opamp gain can not be measured by itself. Four indirect methods used to estimate the opamp gain consistently indicate a low DC gain of about $40-46 \mathrm{~dB}$.

First, the output conductances of a number of $0.7 \mu \mathrm{m}$ MOSFETs were measured. The measured output conductance was typically lower than the simulated value by a factor of 3 to 3.5. Since a cascode architecture is employed in this opanin, the DC gain of the amplifier is reduced by about $20 \mathrm{~dB}$ (DC gain is proportional to $r_{o}^{2}$ ). The estimated opamp DC gain is $\mathbf{4 6} \mathrm{dB}$.

Second, expanding the view of the output spectrum around the resonant frequency indicates that the notch is shifted by about $500 \mathrm{kHz}$, as shown in Figure 4.39.

In section 4.4.1, it was shown that a low opamp gain can cquse a shift in the notch frequency. For a bandwidth of $1 \mathrm{MHz}$, the shift in notch frequency is $B W / 2$, and thus according to (4-72). SNR is reduced by at least $15 \mathrm{~dB}$. Using equation (4-73), the opamp gain is estimated to be $\mathbf{4 0} \mathrm{dB}$.

Third, a low opamp gain reduces the gain of the resonator. Using equation (4-76) and $A=40 \mathrm{~dB}$, the gain of the resonator at resonance is about $H_{R 0}=6.25$. For an 


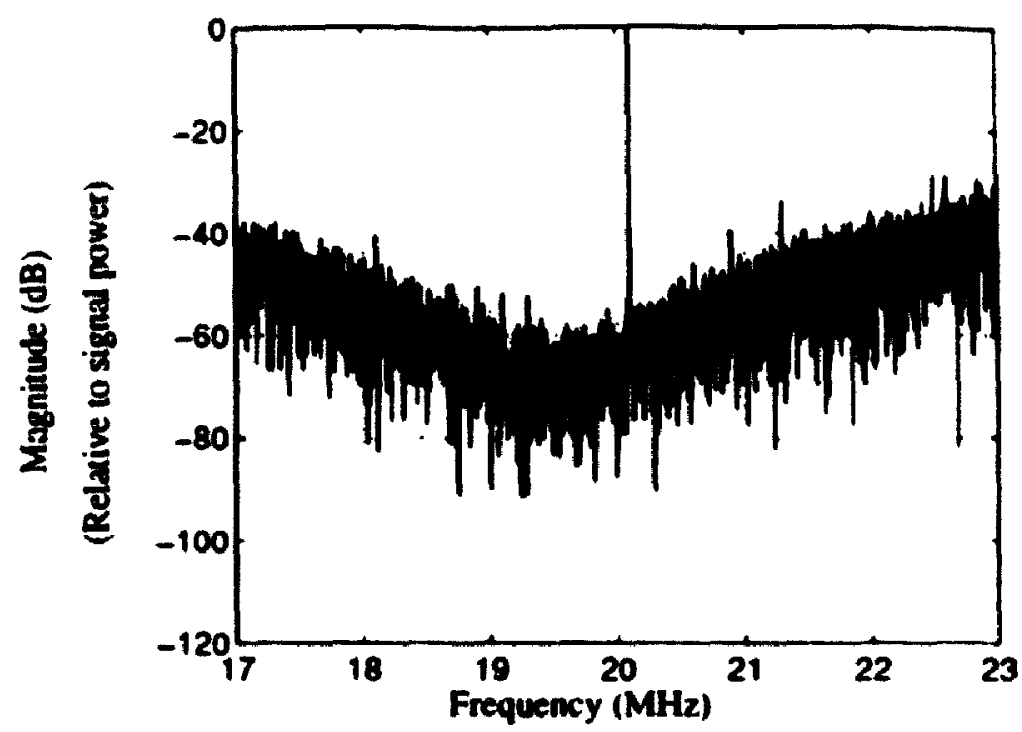

Figure 4.39: Expanded view of the output spectrum around the notch. Note that the notch frequency is shifted by about $500 \mathrm{kHz}$ to the left (lower frequency)

oversampling ratio of 40 . the ratio of the oversampling ratio to resonator gain is $(O S R) / H_{R 0}=6.4$. From Figure 4.25, in-band quantization noise increases by about $20 \mathrm{~dB}$. That is in agreement with the measured SNR of $42 \mathrm{~dB}$ which is $20 \mathrm{~dB}$ lower than the expected $62 \mathrm{~dB}$.

The fourth method of estimating the opamp gain indicates an opamp gain of 41) $\mathrm{dB}$ and is discussed in the next section.

\subsubsection{Double-Sampled Fourth-Order SC Bandpass Sigma-Detla Modulatur}

The double-sampled bandpass SC $\Sigma \Delta$ modulator was tested at a clock frequency of $80 \mathrm{MHz}$. Thus, the effective sampling rate is $160 \mathrm{MHz}$. Figure 4.40 shows the output spectrum of the modulator for an input sinusoid at $40.8 \mathrm{MHz}$ with an amplitude of $6 \mathrm{~dB}$ below full scale.

The output bit-stream was captured by a logic analyzer for 16384 clock cycles. In Matlab. a 16384-point FFT was carried out to compute the output spectrum. The image signal is at $39.2 \mathrm{MHz}$ and is $39 \mathrm{~dB}$ below the signal. This suggests that the capacitor mismatch is about $1 \%$. The SNDR of this modulator is limited to $30 \mathrm{~dB}$ for a $5 \mathrm{MHz}$ bandwidth.

Just as for the simple SC modulator, low opamp gain has shifted the notch frequency to a 


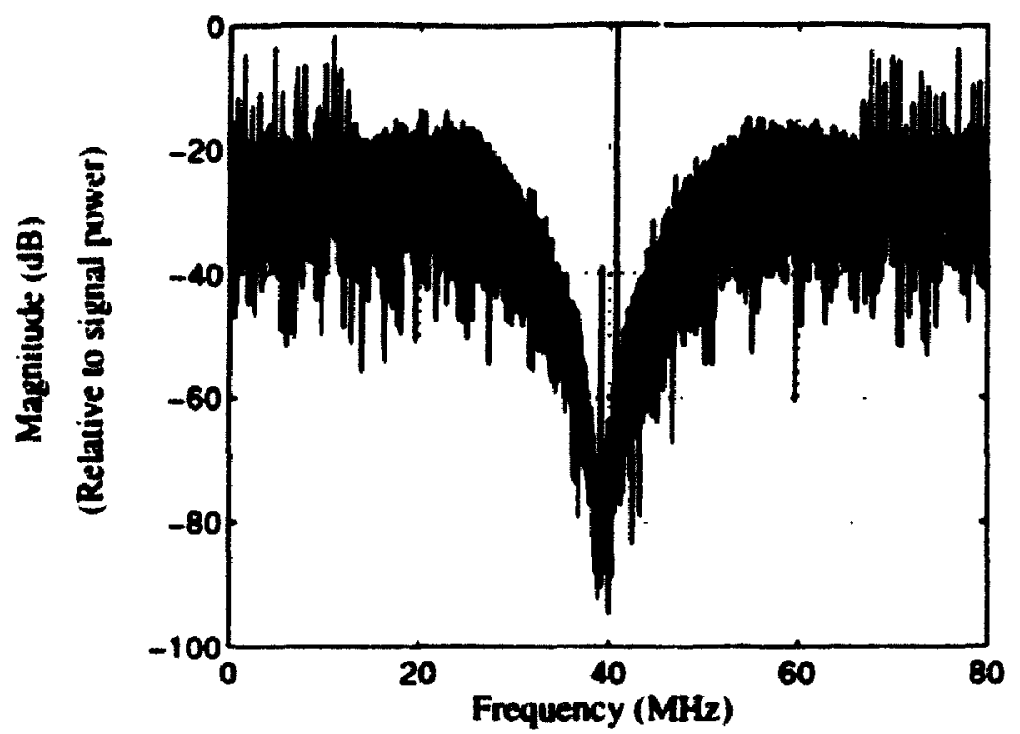

Figure 4.40: Measured output spectrum of the double-sampled fourth-order SC bandpass sigma-delta modulator for an input signal of $10 \mathrm{~dB}$ below full scale. Note that image signal is about $39 \mathrm{~dB}$ below the fundamental signal.

lower value than the expected $40 \mathrm{MHz}$. Figure 4.41 illustrates an expanded view of the output spectrum around $40 \mathrm{MHz}$.

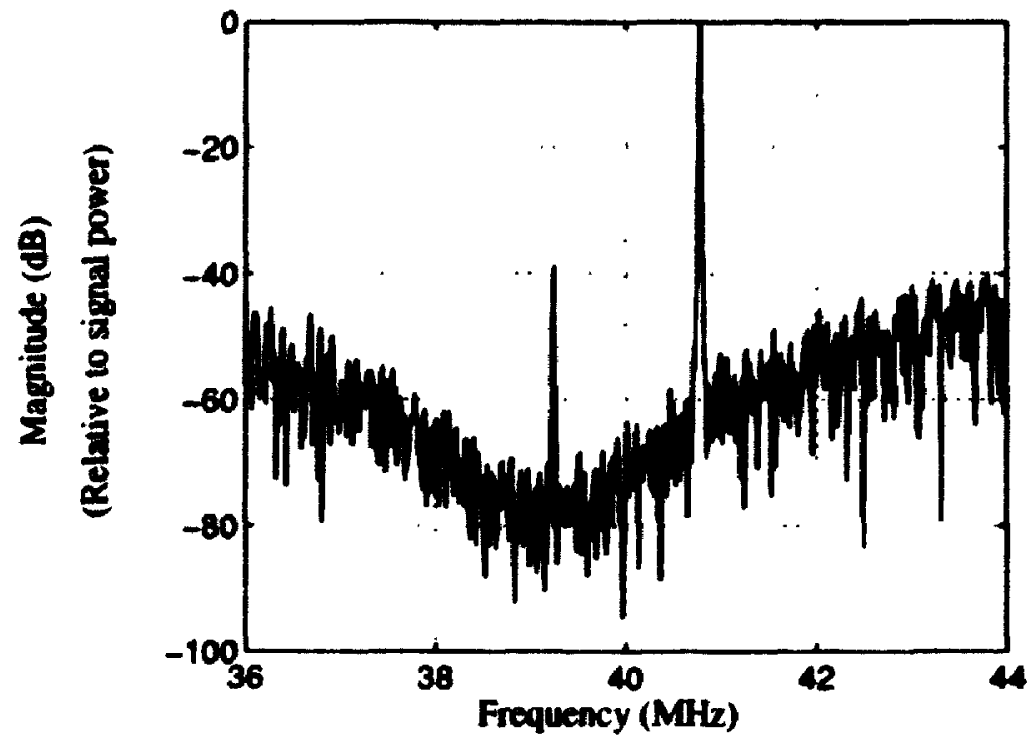

Figure 4.41: Expanded view of the output spectrum around the notch. Note that the notch frequency is shifted by about $1 \mathrm{MHz}$. 
As we can observe in Figure 4.41. the notch frequency of the modulator is shifted to about $39 \mathrm{MHz}$, which is $1 \mathrm{MHz}$ below the expected value of 4$) \mathrm{MHz}$.

Using equation (4-84), the opamp DC gain is calculated to be 4 ) $\mathrm{dB}$. This is consistent with the estimated opamp DC gain obtained in the previous section.

\subsection{Summary}

This chapter considered a z-domain architecture for a fourth-order bandpass $\mathrm{A}$ modulator. This modulator is a direct map of a second-order lowpass $\Sigma \Delta$ modulator (o) bandpass by transforming integrators to resonators.

In SC technology, an efficient method of implementing a resonator is to use two delay cells in a negative feedback loop. Half delay and full delay SC circuits were described and impacts of circuit non-idealities on the performance of these circuits were analyzed. Specifically. it was shown that tinite opamp gain causes both a gain error and a phase error in the operation of a SC delay circuit. In a SC resonator, finite opamp gain causes a change in the resonant frequency.

Two fourth-order SC bandpass $\mathrm{\Sigma A}$ modulators were presented. The first one is a singlesampled SC circuit and is shown to be faster than a previously reported one. The second is a double-sampled SC circuit. Results of Eldo simulations for these modulators were presented.

Finally, the two bandpass modulators were designed and fabricated in a $0.5 \mu \mathrm{m}$ CMOS process. Both modulators operate at a clock frequency of $80 \mathrm{MHz}$. The SNR of the single-sampled modulator is $42 \mathrm{~dB}$ in the bandwidth of $1 \mathrm{MHz}$. This is about $20 \mathrm{~dB}$ less than the expected value of $62 \mathrm{~dB}$. A low opamp DC gain appears to be responsible for the reduced SNR. Three indirect methods of estimating the opamp DC gain indicate a low opamp DC gain of $40 \mathrm{~dB}$ to $46 \mathrm{~dB}$. A SC amplifier circuit that is insensitive to low opamp gain such as [Martin87], would be a better choice for the next impt:mentation. The double-sampled modulator exhibits an image signal as low as $-40 \mathrm{~dB}$ relative to the signal. This is probably caused by capacitor mismatch in the first stage. 


\section{Chapter 5}

\section{SC Bandpass Sigma-Delta Modulato: Design In a Digital CMOS Process}

As we discussed in Chapter 2, to be cost effective it is desirable to implement analog SC circuits in standard digital CMOS processes where linear poly-to-poly capacitors are not available. In a single-poly process, a relatively low specific value linear capacitor is formed using poly and metal as electrodes and field oxide as insulator. Another $c_{\mathrm{r}}$ tion is to use MOSFET gate capacitors in the strong inversion or accumulation regime.

In this chapter, the linearity of MOSFET gate capacitors in strong inversion and accumulation is studied first. Measured CV-plots in a $0.5 \mu \mathrm{m}$ CMOS technology will show that MOSFETs biased in strong inversion are more linear than MOSFETs biased in the accumulation regime due to a gate-poly depletion effect. Distortion caused by nonlinearity of capacitors in a SC amplifier is then analyzed and some simulation results are presented. Finally, the design of a fourth-order bandpass $\Sigma \Delta$ modulator, using a pMOSFE - transistor as a linear capacitor, is described.

\subsection{MOS Capacitor}

A MOS capacitor uses polysilicon and semiconductor as the two parallel plates and silicon dioxide as insulator. In a CMOS process, gate poly and device well are used as electrodes and the thin gate oxide serves as the insulating layer. Figure 5.1a shows the structure of a MOS capacitor with p-well as the bottom plate. A MOSFFT transistor is made by adding source and drain to the sides of the MOS capacitor, as shown in Figure 5.1b. 


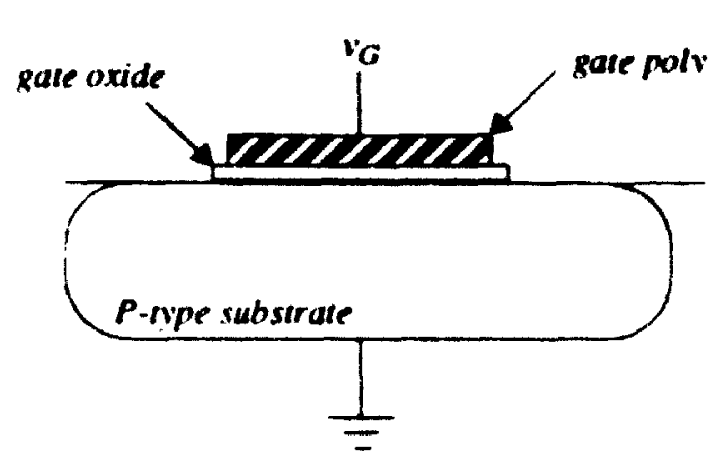

(a)

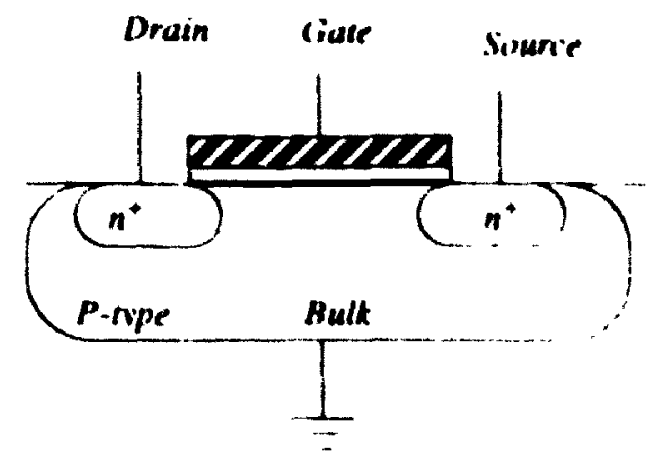

(b)

Figure 5.1: Structure of (a) a MOS capacitor and (b) an nMOSFET transistor

MOS capacitance is dependent on the gate to bulk voltage and is strongly non-linear. For a negative gate voltage $v_{G}<0$, holes will be attracted to the surface (Si-SiO $\mathrm{S}_{2}$ interfiace) of the p-type semiconductor. This mode is called accumulation and the MOS capacitor resembles a parallel plate capacitor with a capacitance per unit area of

$$
C_{G B}=C_{o x}=\frac{\varepsilon_{o x}}{t_{o x}}
$$

where $\varepsilon_{o x}$ is the oxide dielectric constant and $t_{o x}$ is the oxide thickness. When the applied gate voltage is $0<v_{G}<V_{i}$. the holes are repelled from the surface and a negative depletion layer is formed near the surface. In this regime. called depletion. the MOS capacitor has two dielectrics (the gate oxide and the depleted silicon) in series. Thus, the MOS capacitance in depletion mode is given by the series combination of the gate capacitance $\left(C_{o x}\right)$ and the depletion capacitance.

$$
C_{G B}=\left(\frac{t_{o x}}{\varepsilon_{o x}}+\frac{d}{\varepsilon_{s i}}\right)^{-1} .
$$

Here, $\varepsilon_{s i}$ is the semiconductor dielectric constant and $d$ is the depletion layer width. As gate voltage is increased, the depletion layer width increases and hence the gate to bulk capacitance decreases.

If the gate voltage is increased beyond the threshold voltage $v_{G}>V_{i}$, the concentration of attratted electrons at the surface will be greater than the concentration of the holes at the 
surface. This condition is called strong inversion. The inversion layer below the oxide provides the bottom plate of the capacitor, and the gate to bulk capacitance is given by the oxide capacitance $\left(C_{o x}\right)$. In the two-terminal MOS capacitor, the inversion layer is formed by the thermal generation of carriers, which is a slow process. In a MOSFET capacitor, the source terminal will provide carriers for the formation of the inversion layer. Thus, the characteristic of a MOSFET capacitor at high frequency will be the same as the low frequency $C-V$ characteristic of the two-terminal MOS capacitor.

The theoretical C-V characteristic of an nMOSFET, using analytical results in [Tsividis87], is shown in Figure 5.2.

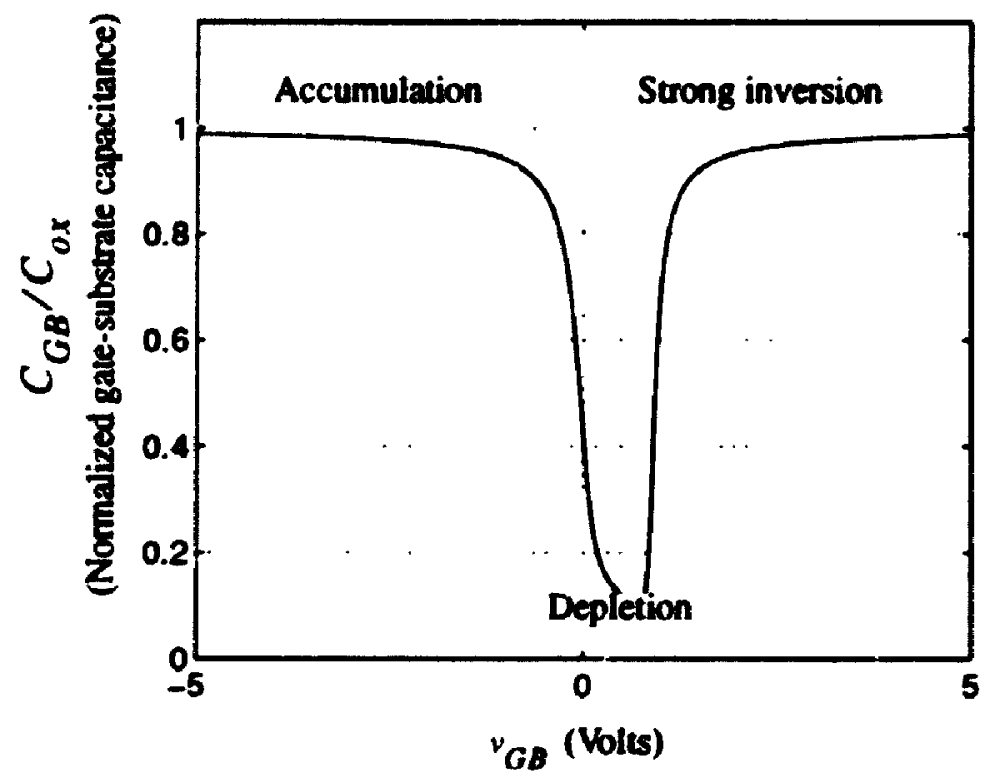

Figure 5.2: Analytical C-V characteristics of an nMOSFET transistor

As we can observe in the C-V plot, Figure 5.2, the MOSFET capacitance is relatively linear (or weakly non-linear) in the accumulation and strong inversion regimes.

The capacitance of a voltage-dependent non-linear capacitor can be expressed in Taylor series form by

$$
C(v)=C_{0}\left(1+\alpha_{1} v+\alpha_{2} v^{2}+\ldots\right)
$$

Typically, voltage coefficien.: of the cubic and higher terms are small and the capacitance can be approximated by the first three terms in equation (5-3). 
The voltage coefficients of a MOSFET capacitance in strong inversion are given by [Behr92]

$$
\alpha_{1}=-\frac{2 \phi_{1}}{\left|V_{R}-V_{1}\right|^{2}}
$$

and

$$
\alpha_{2}=\frac{2 \phi_{t}}{\left|V_{R}-V_{d}\right|^{3}}
$$

Here. $\phi_{t}$ is the thermal voltage and $V_{R}$ is the bias voltage. In accumulation. the voltage coefficients are given by similar expressions with $V_{t}$ being substituted with the flat-band voltage $\left(V_{F B}\right)$. Therefore, the non-linearity of the MOSFET capacitor in strong inversion and accumulation regions are theoretically almost identical.

In the above analysis, it is assumed that the poly gate is degenerately doped. In some advanced processes, the poly gate is doped along with the source and drain diffusion. In this case, the gate is not doped degenerately. A positive voltage on the gate electrode will pull the electrons in the polysilicon to the top, and the bottom of the poly gate will be depleted. Therefore, the effective dielectric thickness of the capacitor will increase. This phenomenon, known as "poly depletion," will cause a slight roll-off in the C-V curve in the strong inversion region near the supply voltage.

Figure 5.3 illustrates C-V characteristics for an nMOSFET transistor in a $0.5 \mu \mathrm{m}$ CMOS technology obtained from silicon measurement (solid line) and simulation (dashed line) using the MISNAN model. Note the negative slope in strong inversion of the measured (solid line) C-V curves. As we can observe in this figure, poly-depletion is not modelled by MISNAN and linearity of a MOSFET in the accumulation regime is optimistically predicted to be high. Similarly, linearity of a MOSFET in the strong inversion regime is predicted pessimistically to be low by the MISNAN model. Gate-poly depletion appears to largely compensate the non-linearity of MOSFET capacitors in the strong inversion regime.

The C-V characteristic of pMOSFET is shown in Figure 5.4. 


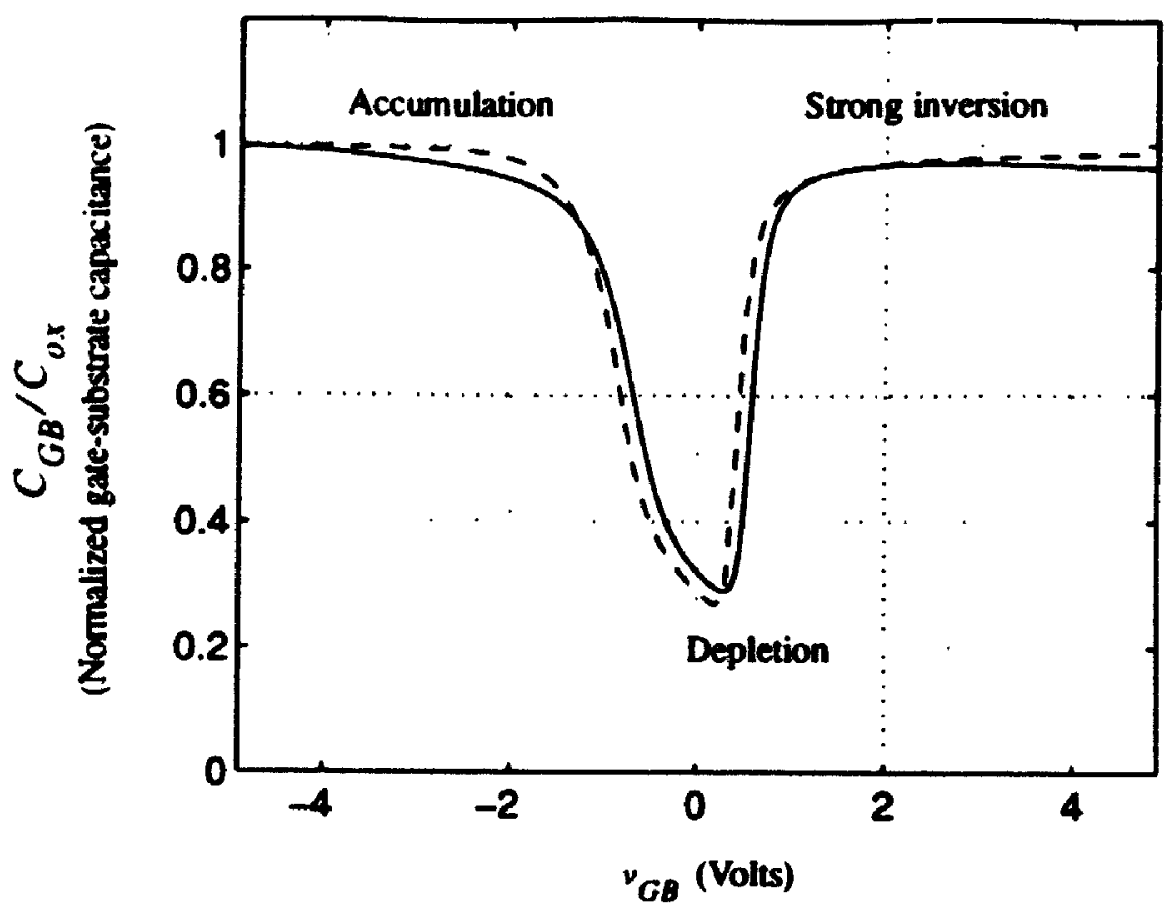

Figure 5.3: Measured (solid line) and simulated (dashed line) C-V plot of an nMOSFET

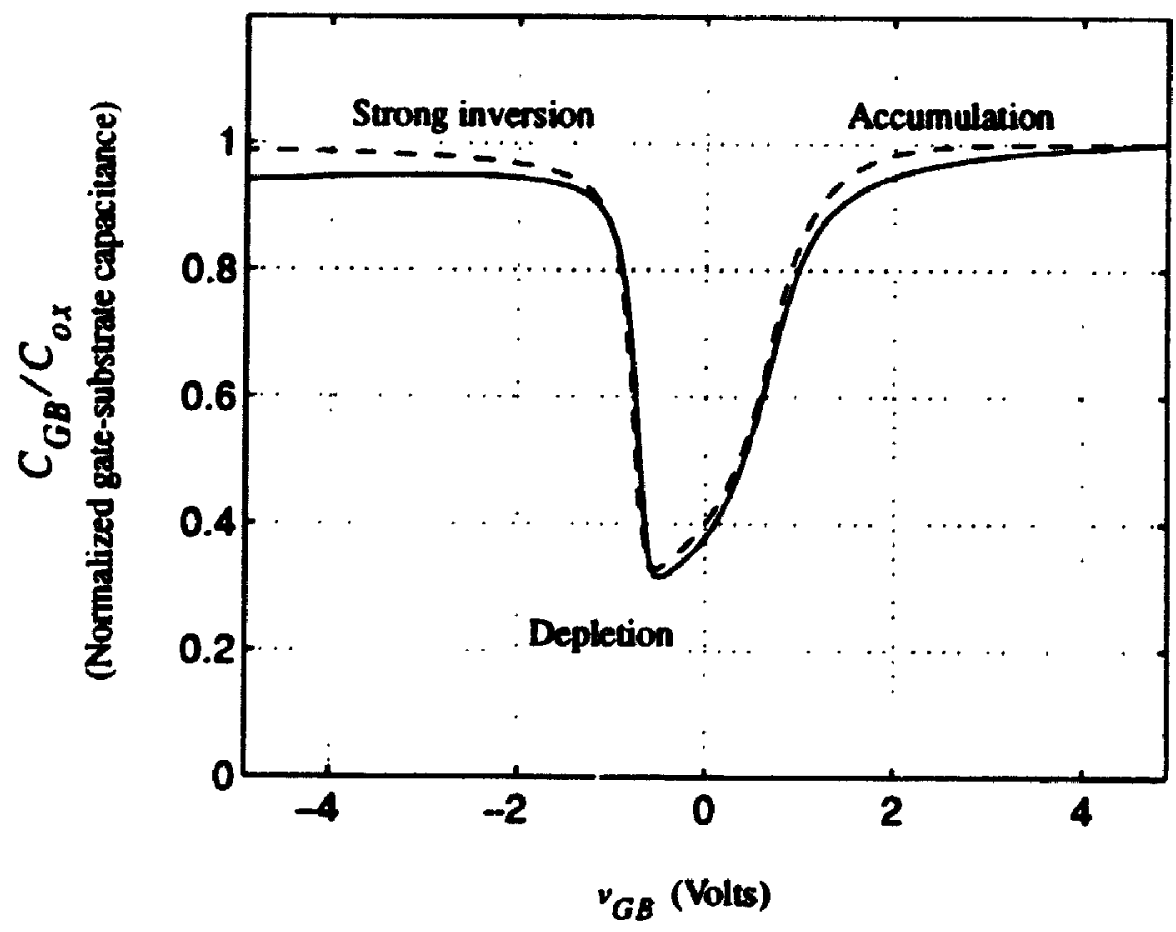

Figure 5.4: Measured (solid line) and simulated (dashed line) C-V plot of a pMOSFET 
Both nMOSFET and pMOSFET are strongly non-linear in the $-2 \mathrm{~V}<v_{G B}<2 \mathrm{~V}$ range and only weakly non-linear when MOSFETs are biased by more than $2 \mathrm{~V}$ in either accumulation or strong inversion regimes. From the measured $\mathrm{C}-\mathrm{V}$ plots, we can see that both MOSFETs exhibit a better linearity in the strong inversion regime than in the accumulation regime. This has been verified in Matlab and will be discussed below.

Using the polyfit feature in Matlab (least squares algorithm), the first $\left(\alpha_{1}\right)$ and second $\left(\alpha_{2}\right)$ order voltage coefficients of measured MOSFET capacitance are found for different ranges of $v_{G S}$. Table 5.1 and Table 5.2 summarize the results for the nMOSFET and pMOSFET transistors respectively.

\begin{tabular}{|c|c|c|c|c|}
\hline \multirow{2}{*}{ Condition } & \multicolumn{2}{|c|}{ Measured } & \multicolumn{2}{c|}{ Simulated } \\
\cline { 2 - 5 } & $\alpha_{1}\left(v^{-1}\right)$ & $\alpha_{2}\left(v^{-2}\right)$ & $\alpha_{1}\left(v^{-1}\right)$ & $\alpha_{2}\left(v^{-2}\right)$ \\
\hline \hline Strong Inversion $\left(2 \mathrm{~V}<v_{G S}<3 \mathrm{~V}\right)$ & 0.0279 & 0.0050 & 0.0327 & 0.0045 \\
\hline Accumulation $\left(-3 \mathrm{~V}<v_{G S}<-2 \mathrm{~V}\right)$ & 0.125 & 0.0177 & 0.1457 & 0.0251 \\
\hline Strong Inversion $\left(3 \mathrm{~V}<v_{G S}<4 \mathrm{~V}\right)$ & 0.005 & 0.0012 & 0.0162 & 0.0016 \\
\hline Accumulation $\left(-4 \mathrm{~V}<v_{G S}<-3 \mathrm{~V}\right)$ & 0.045 & 0.0049 & 0.0114 & 0.0014 \\
\hline
\end{tabular}

Table 5.1: nMOSFET capacitance voltage coefficients

\begin{tabular}{|c|c|c|c|c|}
\hline \multirow{2}{*}{ Condition } & \multicolumn{2}{|c|}{ Measured } & \multicolumn{2}{c|}{ Simulated } \\
\cline { 2 - 5 } & $\alpha_{1}\left(v^{-1}\right)$ & $\alpha_{2}\left(v^{-2}\right)$ & $\alpha_{1}\left(v^{-1}\right)$ & $\alpha_{2}\left(v^{-2}\right)$ \\
\hline \hline Strong Inversion $\left(-3 \mathrm{~V}<v_{G S}<-2 \mathrm{~V}\right)$ & 0.0427 & 0.0077 & 0.0534 & 0.0079 \\
\hline Accumulation $\left(2 \mathrm{~V}<v_{G S}<3 \mathrm{~V}\right)$ & 0.1264 & 0.0180 & 0.1079 & 0.0182 \\
\hline Strong Inversion $\left(-4 \mathrm{~V}<v_{G S}<-3 \mathrm{~V}\right)$ & 0.0162 & 0.0027 & 0.0499 & 0.0064 \\
\hline Accumulation $\left(3 \mathrm{~V}<v_{G S}<4 \mathrm{~V}\right)$ & 0.0403 & 0.0036 & 0.0134 & 0.0017 \\
\hline
\end{tabular}

Table 5.2: pMOSFET capacitance voltage coefficients

For similar gate to substrate voltages, MOSFETs biased in strong inversion consistently have a significantly better linearity than MOSFETs operating in accumulation regime.

For low-voltage linear SC circuits, it is desirable to extend the linear portions of the C-V 
curve closer to the $v_{G B}=0$ axis, so that less bias voltage will be required to operate the MOSFET in the strong inversion (or accumulation) regime. Low- $V$, natural MOSFETs are suited very well for this purpose. Due to their low threshold voltage, natural MOSFETs have a wider linear range in the strong inversion regime. Figure 5.5 shows the C.V plots for both a natural MOSFET and a threshold adjusted MOSFET transistor.

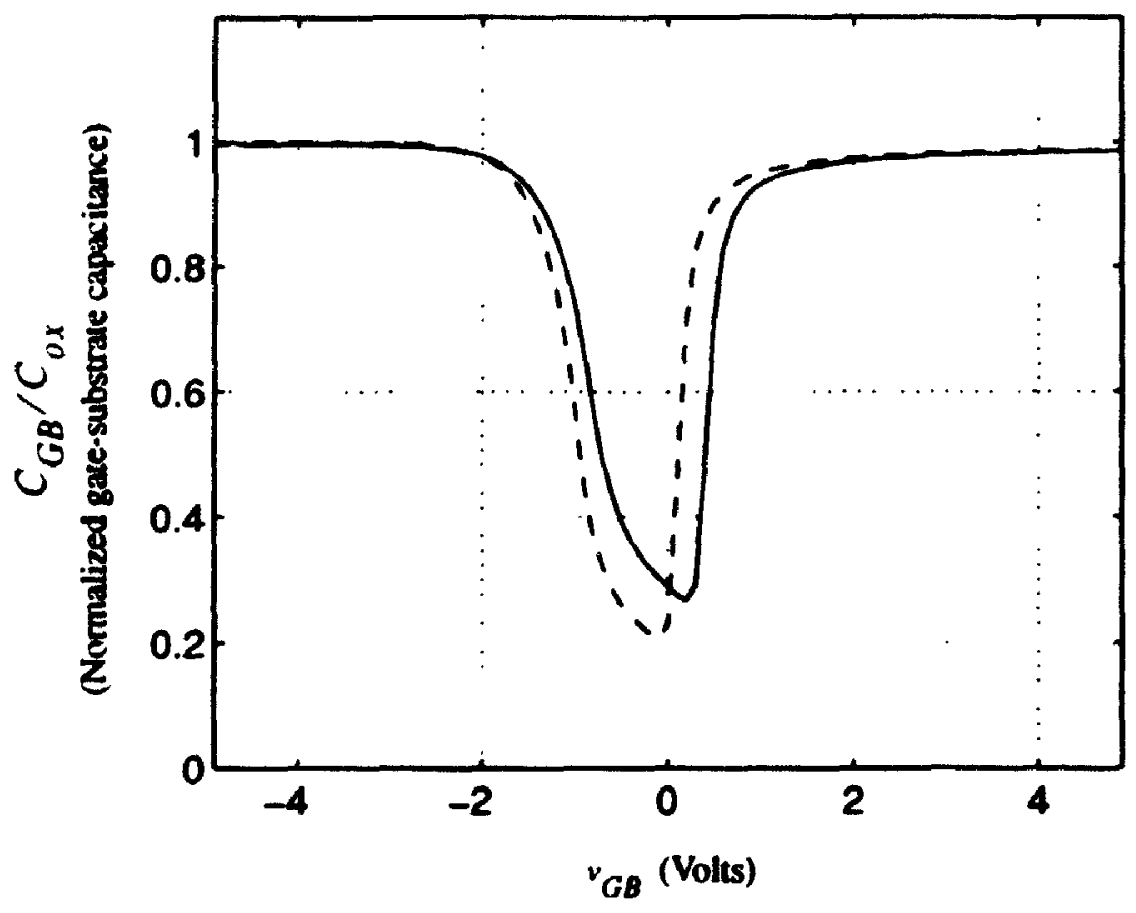

Figure 5.5: Simulated C-V characteristics of a low- $V_{t}$ natural nMOSFET (dashed line) and a threshold adjusted nMOSFET (solid line)

Note that the linear range of natural nMOSFET capacitance is increased (compared to that of the threshold adjusted nMOSFET) in the strong inversion region at the expense of a reduced linear range in accumulation.

\subsection{Distortion in SC Amplifier Caused by Non-linear Capacitors}

Non-linear capacitors cause distortion in SC circuits. Consider a single-ended SC amplitier as shown in Figure 5.6. Capacitors are assumed to have a similar non-linearity and their capacitances are approximated by

$$
C_{s}=C_{S 0}\left(1+\alpha_{1} v+\alpha_{2} v^{2}\right)
$$


and

$$
C_{H}=C_{H 0}\left(1+\alpha_{1} v+\alpha_{2} v^{2}\right)
$$

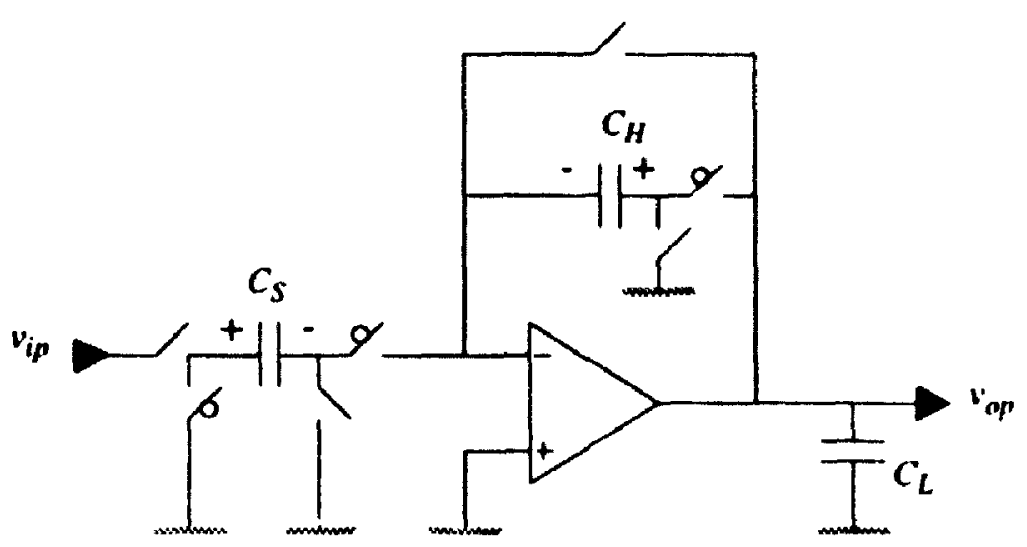

Figure 5.6: A single-ended SC amplifier

Assuming an ideal opamp, charge conservation on the sarnpling capacitor $\left(C_{S}\right)$ and the holding capacitor $\left(C_{H}\right)$ during $\phi_{1}$ and $\phi_{2}$ yields

$$
v_{o p}\left(n+\frac{1}{2}\right)=\frac{C_{S}}{C_{H}} \cdot v_{i p}(n)
$$

If the capacitors are linear, the output of the SC amplifier is a scaled version of the input signal delayed by a half-clock period.

For non-linear capacitors, substituting (5-6) and (5-7) into (5-8), the abeve difference equation becomes

$$
v_{o p}\left(n+\frac{1}{2}\right)=\frac{C_{S 0}}{C_{H 0}} \cdot \frac{1+\alpha_{1} v_{i p}(n)+\alpha_{2} v_{i p}^{2}(n)}{1+\alpha_{1} v_{o p}(n+1 / 2)+\alpha_{2} v_{o p}^{2}(n+1 / 2)} \cdot v_{i p}(n)
$$

For $\alpha_{1} \ll 1$ and $\alpha_{2} * 1$, the input-output difference equation can be approximated by

$$
v_{o p}\left(n+\frac{1}{2}\right)=\frac{C_{S 0}}{C_{H 0}}\left\{v_{i p}(n)+\alpha_{1}\left[v_{i p}(n)-v_{o p}\left(n+\frac{1}{2}\right)\right] v_{i p}(n)+\alpha_{2}\left[v_{i p}^{2}(n)-v_{o p}^{2}\left(n+\frac{1}{2}\right)\right] v_{i p}(n)\right) \cdot(5-10)
$$

The above transfer characteristic indicates that a non-linear capacitor causes non-linear 
errors at the output of the SC amplifier.

Let us assume that the input signal is a sampled sinusoid given by

$$
v_{i p}(n)=V_{i p} \cos (n \omega T)
$$

If the non-linear coefficients are small, the output of the amplifier in the error terms of equation (5-10) can be approximated to the first order by the output of an ideal SC amplitier,

$$
v_{o p}\left(n+\frac{1}{2}\right)=\frac{C_{S 0}}{C_{H 0}} \cdot v_{i p} \cos (n \omega T) .
$$

Substituting (5-11) and (5-12) into (5-10) and using the following trigonometric identities

$$
(\cos A)^{2}=\frac{1+\cos 2 A}{2} \quad \text { and } \quad(\cos A)^{3}=\frac{3 \cos A+\cos 3 A}{4}
$$

equation (5-10) becomes

$$
\begin{aligned}
v_{o p}\left(n+\frac{1}{2}\right)=\frac{c_{s 0}}{c_{H 0}} \cdot\left\{\frac{\alpha_{1}}{2}\left(1-\frac{c_{s 0}}{c_{H 0}}\right) v_{i p}+\left[1+\frac{3 \alpha_{2}}{4}\left(1-\frac{c_{s 0}^{2}}{c_{H 0}^{2}}\right) v_{i p}^{2}\right] v_{i p} \cos (n \omega T)\right. \\
+\frac{\alpha_{1}}{2}\left(1-\frac{c_{s 0}}{c_{H 0}}\right) v_{i p}^{2} \cos (2 n \omega T)+\frac{\alpha_{2}}{4}\left(1-\frac{c_{s 0}^{2}}{c_{H 0}^{2}}\right) v_{i p}^{3} \cos (3 n \omega n\}
\end{aligned}
$$

The second term in the brace brackets on the RHS of the above equation is the output at the fundamental frequency. The first term is an offset, the third and fourth terms are the error terms containing the second and third harmonics of the signal. Using the following definition for $\boldsymbol{k}$-th harmonics,

$$
H D_{k}=\frac{\text { Signal Amplitude at }(k \omega)}{\text { Signal Amplitude at Fundamental }(\omega)}
$$

the second and third hamonics for the SC amplifier circuit are found to be approximately

$$
H D_{2} \approx \frac{\alpha_{1}}{2}\left(1-\frac{C_{S O}}{C_{H 0}}\right) V_{i p}
$$

and 


$$
H D_{3}=\frac{\alpha_{2}}{12}\left[1-\left(\frac{C_{S 0}}{C_{H 0}}\right)^{2}\right] v_{i p}^{2}
$$

In a fully differential circuit, the second harmonic component is rejected at the output.

Example 5.1: In the SC amplitier of Figure 5.6, the gain of the circuit is 0.5. opamp and switches are ideal, and capacitors are poly-poly capacitors with voltage coefticients of $\alpha_{1}=10 \mathrm{ppm} / \mathrm{V}$ and $\alpha_{2}=10 \mathrm{ppm} / \mathrm{V}^{2}$. For a $2 \mathrm{~V}$ peak-to-peak input sinusuid. the second and the third harmonics are calculated from (5-16) and (5-17) respectively to be $H D_{2}=-102.5 \mathrm{~dB}$ and $H D_{3}=-110 \mathrm{~dB}$. If capacitors are implemented with pMOSFETs in strong inversion with voltage coefficients of $\alpha_{1}=42 \mathrm{kppm} / \mathrm{V}$ and $\alpha_{2}=7.7 \mathrm{kppm} / \mathrm{V}^{2}$, the distortions will be $H D_{2}=-45.6 \mathrm{~dB}$ and $H D_{3}=-78.4 \mathrm{~dB}$ for a 1 Vpp input signal.

\subsection{A SC Delay Circuit Using pMOSFET Capacitors}

In section 5.1, it was shown that pMOSFETs biased in strong inversion $\left(v_{(i B}<\left|V_{t p}\right|\right)$ exhibit a weakly non-linear behavior over a small gate-substrate voltage range. For instance, measured $\mathrm{C}-\mathrm{V}$ characteristics show that for a $1 \mathrm{~V}$ signal swing in the range of $-3 \mathrm{~V}<v_{G B}<-2 \mathrm{~V}$, the voltage coefficients of the MOSFET capacitor are $\alpha_{1}=42 \mathrm{kppm} / \mathrm{V}$ and $\alpha_{2}=7.7 \mathrm{kppm} / \mathrm{V}^{2}$. The schematic of a fully differential $\mathrm{SC}$ gain stage with unity gain during $\phi_{1}$ and a gain of half during $\phi_{2}$, using pMOSFET capacitors, is shown in Figure 5.7. The differential structure cancels even-order harmonics, reducing the importance of $\alpha_{1}$.

Transistors $M 1$ and $M 3$ are equal size pMOSFETs and $M 2$ is made of two pMOSFETs identical to $M 1$. All pMOSFET capacitors are biased to operate in strong inversion by means of different input and output common mode voltages. The gates of the pMOSFETs are connected to the input common-mode level and the bulk terminals (which is shorted to the drain and the source) are connected to the (higher) output common-mode level. For a signal swing of $v_{p p}$ with respect to $V_{o c m}$, the operating gate-to-substrate of the pMOSFET voltage is 


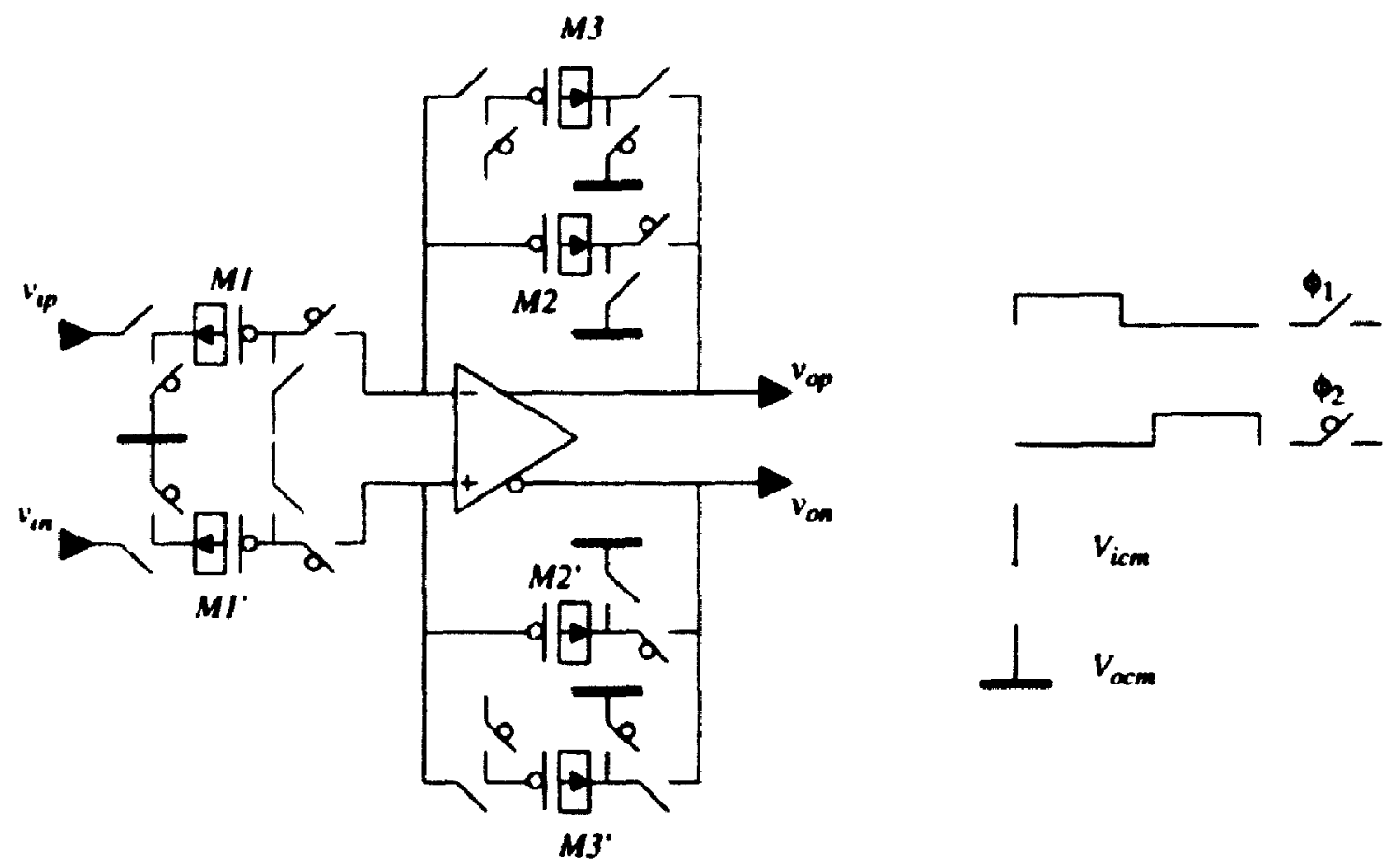

Figure 5.7: SC delay cell using pMOSFET capacitors

$$
v_{i c m}-\left(v_{o c m}+\frac{v_{p p}}{2}\right)<v_{G B}<V_{i c m}-\left(V_{o c m}-\frac{v_{p p}}{2}\right)
$$

If the input common mode voltage is set to $\mathrm{IV}$ and the output common-mode is set to $3.5 \mathrm{~V}$, then with a $1 \mathrm{~V}$ peak-to-peak signal the pMOSFET will operate in the strong inversion regime with a gate-to-bulk voltage of $-3 \mathrm{~V}<v_{G B}<-2 \mathrm{~V}$.

For a I Vpp sinusoid input signal, the output signal will be $0.5 \mathrm{Vpp}$ during $\phi_{2}$, with a delay of a half-clock period. Due to the weak non-linearity of the pMOSFETs in strong inversion, a third harmonic is also generated. The voltage coefficient $\alpha_{2}$ that is responsible for the third harmonics was calculated (Table 5.1) to be $7.7 \mathrm{kppm} / \mathrm{V}$ in the operating regime of $-3 \mathrm{~V}<v_{G B}<-2 \mathrm{~V}$. The third harmonic distortion is calculated from (5-17) to be $-78.4 \mathrm{~dB}$.

This circuit was simulated in Eldo using an ideal opamp with a gain of $100 \mathrm{~dB}$, ideal switches with an on-resistance of $1 \Omega$, and a $W / L=30 \mu \mathrm{m} / 10 \mu \mathrm{m}$ pMOSFET as urit capacitor. The non-overlapping clock had a frequency of $100 \mathrm{MHz}$ and the input signal was a I Vpp sinusoid with a frequency of $9.99 \mathrm{MHz}$. The output spectrum, Figure 
5.8. has a tone at the input frequency (its fundamental frequency) and a third harmonic whose power is $80 \mathrm{~dB}$ below the output power at the fundamental frequency.
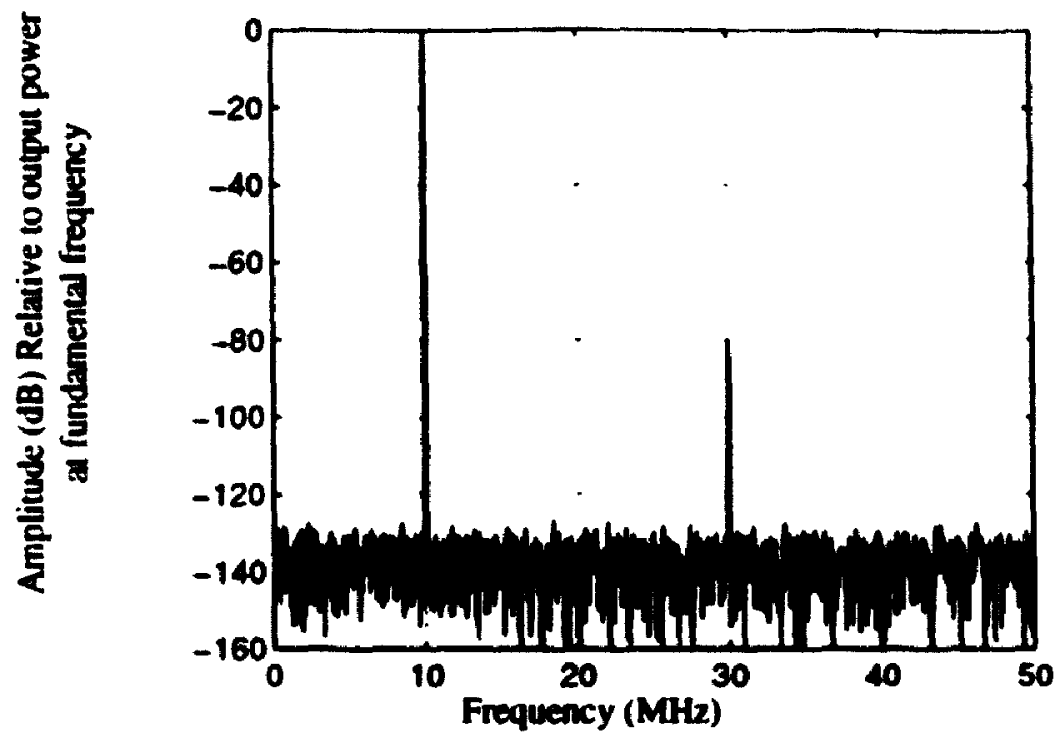

Figure 5.8: Output spectrum of the fully differential SC gain stage, Figure 5.7, during $\phi_{2}$.

As we can see in Figure 5.8, no second harmonic is generated at the output due to fully differential implementation of this circuit. In a real implementation, mismatches and offsets would cause a non-zero $\mathrm{HD}_{2}$.

During $\phi_{1}$, the output is ideally an exact replica of the input delayed by a full clock period. Since the gate to source voltage of the pMOSFET capacitors $M 1$ and $M 3$ are the same, no distortion should appear at the output. This argument is veritied by simulation and Figure 5.9 illustrates the output spectrum during $\phi_{1}$.

\subsection{A Fourth-Order SC Bandpass Sigma-Delta Modulator Using pMOSFET Capacitors}

The fourth-order bandpass $\Sigma \Delta$ of Figure 4.21 can be implemented in a standard digital CMOS process by replacing all the poly-poly capacitors with pMOSFET capacitors. The modified schematic is shown in Figure 5.10, where pMOSFET capacitors are biased in strong inversion. In this circuit all the pMOSFET capacitors are identical with a gate area of $W L$, except for the two marked by asterisk which have a gate area of $2 W L$. 


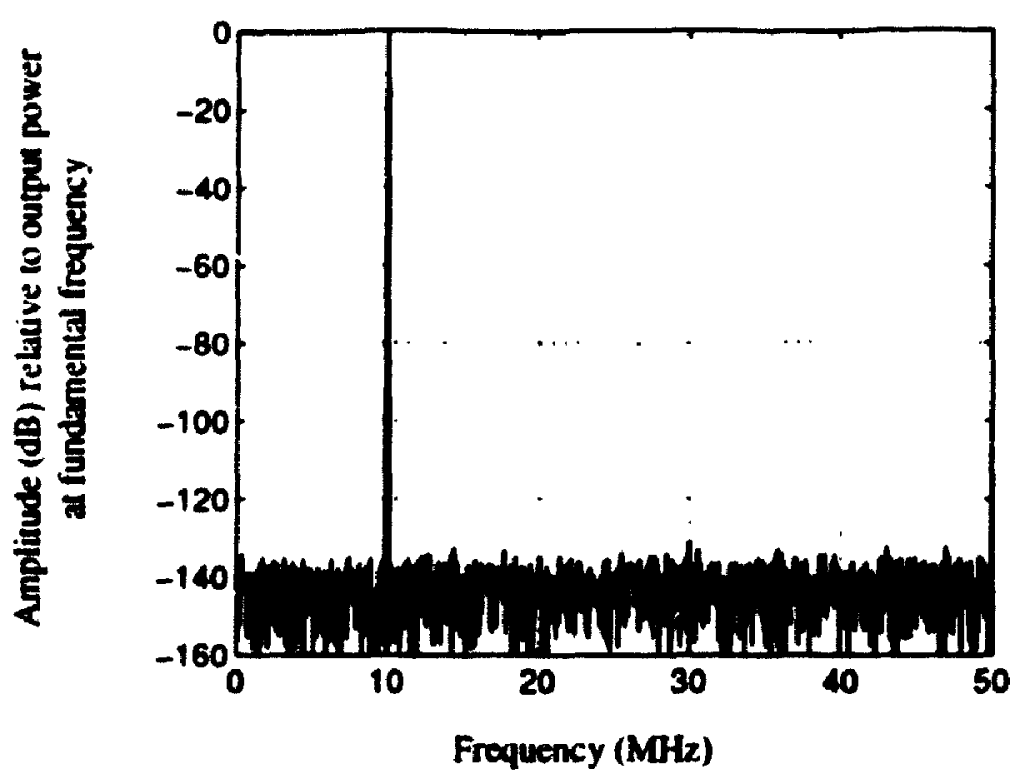

Figure 5.9: Output spectrum of the fully differential SC unity gain stage, Figure 5.7, during $\phi_{1}$

As was mentioned in the previous section, a unity gain operation (not involving summation) does not produce any harmonics. Thus, operations involving distortion include transitions from $\phi_{1}$ to $\phi_{2}$ in the first and second delay cells, as well as transition from $\phi_{2}$ to $\phi_{1}$ in the third delay cell. The fourth delay cell has a unity gain (in both phases) and is not used in a summing configuration, and therefore is free from any distortions.

This circuit was simulated in the Eldo analog simulator using nearly ideal opamps (DC gain of $60 \mathrm{~dB}$ ), ideal switches ( $\Omega$ on-resistance), and the pessimistic MISNAN model for pMOSFET capacitors. All pMOSFET capacitors are biased $2.5 \mathrm{~V}$ into in surong inversion by selecting input and output common mode levels to be at $1 \mathrm{~V}$ and $3.5 \mathrm{~V}$ respectively. Figure 5.11 shows the output spectrum of the modulator for an input sinusoid of $6 \mathrm{~dB}$ below full scale amplitude and a frequency of $24.9 \mathrm{MHz}$. The non-overlapping clock frequency was set to $100 \mathrm{MHz}$. Simulated SNR is $97 \mathrm{dR}$ and $63 \mathrm{~dB}$ for bandwidths of $200 \mathrm{kHz}$ and $1 \mathrm{MHz}$ respectively.

\section{Design Considerations}

In order to properly bias pMOSFET capacitors in the strong inversion regime, an opamp 


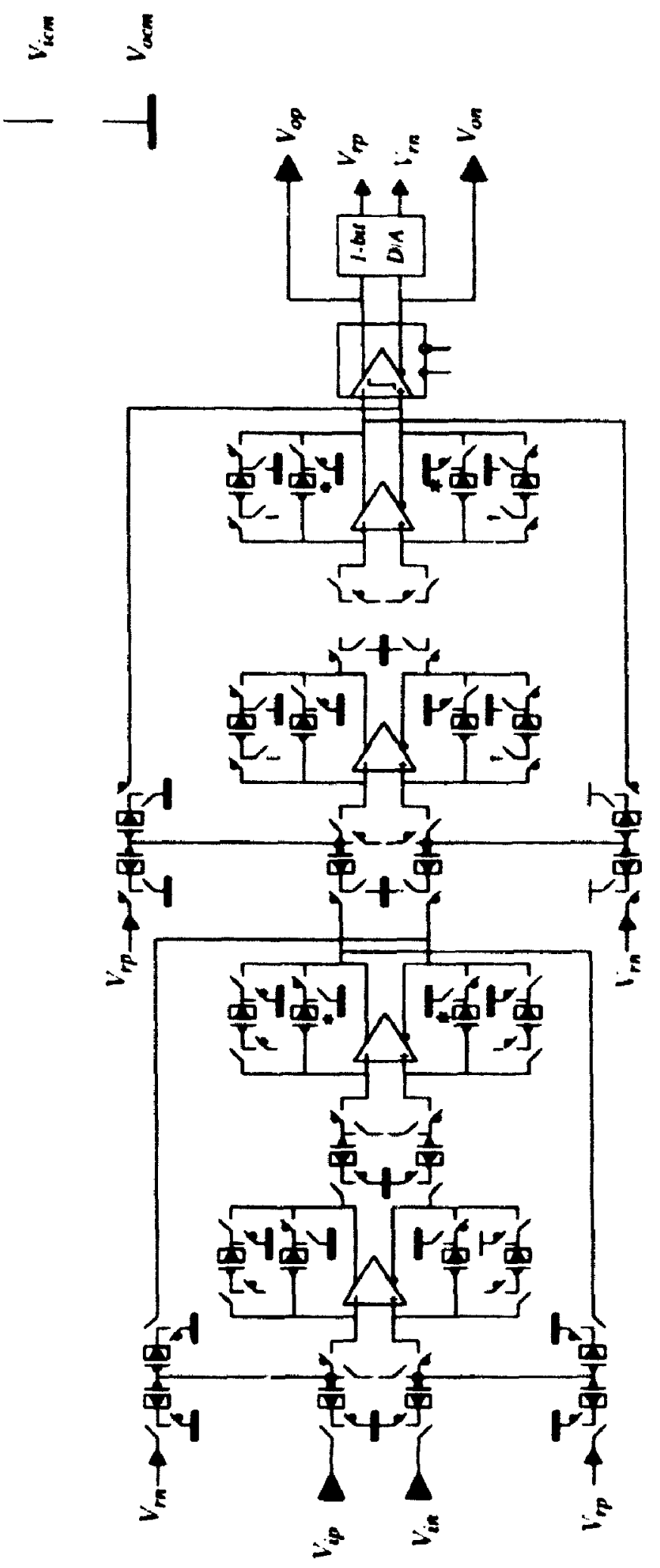

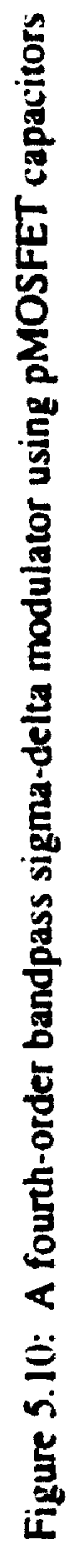




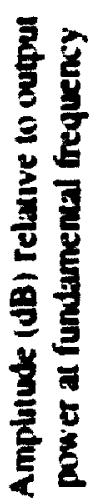

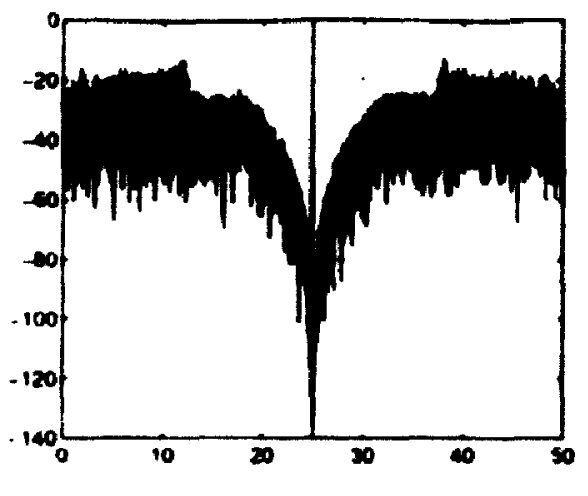

Frequency (MHz)
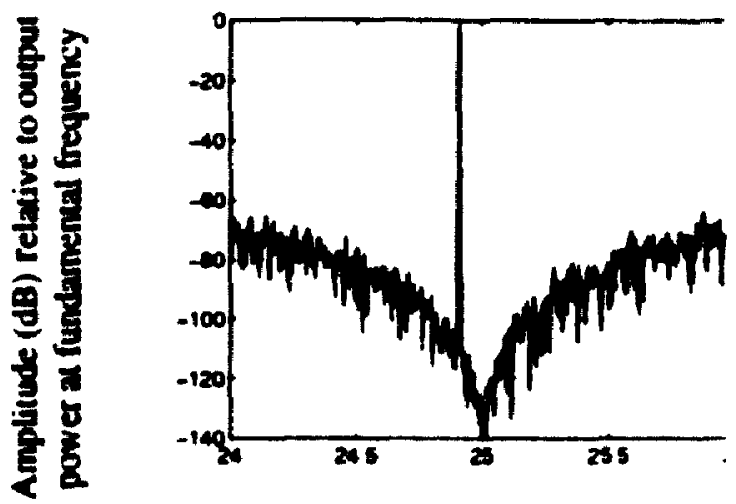

Frequency $(\mathbf{M H z})$

Figure 5.11: Output spectrum of the fourth-order SC bandpass sigma-delta modulator of Figure 5.10 for an input signal of $6 \mathrm{~dB}$ below full scale

with different input and output common mode levels is required. An efficient opamp architecture having different input and output common mode voltages is a non-folded cascode opamp, as discussed in section 4.6. The schematic of the opamp (not including the CMFB circuit) is repeated in Figure 5.12. The input common-mode level is $1 \mathrm{~V}$ and

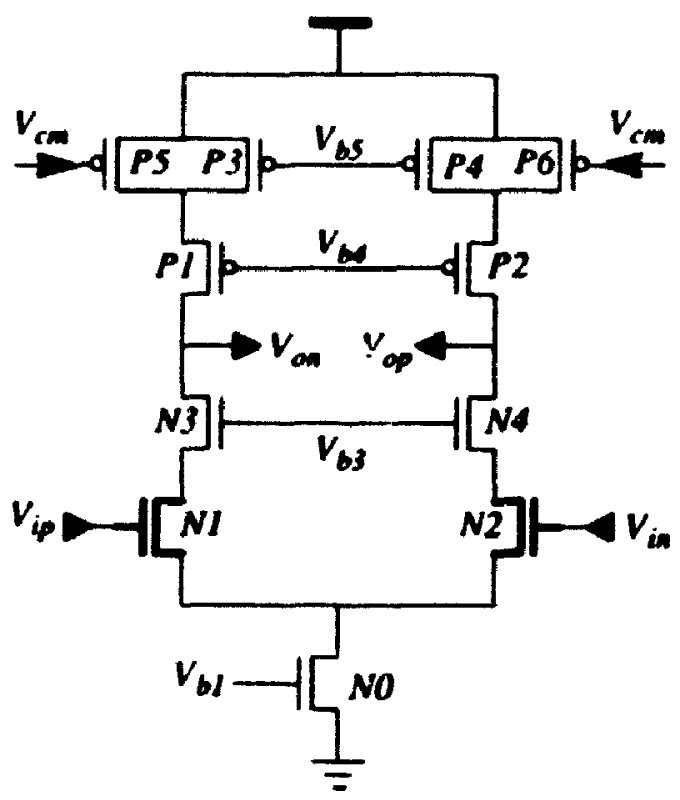

Figure 5.12: A fully differential cascode opamp

the output common mode voltage is at $3.5 \mathrm{~V}$. The output signal swing range is $\pm 0.5 \mathrm{~V}$ with respect to the output common-mode voltage. Simulated characteristics of the opamp 
designed in the $5 \mathrm{~V}$ option of the HCMOS5a process (0.5 $\mu \mathrm{m}$ CMOS from SGSthompson) are given in Table 5.3.

\begin{tabular}{|c|c|}
\hline Parameter & Value \\
\hline \hline DC gain & $61 \mathrm{~dB}$ \\
\hline Unity gain frequency & $360 \mathrm{MHz}$ \\
\hline Phase Margin & $63^{\circ}$ \\
\hline Slew rate & $500 \mathrm{~V} / \mu \mathrm{s}$ \\
\hline Differential Output swing & $2 \mathrm{~V}$ \\
\hline $\begin{array}{c}\text { Power dissipation } \\
\text { (not including CMFB cincuit) }\end{array}$ & $5 \mathrm{~mW}$ \\
\hline
\end{tabular}

Table 5.3: Simulated characteristics of opamp for IpF load capacitance

Power supply voltage was $5 \mathrm{~V}$ and the tail current was $1 \mathrm{~mA}$.

The design considerations for capacitor size and switch on-resistance are similar to those described in section 4.6 of the previous chapt:-r.

If low threshold natural MOSFETs are available in the process, they can be used as switches, pMOSFET capacitors, and as input differential pair transistors $(N 1$ and $N 2$ in Figure 5.12) to further optimize the circuit. A lower threshold voltage helps reduce the onresistance of switches, increases the linear region of the pMOSFET capacitor in strong inversion, and reduces the input common-mode level.

\subsubsection{Linear Charge Processor Viewpuint}

In section 2.5.3, it was shown that SC circuits are linear charge processors frum input to output. To a first-order approximation, linearity of SC circuits in the charge domain is independent of the non-linearity of the capacitors. However, since external signals are typically in the voltage domain, a linear input $V / Q$ and output $Q / V$ converter are required.

The output of a SC $\Sigma \Delta$ mudulator is a digital bit stream and as such no linear $Q / V$ converter is needed at the outfut. Input capacitors are the only critical components that 
must be linear.

A metal-metal capacitor seems to be a good choice for input capacitors. Misti:atch between a metal-metal capacitor and a MOSFET capacitor is expected to be large, however, that causes a gain error which is not important in a $\mathbf{\Sigma} \Delta$ modulator.

Our unity gain delay circuit is a special case of this charge domain principle where the circuit is also linear in the voltage domain.

\subsection{Summary}

In this chapter, non-linearity of MOSFET capacitors in a $0.5 \mu \mathrm{m}$ CMOS process was studied. It was sho wn that MOSFETs biased in the strong inversion regime have a better linearity than MOSFET capacitors biased in the accumulation regime because gate-poly depletion partially compensate the non-linearity in strong inversion. The voltage coefficients of a pMOSFET biased $2.5 \mathrm{~V}$ in strong inversion are measured to be $\alpha_{1}=42.7 \mathrm{kppm} /$ and $\alpha_{2}=7.7 \mathrm{kppm} / \mathrm{V}^{2}$, for a voltage swing of $\pm 0.5 \mathrm{~V}$. The impact of capacitor non-linearity on the performance of SC delay circuit was then analyzed. Finally, the design of a fourth-order bandpass sigma-delta modulator tolerant to MOSFET capacitors was presented along with Eldo simulation results. 


\section{Chapter 6}

\section{Conclusions and Future Work}

The rapid advance of CMOS technology into the deep submicron regime continues to improve the speed and packing density of integrated circuits. Device miniaturization, in deep submicron geometries, requires supply voltage down-scaling to ensure reliability. In the first part of this thesis, low-voltage circuits were considered in detail.

In deep submicron CMOS technologies, velocity saturation causes circuit speed improvement to slow down from $1.4 \mathrm{X}$ to about $1.2 \mathrm{X}$ every generation. Low voltage is also known to compromise circuit speed. The second pan of this thesis therefore focused on high-speed SC circuir techniques.

Low cost imfiementation of a mainly digital mixed-signal circuit requires analog circuits to be implemented in a standard digital CMOS process where double-poly is not available. In the last part of this thesis, linear SC circuit techniques in a digital CMOS process were investigated.

\subsection{Summary}

In Chapter 2, CMOS scaling in deep submicron was reviewed. In deep submicron technologies, the supply voltage is being scaled down to assure device reliability. In conjunction with the supply valtage, threshold voltage of MOSFETs is scaling down to attain circuit speed. Low voltage analog and digital circuit techniques were reviewed. For low-voltage analog circuits, SC and SI circuits were compared and it was shown that the SC technique has advantages over the SI technique from a signal-to-noise ratio point of view. 
Chapter 3 described low-voltage SC design techniques using low threshold voltage MOSFETs. Two methods for achieving low- $V$, MOSFETs in current CMOS processes were discussed. In the first scheme, natural threshold voltage MOSFETs fabricated in a dual poly gate CN.Jj processes (with $V,-200-300 \mathrm{mV}$ ) were proposed for lowvoltage mixed-signal circuits. The second method took advantage of short-channel effects and used short-channel MOSFETs as low-voltage analog switches.

Low threshold voltage MOSFETs are leaky and a detailed analysis of the effects of leaky switches on the accuracy of SC circuits was provided. Methods of reducing the off-current through MOSFET switches were described. These methods are (1) limiting the signal swing, (2) adjusting the $v$, by back bias, and (3) using novel low-leakage series transmission gate and composite switches.

Two experimental low-voltage SC sigma-delta modulators were presented. In the first design, an existing $3.3 \mathrm{~V}$ second-order $\Sigma \Delta$ modulator in a $0.8 \mu \mathrm{m}$ BiCMOS process was modified to operate at $2.25 \mathrm{~V}$. The modification involved reducing the channel length for all the switches from $0.8 \mu \mathrm{m}$ to $0.6 \mu \mathrm{m}$. The new design operates at a clock frequency of $2 \mathrm{MHz}$ and achieves a SNDR of $92 \mathrm{~dB}$ for an oversampling ratio of 256 . In the second design, a $1 \mathrm{~V}$ first-order SC sigma-delta modulator using low threshold voltage natural MOSFETs was reported. This modulator was tested at a clock frequency of $1 \mathrm{MHz}$. For an oversampling ratio of 128 the measured SNDR is about $54 \mathrm{~dB}$.

In Chapter 4 , high-speed $S C$ bandpass $\Sigma \Delta$ modulators were discussed. In the sampled-data domain, a direct implementation of resonators for bandpass sigma-delta modulator requires an efficient sample-and-hold circuit. A SC delay circuit was described and a novel double-sampled SC delay circuit was introduced. The impacts of circuit nonidealities on the performance of a simple SC delay cell and the double-sampled delay cell were analyzed. Two SC implementations for a fourth-order bandpass $\Sigma \Delta$ modulator were presented. The first design was a high-speed SC $\Sigma \Delta$ modulator based on a SC delay circuit and the second design was a double-sampled SC $\Sigma \Delta$ modulator, based on the doublesampled SC delay cell. Both designs were implemented in a $0.5 \mu \mathrm{m}$ CMOS process. The single-sampled modulator was tested at an $80 \mathrm{MHz}$ clock frequency. For an oversampling ratio of $\mathbf{4 0}$ the measured SNDR is about $42 \mathrm{~dB}$. The modulator consumes $50 \mathrm{~mW}$ from a 
3.3 V power supply. The double-sampled modulator was also tested at $80 \mathrm{MHz}$. The effective sampling frequency for this modulator is $160 \mathrm{MHz}$. Due to capacitor mismatch. the image signal was about $40 \mathrm{~dB}$ below the input signal. Measured SNDR is $30 \mathrm{~dB}$ (limited by the image) for an oversampling ratio of 16 (bandwidth of $5 \mathrm{MHz}$ ).

Chapter 5 explored the feasibility of using MOSFETs as linear capacitors in a SC circuit. It was shown that in a $0.5 \mu \mathrm{m}$ CMOS process. MOSFETs biased in strong inversion are more linear than MOSFETs biased in the accumulation regime. An analysis of distortion due to non-linearity of MOSFET capacitor in a SC delay cell was presented. Finally. design of a SC fourth-order bandpass sigma-delta modulator using pMOSFET capacitor was described.

\subsection{Conclusions}

Through analyses, simulations, and measurements of some experimental circuits, we demonstrated that mixed-signal linear SC circuits

- will scale down to at least $1 \mathrm{~V}$.

- can operate up to $160 \mathrm{MHz}$ in a $0.5 \mu \mathrm{m}$ CMOS using a double-sampled SC technique.

- are realizable in a standard digital CMOS process using MOSFET capacitors in strong inversion or accumulation.

\subsection{Future Work}

High-performance SC design from the perspective of this thesis, namely at low voltages, high speeds, and implemented in a digital CMOS process, needs further development. Some promising areas of research are as follows:

Low-Volt : SC

In this thesis, we showed that low threshold voltage MOSFETs are suitable for I V mixed-signal application. The next step is to integrate a second-order sigma-delta modulator with the decimation filter in order to demonstrate high resolution at I V and see if noise coupling from digital circuit will degrade the SNR performance. 
Chapter 6 . Conclusions and Future Work

150

High -Speed SC

A major limitation of a double-sampled SC circuit is image problems caused by mismatch between the two channels as well as non-uniform sampling. Architectures that are immune to mismatch would be an interesting area for research. In a $\mathbf{\Sigma} \boldsymbol{\Delta}$ modulator, the effect of channel mismatch might be compensated for by an LMS algorithm in DSP.

Linear SC in a Digital CMOS Process

In this dissertation, it was shown that poly-depletion causes MOSFETs to behave more linearly in strong inversion than in accumulation regime. Further investigation and implementation of some SC test circuits are required to verify this property.

In summary, analog SC circuit techniques remain viable and vital in the design of highperformance mixed-signal circuits in deep submicron CMOS technologies. 


\section{Appendix A}

\section{MOSFET Equations}

The $I_{D S}-V_{D S}$ characteristic of MOSFET in strong inversion $\left(v_{(i S}>V_{1}\right)$ can be approximated by an $\alpha$-power law [Sakurai9()] as follows:

$$
i_{D S}=\beta\left(v_{G S}-V_{1}\right) \alpha
$$

$V_{t}$ is the threshold voltage of the MOSFET, $\alpha$ is the velocity saturation index which is a number less than 2 , and $\beta$ is:

$$
\beta=\frac{1}{2} \mu C_{o x} \frac{W}{L}
$$

In this equation, $\mu$ is the carrier mobility, $C_{o x}$ is the gate oxide capacitance, $W$ is the MOSFET channel width, and $L$ is the MOSFET channel length.

The delay time of an inverter (using results in [Burns64]) is then approximately

$$
\tau_{D}=\frac{C_{L} V_{D D}}{\beta\left(V_{D D}-V_{t}\right)^{\alpha}} .
$$

where $C_{L}$ is the load capacitance.

For $v_{G S}<V_{1}$, nMOSFETs operate in weak inversion (or subthreshold regime) with an exponential $i_{D S}-v_{G S}$ relationship as follows:

$$
i_{D S}=I_{D 0} \frac{W}{L} e^{\frac{v_{C S}-V_{1}}{n \phi_{1}}}\left(1-e^{-\frac{v_{D S}}{\phi_{1}}}\right)
$$


Here, $\phi_{l}$ is the thermal voltage and $I_{D 0}$ the MOSFET specific current (the value of the drain current for unit transistor $(W / L=1)$ with gate to source voltage biased at threshold voltage), and $n$ is the subthreshold slope factor. The equations for thermal voltage, the specific current, and subthreshold slope factor are:

$$
\begin{gathered}
\phi_{1}=\frac{k T}{q} \\
I_{D 0}=\mu C_{o x} \phi_{l}^{2} \\
n=1+\frac{C_{D}}{C_{o x}}
\end{gathered}
$$

Here. $k$ is the Boltzman constant, $T$ is temperature in degree Kelvin, and $C_{D}$ is the depletion layer capacitance. 


\section{Appendix B}

\section{Natural MOSFET Characteristics}

Low- $V$, natural threshold voltage transistors can be fabricated as an option in a dual poly gate CMOS process. A process designed for IV operation may be further simplitied by eliminating the steps required for hot-carrier reduction i.e., LDD (Lightly doped Drain) implant and formation.

Natural threshold voltage MOSFETs were fabricated in a $0.5 \mu \mathrm{m}$ CMOS process by removing the threshold adjust masks and implants. Figure B.1 and Figure B.2 show the measured $I_{D}$ and $G_{m}$ versus gate voltage for a $20 \mu \mathrm{m} / 0.5 \mu \mathrm{m}$ nMOSFET and a $20 \mu \mathrm{m} / 0.5 \mu \mathrm{m}$ pMOSFET biased at $\left|v_{D S}\right|=0.1 \mathrm{~V}$ respectively. For $0.5 \mu \mathrm{m}$ channel length, the threshold voltages are measured to be $202 \mathrm{mV}$ and $197 \mathrm{mV}$ for $\mathrm{nMOSFET}$ and pMOSFET respectively and subthreshold slopes are about $80 \mathrm{mV} / \mathrm{dec}$ ade .

Measured $\mathrm{I}_{D S}-\mathrm{V}_{\mathrm{DS}}$ characteristics for natural threshold nMOSFET and pMOSFET are illustrated in Figure B.3 and Figure B.4 respectively. In this technology, $\alpha$ for an nMOSFET is 1.27 and for a pMOSFET is 1.32 . 


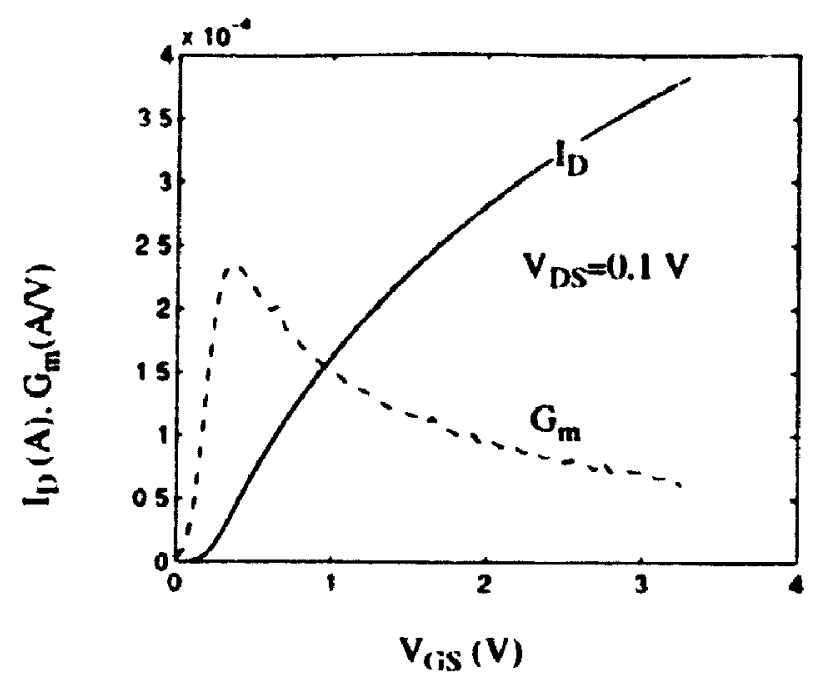

Figure B.1: Measured $I_{D}(A)$ and $G_{m}(A / V)$ versus $V_{G S}(V)$ for "natural" nMOSFET

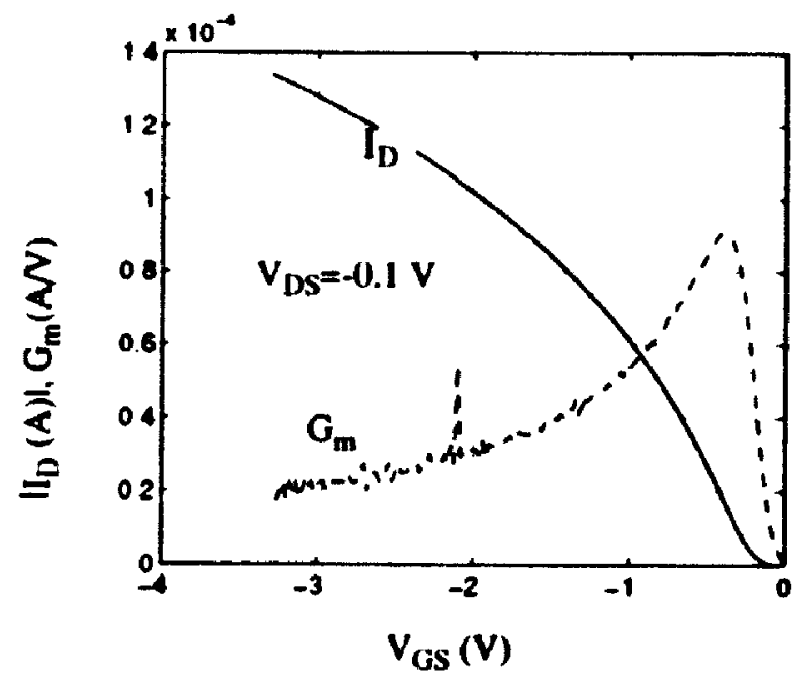

Figure B.2: Measured $l_{D}(A) l$ and $G_{m}(A / V)$ versus $V_{G s}(V)$ for "natural" pMOSFET 


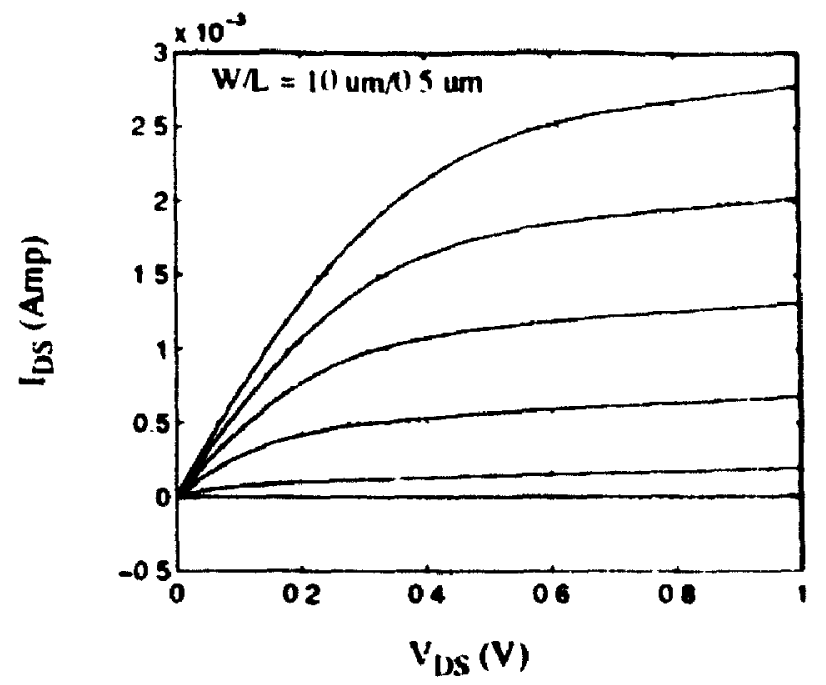

Figure B.3: Me:sured nMOSFET $I_{D S}-V_{D S}$ characteristics. $I_{D S} \sim\left(V_{G S}-V_{1}\right)^{1.27}$

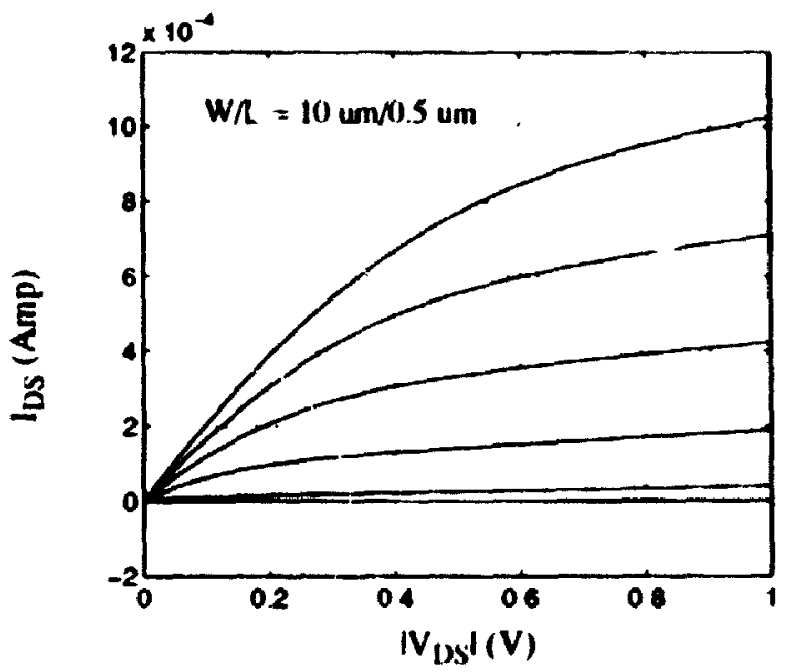

Figure B.4: Measured pMOSFET $I_{D S}-V_{D S}$ characteristics. $I_{D S} \sim\left(V_{G S}-V_{1}\right)^{1.12}$ 


\section{References}

[AdachiY)] Toshio Adachi, A. Ishikawa. A. Barlow, and K. Takasuka, "A 1.4V Switched Capacitor Filter," in Proc. CICC. 1990, pp. 8.2.1-8.2.4.

[Au46] S. Au and B. Leung," A 1.95V. 0.34mW 12-bit Sigma-Delta Modulator Stabilized by Loxal Feedback Loops," in Proc. CICC. 1996. pp. $411-414$.

[Battershy91] N.C. Battersby and C. Toumazow. "A new generation of class AB switched-current memory for analog sampled-data applications," in Proc. Incernational Symposium on Circuits and Systems. June 1991, pp. 2569-2572.

[Battershyy.3] Nicholas C. Battersby. Switched-Current Techniques for Analog Sampled-Data Signul Processing. Ph.D. Thesis. Imperial College (University of London), Feb. 1993.

[Bivarjaniy5a) Seyfi Bazarjani. Martin Snelgrove, and Tom MacElwee. “A IV Switched-Capacitor ES Modulator." in Proc IEEE Symposium on Low Power Electronics. San Jose. CA. pp. 70-71. Octoher 1995.

[Bazarjani95b] Seyti S. Bazarjani. Tom MacElwee. and Martin Snelgrove. "Optinizing the Natural MOSFETs in a 0.5um Dual Poly Gate CMOS Process for IV Mixed-signal Applications." in Proc. 25ih European Solid State Device Research Conference. pp. 675-678. The Hague. The Netherlands. September 1995.

[Bazarjani95c] Seyfi Bazarjani and Martin Snelgrove. "A 4th Order SC Bandpass Es Modulator Designed on a Digital CMOS Process," in Proc. 38th Midwest Symposium on Circuits und Sysiems. pp. 1345-1348, Rio de Janeiro, Brazil, August 1995.

[Bararjani95d] Seyfi S. Bararjani and Martin W. Snelgmve. "Low Voltage SC Cincuit Design with Low-V, MOSFETs," in Proc. International Symposium on Circuits and Systems. pp. 1021-1024. Seatte. May 1995.

[Bazarjani94a] Seyfi Bazarjani, Martin Snelgrove, Garry Tarr, and Kathy Howlet, "Low Voltage SC Cincuit Design Using Shon-Channel MOSFET Switches," in Proc. Ist Internarional Conference on AS/C. Beijing. Chinin pp. 344-347, October 1994.

[Bivargani44b] Seyfi Bazarjani and Martin Snelgrove, "Single-Battery Mixed Analog/Digital Signal Processing with Natural Transistors." in Proc. 1994 IEEE Internatic. "al Workshop on Low Power Design. Napa. CA. pp. 219-224. April 1994.

[Bazarjani93] Seyf Bazarjani, Switched Current Analog Circuit Design. Technical repon. Carleton University, April 1993.

[Behry2] A.T. Behr. M.C. Schneider, S.N. Filho, and C.G. Montoro, "Harmonic Distontion 
Caused by Capacitors Implemented with MOSFET Gates." IEEE Journul of Sollid-Sicure Circutrs. vol. 27. no. 10. pp. 1471-1475, Oct. 1992.

[Bermude292] J.C.M. Bermudez. M.C. Schneider. C.G. Mumtoro, "Compatihility of switched tapacitur fikers with VLSI processes." IEE proc. Circuits Devices Sust.. vol. 1.39. mu. 4. Ip.+113. 418. Aug. 1992.

[Boothroydy2] A.R. Bochroyd. et al. "MISNAN-A Physically hased continumus MOSFET imukel tww CAD applications." IEEE Trans on computer-ulded design. voll. III. Mp. 1512-1524. Dec. 1991.

[Bosier88] B. Boser and B. Wooley. "The design of sigmandelta modulation anadog to digutid conventers." IEEE Journal of Solid-Siate Cirimits. vol. 23. pp. I 24\%-1 HKK, Dec. IYKK.

[Brews80] J.R. Brews, et al.. "Generalized guide for MOSFET miniaturizatium." ItEE Eler"lon Device Letters. vol. EDL-1. pp. 2-3, Jan. 1980.

[Burmas95] T. Bumas. S. Lewis. P. Hurst. and K. Dyer." A Second-Order Dinhle-Sanpled LeltaSigma Modulator." in Proc. CICC. 1945. pp. 195-198.

[Bums64] J. R. Bums. "Switching response of complementary symunetry MOS transisturs kngu" circunts." RCA review, pp. 627-661, Dec. 1964.

[Bur91] James B. Burt and Allen M. Peterson. "Energy Consideration in Multi-Mindule hasiod Multiprocessors." in Proc. IEEE InI. Conf, on Computer Design. 1941 . pp. 59 1.t(1).

[Burr94] James B. Burr and John Scon." A 200mV Self-Testing Encoder/Decruder using Staulind Ultra-Low-Power CMOS." in ISSCC Digest of Technical Pupers. Feb. 1944. Im. K4 - 55.

[Burr95]

[Callias89]

James B. Burr. "Cryogenic Ulura Low Power CMOS." in Pror. IEEE Simposium an Low Power Electronics95. San Jose. CA. pp. 82-83. Oct. 1495.

F. Callias, F.H. Salchli, and D. Girard, "A set of four ICs in CMOS technokgny tor programmable hearing aid." IEEE Jowrral of Solid-State Circuts. vol. SC-20, mo. 2. In. 301-312, April 1989.

[Candy92] J.C. Candy and G. C. Temes. "Oversampling Methods for AD and D/A Conversikn." "n Oversampling Delia-Sigma Converiers. Theory and Simulatwns. IEEE Press I $\$ 42$.

[Castello85] Rinaldo Castello and Paul R. Gray. "Performance Limitations in Swikched-Capaciut Filters," IEEE Tran. Circuits and Syst.. Vol. CAS-32. pp.865-876. September. 14\%5.

[Castelloy)] Rinaldo Castello and Luciano Tomasini, "1.5-V High-Performance SC Filters un BiCMOS Technology." IEEE Journal of Solid-State Cirruits. Visl. 26. mo. 7. pp. $43($ ). 936. July 1991. 
[Chany9] C. Chan. H. Ling, and O. Choy, "A One Voll Four-Quadrant Analog Current Mode Multiplier Cell." IEEE Journal of Solid-State Circuits. vol. 30. no. 9. pp. 1018-1019. Sep. 1995.

[Chandrakasany2] A. Chandrankasan. S. Sheng, and R.W. Brodersen. "Low-Power CMOS Digital Design." IEEE Journal of Solid-Slate Circuirs, vol. 27. no. 4. pp. 473-483. April 1942.

[ChenY)] Juhn Y. Chen. CMOS Devices and Technalogy for VLSI Englewood Ciffs, NJ: Prentice-Hall. Inc.. 1990.

|Choi(x)|

T.C. Choi and R.W. Broderson, "Considerations for High-Frequency SwitchodCapacitor Ladder Filters." IEEE Transactions on Circuits and Systems, vol. CAS-27. mo. 6. pp. 545-552. June 1980.

[Crawleyy2) P. Crawley and G. Roberts. "A component-Invariant Second-Order Switched-Curreat Sigma-Delta Modulator," in Proc. Invernational Symposium on Circuits and Systems. 1942. pp. 1324-1327.

[Crols44] J. Crols and M. Steyaen. "Switched-opamp: An approach to realize full CMOS switched-capacitur cincuits at very low power supply voltages." IEEE Journal of SolidStute Circuits, vol. 29. pp. 936-942. Aug. 1994.

[Davariy5] Bijan Davari. R. H. Dennard. and G. G. Shahidi. "CMOS Scaling for High Performance and Low Power-The Nexi Ten Years," in Proc. IEEE. vol.83. pp.595-606. Ap. . 1995.

IDennard74] R.H. Dennard. F.H. Gaensslen. H.N. Yu. V.L. Rideout. E. Bassous, and A.R. LeBlanc. "Design of ion-implaned MOSFET's with very small physical dimensions." IEEE Jowrnat of Solid-State Circuts. vo. SC-9. pp. 256-268. May 1974.

|Eldo| ELDO User's Manual. ANACAD Electrical Engineering Software. 1995.

[Fier(x)]

[Gehm42]

T. Fiez and D.J. Allstor. "CMOS Switched-Current Ladder Filters." IEEE Journal of Sulid-Stote Circuits. vol. SC-25. pp. 1360-1367. Dec. 1990.

M. Gehm. P. Jaenen. V. Van Diessche. A. Goethals. N. Samarakone, L. Van den Hove. and B. Denturck, "Evaluation of Methods to Reduce Linewidh Variation due to Topography for I-line and Deep UV Lithography." in Proc. SPIE. vol. 1674. pp. 681700. 1992.

[Gray93] PR. Gray and R.G. Meyer, Analysis and Design of Amalog Incegrated Circuits, 3rd ed. New Yar: John Wiley \& Sons, 1993.

[Gregorian\&1] R. Gregorian. "High-resolution switched-capacitor D/A convener." Microelectronics J. . pp. 10-13. no. 12. 1981. 
[Gregorian 86]

[Grilu\%6]

[Hivawayy]]

[Haug84]

[Hairaper46]

[Horowitz'44]

[Howletty3]

[Hu94]

[Hughes89]

[Hughes90]

[Hughes 90 a]

[Hughes92]

[Hughes43]

[Huijsing85] 1986. John Wiley \& Sons.

R. Gregorian. and G.C. Temes, Analog MOS Invegrated Circuits for Sigmal Prucessing.

J. Grilo. E. Mar Robhic. R. Halion. and Gahor Temes, "A 1.8V 44dB Dynimix Range

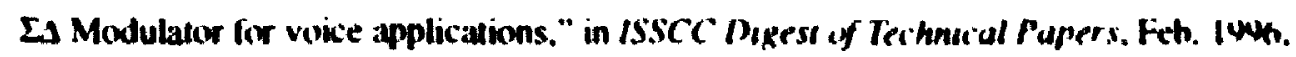
pp. $2312-231$.

R. Hadaway. et al., "A Sub-Micron BiCMOS tachuology fir Teleconnmunicatinn." un Proc. of 21 si European Solid Stule Device Researih Conference. Mp. S13-51n. Montreux. Sept. 1991.

K. Haug. G.C. Temes and K. Martin. "Improved offset compensation sithemes tor SC cincuits." in Proc. International Simposium on Circuits and Sistems. Pp. IIS4-1057. 1984.

A. Hairapetian. "An 8IMHz IF Receiver in CMOS." in IS.SCC Dige'st "f leinnuiul Pupers, Feb. 1946, San Francisco, CA, pp. 56-57.

M. Horowitz. et al. "Lon-power digital design." in Proc. ItEE Svmpnoseum in Lin. Power Electronics. San Diego. CA. pp. 8-11. Oct. 1944.

K. Howlett. Nonel Semiconductors, private communicunten, 149?.

C. Hu. "Low-Voltage CMOS Device Scaling." in ISSCC Degest of Jechnecul Papiers. Feb. 1994. San Francisco. CA. pp. 86-87.

J.B. Hughes. N. Pind. and I.C. Marbeth. "Switched-Current - A New Technique fir Analog Sampled-Daka Signal Processing." in Prox IEEE Internauomul Svmpouseum un Circuits and Systems, pp. 1584-1587. 1989.

J.B. Hughes. N. Bird. and I.C. Macbeth. "Second Generation Switched-Current Signtal Processing." in Proc. IEEE International Symposium on Circus and Sivstems. Ip. 2805-2808. 1990.

J.B. Hughes. I.C. Macbeth. and D.int. Patullo. "Switched current tilters." in ItI: I..' G. Electron Circuts a Syst., April !4xo. pp. 156-162.

J.B. Hughes, et al."Switchod-Cirrent Video Signal Prucessing." in Prou CICC. 1442. pp. 24.4.1-24.4.4.

J.B. Hughes and K. Moulding. " $s^{2} I$ : A Switchod-Current Techunque for High Performance," Electronics Letters, vol. 24, pp.1560-1662. Dec 1441.

J.H. Huijsing and D. Lincharger. "Low-voltage operatuonal anopliticr with raul-10-raul input and output ranges." IEEE Journal of Solut-Siale Circuts. vol. SC:20, ,p. 1144. 
1150. Dec. 1985.

[Htirsty)] PJ. Hurst and W.J. McIntyre, "Double Sampling in Switched-Capacitor Delta-Sigma AV D Converter." in Proc. IEEE International Symposium on Circuits and Systems, 1990. pp. 902-905.

[Hursry2] P.J. Hurst and K.C. Dyre, "An Improved Double Sampling Scheme for SwitchedCapacitor Delta-Sigma Modulators," in Proc. IEEE International Symposium on Circuits and Systems. 1992, pp. 1179-1182.

[Jantzi]

S. Jantzi. R. Schreier and M. Snelgrove. "The Design of Bandpass Delta-Sigma ADCs." chapter in Delta-Sigma Data Converters. IEEE Press, to appear.

|jantzi921 S. Jantzi, M. Snelgrove. and P.E. Ferguson Jr., "A 4th-order bandpass sigma-delta modulator," in Proc. CICC. May 1992. pp. 16.5.1-16.5.4.

[Kakumu( $($ )] $\quad$ M. Kakumu and M. Kinugawa.. "Choice of Power-Supply Voltage for Half-Micrometer and Lower Submicrometer CMOS Devices," IEEE Trans. Electron Devices, vol. 37, no. 5. pp. 1334-1342, May 1990.

[Keyes75] R. Keyes, Appl Phys 8. p. 251. 1975.

[Kobayashi94] T. Kobayashi, and T. Sakurai, "Self-Adjusting Threshold-Voltage Scheme (SATA) for Low-Voltage High-Speed Operation," in Proc. CICC. May 1994, pp. 271-274.

[Krurnmen82] F. Krummenacher. “Micropower Switched Capacitor biquadratic cell," IEEE Journal of Solid-State Circuits, vol. SC-17. pp. 507-512. June 1982.

[Krumınenx3] F. Krummenacher, H. Pinier, and A. Guillaume, "High sampling rates in SC circuits by on chip clock-voltage multiplication." in Proc. ESSCIRC '83. Lausanne. Switzerland. pp. 123-126. Sep. 1983.

[Kuroda95] T. Kuroda, and T. Sakurai. "' Tverview of Low-Power ULSI Circuit Techniques," IEICE Trans. on Electron. vol. E-78-C. no. 4, pp. 334-342, April 1995.

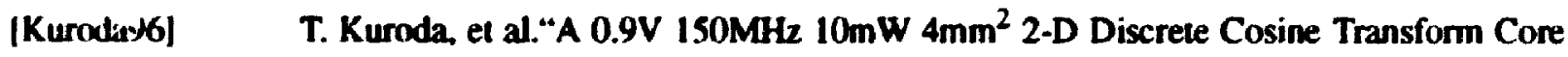
Processor with Variable-Threshold-Voltage Scheme," in ISSCC Digest of Technical Papers. Feb. 96. pp. 166-167.

[Lee85] K.L. Lee and R.G. Meyer. "Low-disurtion switched-capacitor filter design techniques," IEEE Journal of Solid-Staie Circuits, vol. Sc-20, pp. 1103-1113, 1985.

[Leuenhergerty] T. Leuenberger and E. Vittoz, "Complementary-MOS low-power low-voltage integrated binary counter." Proc. IEEE. vol. 59. pp. 1528-1532. Sep. 1969.

[Liu93] D. Liu and C. Svensson. "-Trading speed for low power by choice of supply and 
[Longo93] L. Longo, B. Homg. "A 15b 30kHz Bandpass Sigma-Delta Modulator," in ISSCC Digest of Technical Papers. Feb. 93. San Francisco, CA. pp. 226-227.

[Martin87] K. Martin. L. Ozcolak. Y.S. Lee, and G.C. Temes, “A Differential Switched-Capacitor Amplifier." IEEE Journal of Solid-State Circuits. vol. SC-22. pp. I(14-I(K. Feb. 1487.

[Matlab] MATLAB Reference (juide. The MarhWorks Inc., 1994.

[Matsuya94] Yasuyuki Matsuya and 1. Yamada, "IV Power Supply, Low-Power Consumption AD Conversion Technique with Swing-Suppression Noise Shaping." IEEE Journal of SolidState Circuits, vol. 29. pp.1524-1530. Dec. 1994.

[Mizuno93] T. Mizuno et al.. Symp. on VLSI Tech. Digest. p.41. 1993.

[Mizuno94] T. Mizuno et al., "Performance Fluctuation of a 0.1 $\mu \mathrm{m}$ MOSFETs-Limitition of $11.1 \mu \mathrm{m}$ ULSIs," Symp. on VLSI Technology Digest, pp.13-14. 1994.

[Montoro88] C.G. Montoro and J.C.M. Bermudez, "Switched capacitor circuits fully compatihle with digital Si-gate single poly technology," in Proc. 31 st Mudwest Symposium on Cirruts and Systems, St. Louis, MO, pp.1-3, August 1988.

[Mutoh93] S. Mutoh, T. Douseki, Y. Matsuya, J. Yamada and T. Aoki, "IV high speed digital circuit technology with $0.5 \mu \mathrm{m}$ multi-threshold CMOS," in Pruc. IEEE Int. AS/C ronf.. pp. 186-189, Sep. 1993.

[Nagane69] K. Nagane and T. Frank. " $\mathrm{Al}_{2} \mathrm{O}_{3}$ complementary MOS transistors," in Prox. I.st Conf Solid-State Devices. pp. 132-136. Tokyo. 1969.

[Nedved95] J. Nedved, J. Vanneuville, D. Gevaen, and J. Sevenhans, "A Transistor-Only Swikched Current Sigma-Delta AD Conventer for CMOS Speech CODEC." IEEE Journal of Solid-State Circuits, vol. 30, no. 7. pp. 819-822, July 1995.

[Nishinohra92] K. Nishinohara et al. IEEE Tuns Elect Dev. ED-39 p.634 1992.

[Norman96] O. Norman. “A Band-pass Is Modulator for Uttrasound Imaging at 160MH. ('lexk Rate," in ISSCC Digest of Technical Papers. Feb. 1996, San Francisco. CA. pp. 236237.

[Ribner91] D.B. Ribner. "Double rate oversampled interpolative modulaturs for analog-(1)-digital conversion," 1991 US Patent NO. 5.030,954 (filed 1999)).

[Sackinger87] E. Sackinger, and W. Guggenbuhl, "A versarile building blocks for CMOS differental arr.plifier," IEEE Journal of Solid-Siale Circuits, vol. SC-42. no. 2. pp.287-294, 1987.

[Sakurai90] T. Sakurai, and A.R. Newton. "Alpha-Power Law MOSFET Model and its Applicaluons 
to CMOS Inverter Delay and Other Formulas," IEEE Journal of Solid-State Circuits. vol. 25, no. 2, pp.584.594. Apr. 1990.

[Sano88]

E. Sano, T. Tsukahara, and A. Iwata "Perlormance Limits of Mixed Analog/Digital Circuits with Scaled MOSFET's," IEEE Journal of Solid-State Circuits, vol. 23, no. 4. pp.942-949, Aug. 1988.

[Schneider94] M.C. Schneider. C.G. Montoro, J.C.M. Bermudez, "Explicit formula for harmonic distortion in SC filters with weakly nonlinear capacitors." IEE proc. Circuits Devices Syst., vol. 141, no. 6. pp.505-509, Dec. 1994

[Schutz93] Joseph Schutz. "Low Power Design Techniques for Microprocessors." 1993 IEDM. Shon Course Program.

[Senderow94] D. Senderowicz, "CMOS Operational Amplifiers," in Design of MOS VLSI circuits for relecommunications, edited by J. Franca and Y. Tsividis. Prentice-Hall. 1994.

[Shoaei94] Omid Shoaei and Martin Snelgrove, "Optimal (Bandpass) Continuous-Time $\Sigma \Delta$ Modulator," in Proc. IEEE International Symposium on Circuits and Systems. May 1994. pp. 489-492.

[Shinichiro93] M. Shinichiro, T. Douseki, Y. Matsuya, T.Aoki, and J. Yamada “IV High-Speed Digital Circuit Technology with 0.5um Multi-threshold CMOS," in Froc. CICC, May 1993. Pp. 186-189.

[Shoji92]

M. Shoji, Theory of CMOS Digital Circuits and Circuit Failures, Princeton N.J.. U.P. 1992.

[S1A94]

National Technology Roadimap for Semiconductors, Semiconductor Indusury Association. 1994.

[Singor94] ～Frank W. Singor. W.M. Snelgrove. "10.7MHz Bandpass Delta-Sigma AD Modulators." in Proc. CICC. May 1994. pp.163-166.

[Song95]

B. Song. “A 4th-Order Bandpass DS Modulator with Reduced Number of Opamps," in ISSCC Digest of Technical Papers, Feb. 1995, pp. 204-205.

[Soreefan96] R. Soreefan. M.A.Sc. Thesis in preparation. Carleton University.

[Stein77] K. Stein, "Noise-Induced Error Rate as Limiting Factor for Energy per Operation in Digital IC's," IEEE Journal of Solid-State Circuits, vol. sc-12, no. 5, pp. 527-530, Oct. 1977.

[Sung2]

Shih. W. Sun, et al, "A fully complementary BiCMOS technology for sub-halfmicrometer microprocessor applications." IEEE Trans. Eleciron Devices. vol. 39, pp. 
2733-2738, Dec. 92.

[Swanson72] R.M. Swanson, and J.D. Meindl. "Ion-Implanted Complementary MOS Transisiors in Low-Voltuge Circuits," IEEE Journal of Solid-State Circuits, vol. sc-7, no. 2. Mp. 146153. Apr. 1972.

[Swanson74]

[Tan95]

[Tanimoto91]

R.M. Swanson, "Complementary MOS Transistors in micropower (incuits." Ph.l) Thesis. Stanford University. December 1974.

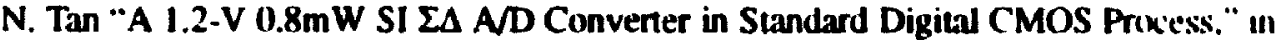
Proc. ESSCIRC`95, Lille, France, pp. 150-153, Sep. 1995.

H. Tanimoto, M. Koyama and Y. Yoshida, "Realization of a I-V Active Filter Using Linearization Technique Employing Plurality of Emitter-Coupled Pairs." IEtE Jour nul of Solid-State Circuits, vol. 26. no. 7. pp. 937-944. July 1991.

[Tarr95] G. Tarr. "Compact Device Model Parameters for Low-power $11.5 \mu \mathrm{m}$ and $10.35 \mu \mathrm{m}$ CMOS." An internal Nortel technical report. 199.5.

[Temes93] $\quad$ G. Temes, P. Deval, and V. Valencic, "SC Circuits: The State of the An Compared w St Techniques." in Proc. IEEE International Symposium on Circuits and Svsiems. 1993. pp. $1231-1234$.

[Thomas93] Jim Thomas. "Low Power For Communications and Control," I99.1 IE1DM, Shun Course Program.

[Toumazou90] C. Toumazou. J.B. Hughes. and D.M. Pattullo. "Regulated Cascode Switched-Curren Memory Cell." Electronics Letters, vol. 26. no. 5, pp. 303-305, March (991).

[Troutman79] R.T. Troutman, “VLSI Limitations from Drain-Induced Barrier Lowering," IEEE Trans Electron Devices, vol. ED-26, pp. 461 468, April 1979.

[Tsividis87] Y.P. Tsividis, Operation and Modeling of the MOS Transistors. McGraw-Hill Buok Company, New York, 1987.

[Varelas96] T. Varelas, S. Bazarjani, and M. Snelgrove, "A Bipolar Sampled-Dala Barkdpass DeltaSigma AVD Modulator." in Proc. CICC, May 1996, pp. 205-208.

[Vittoz77] E. Vittoz and J. Fellrath. "CMOS analog integrated circuits based on weak inversion or ation," IEEE Journal of Solid-State Circuits, vol. sc-12, pp. 224-231, June 1977.

[Vittoz80] E. Vittoz and F. Krummenacher, "Aicropower SC Filters in Si-gate Technology," in Proc. ECCTD '80, Warsaw, pp.61-72, 1980.

[Vittoz93] E. Vittoz. "Micropower Techniques," in J. Franca and Y.Tsividis, Eds., Design of Analog-Digisal VLSI Circuits for Telecommunications and Signal Processing. Prentice 
Haall. 1993.

[Vitu,194] E. Vittoz. "Low-Power Design: Ways to Approach the Limits," in ISSCC Digest of Technical Papers, Feb. 1994. pp. 14-18.

[Wayney2] D. Wayne, et al., "A single-chip hearing aid with one volt switched capacitor filters," in Proc of IEEE 1992 CICC. pp. 7.5.1-7.5.4. 1992.

[Wegmann87] G. Wegmann. E.A. Vittoz. and F. Rahali, "Charge Injection in Analog MOS Switches," IEEE Journal of Solid-State Circuits. vol. sc-22, no. 6. pp. 1091-1097. Dec. 1987.

[Widlar78] R. Widlar, "Low-Voltage Techniques," IEEE Journal of Solid-State Circuits, vol. sc-3. no. 6, pp. 838-846, Dec. 1978.

[Wong83] S. Wong and C.A.T. Salama, "Impact of scaling on MOS analog performances," IEEE Journal of Solid-State Circuits, vol. SC-18. no. 1. pp. 106-114. Feb. 1983.

[Wong93] H.S. Wong and Yuan Taur, "Three dimensional "Atomistic" Simulation of Discrete

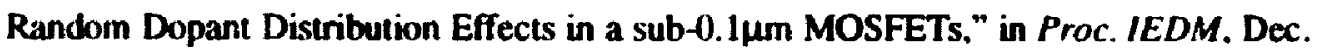
1993. pp. 705-708.

[Y:ung94] H.K. Yang and E.I. El-Masry, "A novel double sampling cechnique for delta-sigma modulators." in Proc. 37th Midwest Symposium on Circuits and Systems, Lafeyette, Louisiana. August 1994.

[Yoshizawa95] H. Yoshizawa and G.C. Temes. "High-Linearity Switched-Capacitor Circuits in Digital CMOS Technology," in Proc. International Symposium on Circuits and Systems. pp. 1029-1032, Seatte, May 1995.

[Yoshizawa96] H. Yoshizawa, G.C. Temes, P. Ferguson Jr., and F. Krummenacher. "Novel Design Techniques for High-Linearity MOSFET-Only Switched-Capacitor Circuits," in Proc. VLSI Symposium, 1996. 

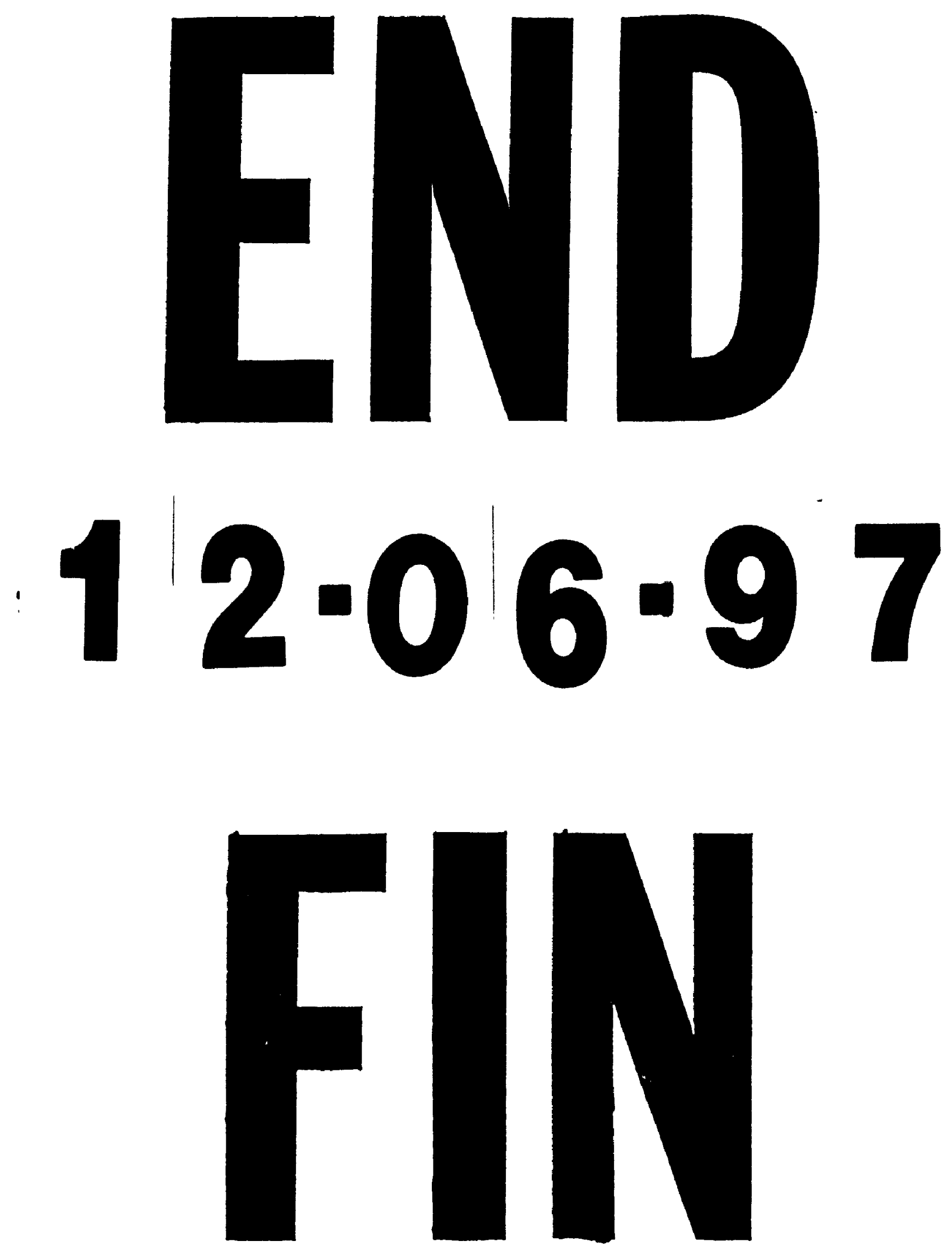